Supporting Information for

\title{
Chemistry of 2,14-Dithiacalix[4]arene: Searching for the Missing Fifth Conformer
}

Daniel Kortus, ${ }^{\dagger}$ Ondřej Kundrát, ${ }^{\dagger}$ Jan Čejka, ${ }^{\S}$ Hana Dvořáková ${ }^{\ddagger}$ and Pavel Lhoták ${ }^{\dagger *}$

'Department of Organic Chemistry, University of Chemistry and Technology, Prague (UCTP),

Technická 5, 16628 Prague 6, Czech Republic

${ }^{\ddagger}$ Department of Solid State Chemistry, UCTP, 16628 Prague 6, Czech Republic.

${ }^{\S}$ Laboratory of NMR spectroscopy, UCTP, 16628 Prague 6, Czech Republic.

\section{Table of contents}

1. Spectral characterisation

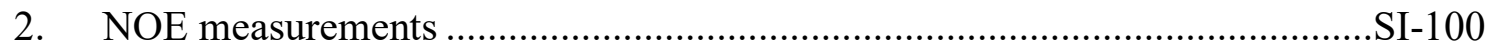

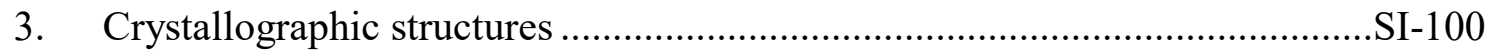

4. Results of quantum chemical calculations ...................................................SI-113 


\section{SPECTRAL CHARACTERISATION}

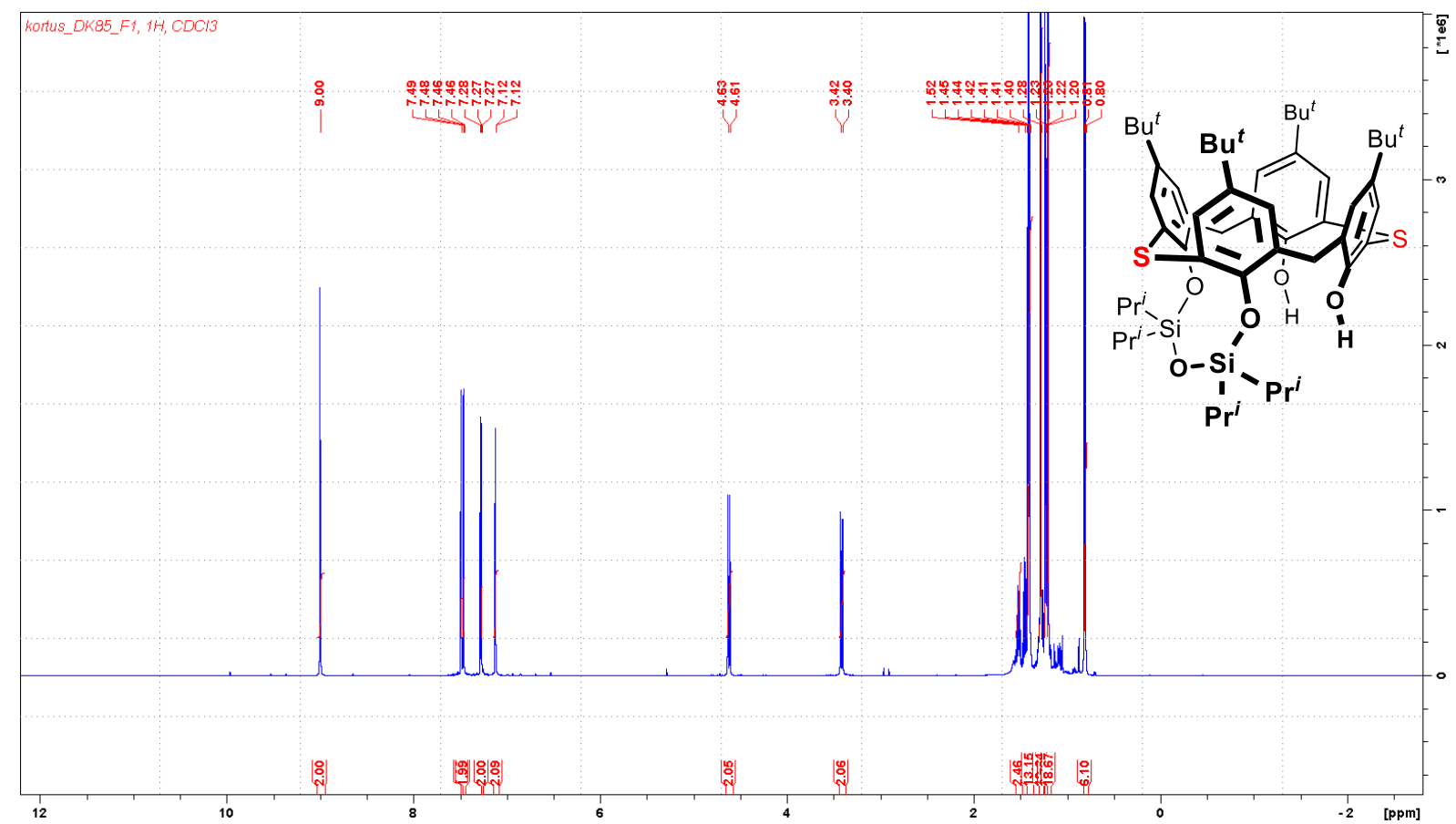

Figure S1. ${ }^{1} \mathrm{H}$ NMR of compound $2\left(\mathrm{CD}_{2} \mathrm{Cl}_{2}, 600 \mathrm{MHz}\right)$.

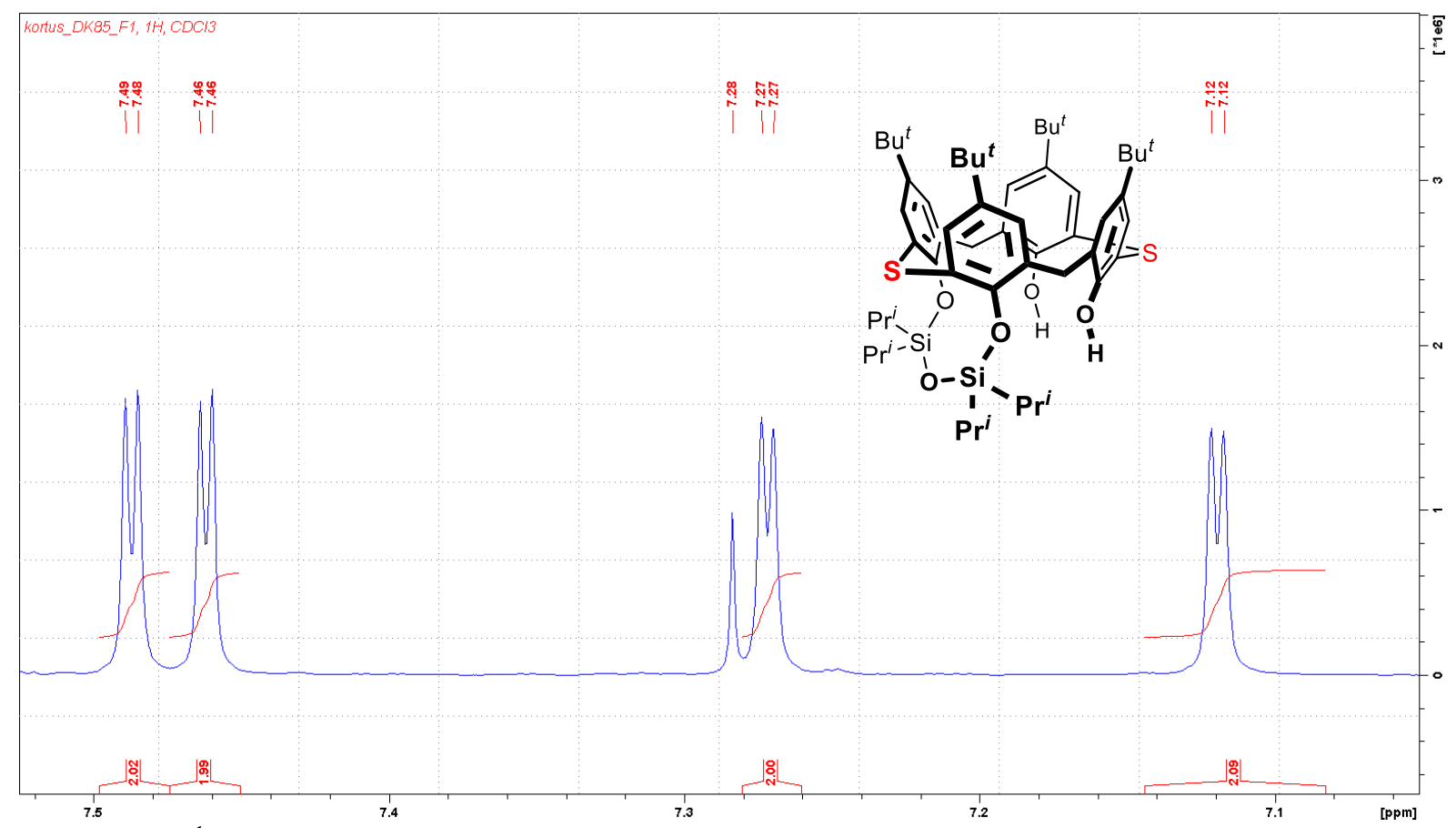

Figure S2. ${ }^{1} \mathrm{H} \mathrm{NMR}$ of compound 2, aromatic region $\left(\mathrm{CD}_{2} \mathrm{Cl}_{2}, 600 \mathrm{MHz}\right)$. 


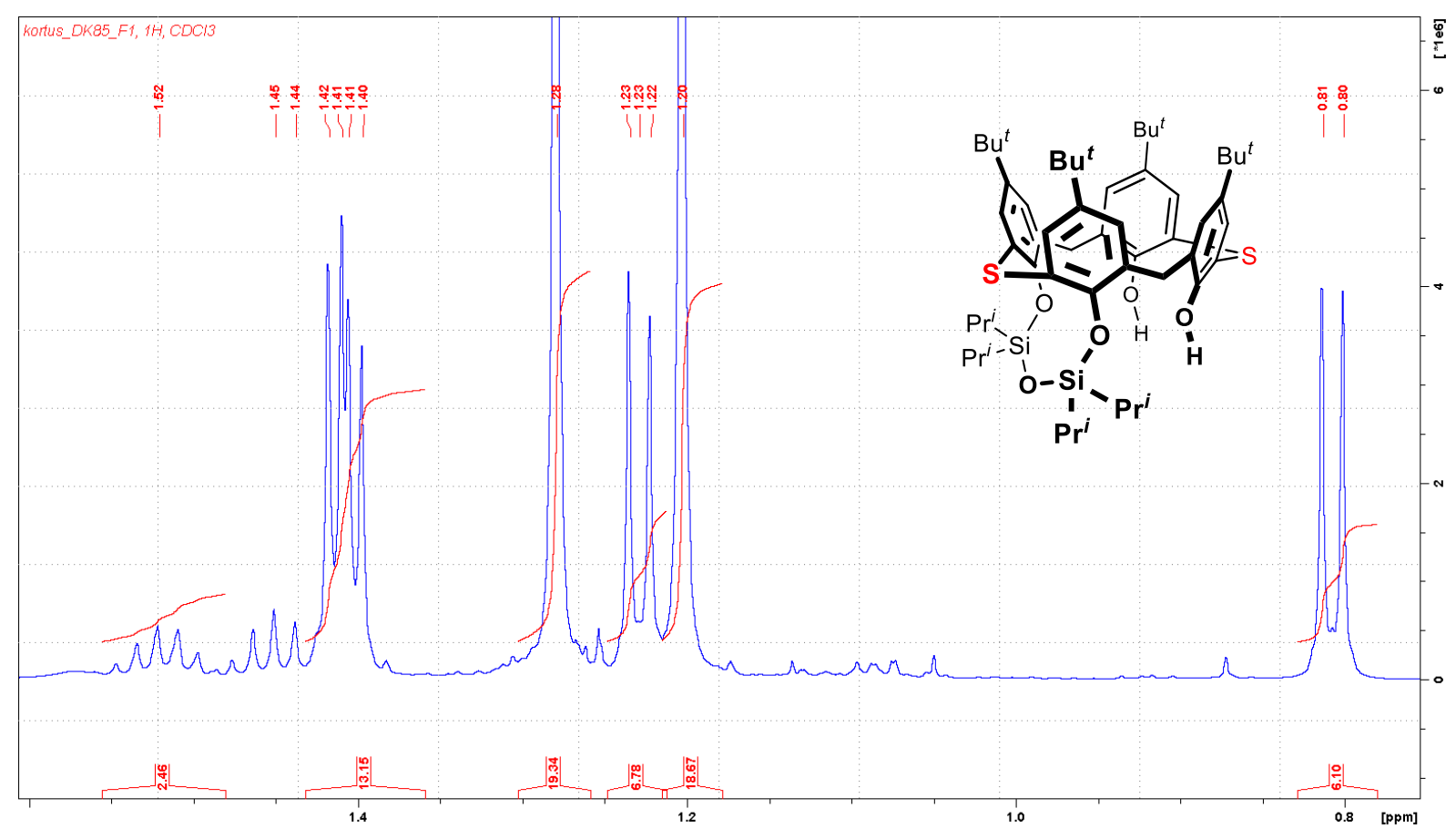

Figure S3. ${ }^{1} \mathrm{H}$ NMR of compound 2, aliphatic region $\left(\mathrm{CD}_{2} \mathrm{Cl}_{2}, 600 \mathrm{MHz}\right)$.

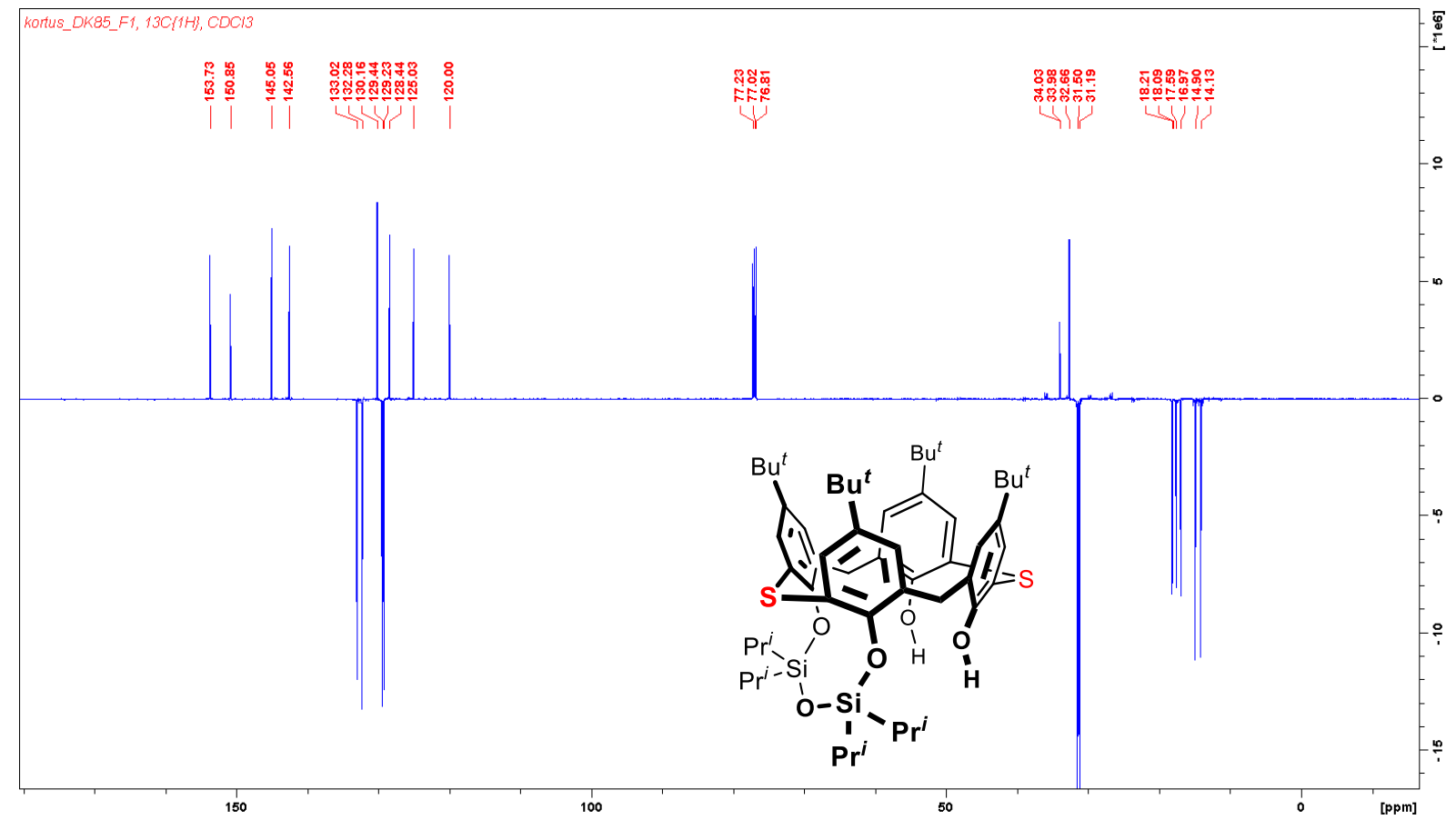

Figure S4. ${ }^{13} \mathrm{C}\left\{{ }^{1} \mathrm{H}\right\}$ NMR of compound $2\left(\mathrm{CD}_{2} \mathrm{Cl}_{2}, 151 \mathrm{MHz}\right)$. 


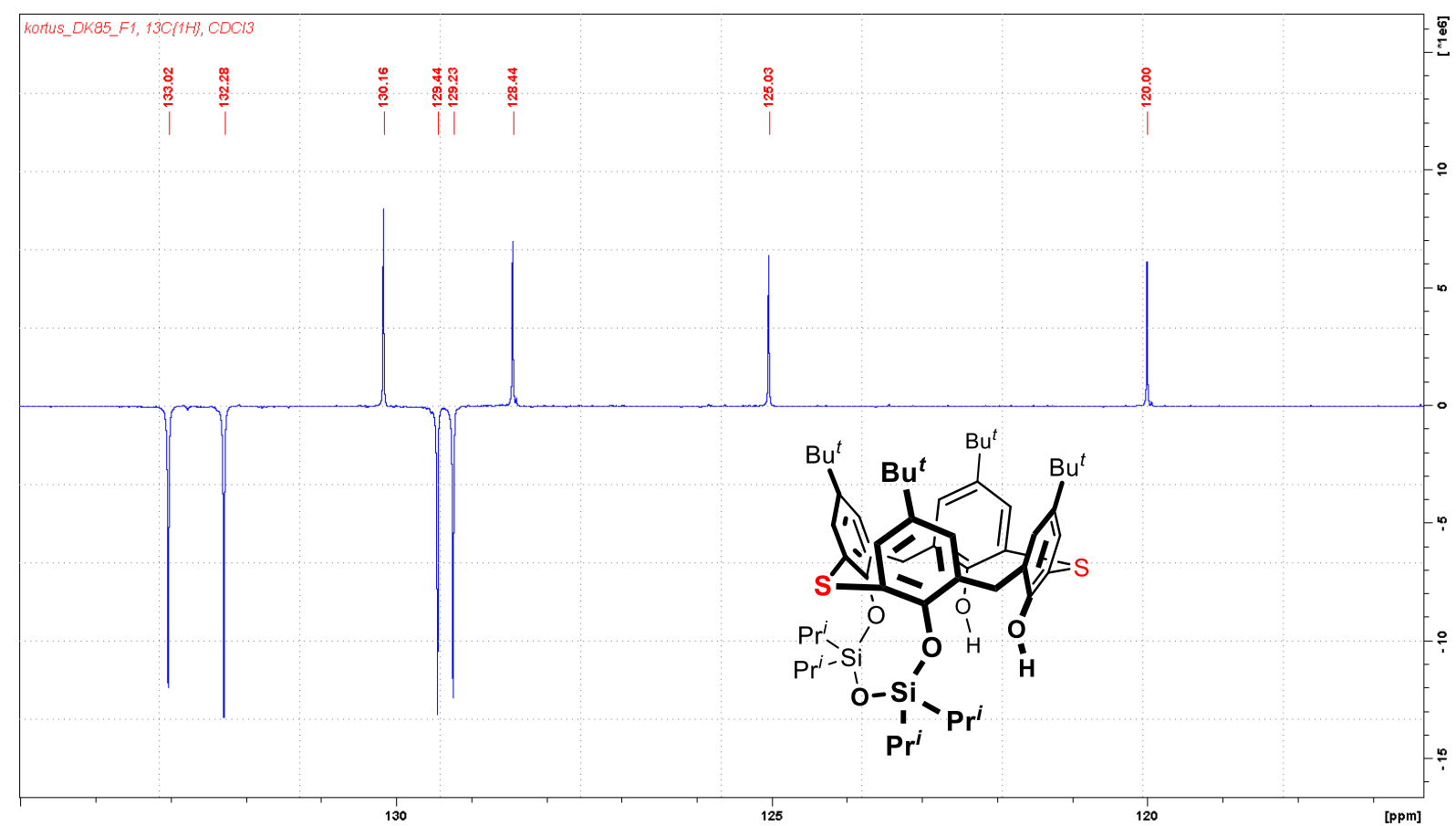

Figure S5. ${ }^{13} \mathrm{C}\left\{{ }^{1} \mathrm{H}\right\}$ NMR of compound 2, aromatic region $\left(\mathrm{CD}_{2} \mathrm{Cl}_{2}, 151 \mathrm{MHz}\right)$.

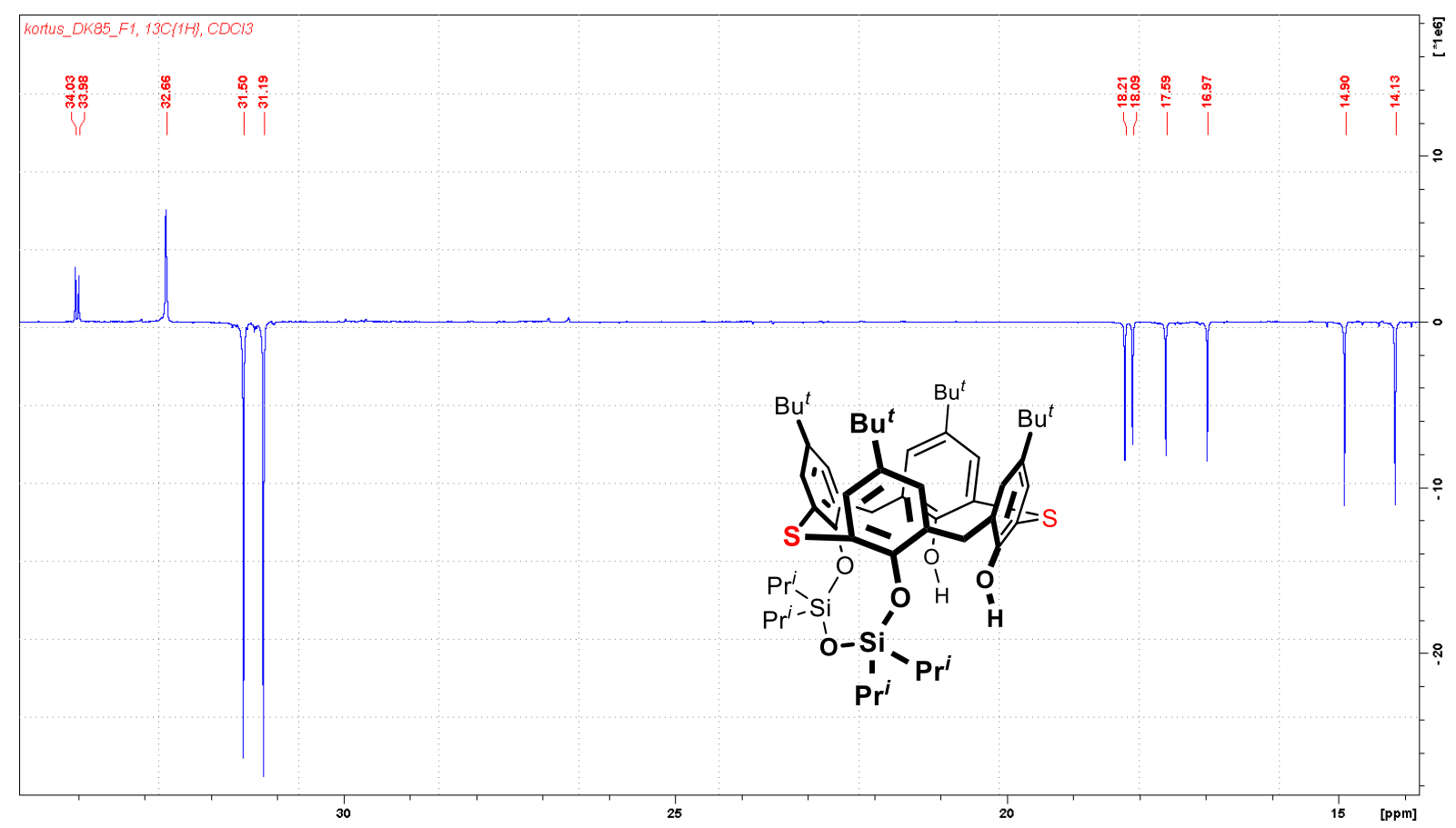

Figure S6. ${ }^{13} \mathrm{C}\left\{{ }^{1} \mathrm{H}\right\}$ NMR of compound 2, aliphatic region $\left(\mathrm{CD}_{2} \mathrm{Cl}_{2}, 151 \mathrm{MHz}\right)$. 


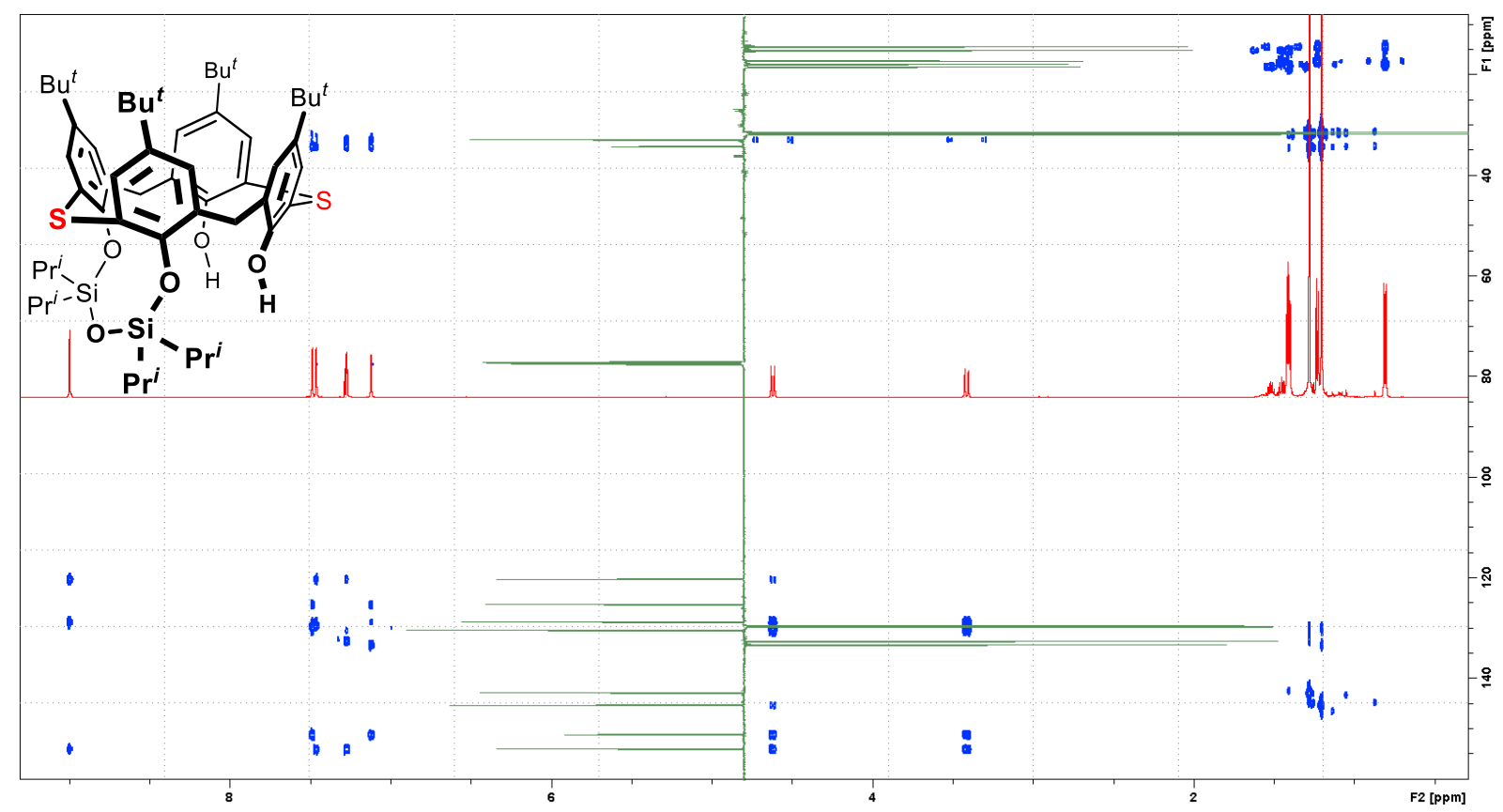

Figure S7. HMBC NMR of compound $2\left(\mathrm{CDCl}_{3}\right)$.

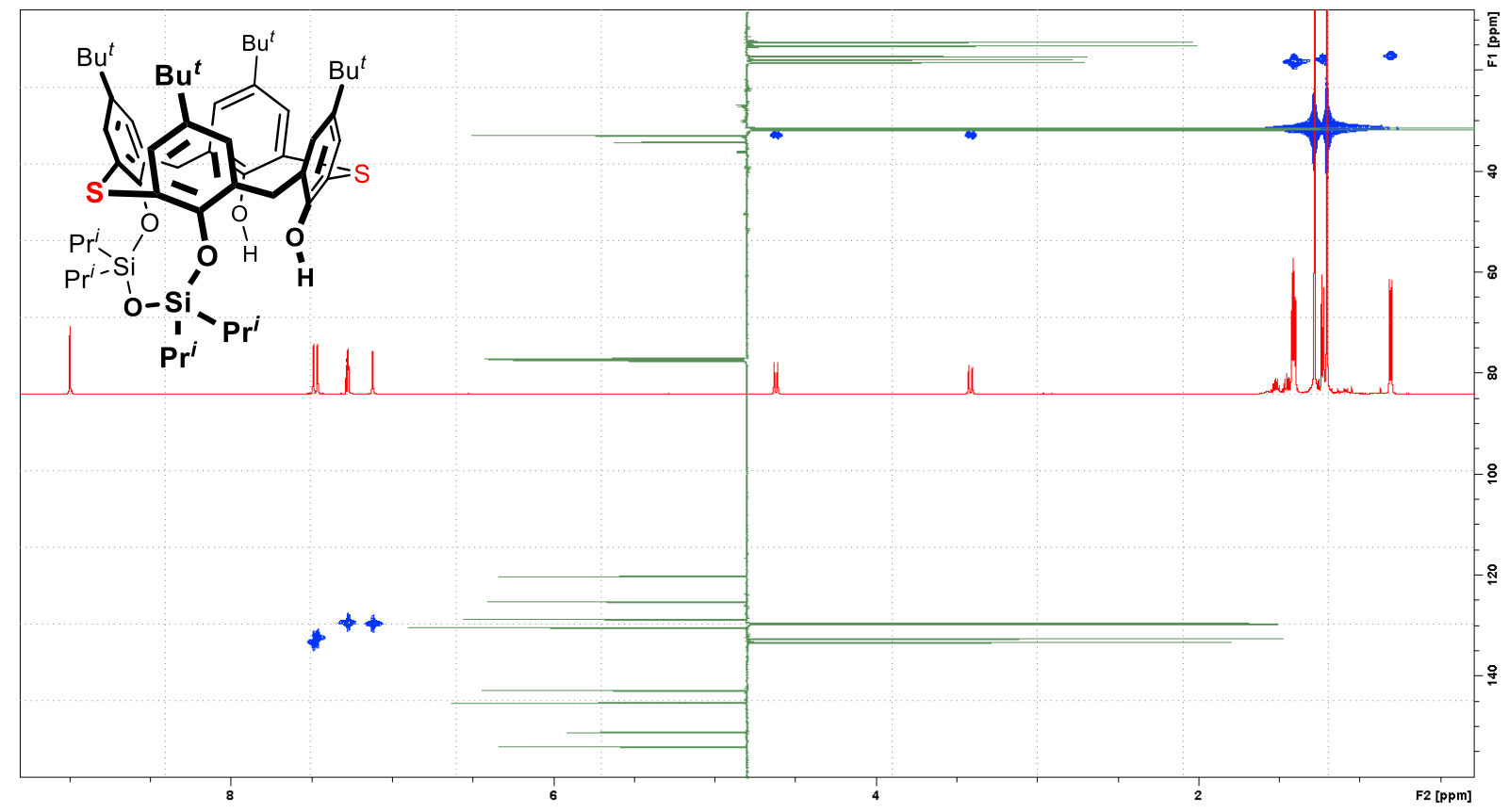

Figure S8. HMQC NMR of compound $2\left(\mathrm{CDCl}_{3}\right)$. 


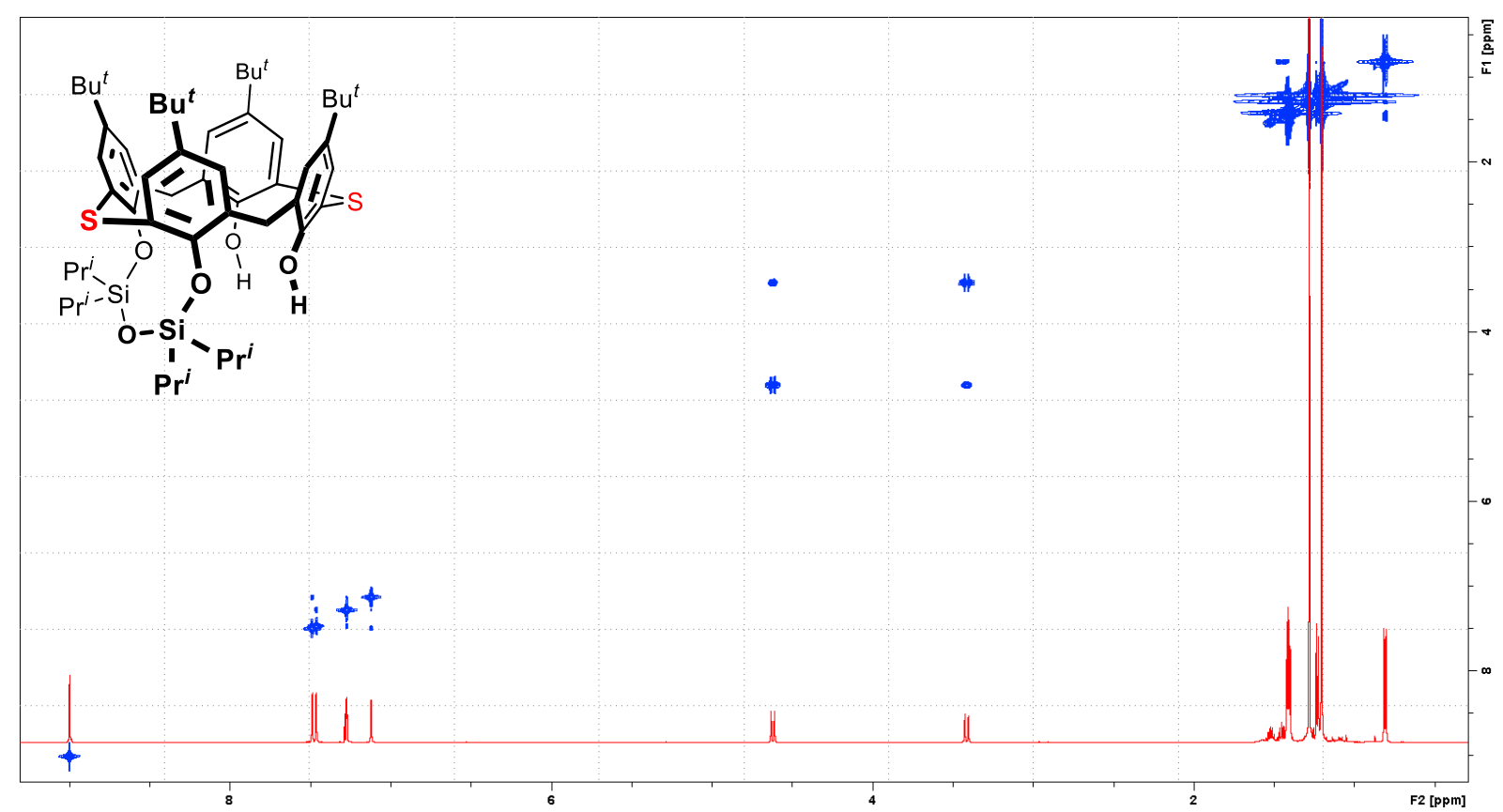

Figure S9. COSY NMR of compound $2\left(\mathrm{CDCl}_{3}\right)$.

117_Kortus_ESIpos_DK76_F1_1 4/2/2019 7:40:25 AM $\mathrm{MeOH}$

117_Kortus_ESIpos_DK76_F1_1 \#82-85 RT: 1.18-1.22 AV: 4 NL: 1.66E7

T: FTMS + c ESI Full ms [200.00-2000.00]

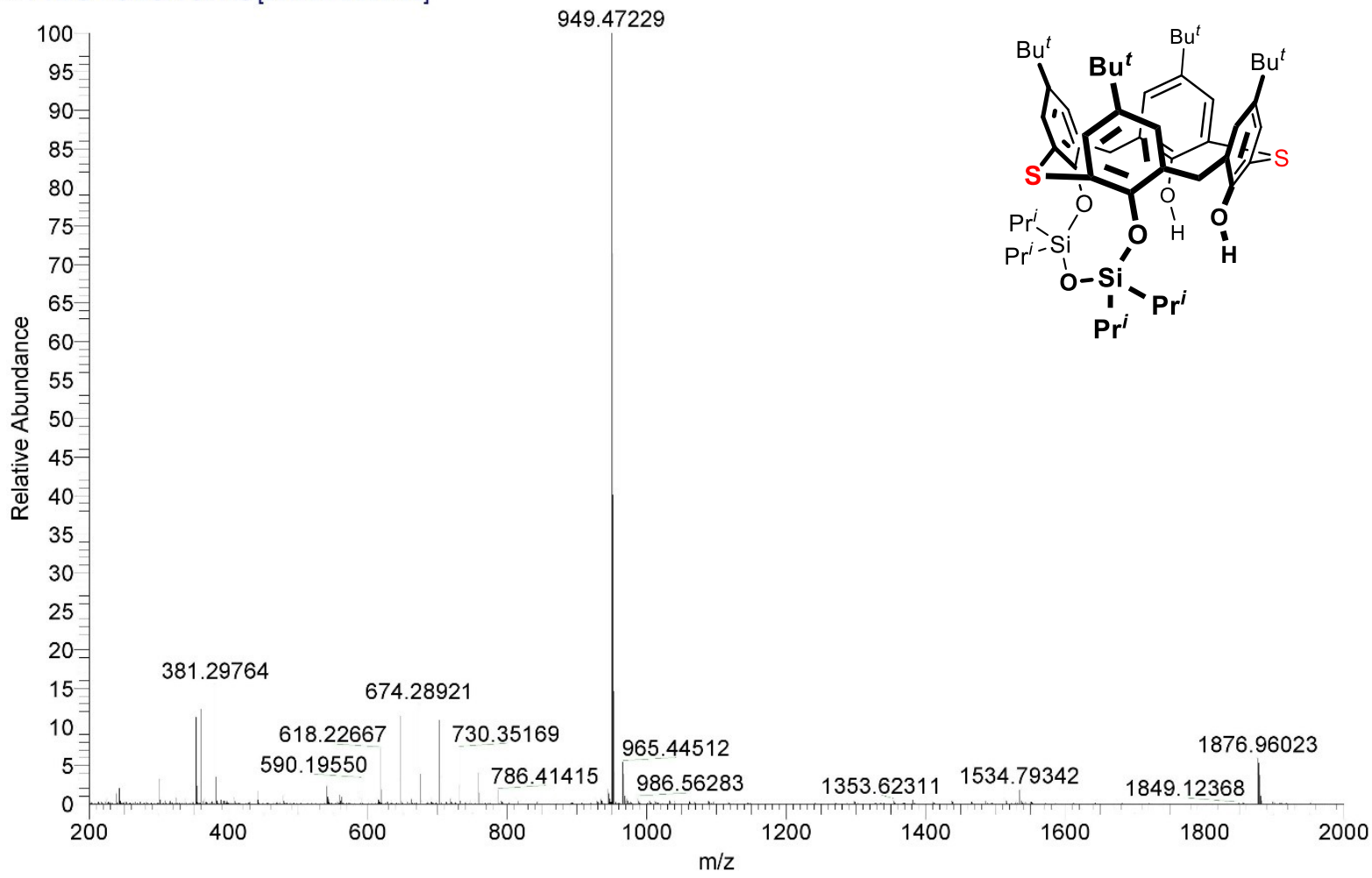

Figure S10. HRMS of compound $2\left(\mathrm{ESI}^{+}\right)$. 


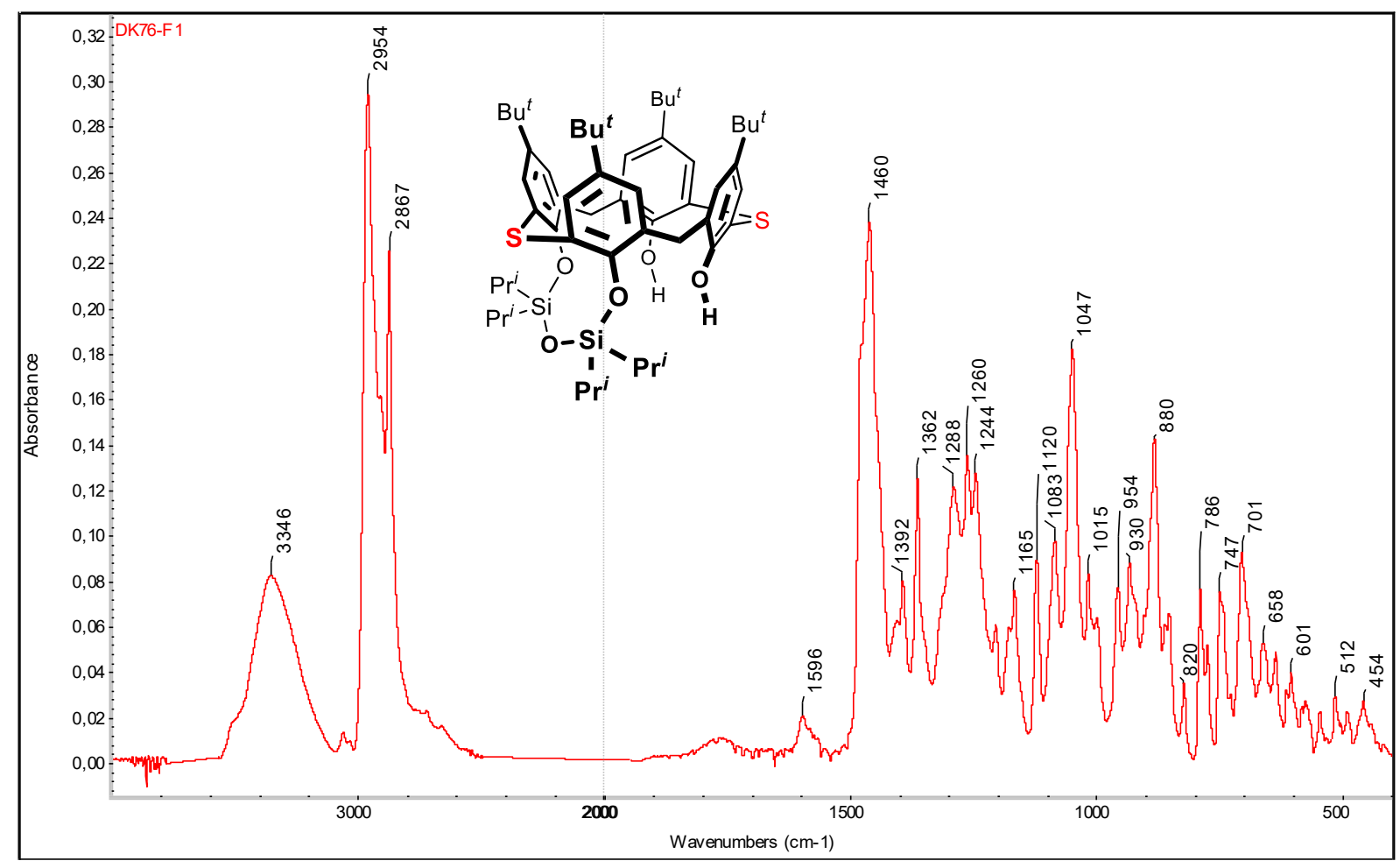

Figure S11. IR of compound 2 (ATR). 


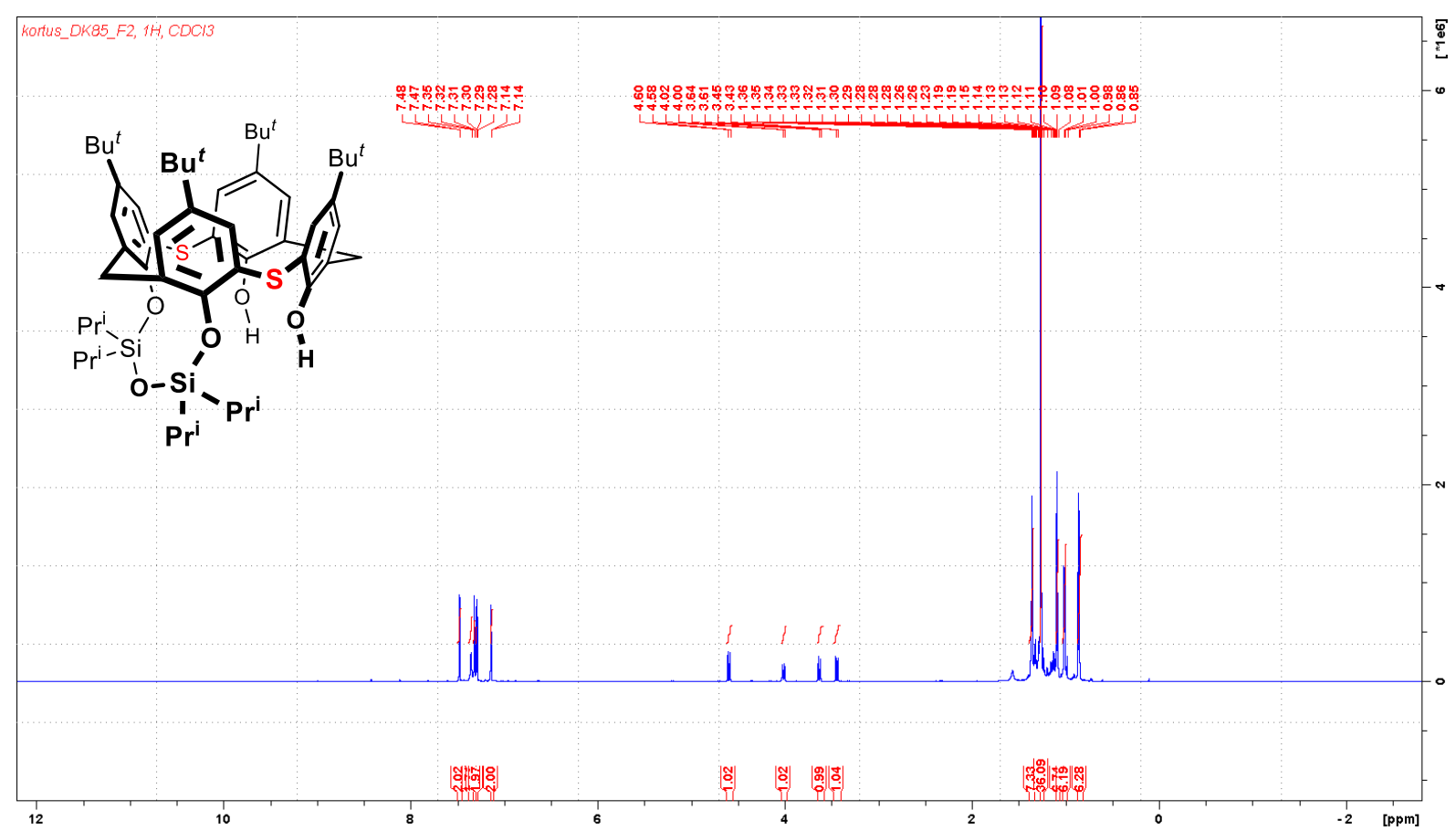

Figure S12. ${ }^{1} \mathrm{H} \mathrm{NMR}$ of compound $3\left(\mathrm{CDCl}_{3}, 600 \mathrm{MHz}\right)$.

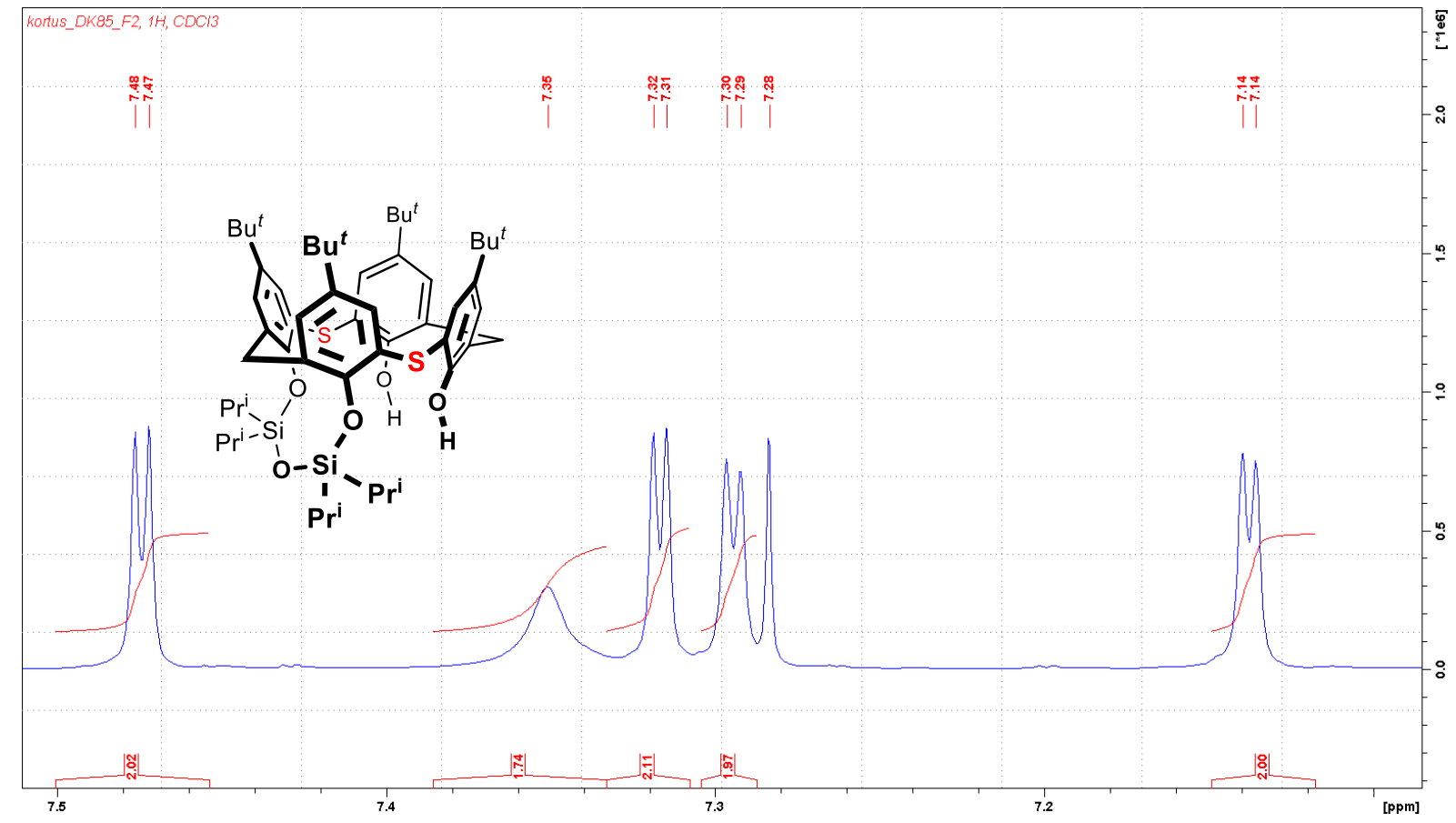

Figure S13. ${ }^{1} \mathrm{H} \mathrm{NMR}$ of compound 3, aromatic region $\left(\mathrm{CDCl}_{3}, 600 \mathrm{MHz}\right)$. 


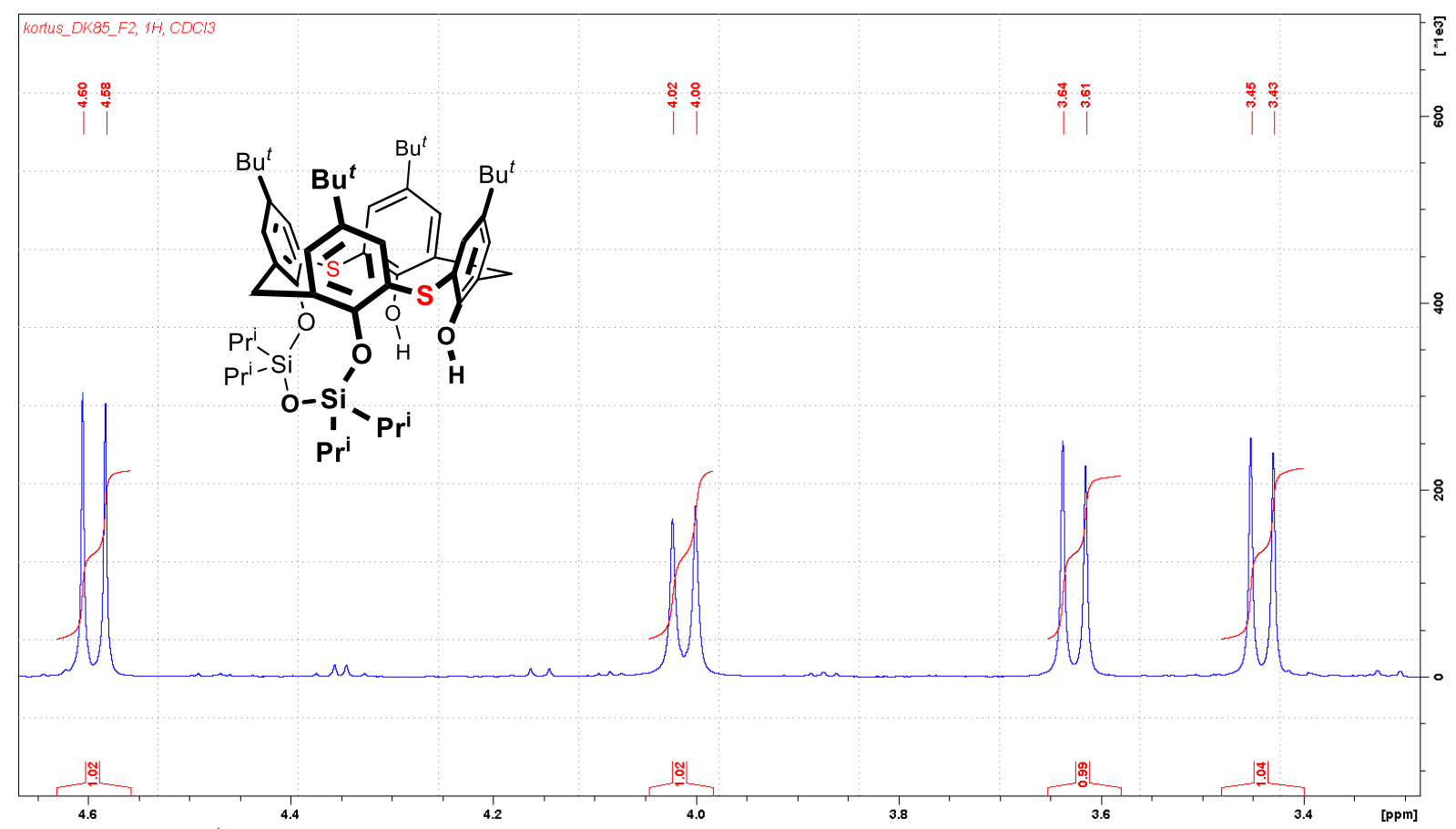

Figure S14. ${ }^{1} \mathrm{H}$ NMR of compound 3, bridge region $\left(\mathrm{CDCl}_{3}, 600 \mathrm{MHz}\right)$.

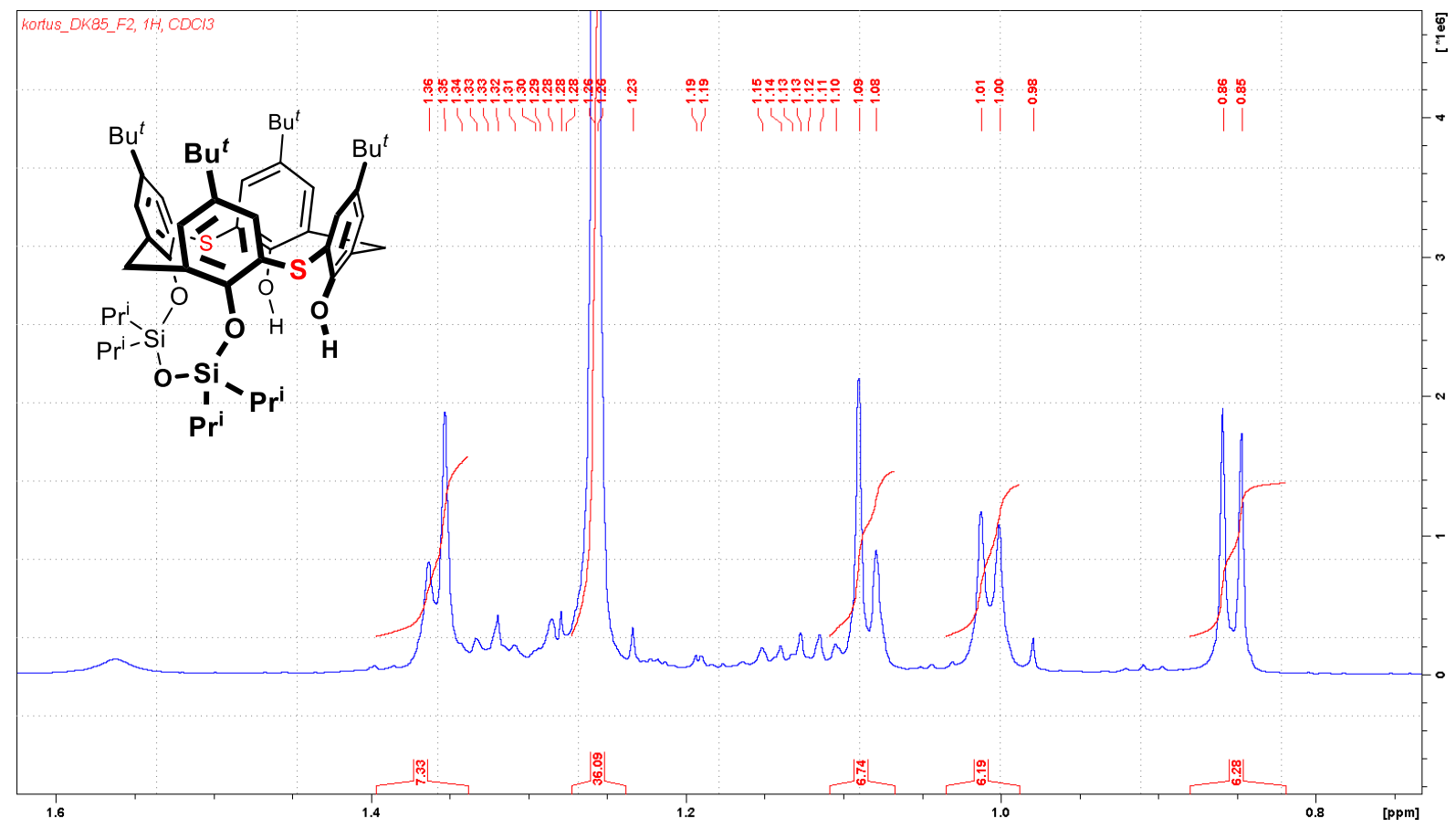

Figure S15. ${ }^{1} \mathrm{H} \mathrm{NMR}$ of compound 3, aliphatic region $\left(\mathrm{CDCl}_{3}, 600 \mathrm{MHz}\right)$. 


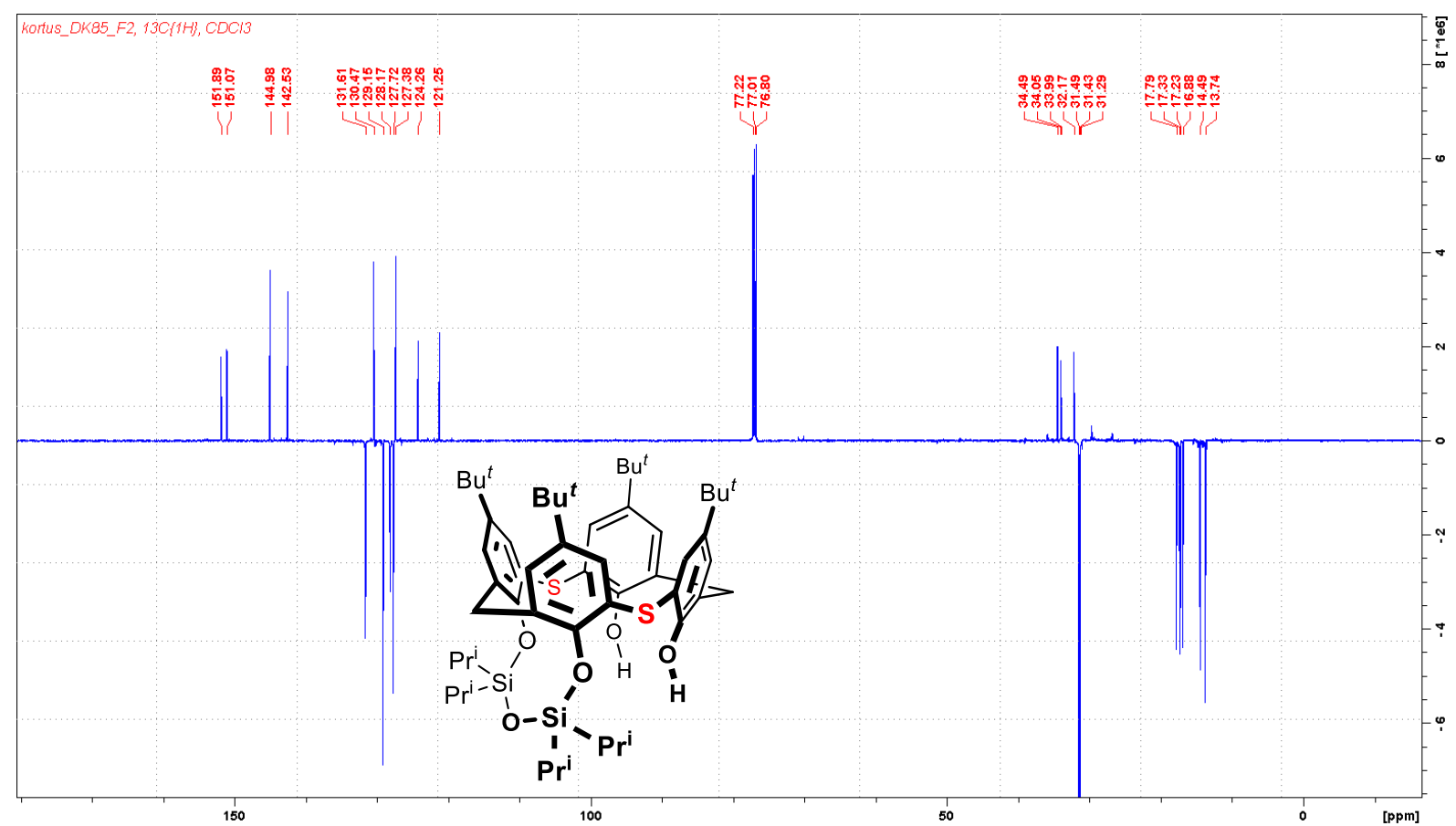

Figure S16. ${ }^{13} \mathrm{C}\left\{{ }^{1} \mathrm{H}\right\} \mathrm{NMR}$ of compound $3\left(\mathrm{CDCl}_{3}, 151 \mathrm{MHz}\right)$.

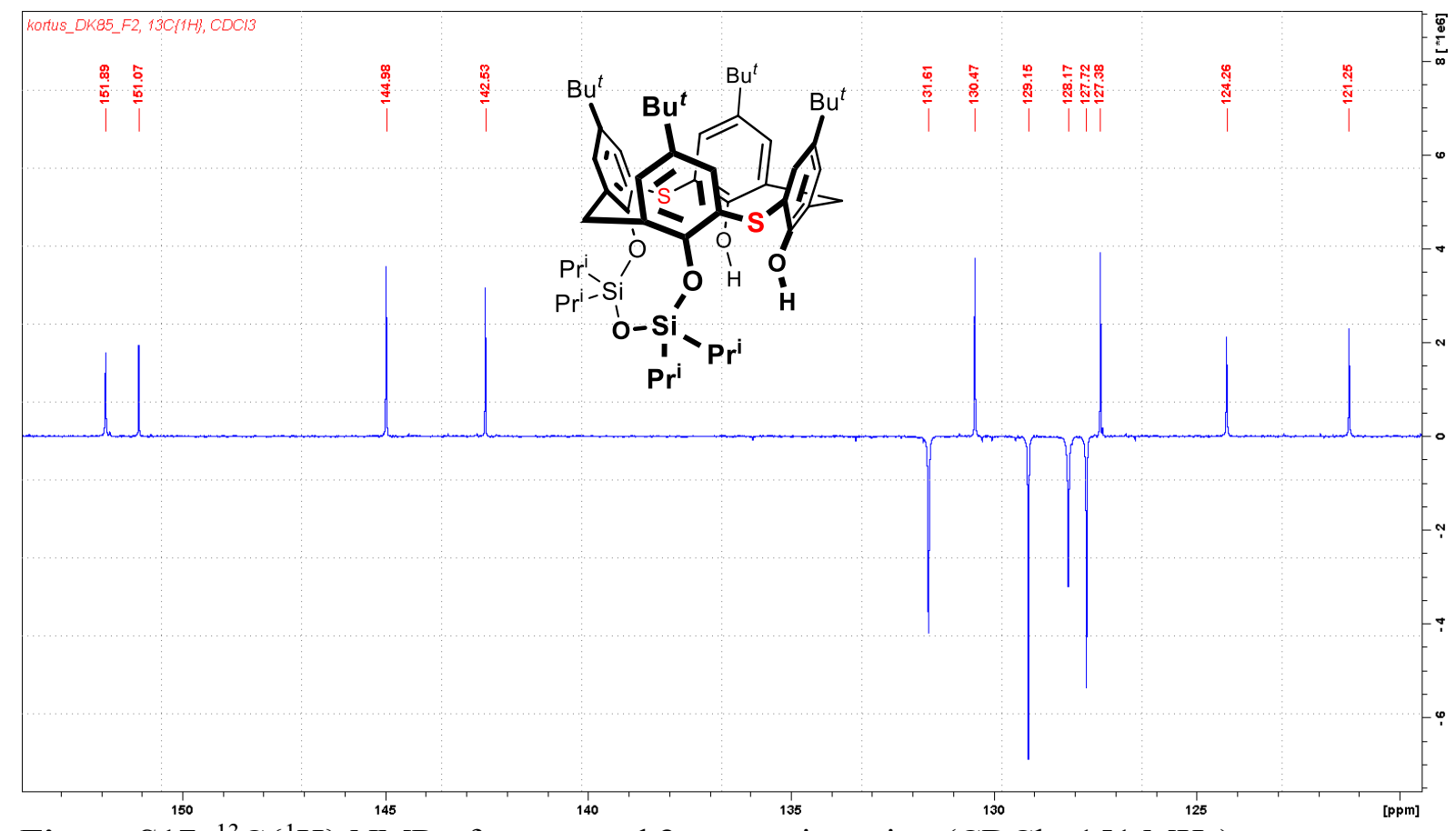

Figure S17. ${ }^{13} \mathrm{C}\left\{{ }^{1} \mathrm{H}\right\}$ NMR of compound 3, aromatic region $\left(\mathrm{CDCl}_{3}, 151 \mathrm{MHz}\right)$. 


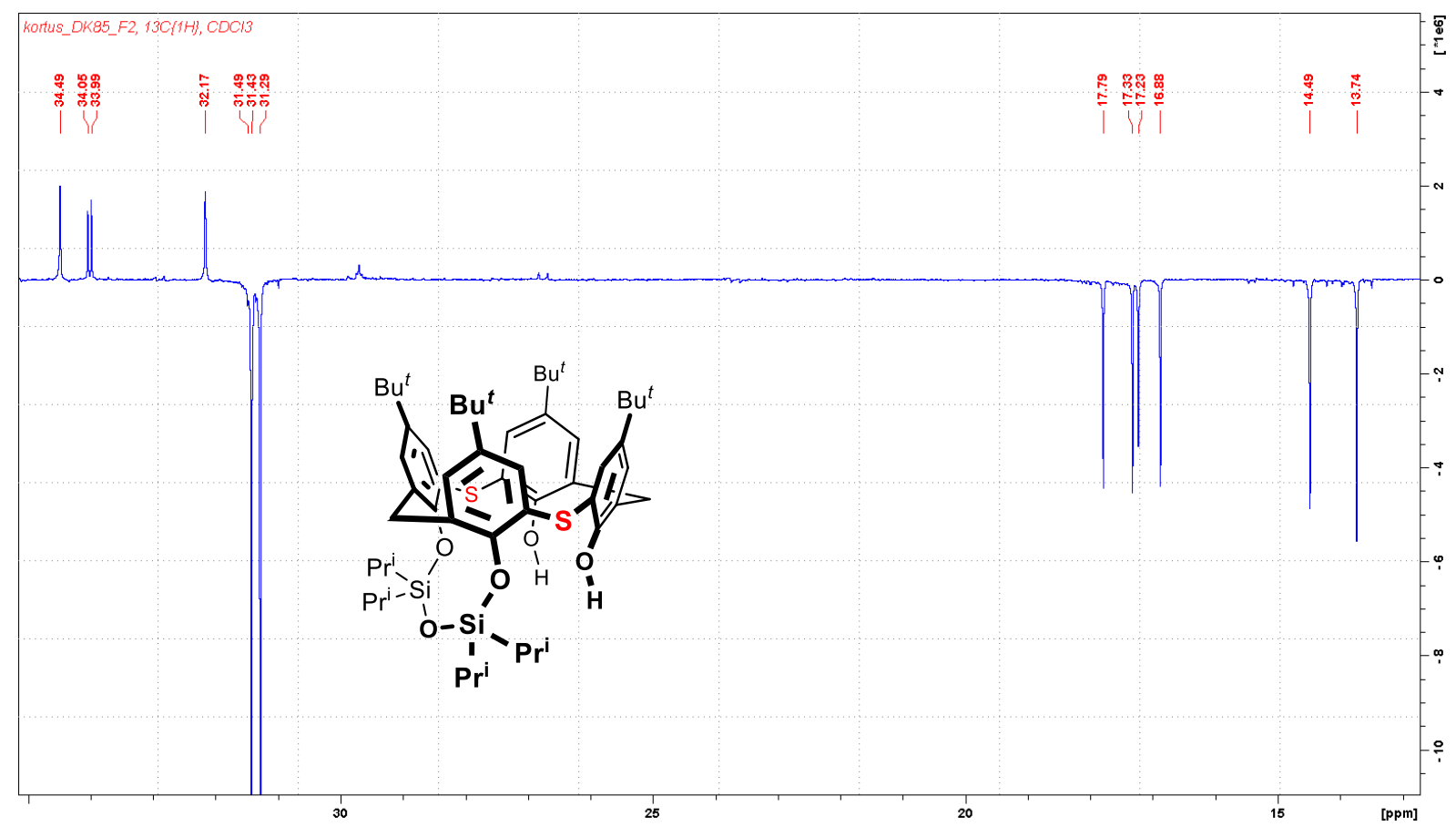

Figure S18. ${ }^{13} \mathrm{C}\left\{{ }^{1} \mathrm{H}\right\}$ NMR of compound 3, aliphatic region $\left(\mathrm{CDCl}_{3}, 151 \mathrm{MHz}\right)$.

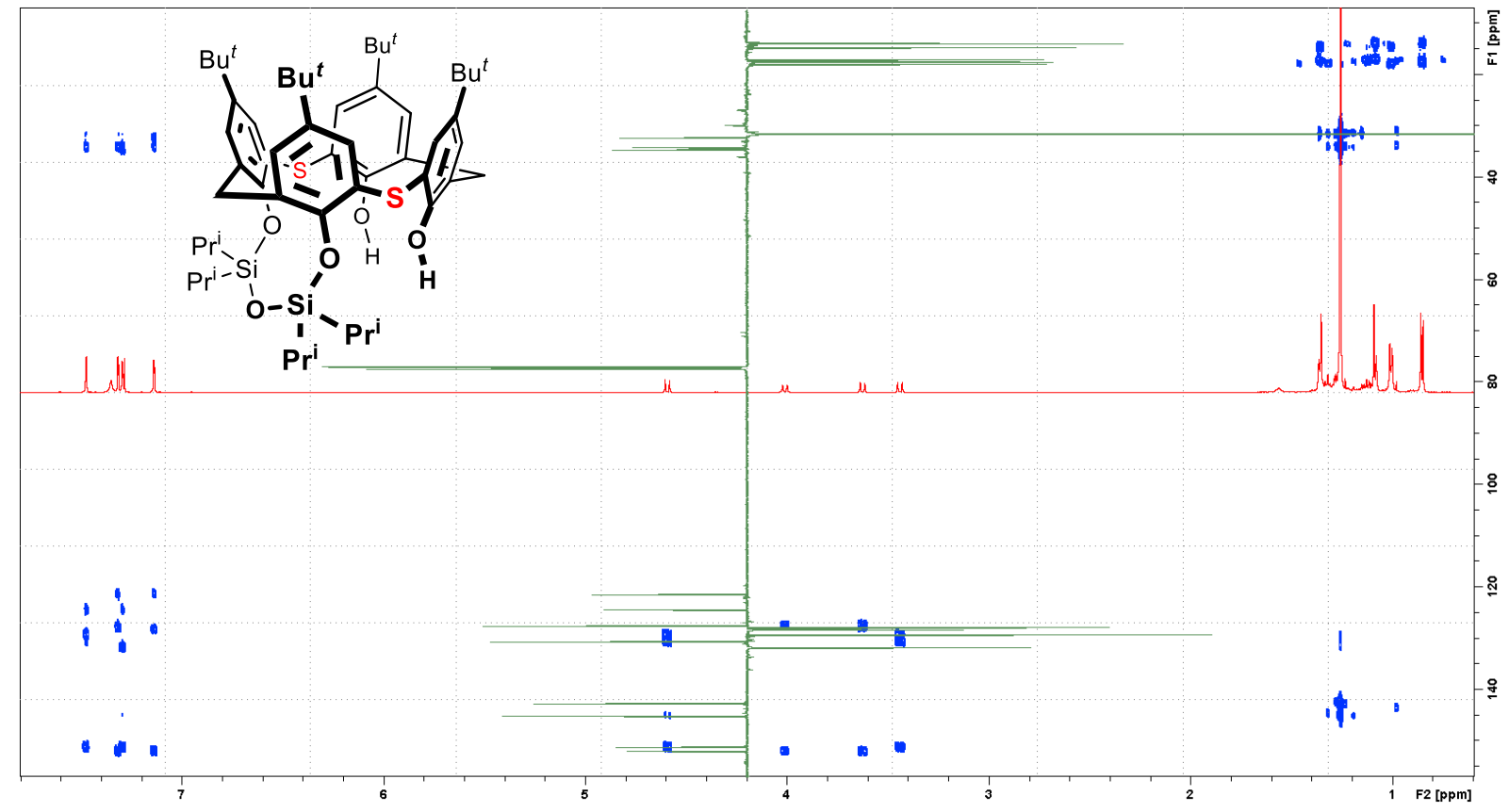

Figure S19. HMBC NMR of compound $3\left(\mathrm{CDCl}_{3}\right)$. 


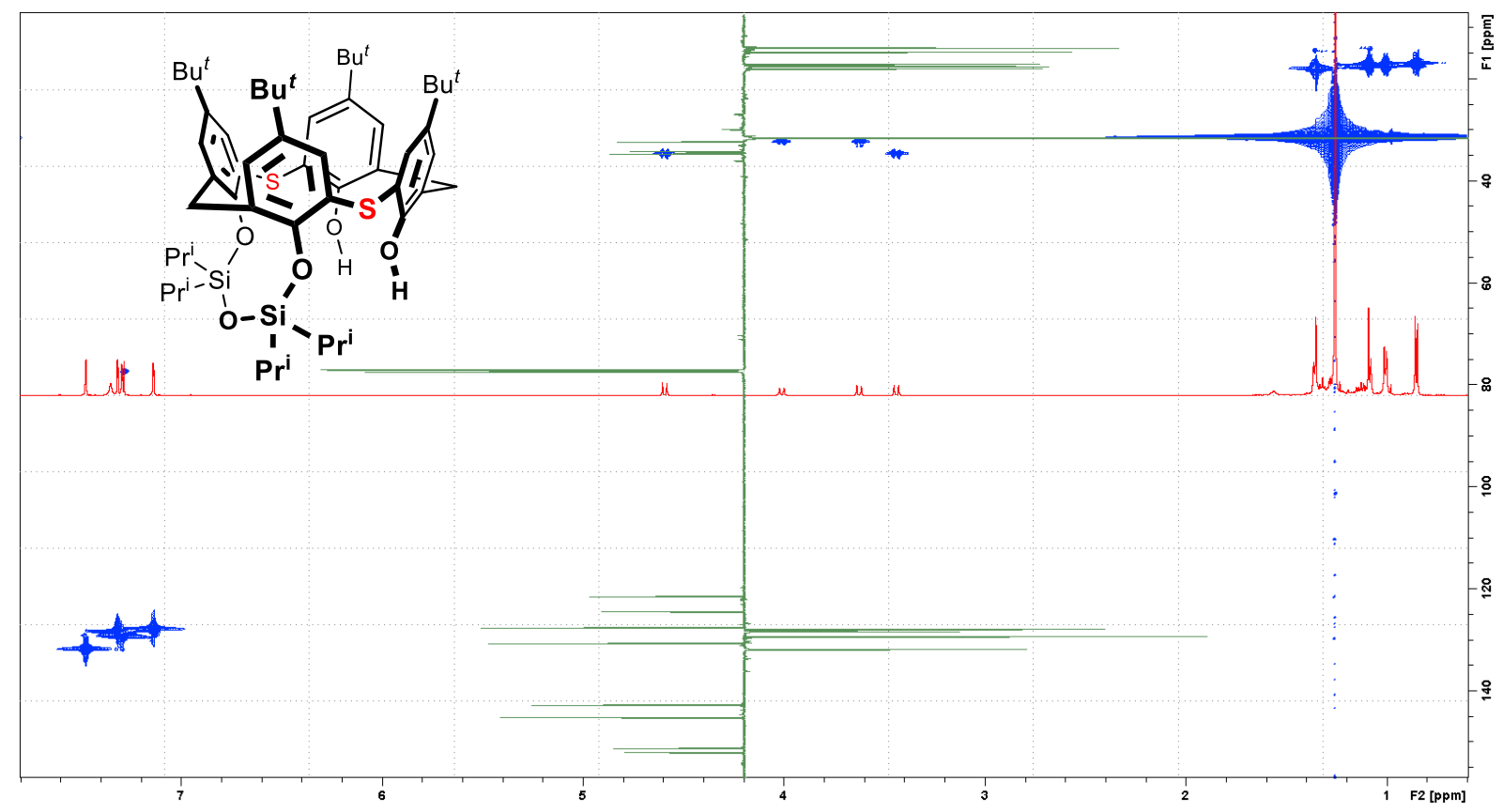

Figure S20. HMQC NMR of compound $3\left(\mathrm{CDCl}_{3}\right)$.

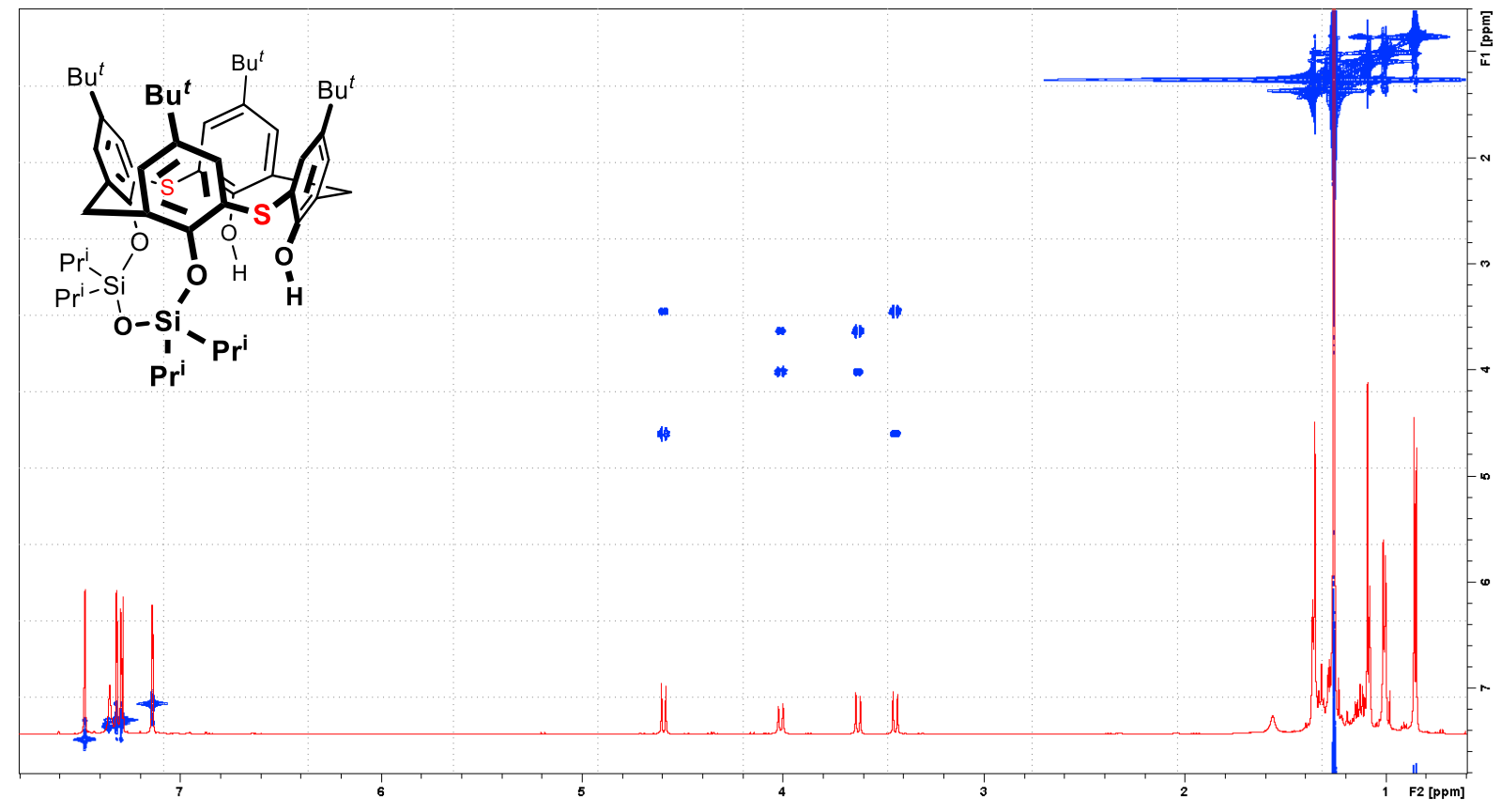

Figure S21. COSY NMR of compound $3\left(\mathrm{CDCl}_{3}\right)$. 
117 Kortus_ESIpos_DK76_F2_1

4/2/2019 7:43:50 AM

117 Kortus ESIpos DK76 F2 1 \#50-54 RT: 0.72-0.78 AV: 5 NL: $1.83 E 7$

T: FTMS + c ESI Full ms [200.00-2000.00]

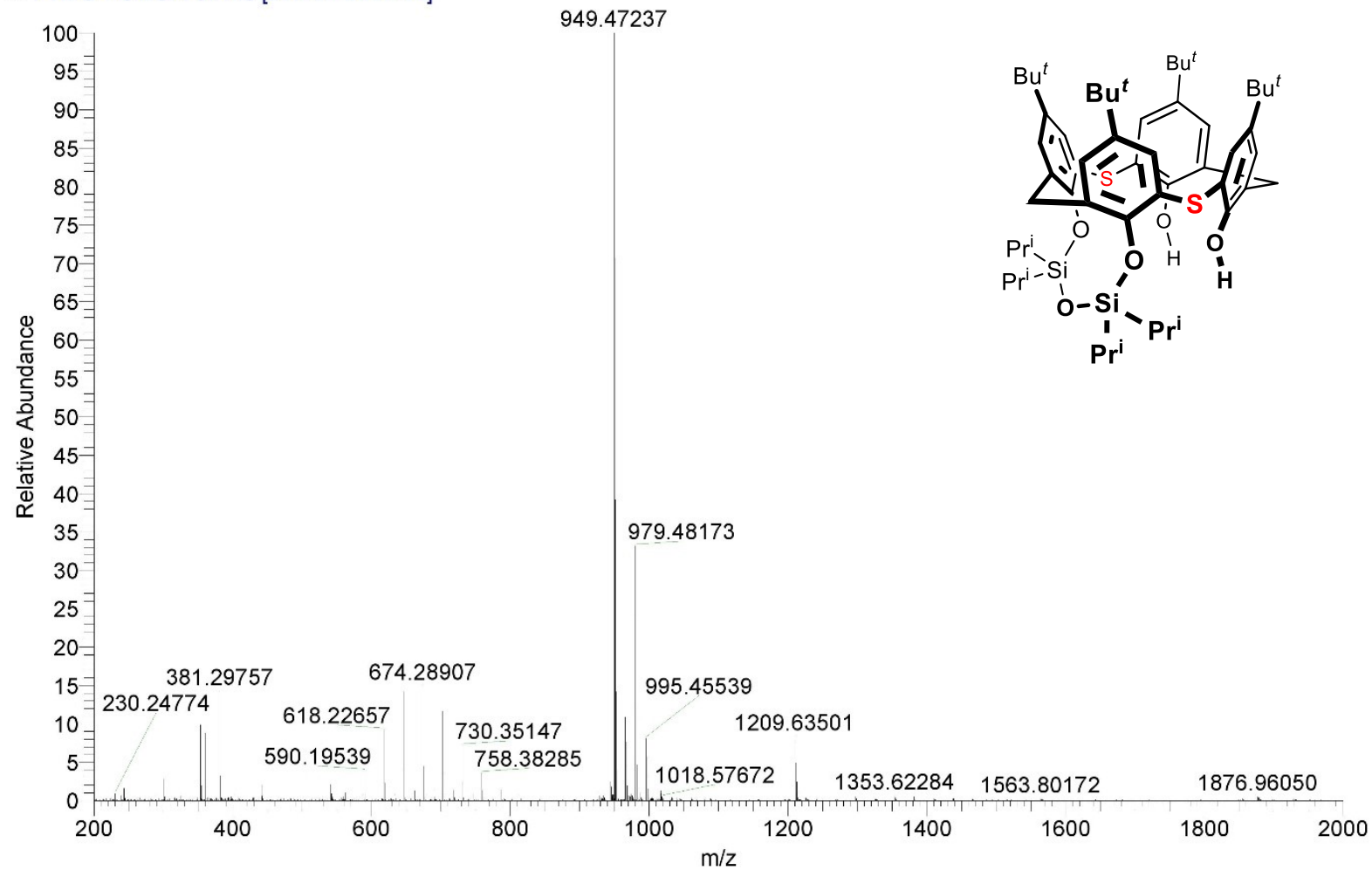

Figure S22. HRMS of compound $3\left(\mathrm{ESI}^{+}\right)$.

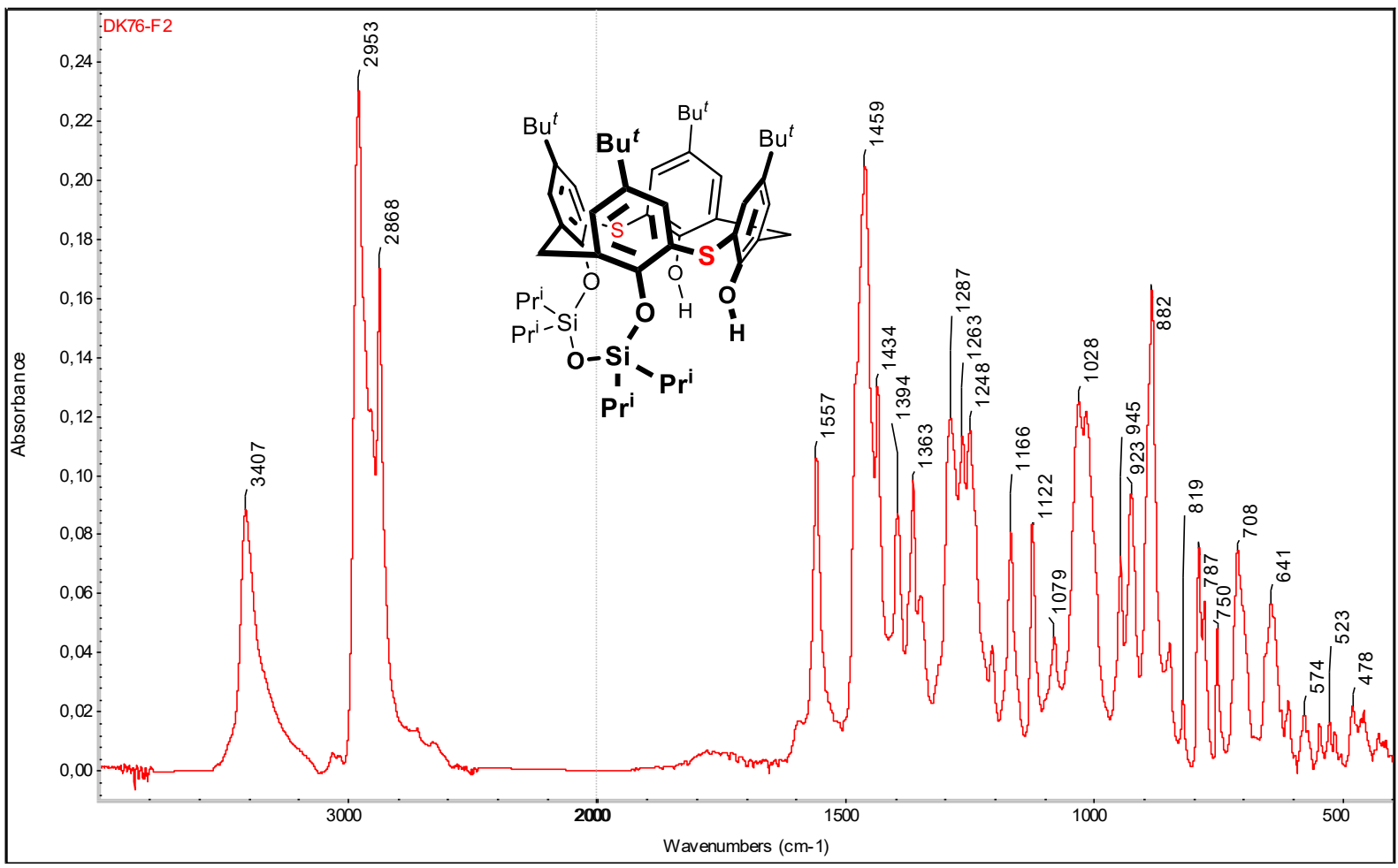

Figure S23. IR of compound 3 (ATR). 


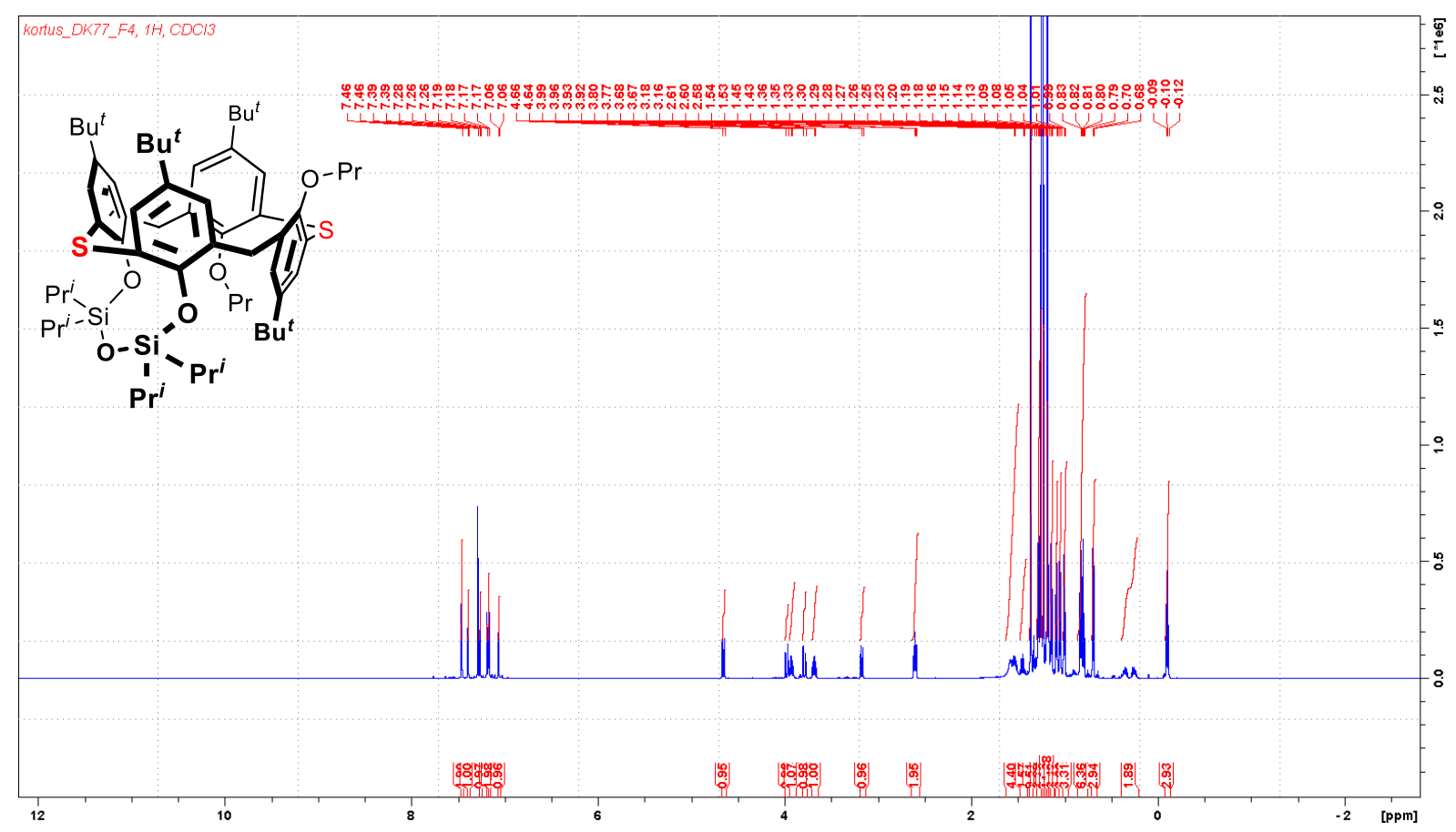

Figure S24. ${ }^{1} \mathrm{H} \mathrm{NMR}$ of compound $4\left(\mathrm{CDCl}_{3}, 600 \mathrm{MHz}\right)$.

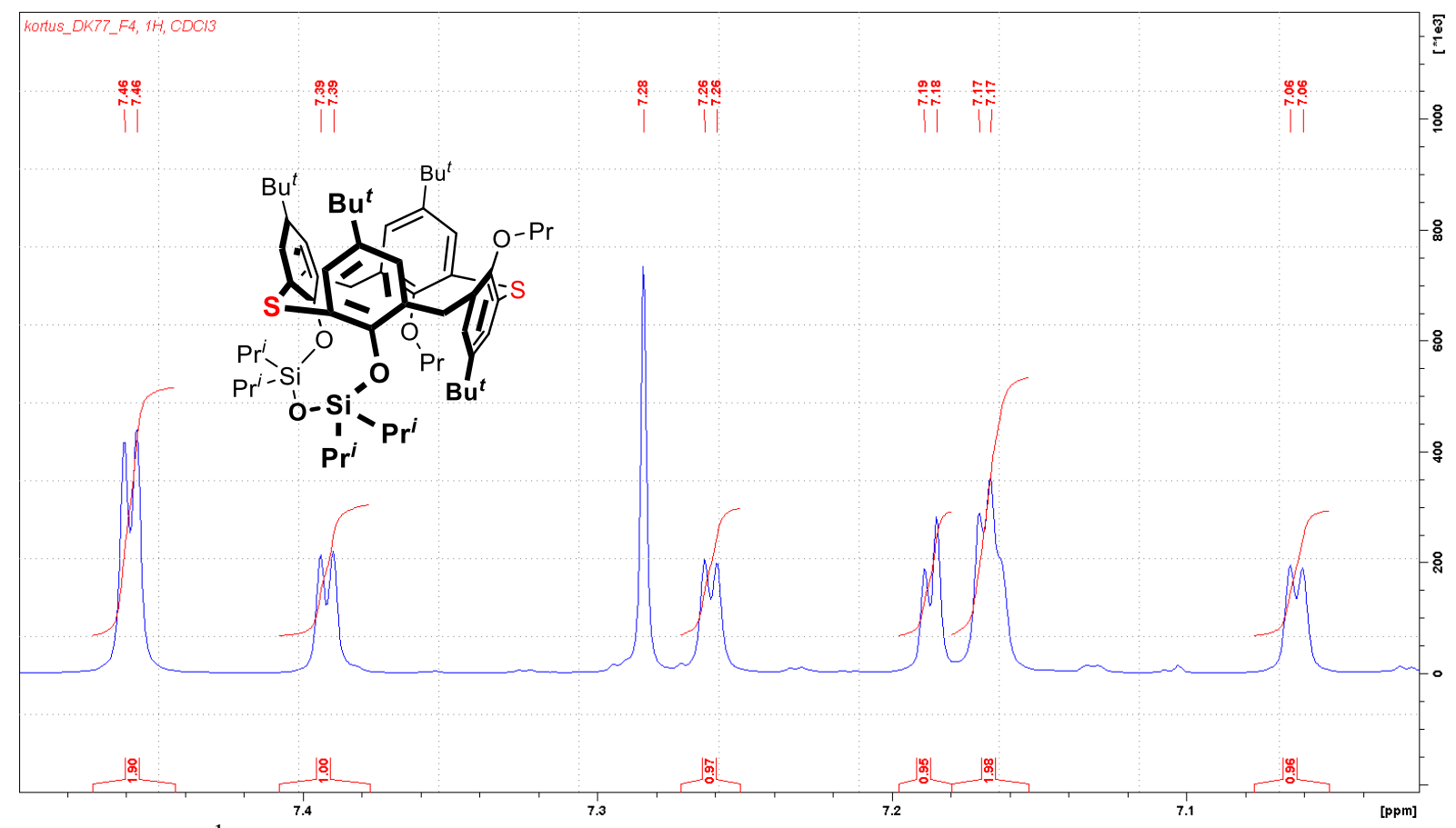

Figure S25. ${ }^{1} \mathrm{H}$ NMR of compound 4, aromatic region $\left(\mathrm{CDCl}_{3}, 600 \mathrm{MHz}\right)$. 


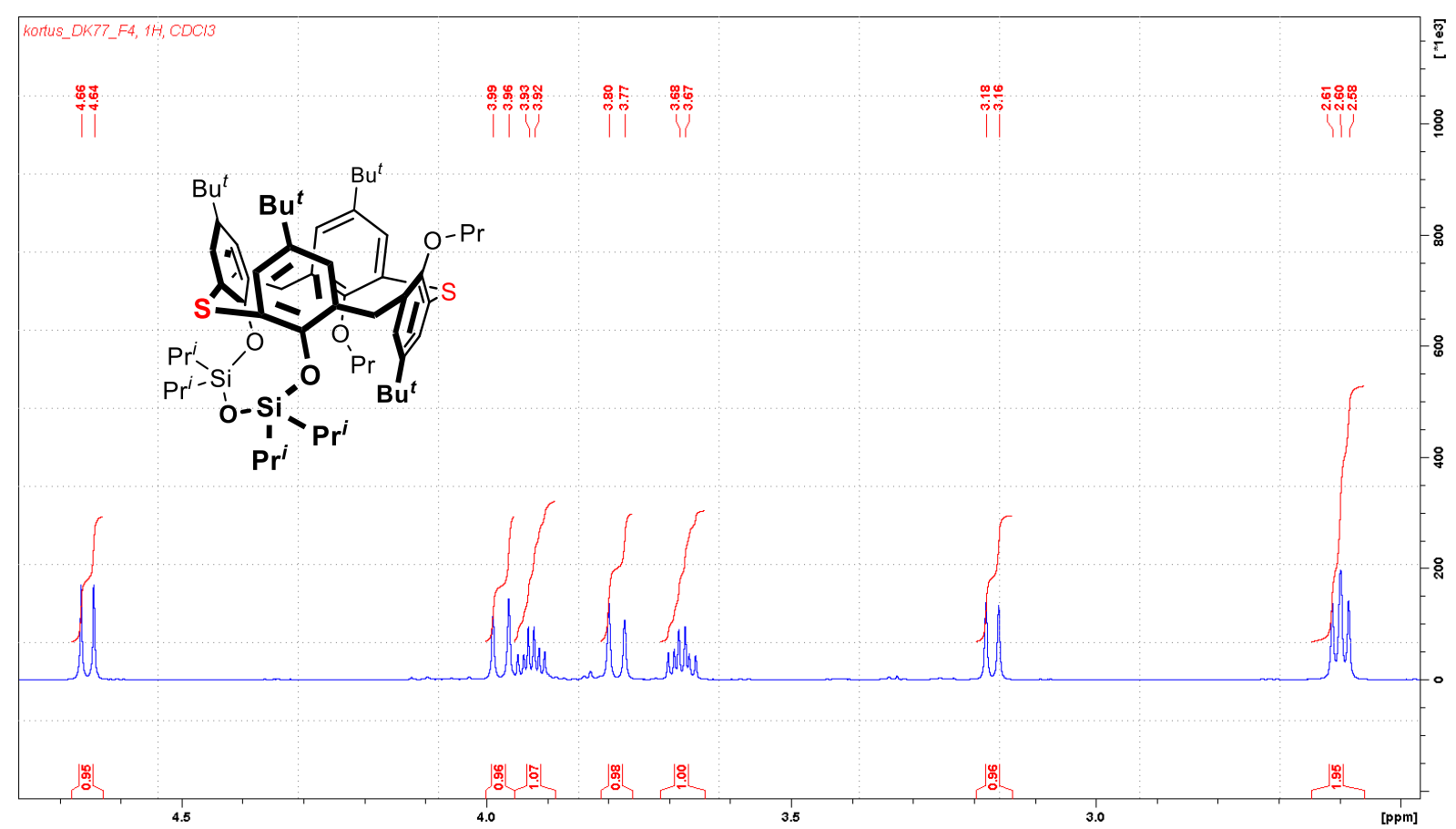

Figure S26. ${ }^{1} \mathrm{H}$ NMR of compound 4, bridge region $\left(\mathrm{CDCl}_{3}, 600 \mathrm{MHz}\right)$.

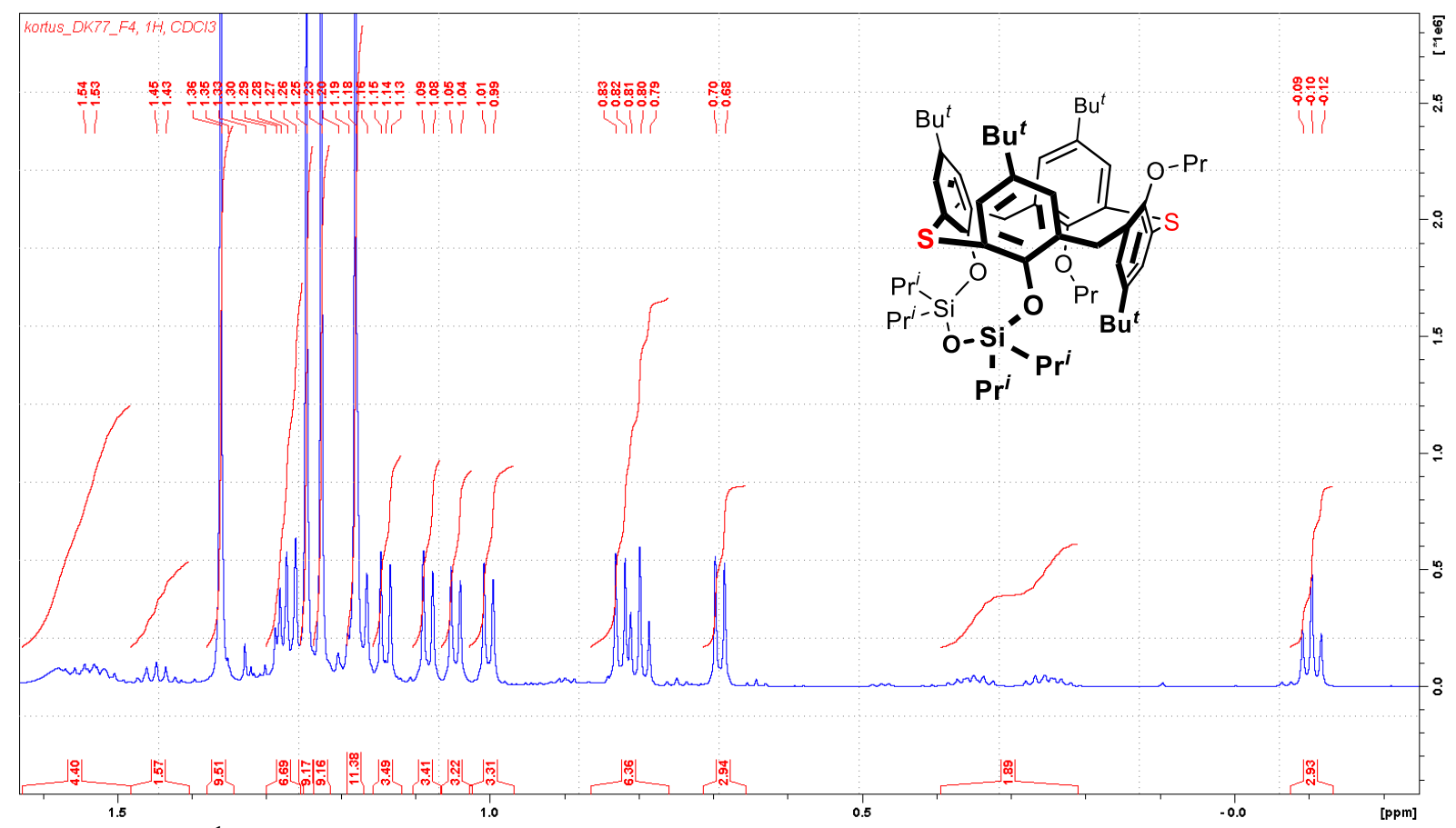

Figure S27. ${ }^{1} \mathrm{H}$ NMR of compound 4, aliphatic region $\left(\mathrm{CDCl}_{3}, 600 \mathrm{MHz}\right)$. 


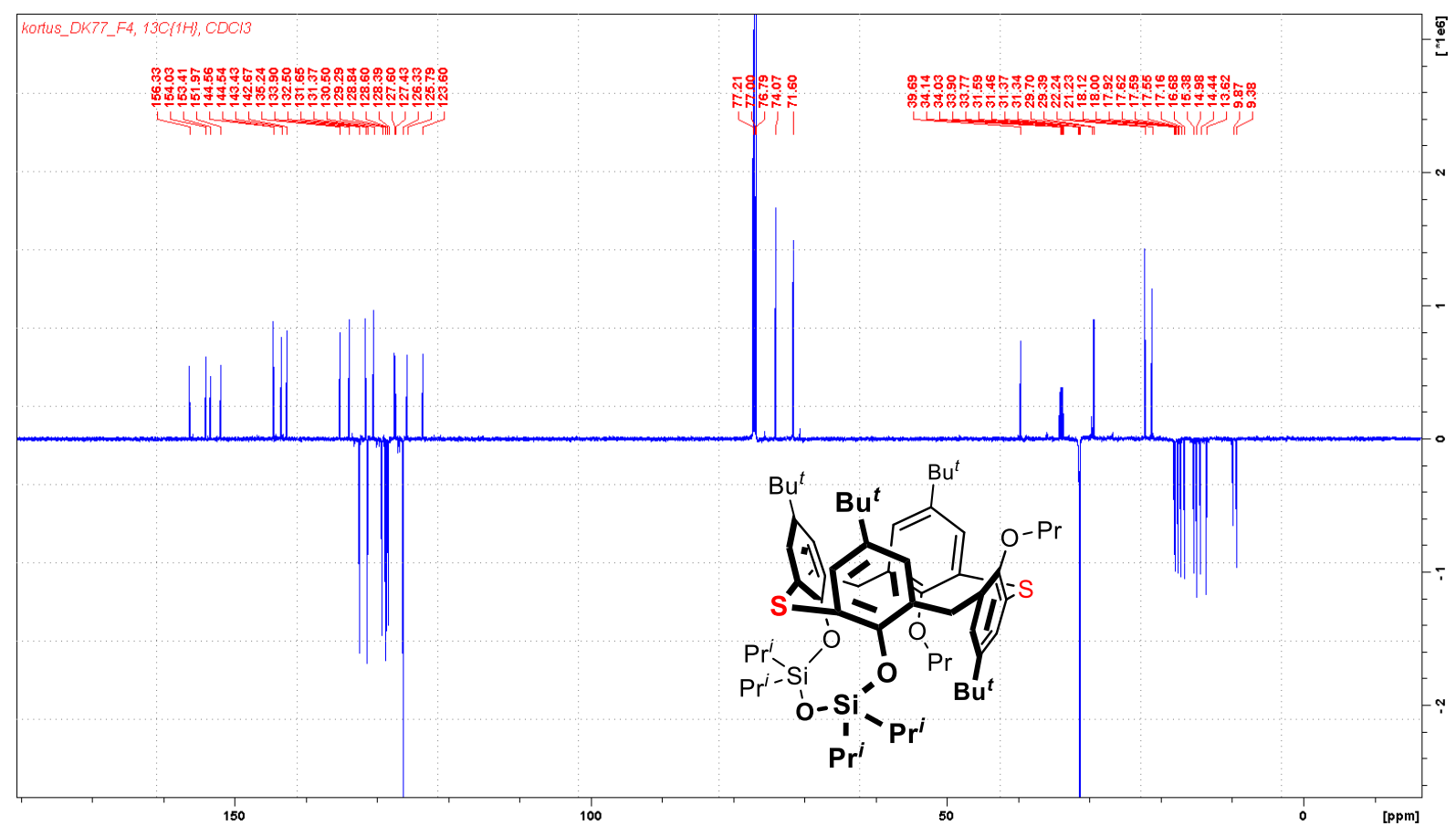

Figure S28. ${ }^{13} \mathrm{C}\left\{{ }^{1} \mathrm{H}\right\}$ NMR of compound $4\left(\mathrm{CDCl}_{3}, 151 \mathrm{MHz}\right)$.

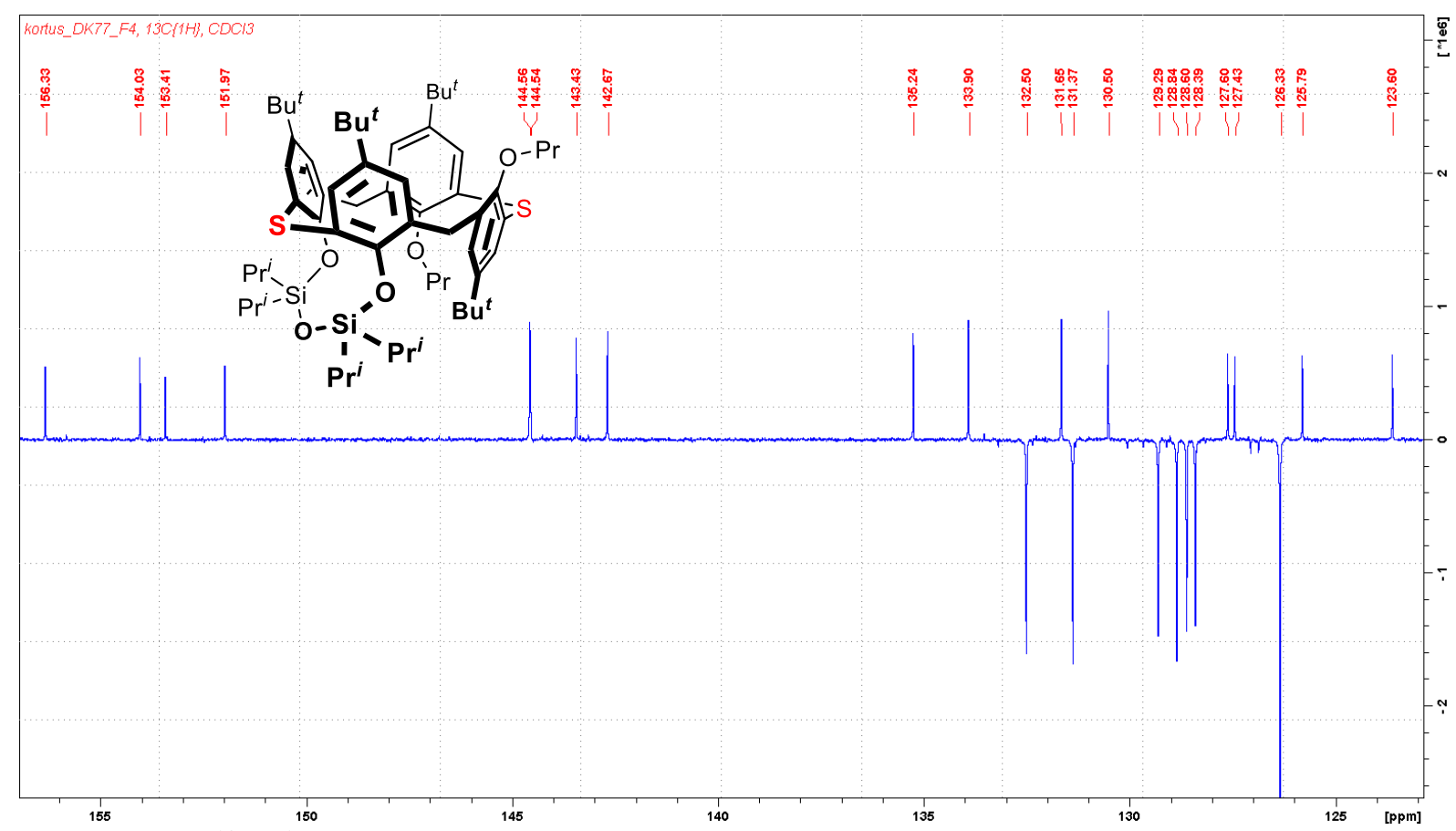

Figure S29. ${ }^{13} \mathrm{C}\left\{{ }^{1} \mathrm{H}\right\}$ NMR of compound 4, aromatic region $\left(\mathrm{CDCl}_{3}, 151 \mathrm{MHz}\right)$. 


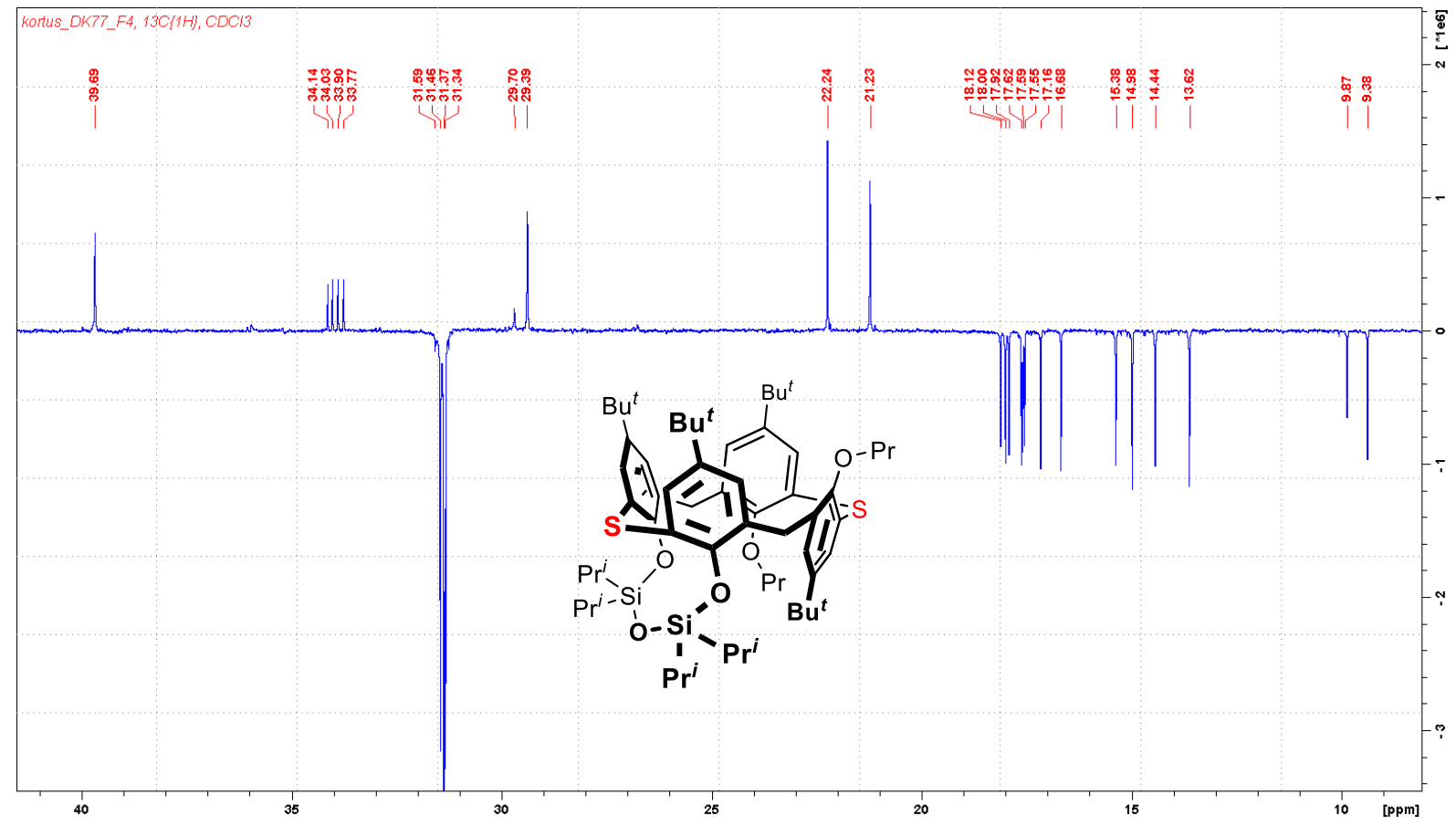

Figure S30. ${ }^{13} \mathrm{C}\left\{{ }^{1} \mathrm{H}\right\}$ NMR of compound 4, aliphatic region $\left(\mathrm{CDCl}_{3}, 151 \mathrm{MHz}\right)$.

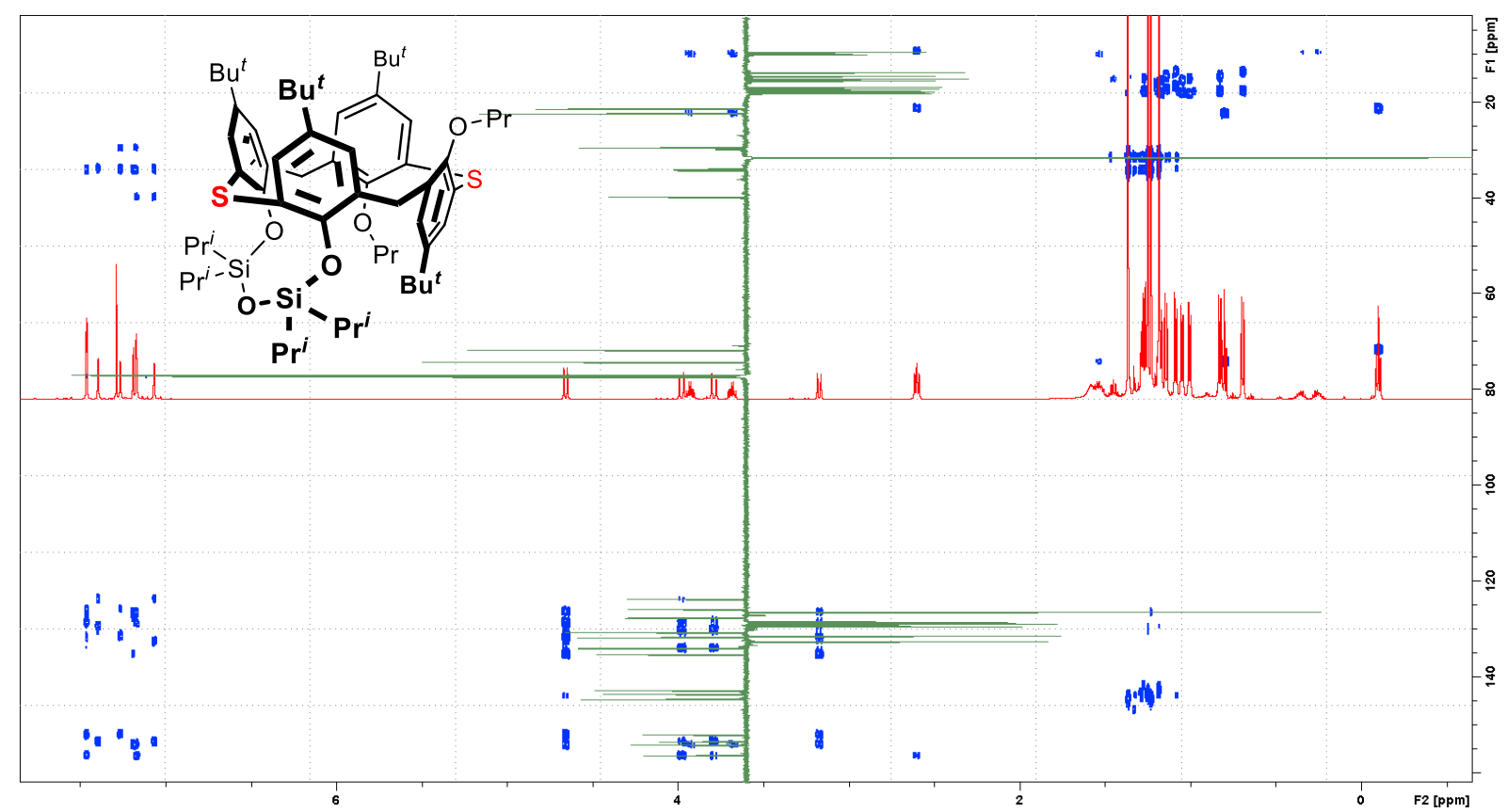

Figure S31. HMBC NMR of compound $4\left(\mathrm{CDCl}_{3}\right)$. 


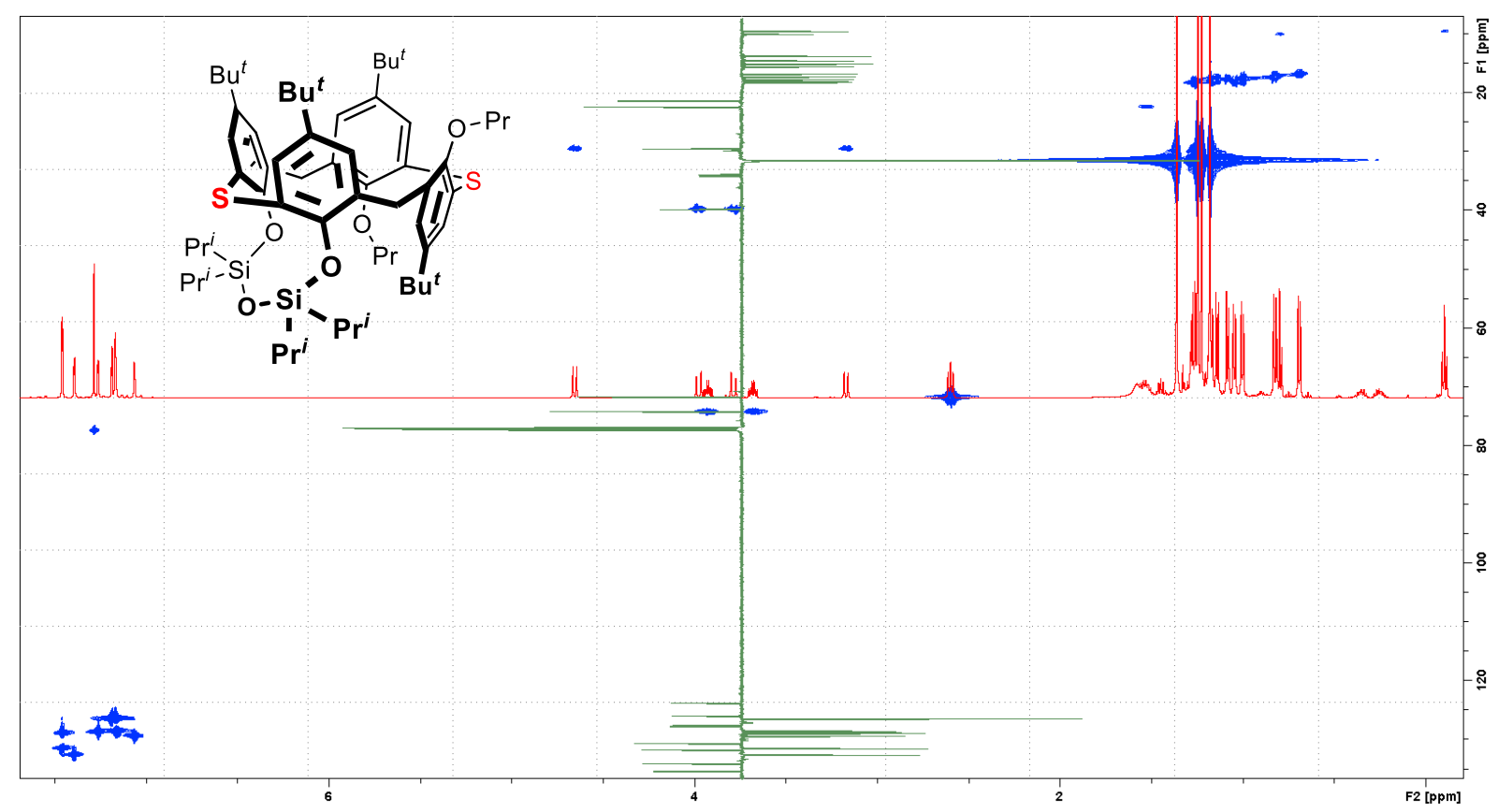

Figure S32. HMQC NMR of compound $4\left(\mathrm{CDCl}_{3}\right)$.

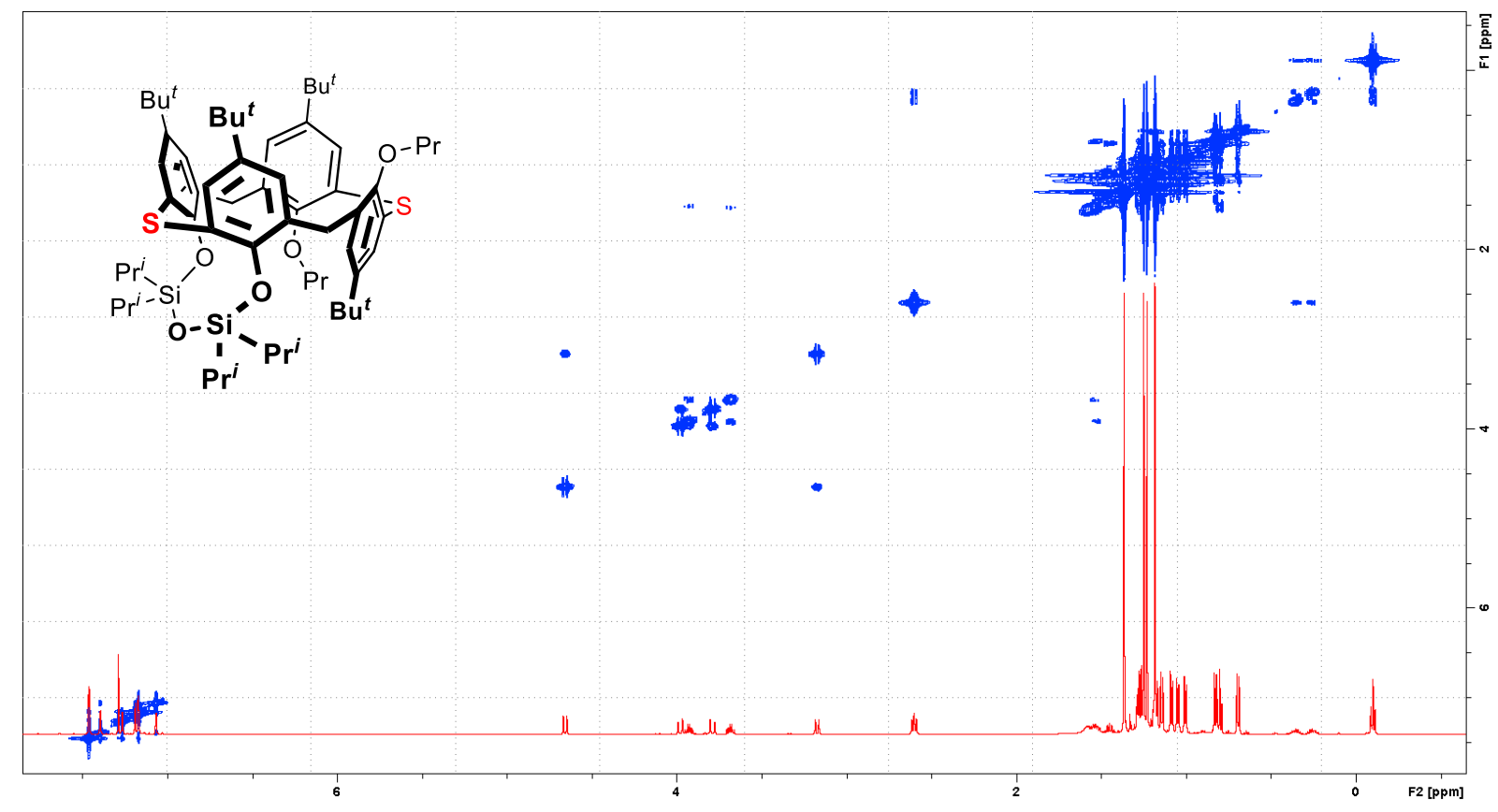

Figure S33. COSY NMR of compound $4\left(\mathrm{CDCl}_{3}\right)$. 
145 Kortus_ESIpos_DK77_F4_1

4/18/2019 9:25:33 AM

145 Kortus ESIpos DK77 F4 1 \#48-50 RT: 0.69-0.72 AV: 3 NL: $6.12 E 7$

T: FTMS + C ESI Full ms [200.00-2000.00]

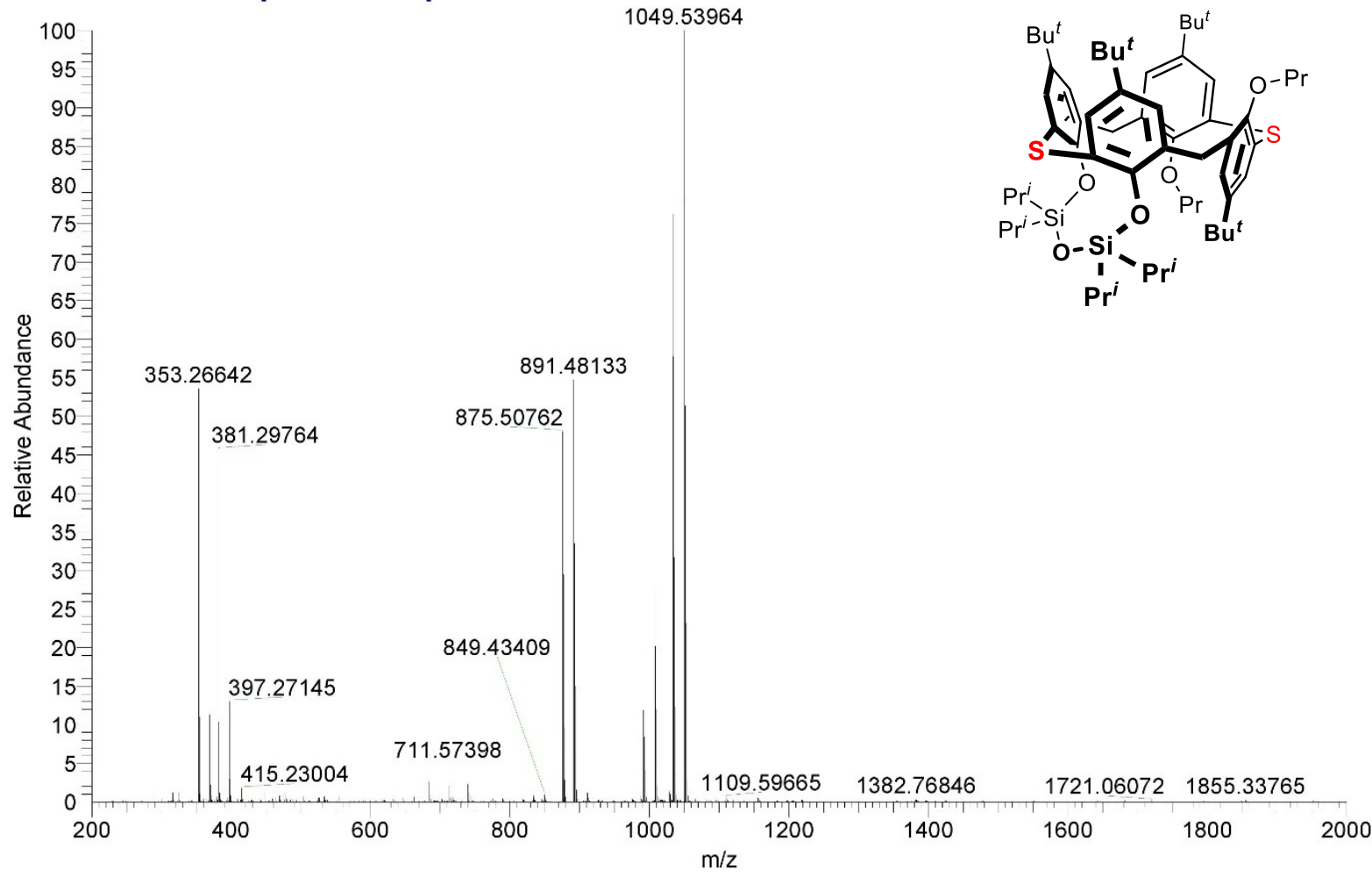

Figure S34. HRMS of compound $4\left(\mathrm{ESI}^{+}\right)$.

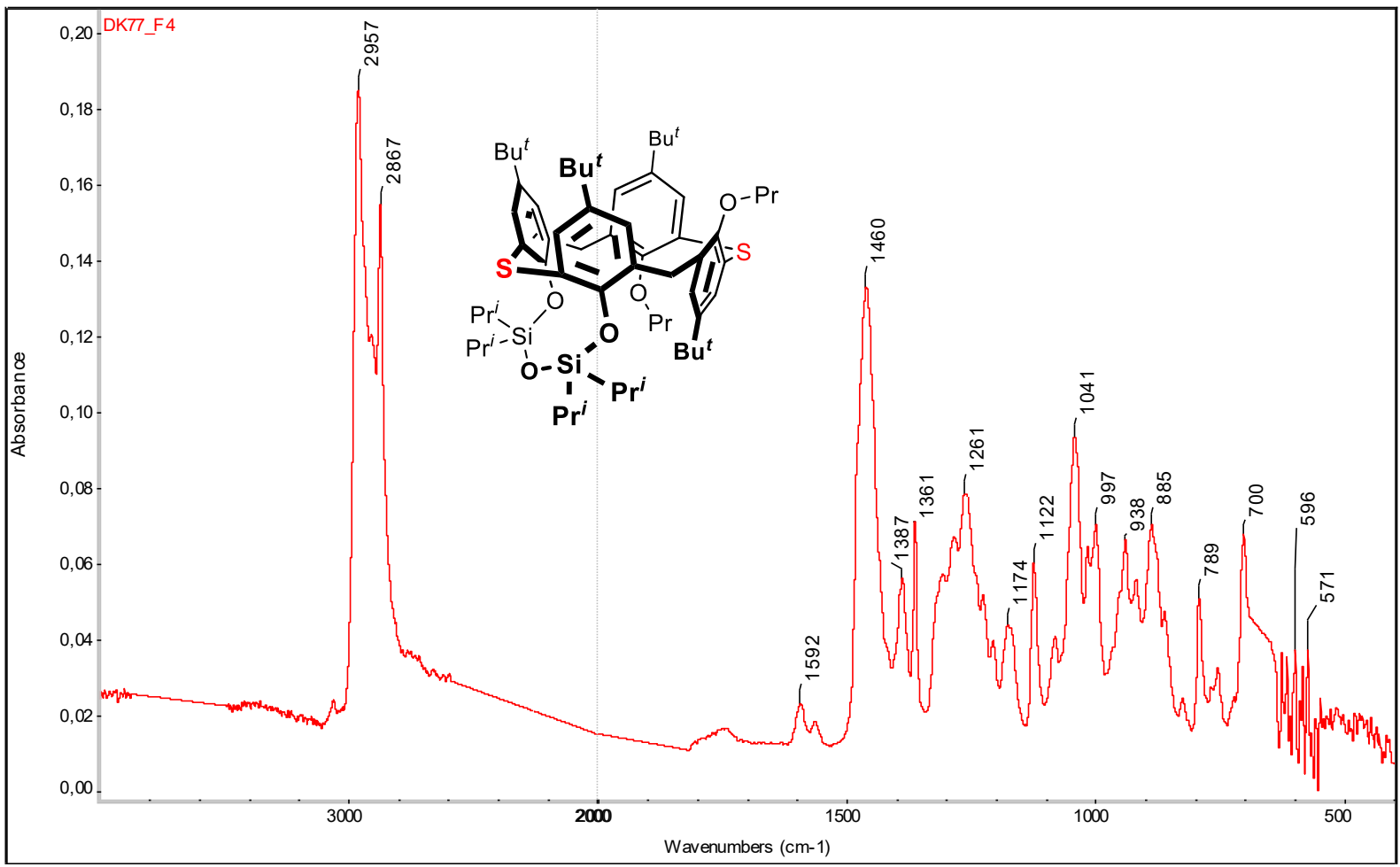

Figure S35. IR of compound 4 (ATR). 


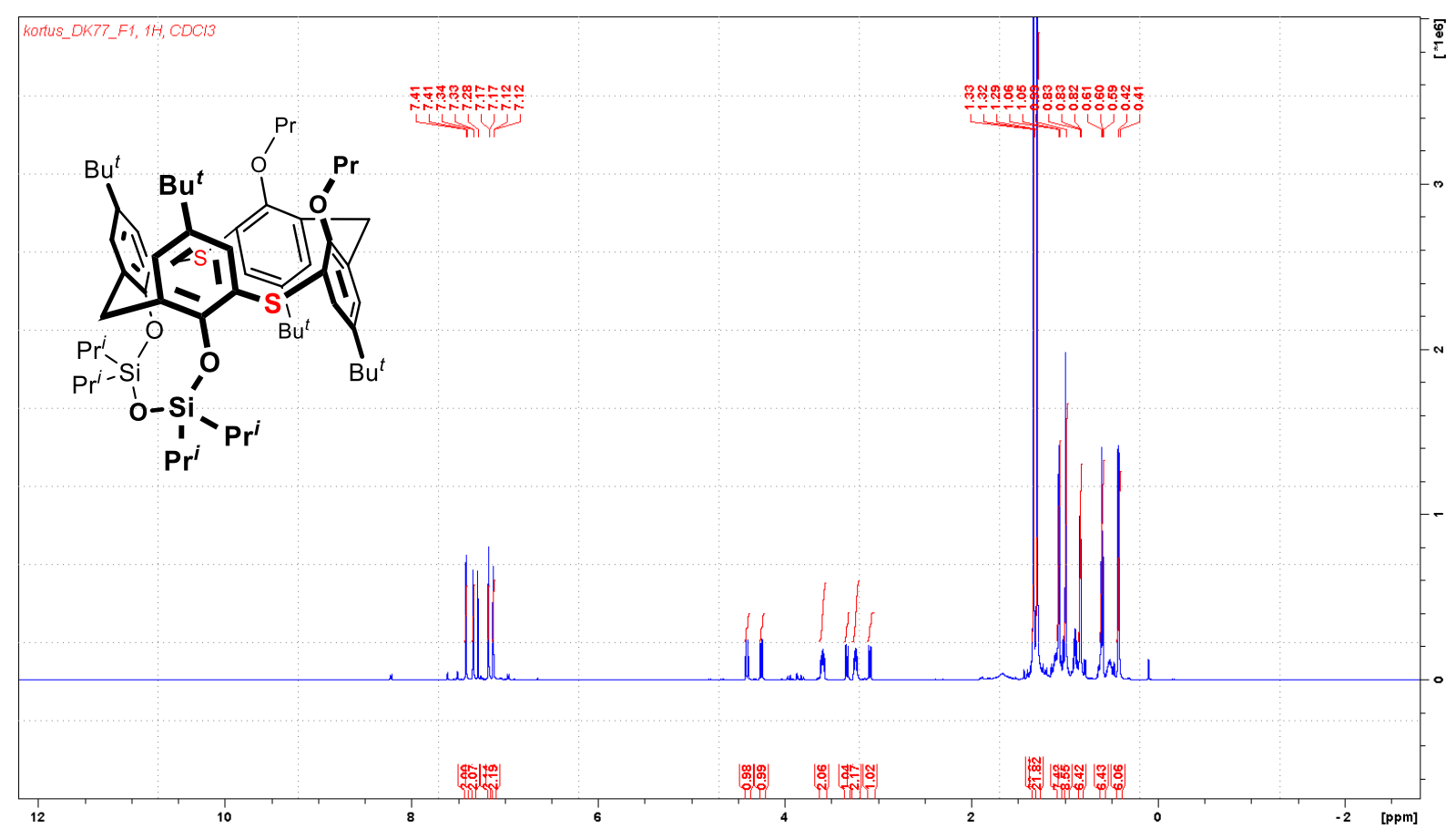

Figure S36. ${ }^{1} \mathrm{H} \mathrm{NMR}$ of compound $5\left(\mathrm{CDCl}_{3}, 600 \mathrm{MHz}\right)$.

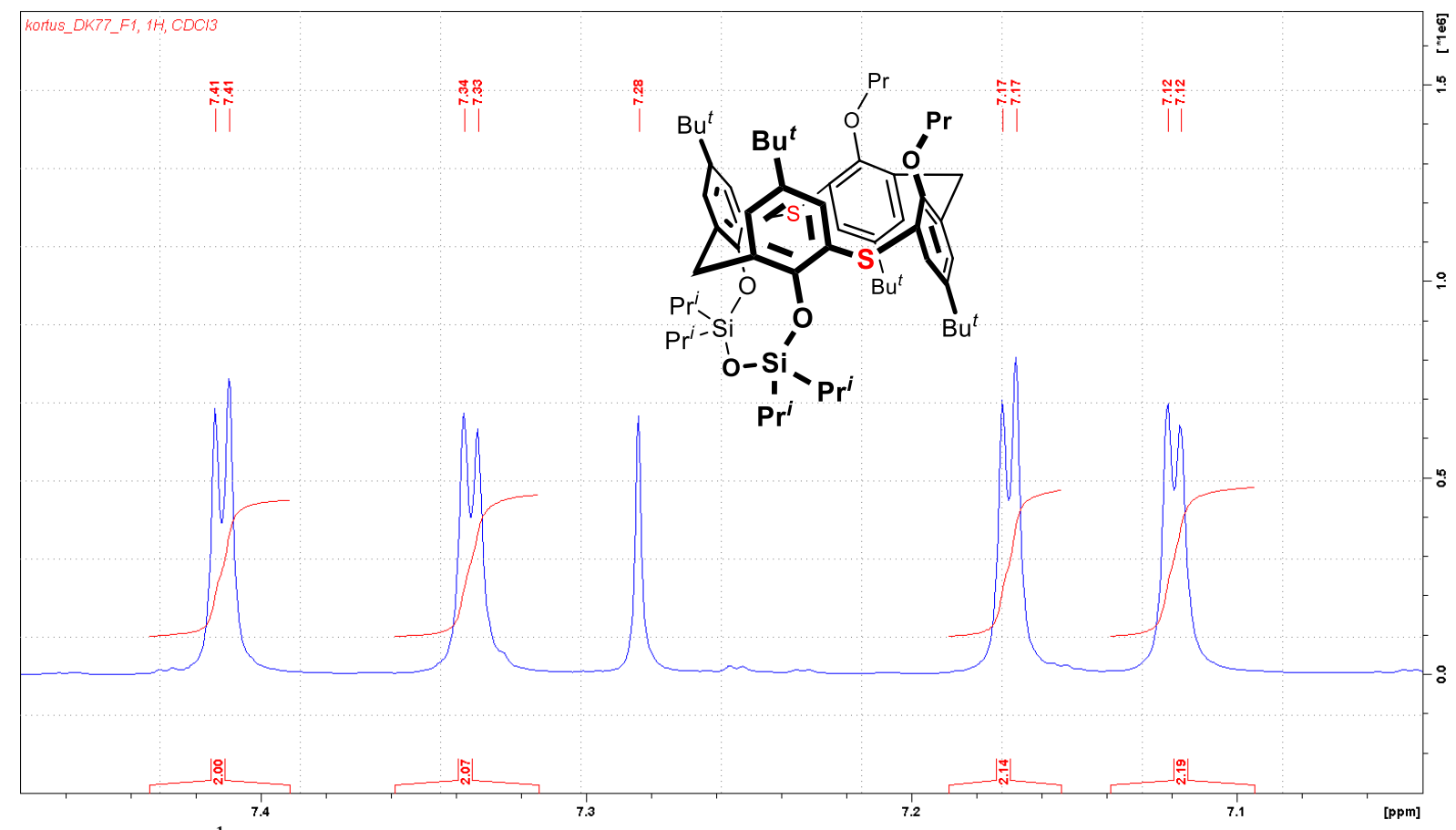

Figure S37. ${ }^{1} \mathrm{H}$ NMR of compound 5, aromatic region $\left(\mathrm{CDCl}_{3}, 600 \mathrm{MHz}\right)$. 


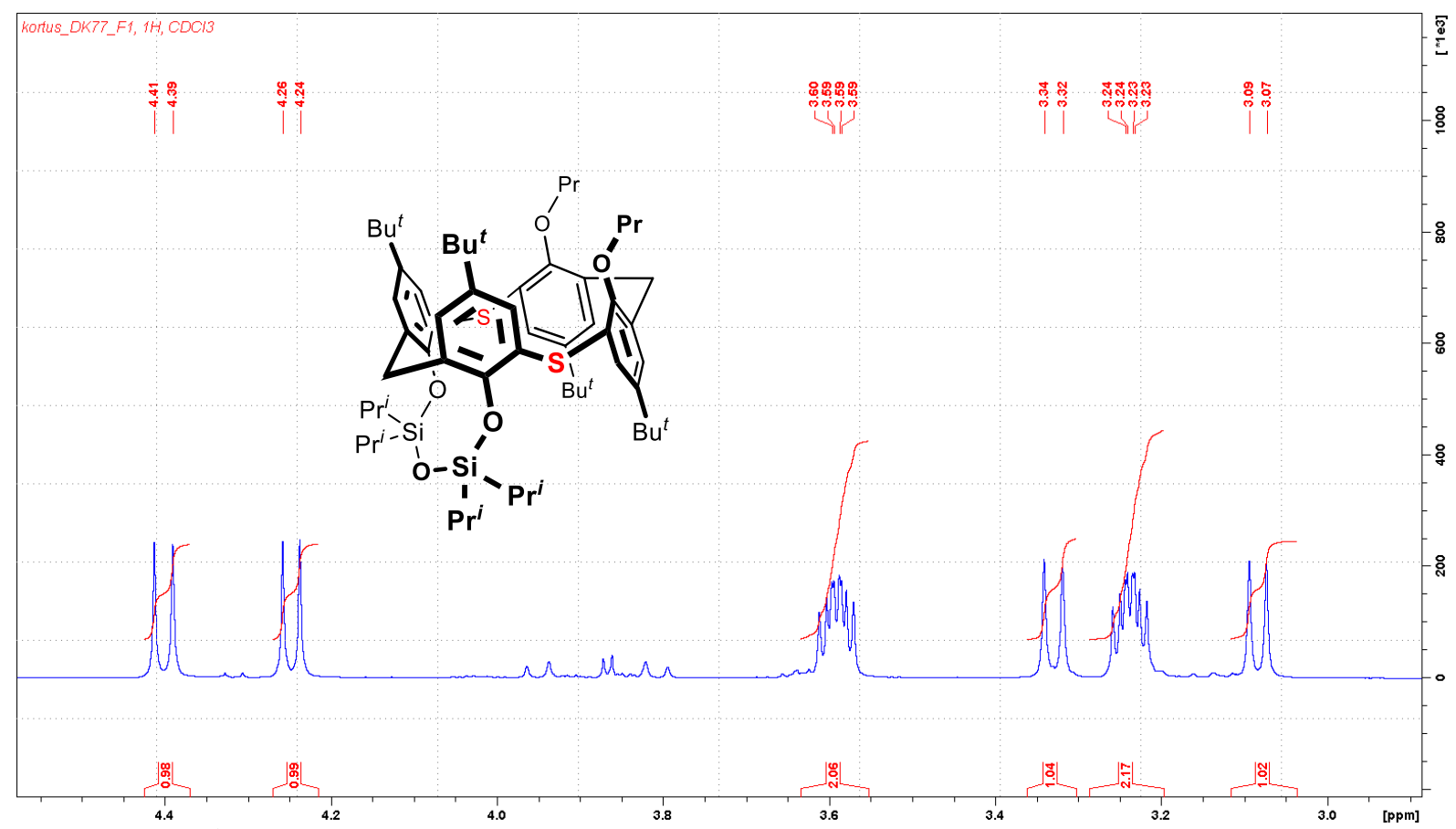

Figure S38. ${ }^{1} \mathrm{H}$ NMR of compound 5, bridge region $\left(\mathrm{CDCl}_{3}, 600 \mathrm{MHz}\right)$.

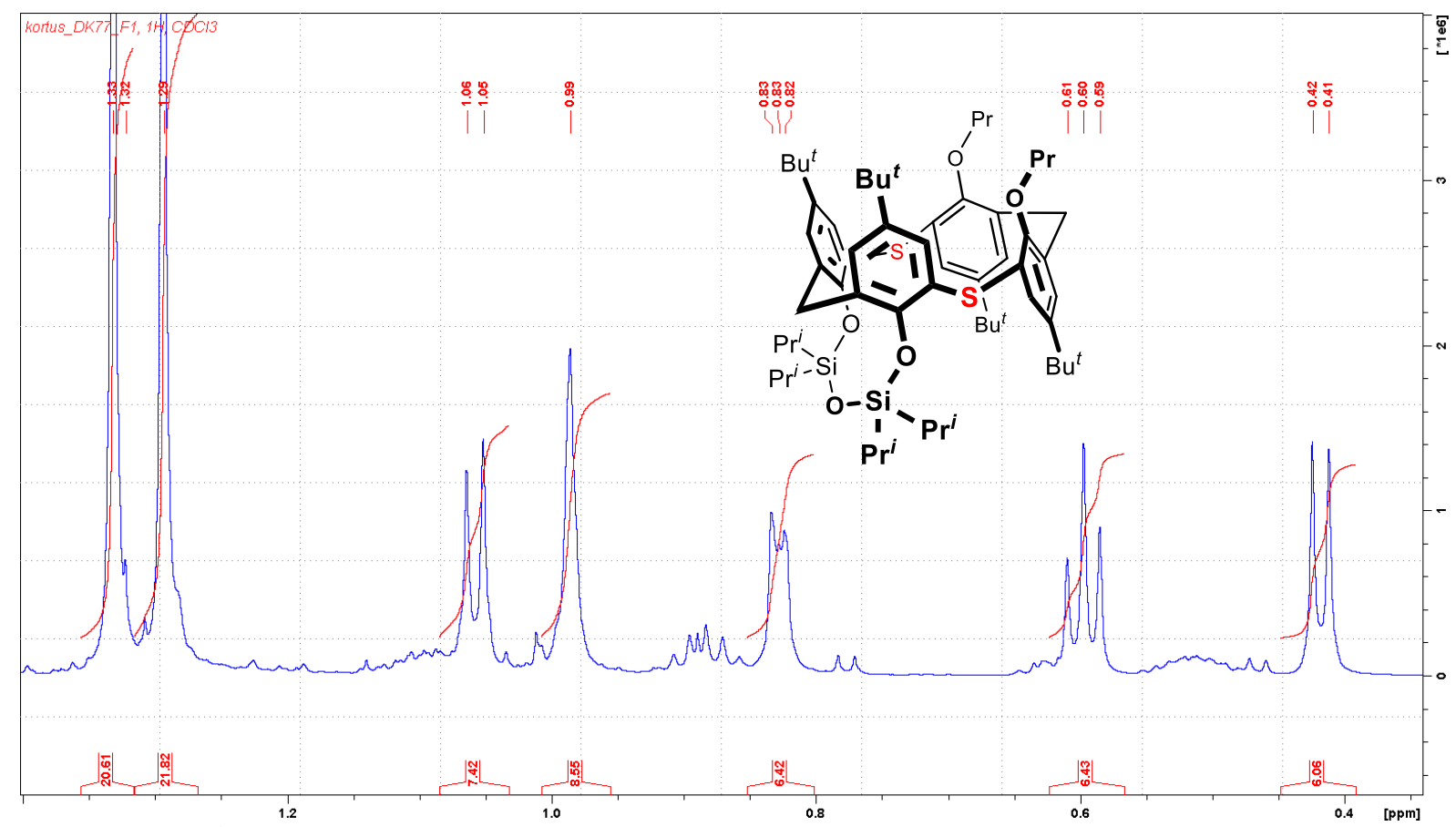

Figure S39. ${ }^{1} \mathrm{H}$ NMR of compound 5, aliphatic region $\left(\mathrm{CDCl}_{3}, 600 \mathrm{MHz}\right)$. 


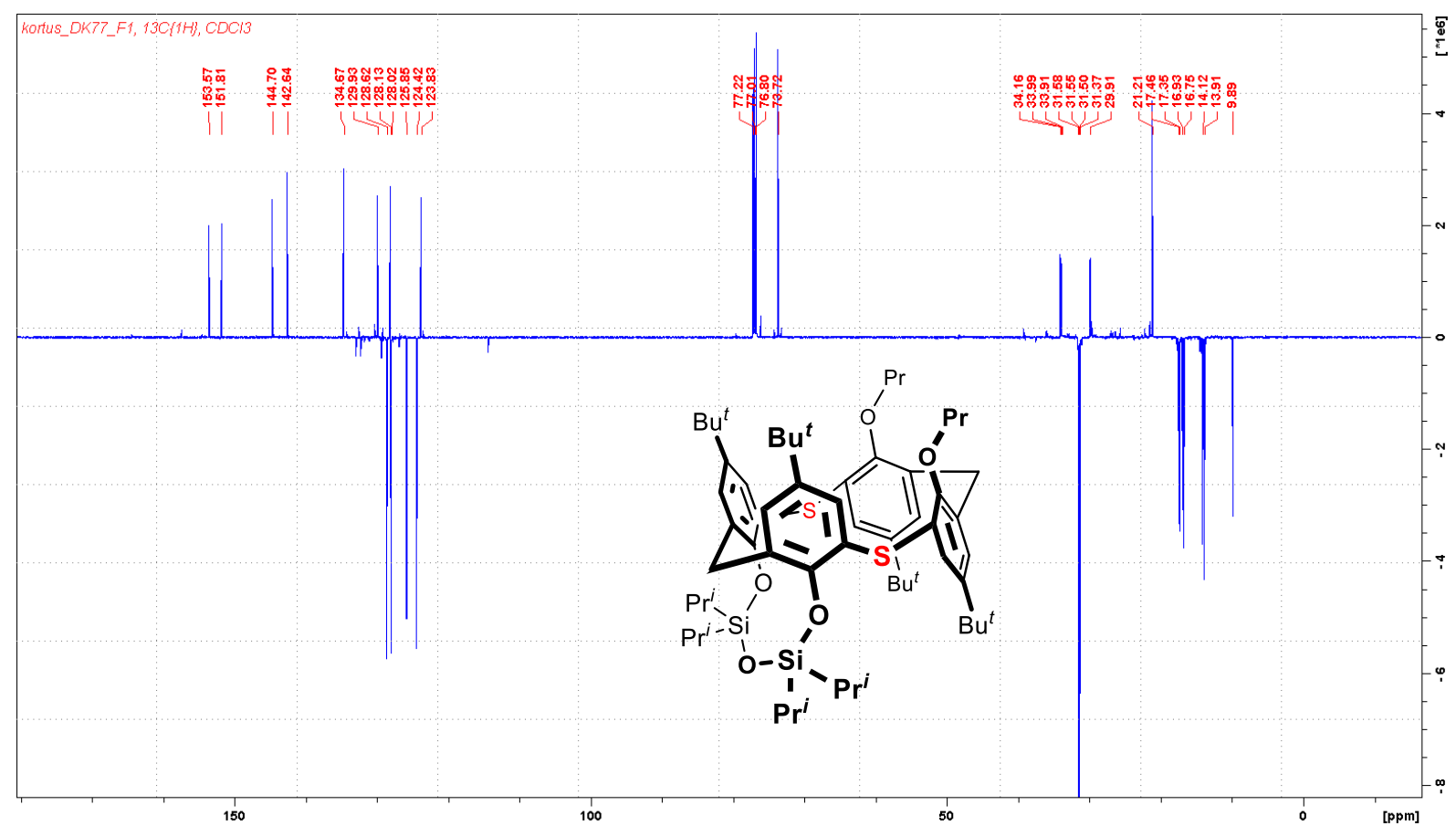

Figure S40. ${ }^{13} \mathrm{C}\left\{{ }^{1} \mathrm{H}\right\} \mathrm{NMR}$ of compound $\mathbf{5}\left(\mathrm{CDCl}_{3}, 151 \mathrm{MHz}\right)$.

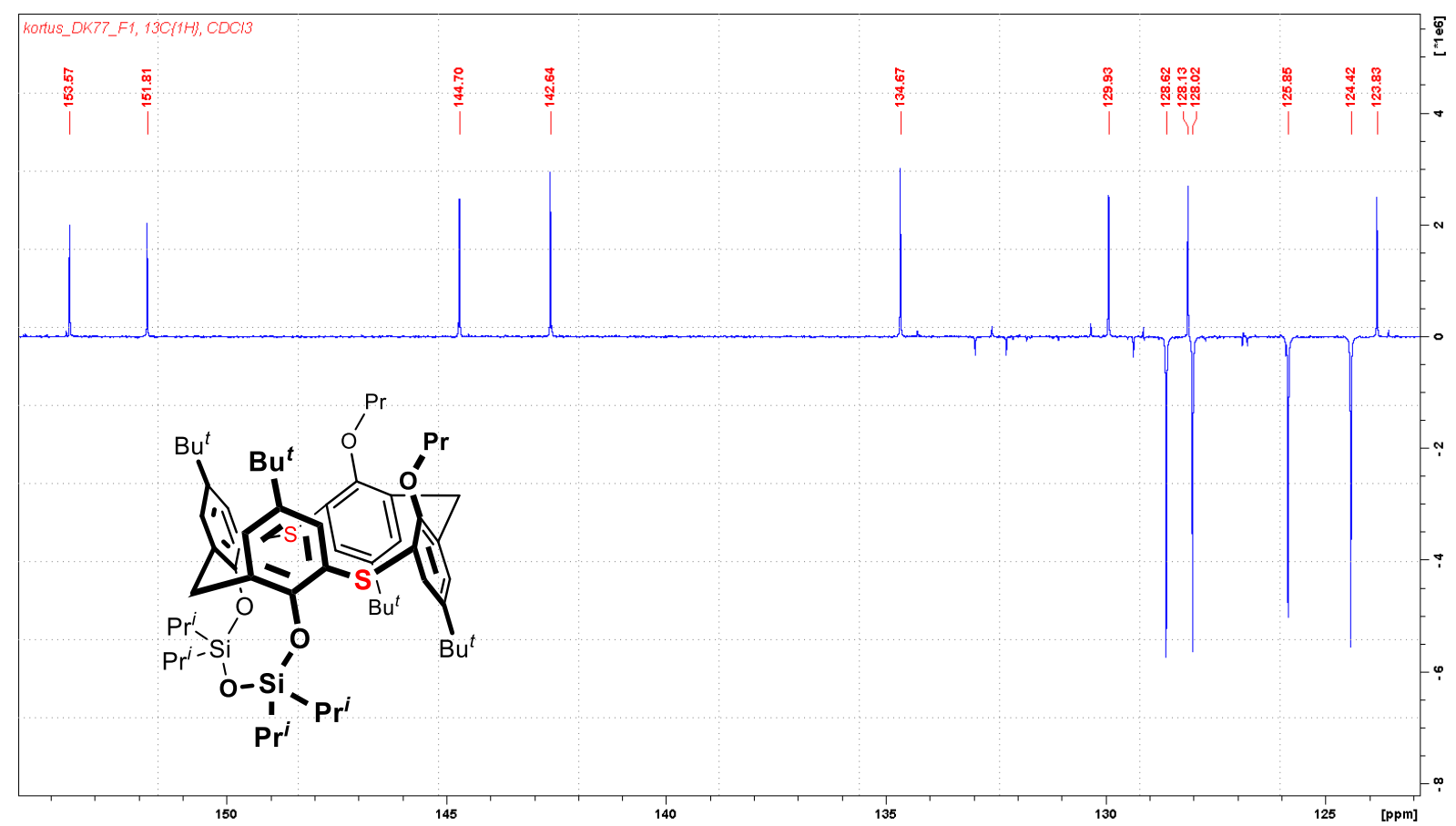

Figure S41. ${ }^{13} \mathrm{C}\left\{{ }^{1} \mathrm{H}\right\}$ NMR of compound 5, aromatic region $\left(\mathrm{CDCl}_{3}, 151 \mathrm{MHz}\right)$. 


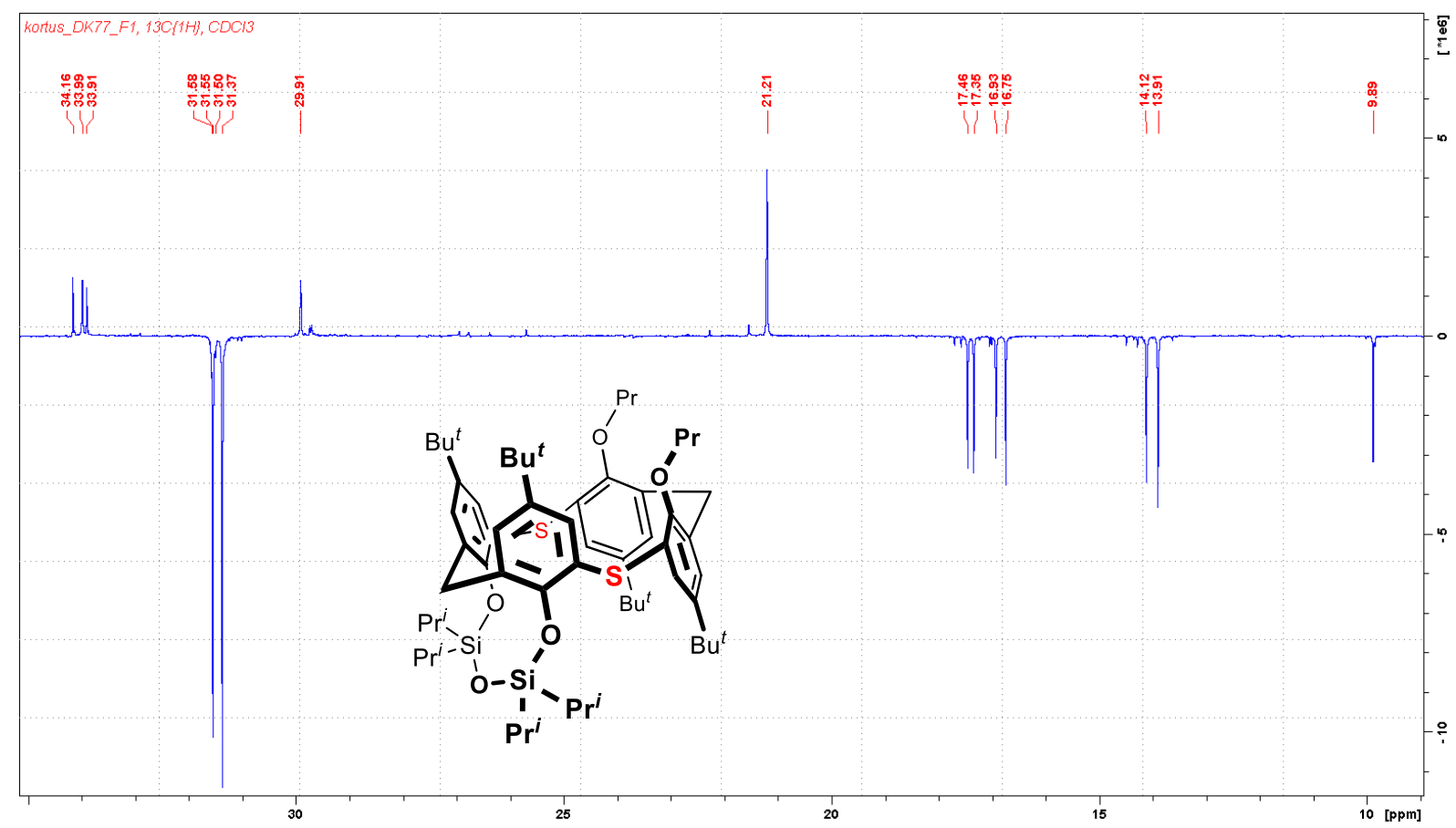

Figure S42. ${ }^{13} \mathrm{C}\left\{{ }^{1} \mathrm{H}\right\}$ NMR of compound 5, aliphatic region $\left(\mathrm{CDCl}_{3}, 151 \mathrm{MHz}\right)$.

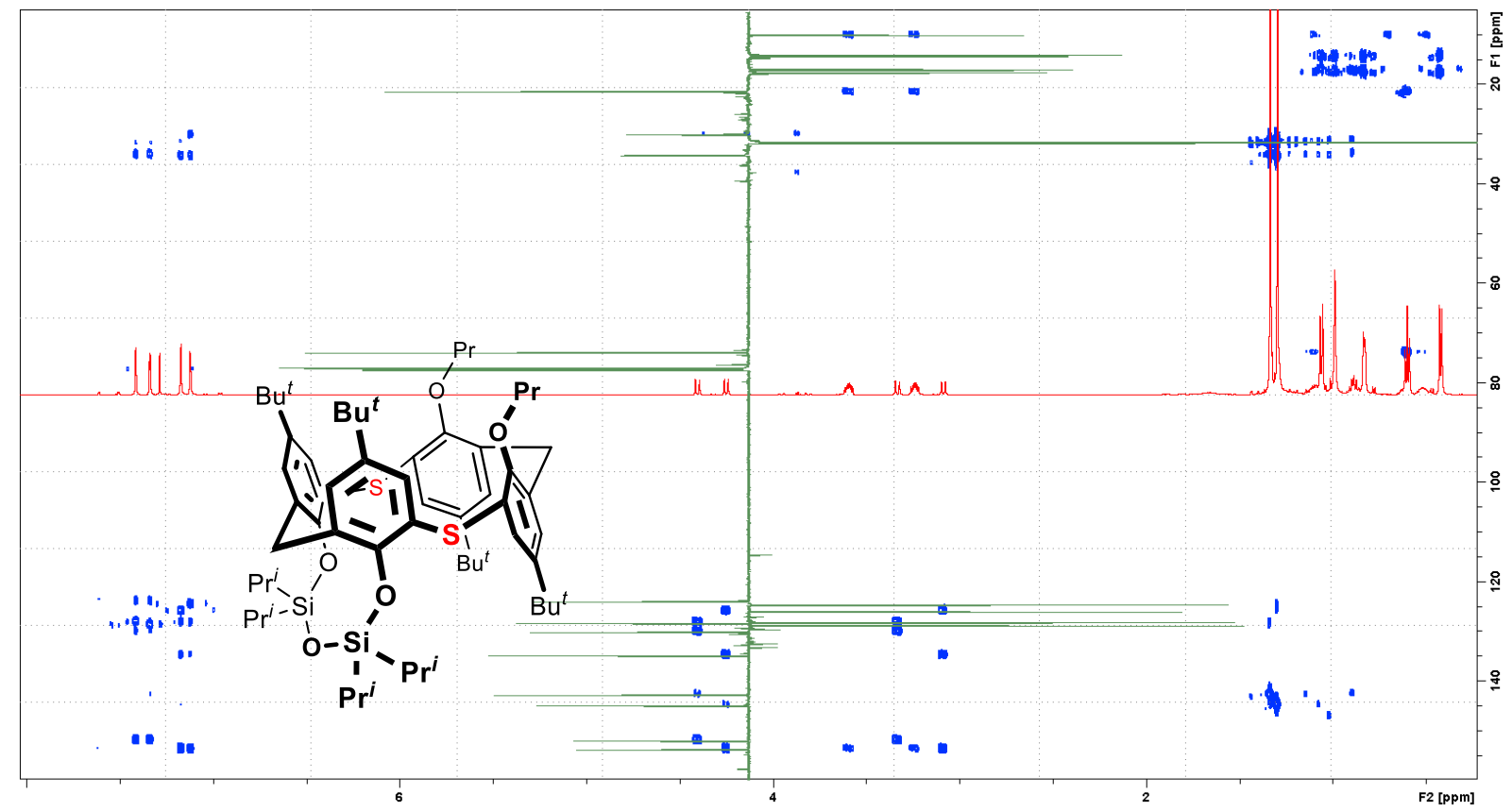

Figure S43. HMBC NMR of compound $5\left(\mathrm{CDCl}_{3}\right)$. 


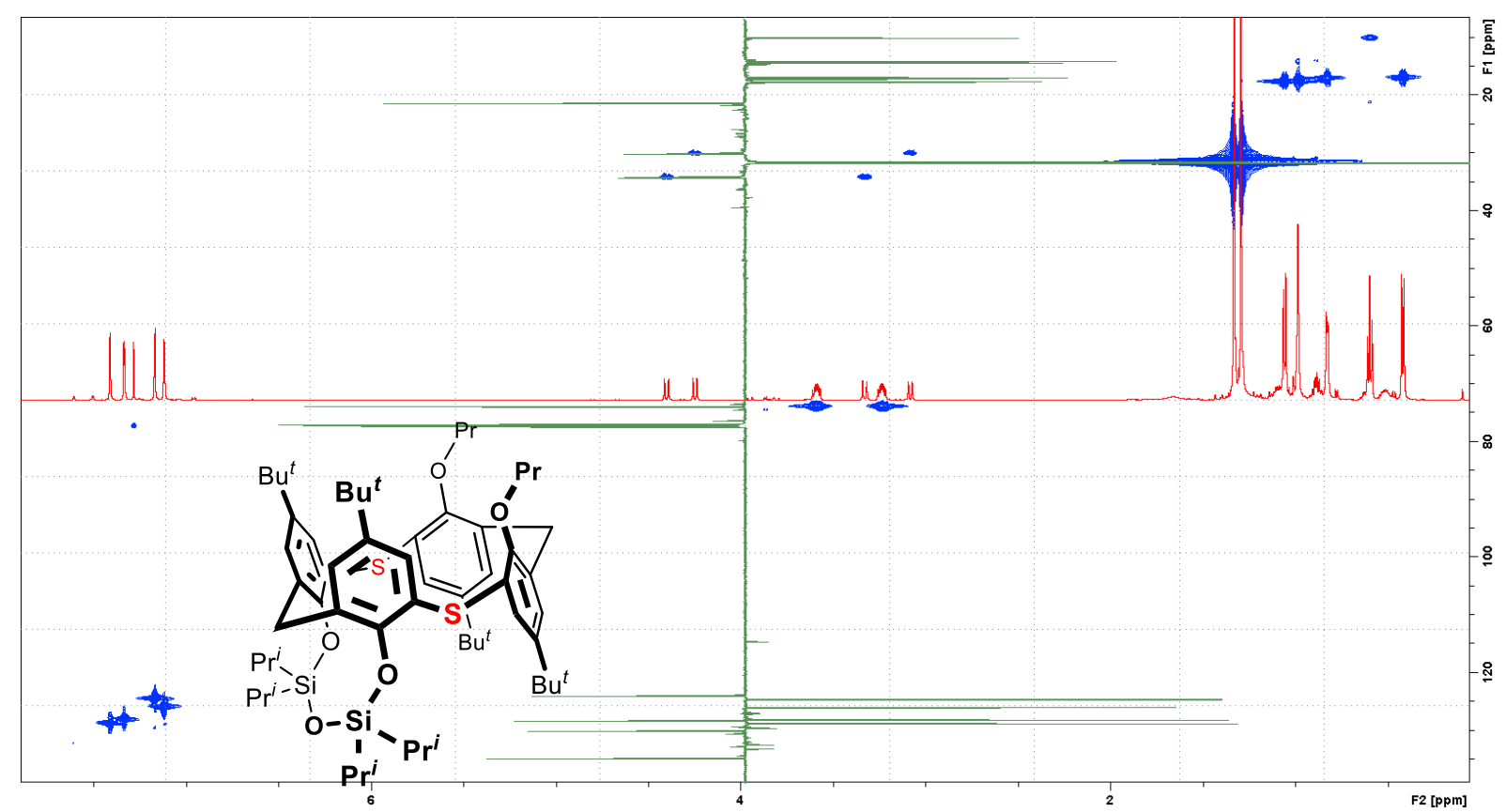

Figure S44. HMQC NMR of compound $5\left(\mathrm{CDCl}_{3}\right)$.

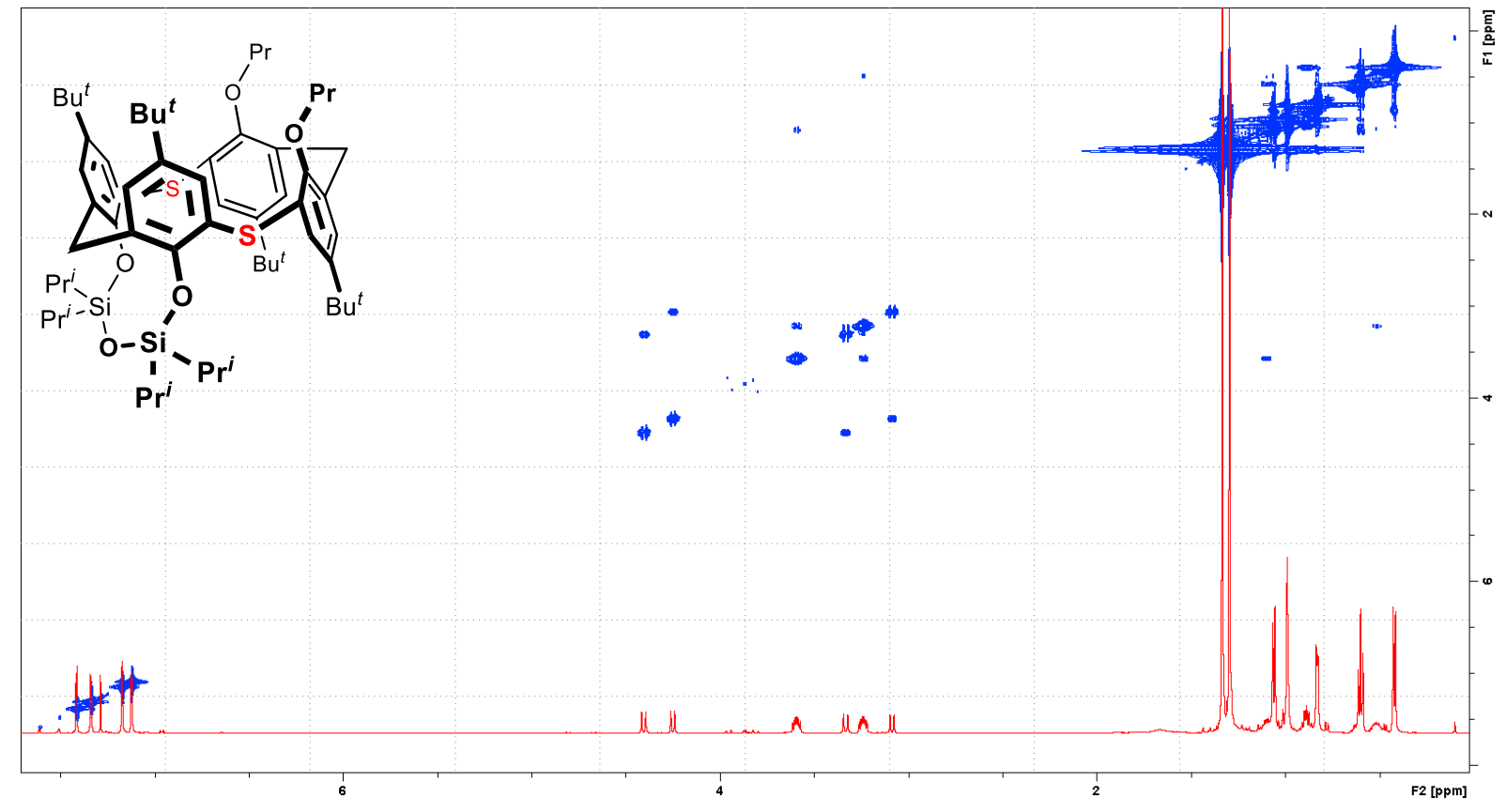

Figure S45. COSY NMR of compound $5\left(\mathrm{CDCl}_{3}\right)$. 
145 Kortus_ESIpos_DK77_F1_2

$\mathrm{MeOH}$

145_Kortus ESIpos DK77 F1 2 \#17-22 RT: 0.24-0.31 AV: 6 NL: $1.81 \mathrm{E} 7$

T: FTMS + c ESI Full ms [200.00-2000.00]

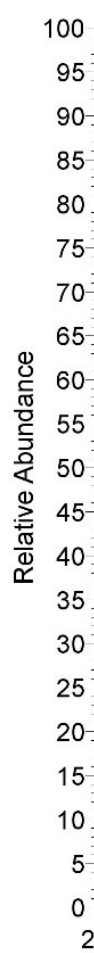
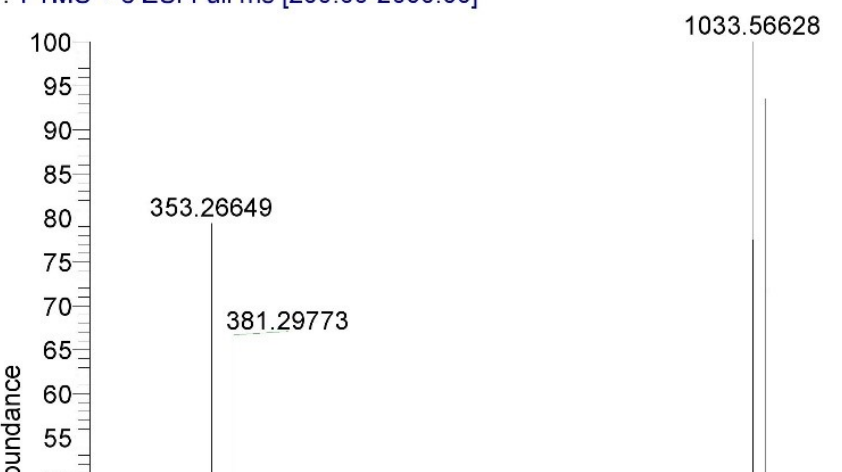

53.26649

$75^{-}$
$70^{\prime}$
$65^{-}$
$60^{-}$
$55^{-}$

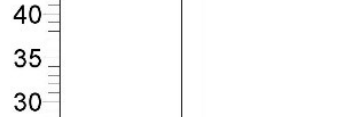

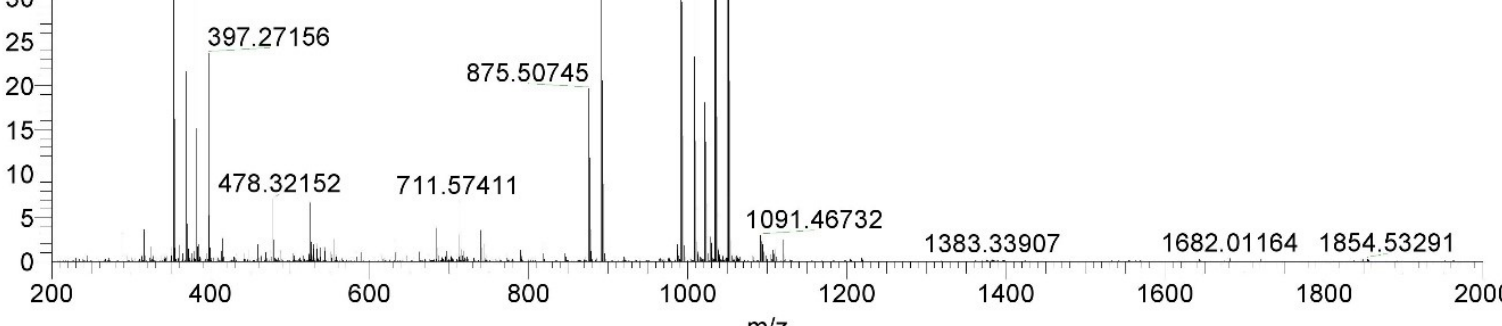

Figure S46. HRMS of compound $5\left(\mathrm{ESI}^{+}\right)$.

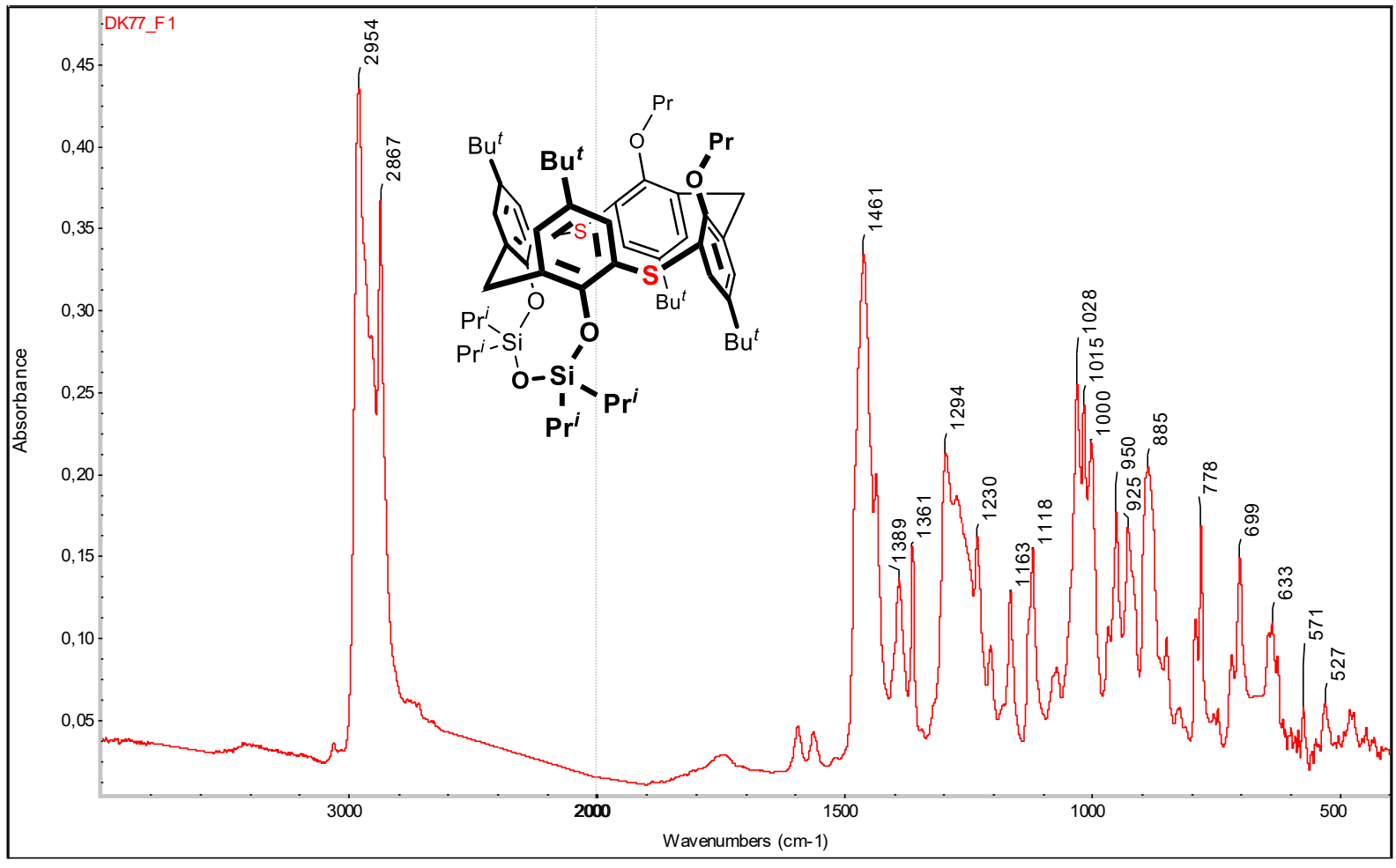

Figure S47. IR of compound 5 (ATR). 


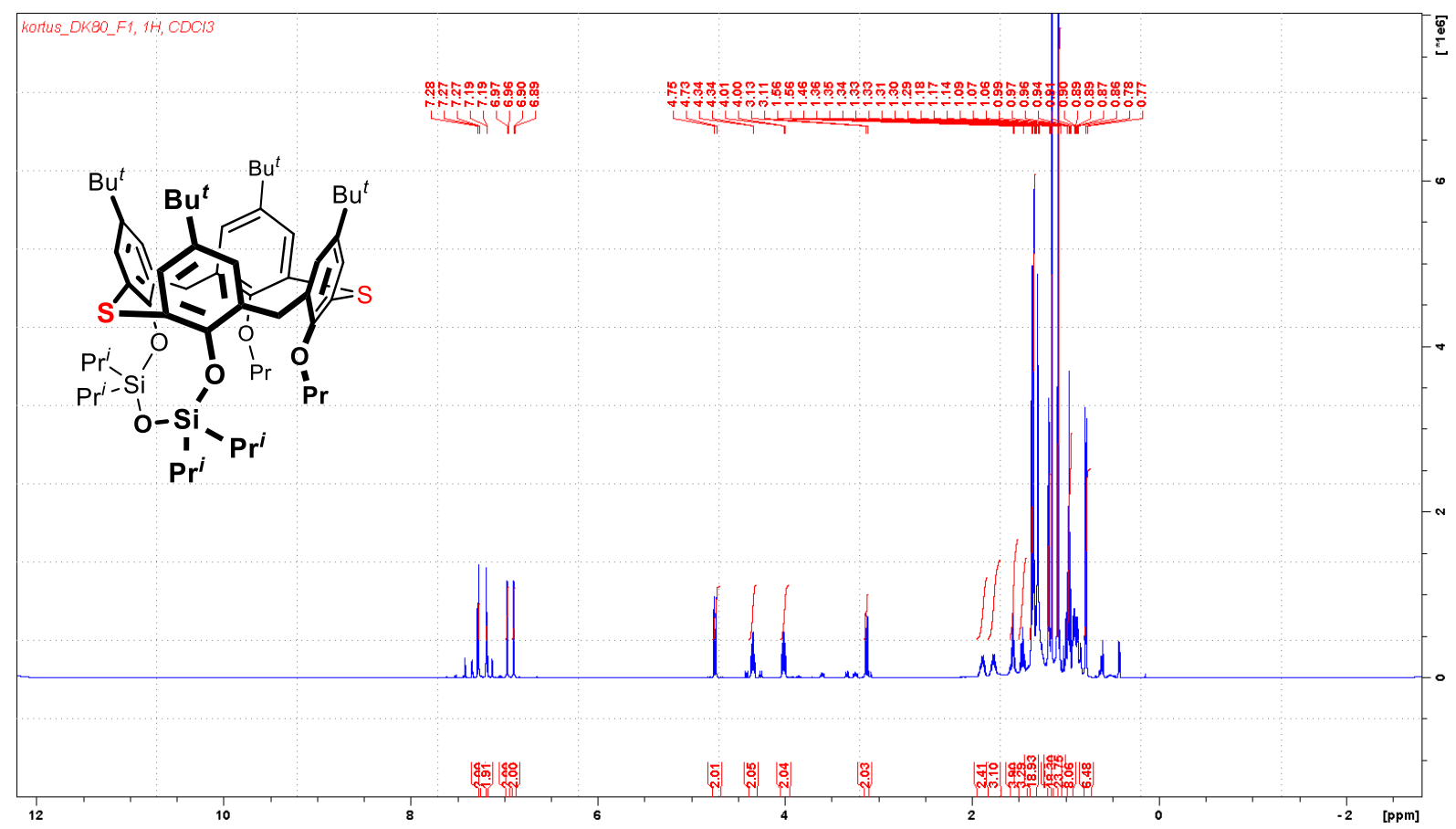

Figure S48. ${ }^{1} \mathrm{H} \mathrm{NMR}$ of compound $6\left(\mathrm{CDCl}_{3}, 600 \mathrm{MHz}\right)$.

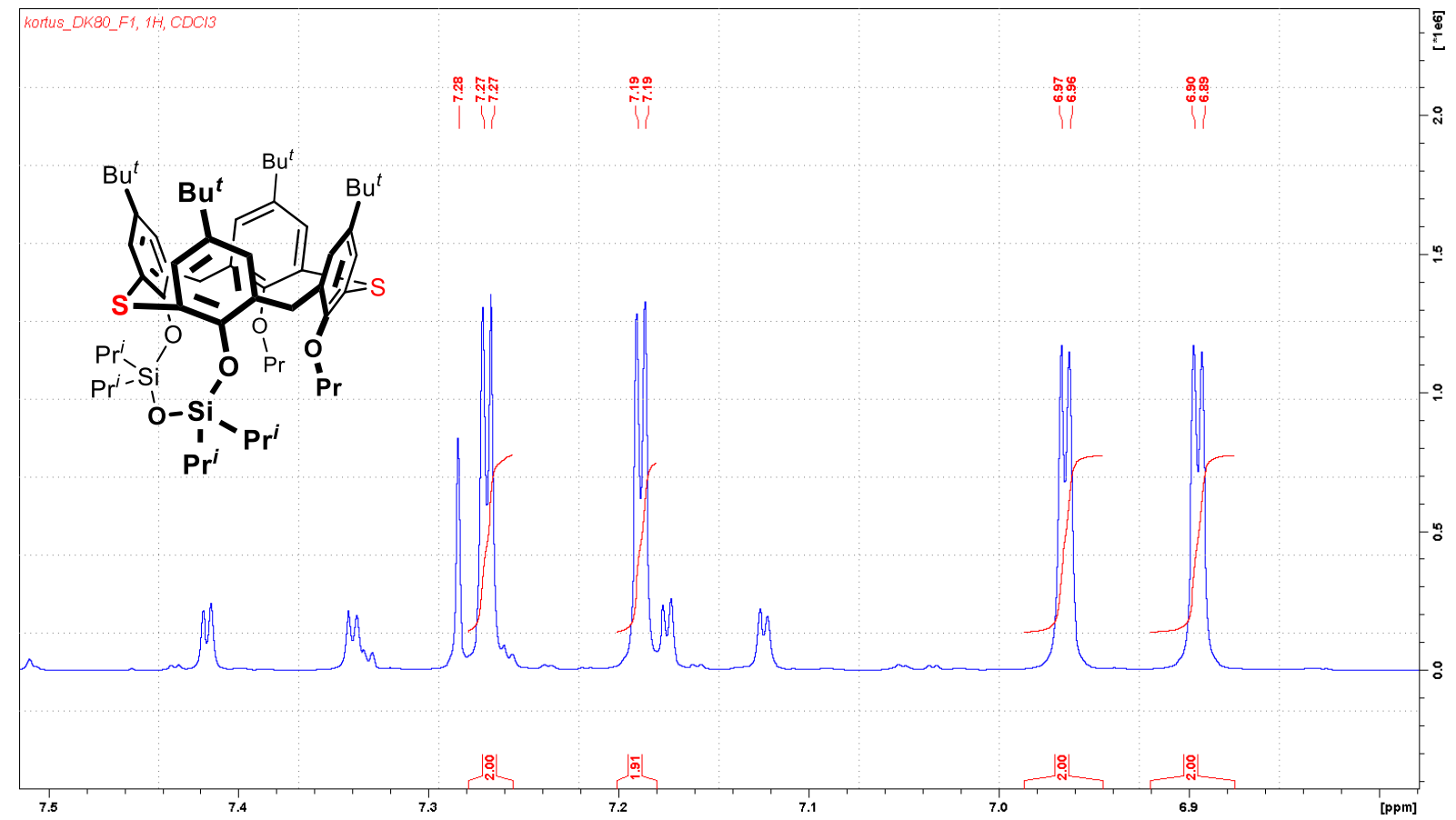

Figure S49. ${ }^{1} \mathrm{H}$ NMR of compound 6, aromatic region $\left(\mathrm{CDCl}_{3}, 600 \mathrm{MHz}\right)$. 


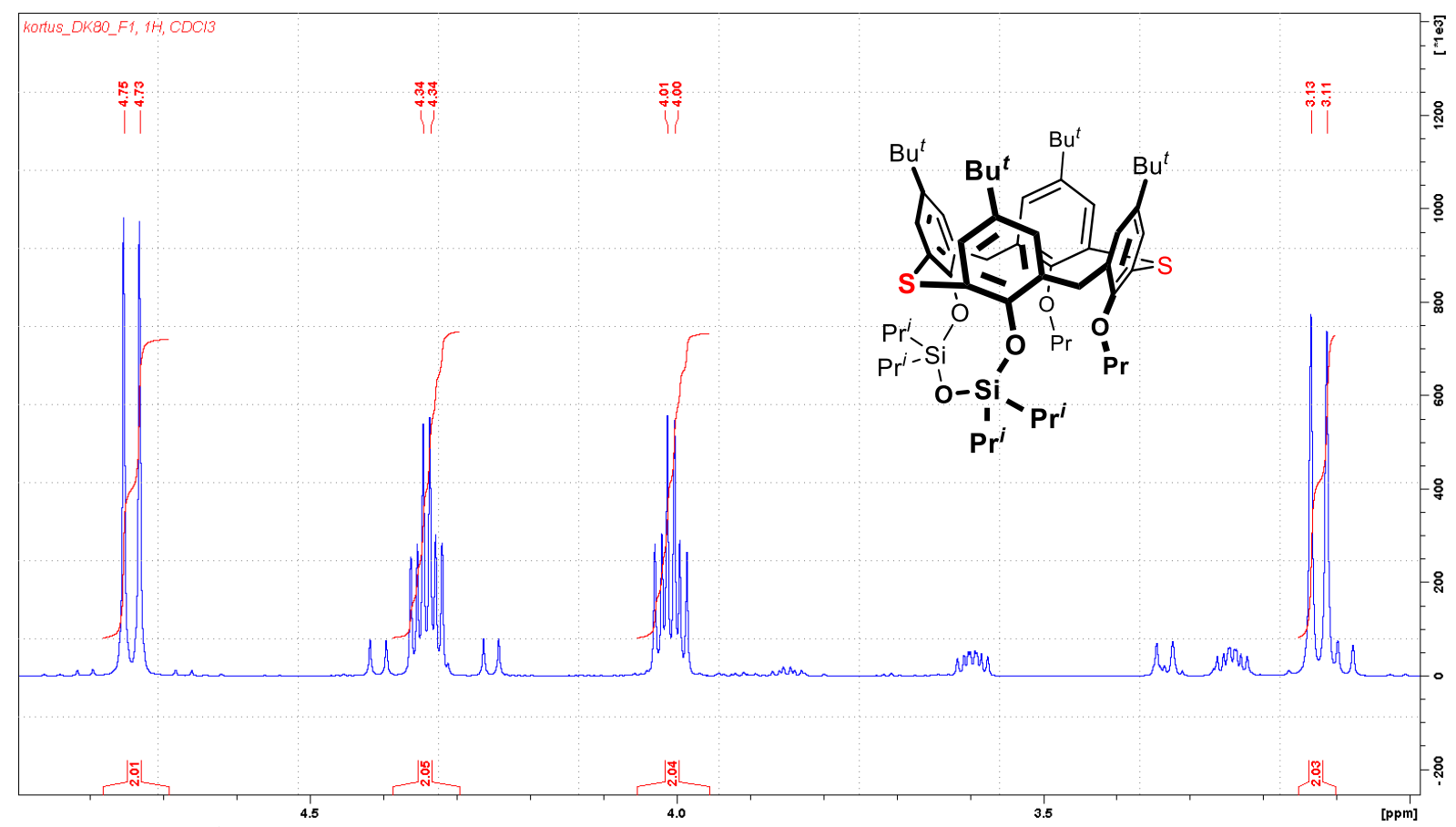

Figure S50. ${ }^{1} \mathrm{H}$ NMR of compound 6, bridge region $\left(\mathrm{CDCl}_{3}, 600 \mathrm{MHz}\right)$.

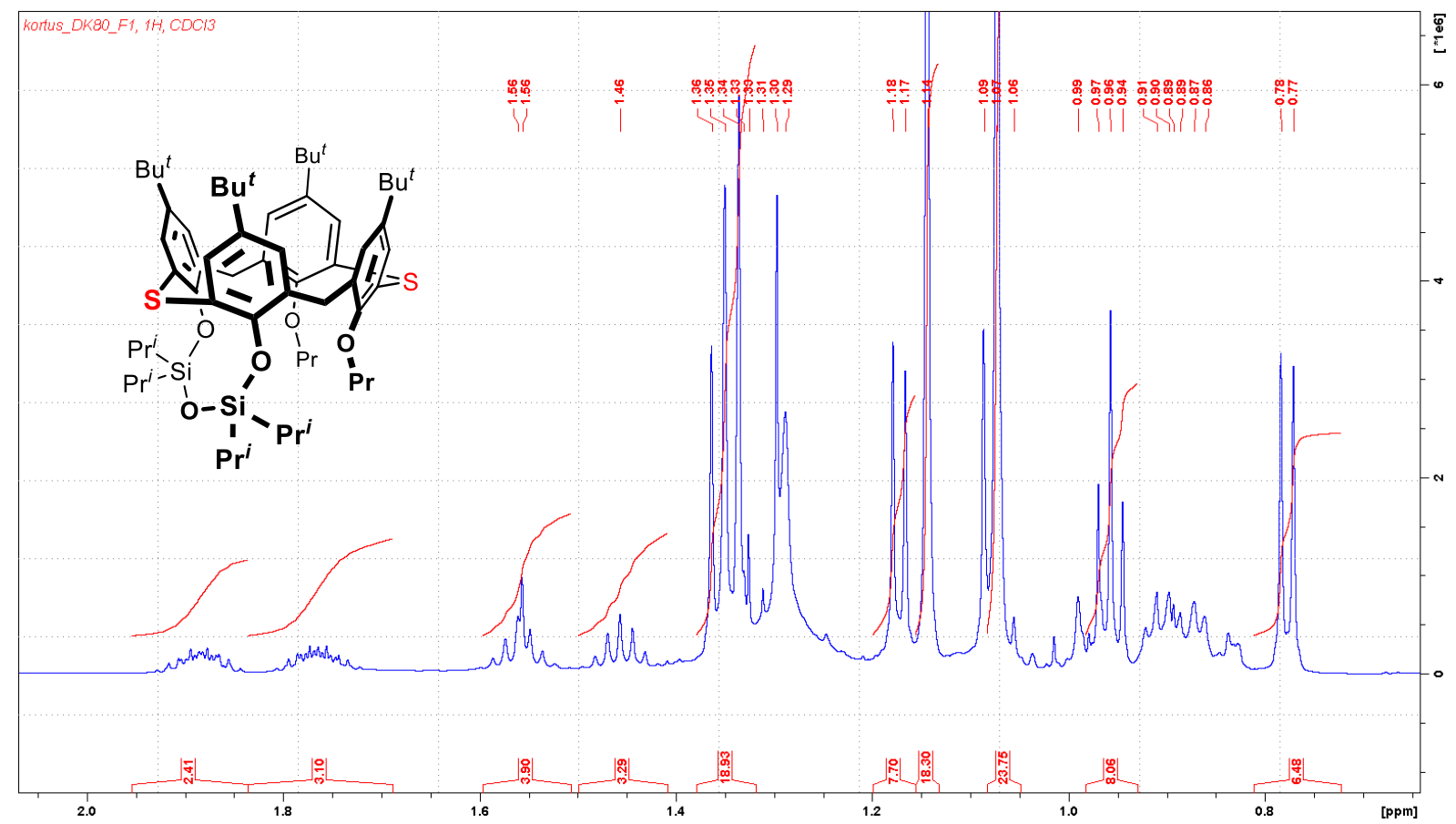

Figure S51. ${ }^{1} \mathrm{H}$ NMR of compound 6, aliphatic region $\left(\mathrm{CDCl}_{3}, 600 \mathrm{MHz}\right)$. 


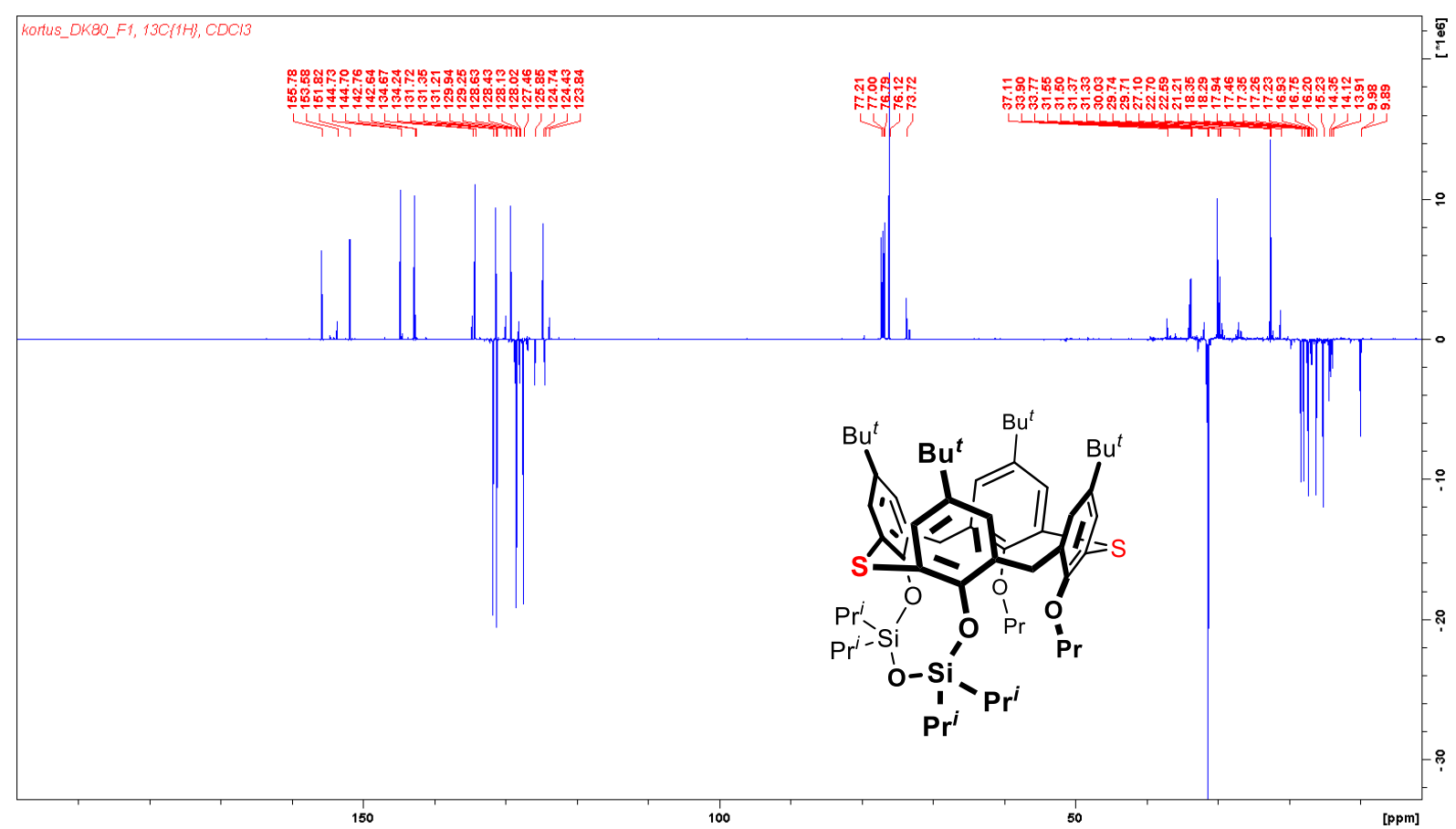

Figure S52. ${ }^{13} \mathrm{C}\left\{{ }^{1} \mathrm{H}\right\}$ NMR of compound $6\left(\mathrm{CDCl}_{3}, 151 \mathrm{MHz}\right)$.

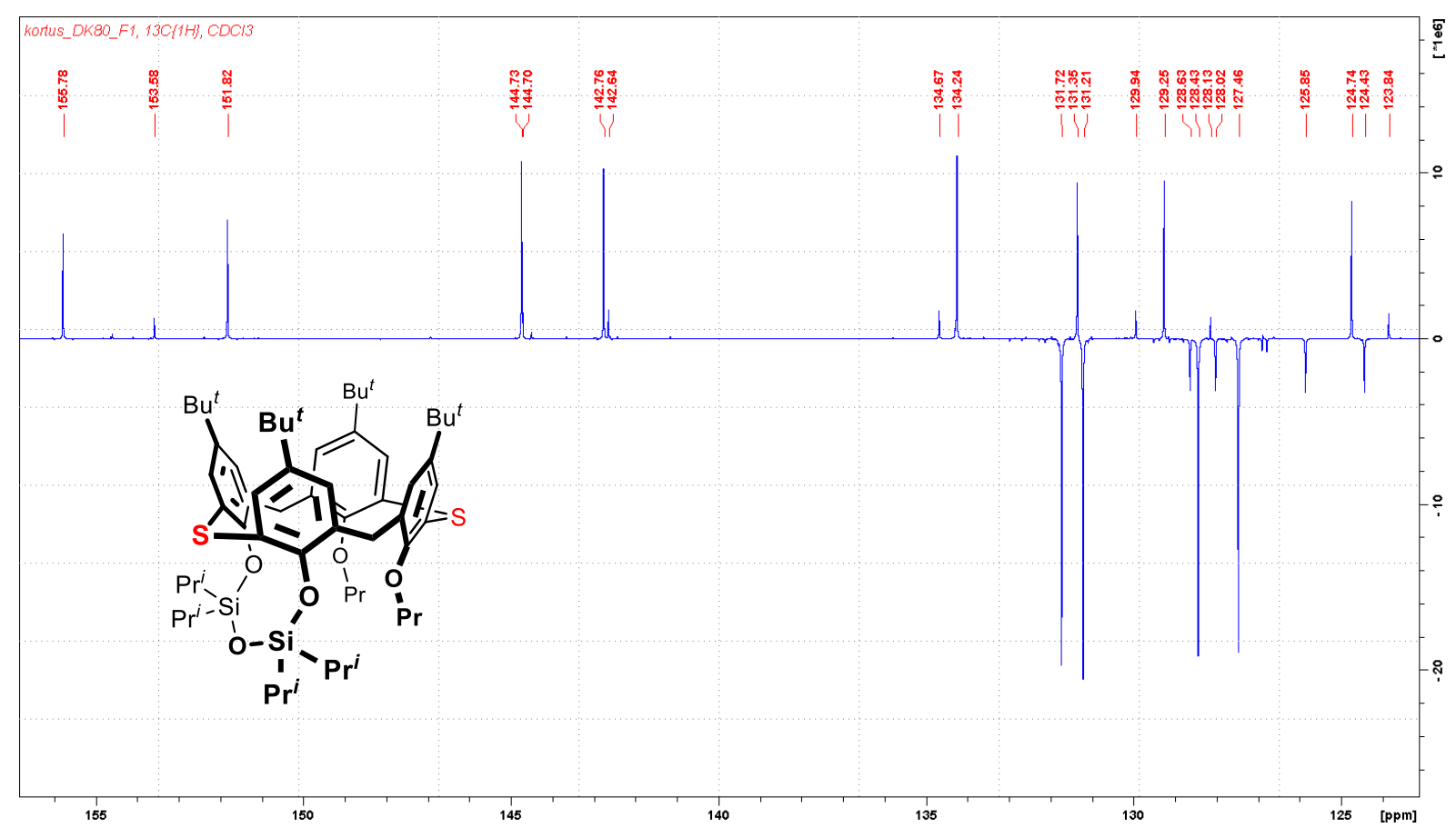

Figure S53. ${ }^{13} \mathrm{C}\left\{{ }^{1} \mathrm{H}\right\}$ NMR of compound 6, aromatic region $\left(\mathrm{CDCl}_{3}, 151 \mathrm{MHz}\right)$. 


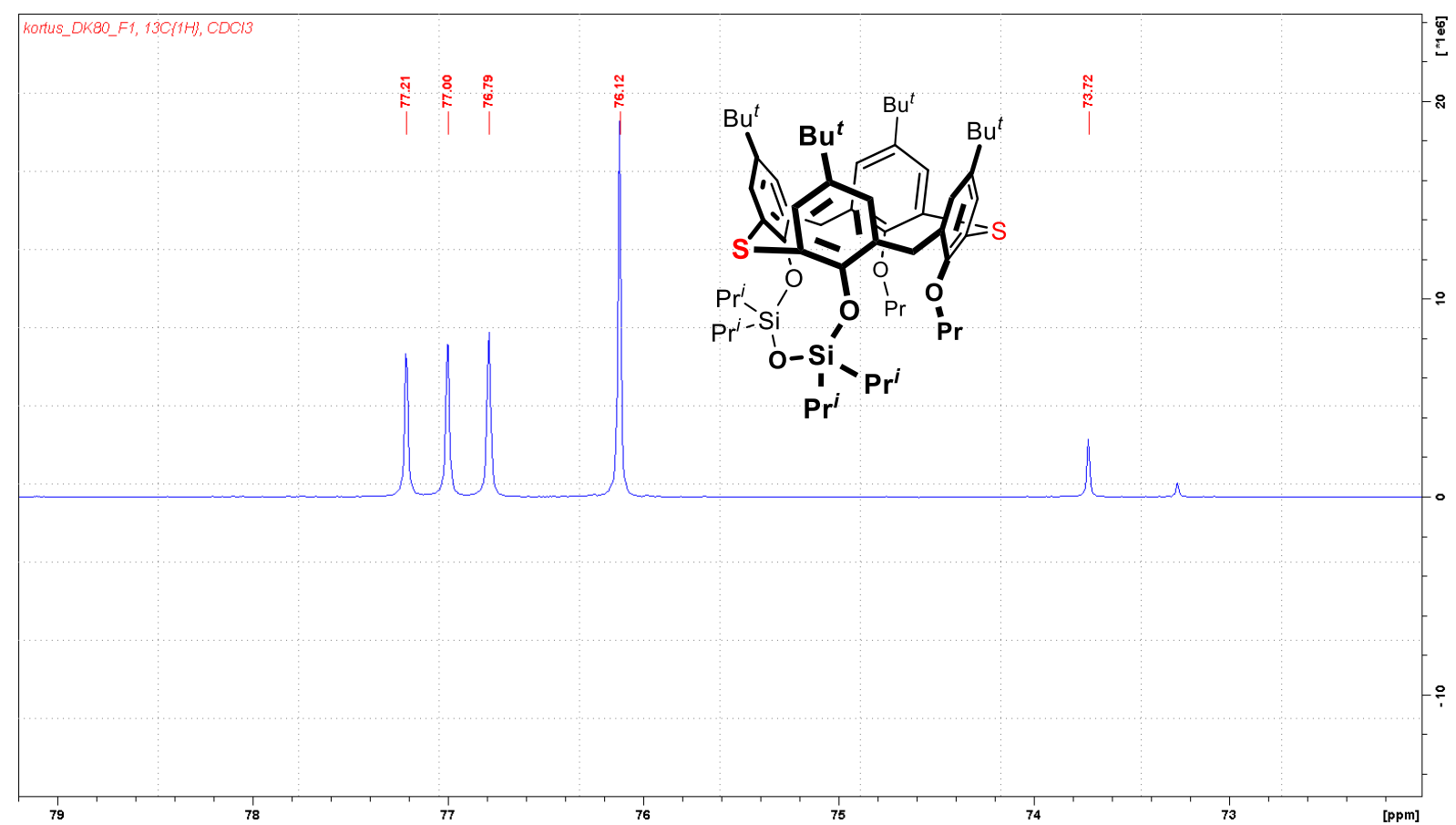

Figure S54. ${ }^{13} \mathrm{C}\left\{{ }^{1} \mathrm{H}\right\}$ NMR of compound 6, aliphatic region $\left(\mathrm{CDCl}_{3}, 151 \mathrm{MHz}\right)$.

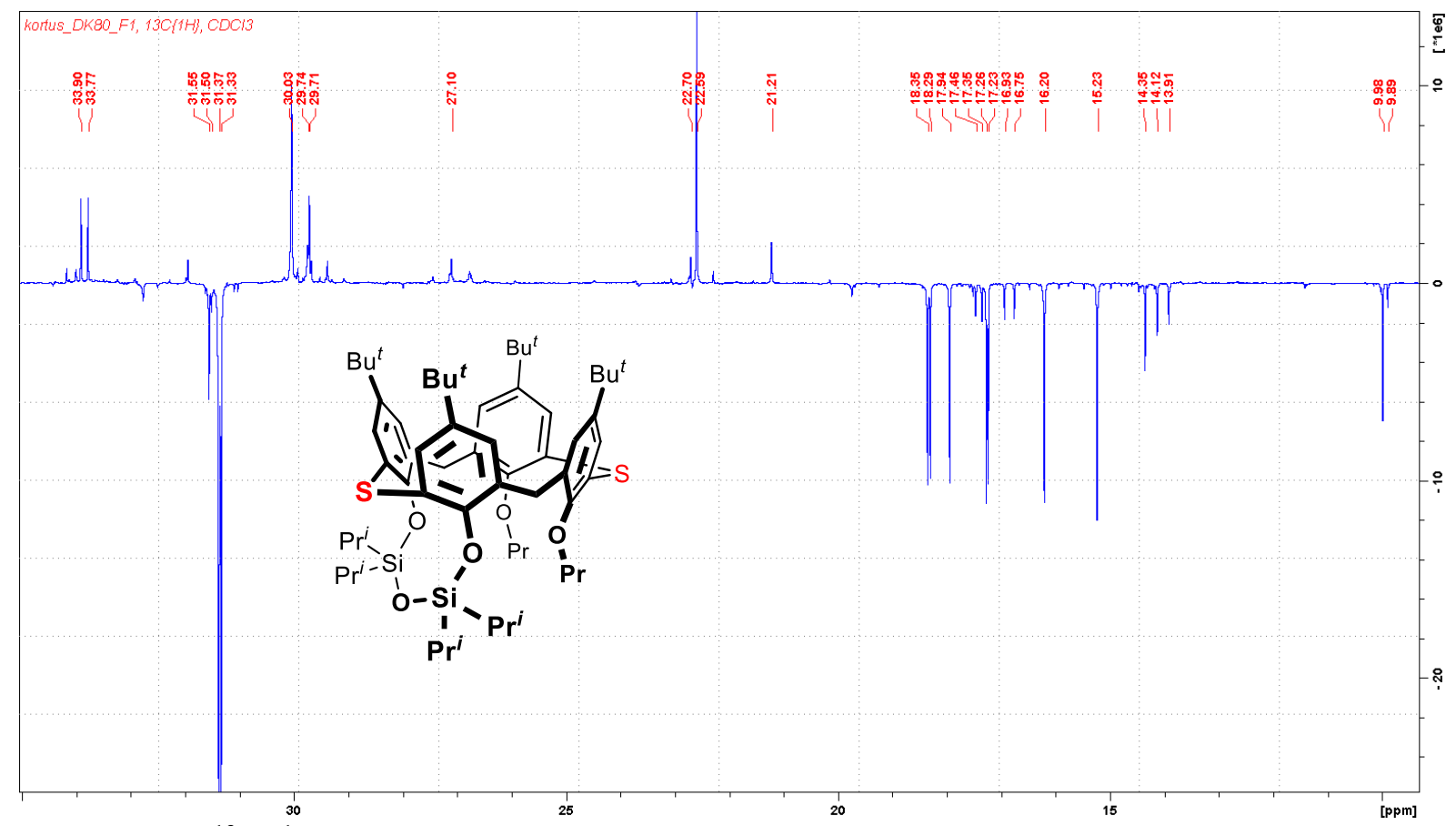

Figure S55. ${ }^{13} \mathrm{C}\left\{{ }^{1} \mathrm{H}\right\}$ NMR of compound 6, aliphatic region $\left(\mathrm{CDCl}_{3}, 151 \mathrm{MHz}\right)$. 


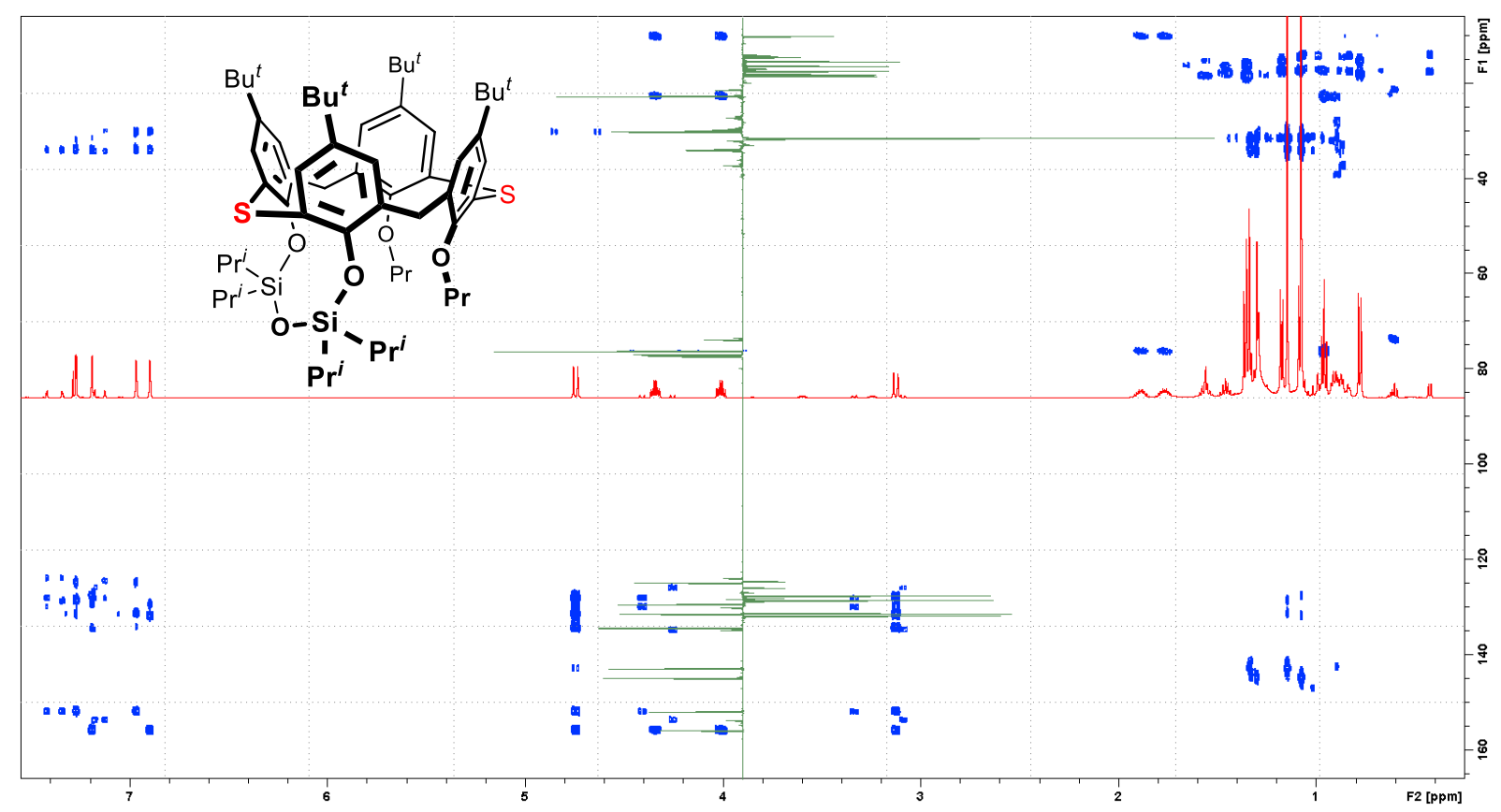

Figure S56. HMBC NMR of compound $6\left(\mathrm{CDCl}_{3}\right)$.

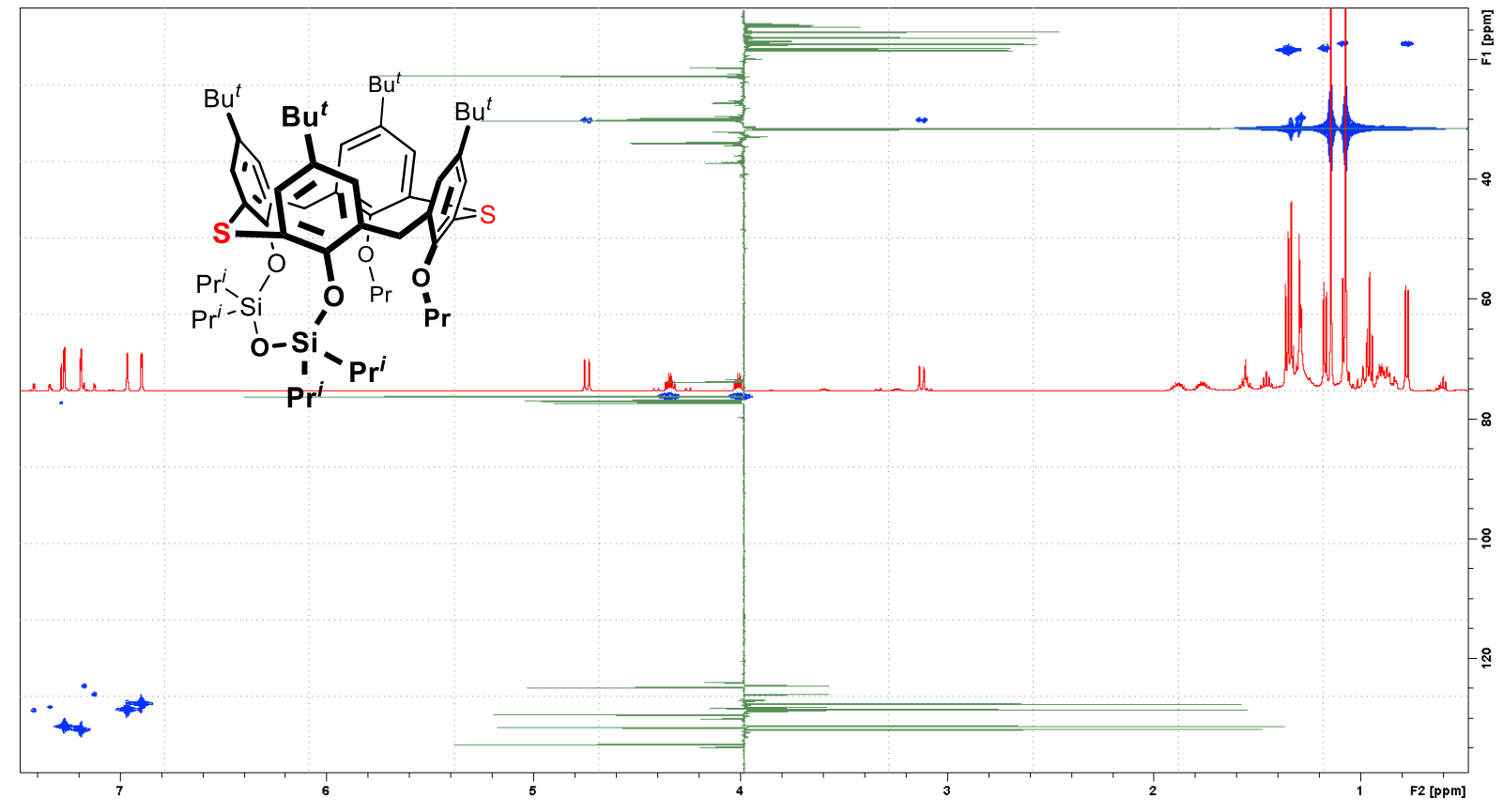

Figure S57. HMQC NMR of compound $6\left(\mathrm{CDCl}_{3}\right)$. 


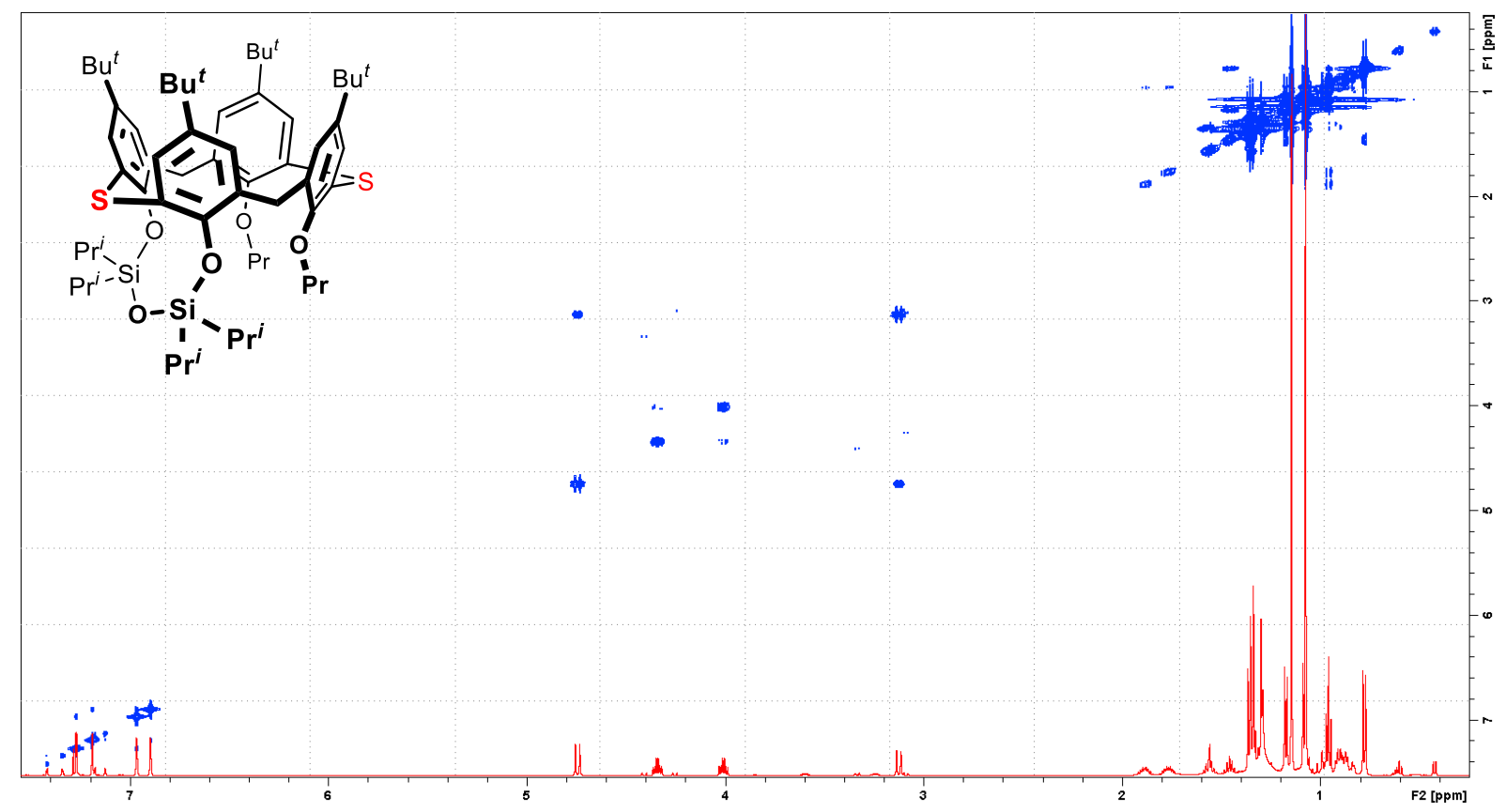

Figure S58. COSY NMR of compound $6\left(\mathrm{CDCl}_{3}\right)$.

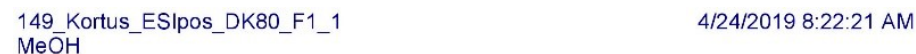

149_Kortus_ESIpos_DK80_F1_1 \#81-87 RT: 1.17-1.26 AV: 7 NL: 3.24E7

T: FTMS + c ESI Full ms [200.00-2000.00]

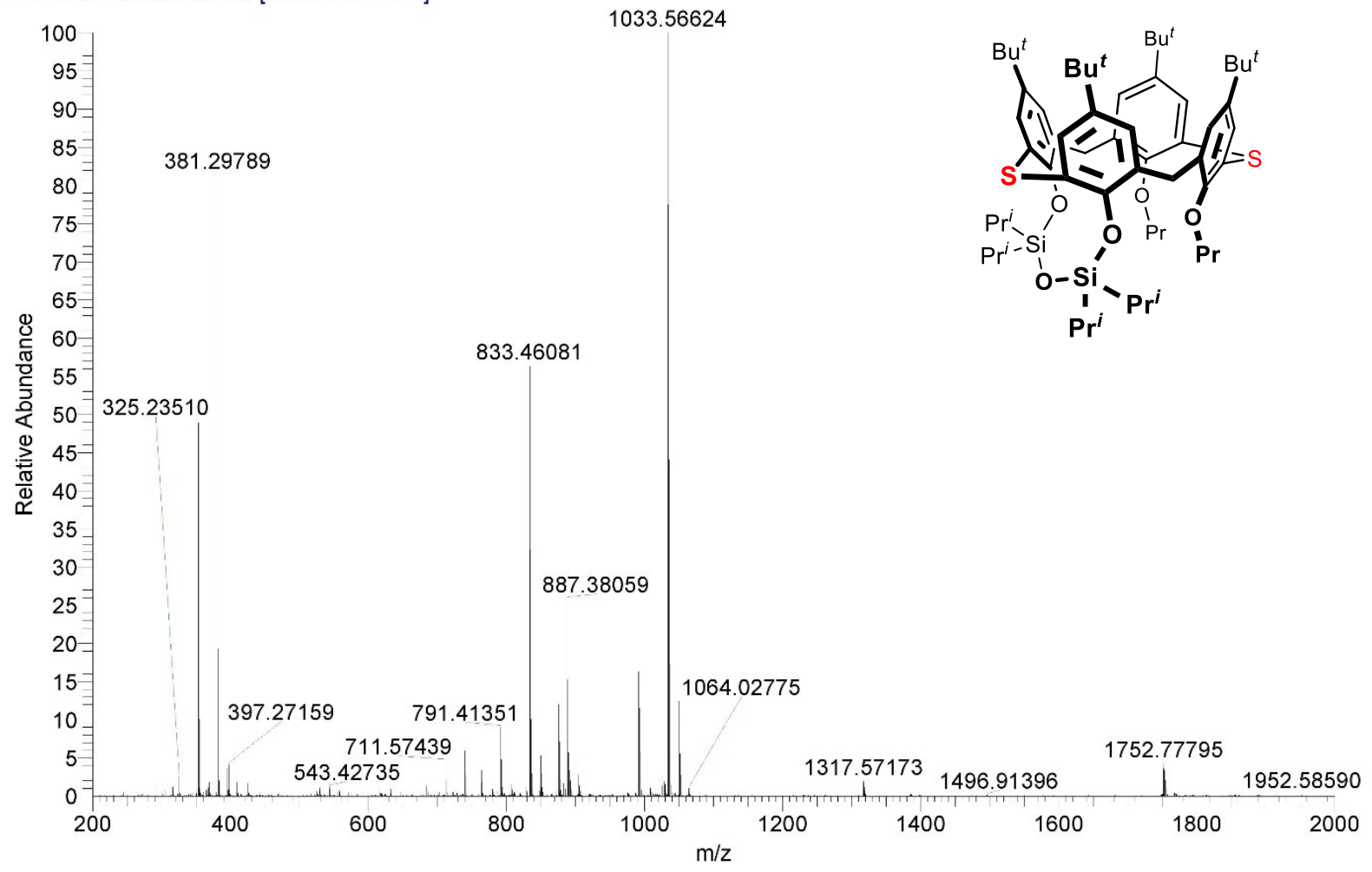

Figure S59. HRMS of compound $6\left(\mathrm{ESI}^{+}\right)$. 


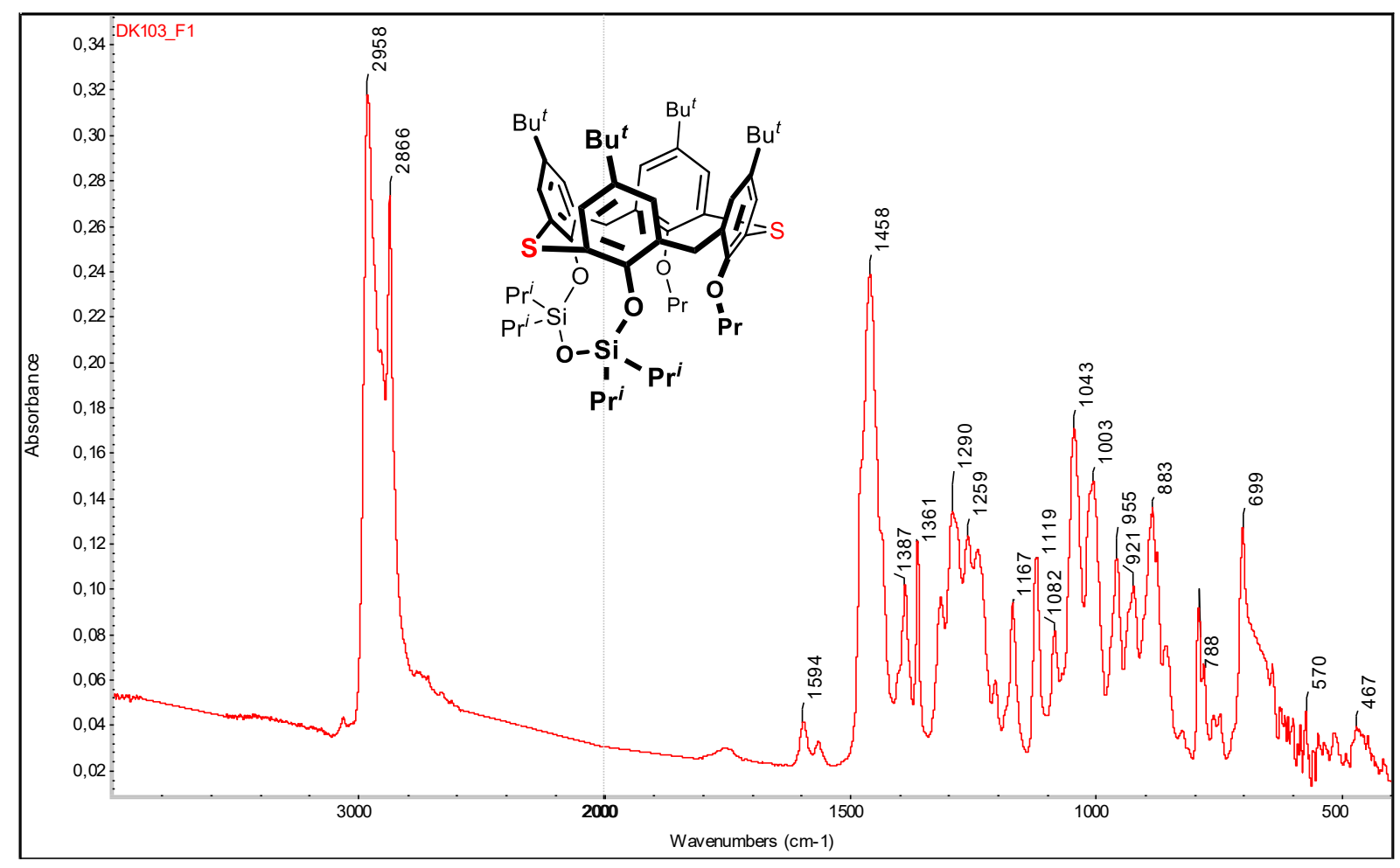

Figure S60. IR of compound 6 (ATR). 


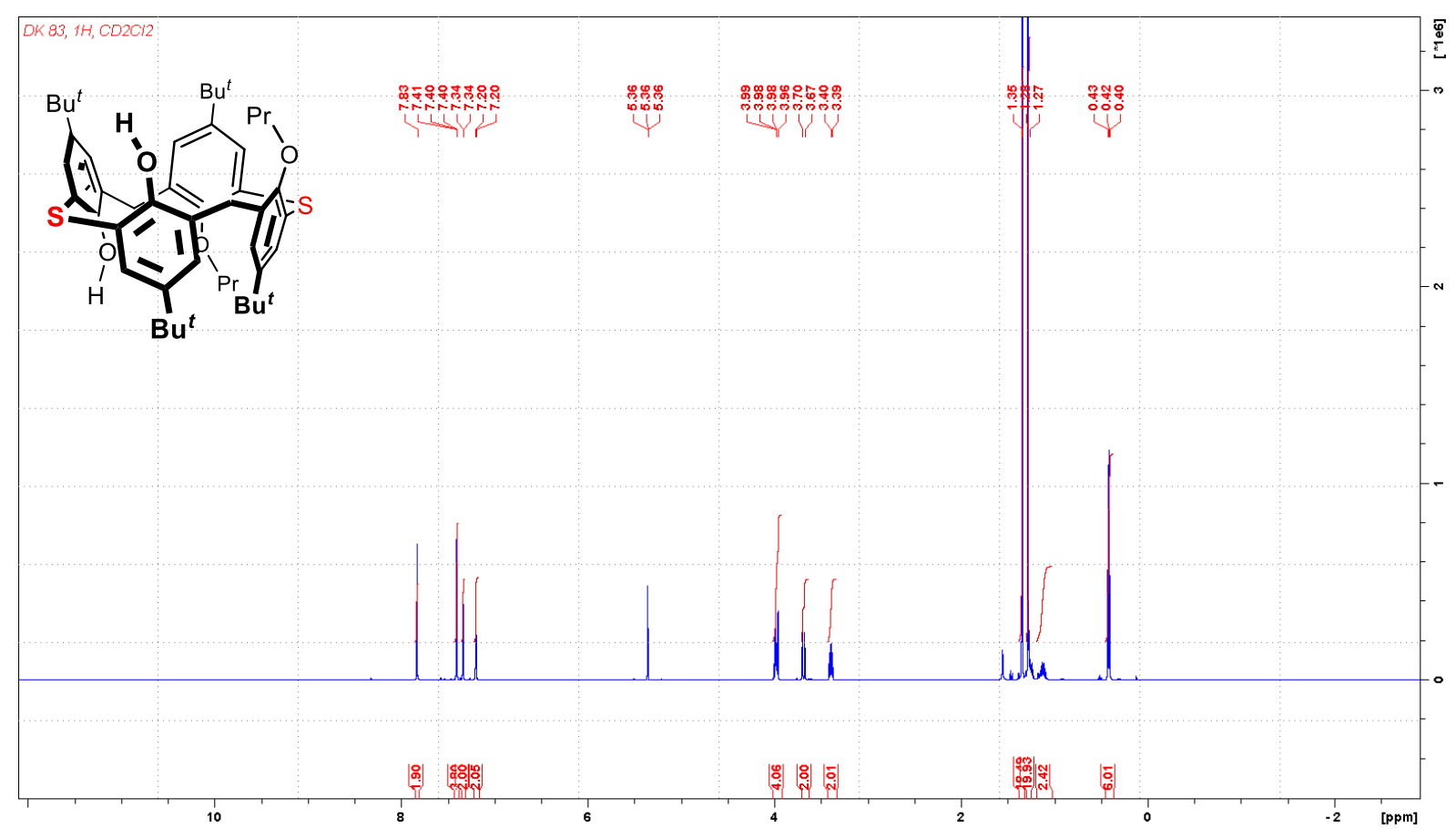

Figure S61. ${ }^{1} \mathrm{H}$ NMR of compound $7\left(\mathrm{CD}_{2} \mathrm{Cl}_{2}, 600 \mathrm{MHz}\right)$.

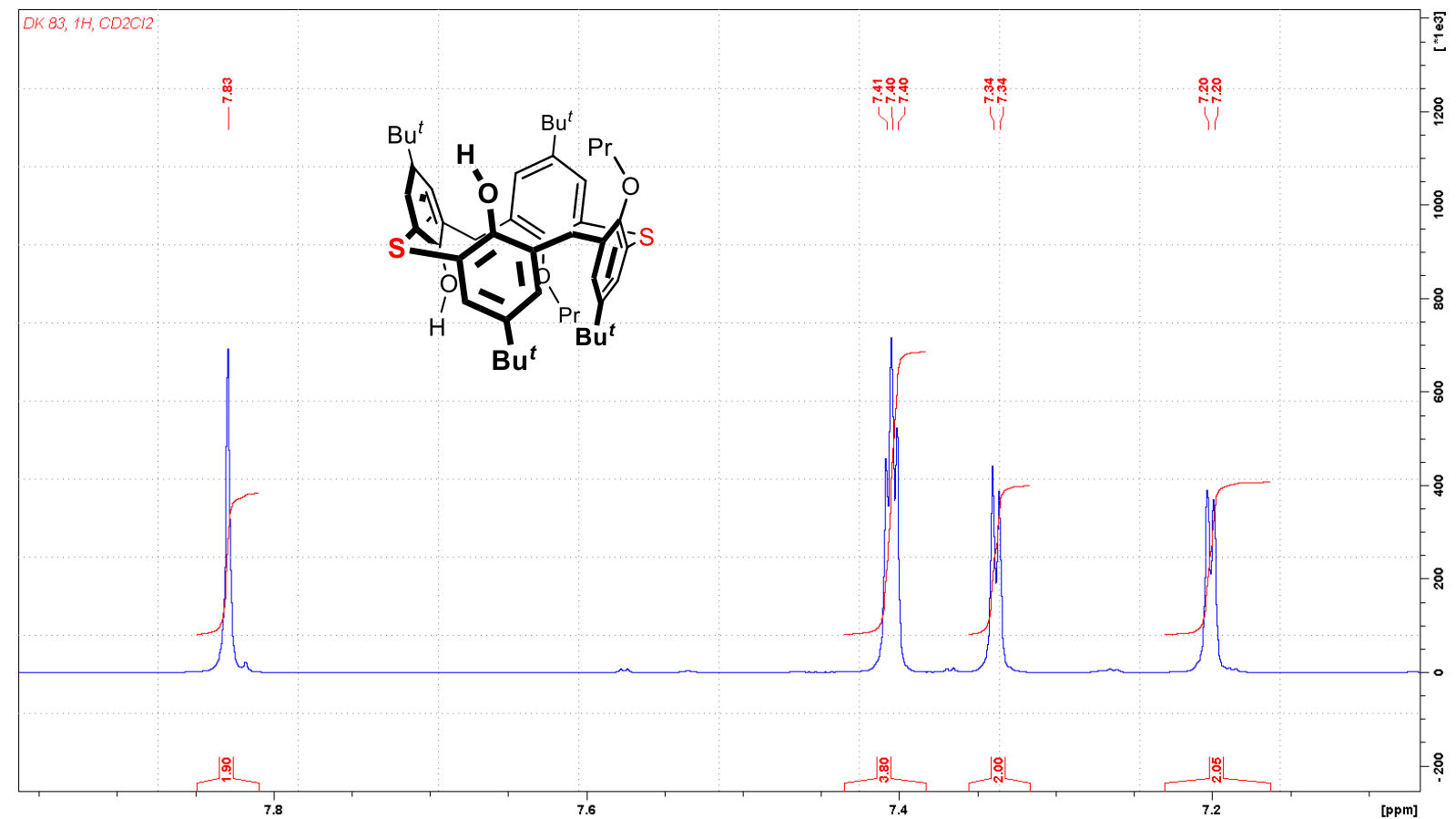

Figure S62. ${ }^{1} \mathrm{H}$ NMR of compound 7, aromatic region $\left(\mathrm{CD}_{2} \mathrm{Cl}_{2}, 600 \mathrm{MHz}\right)$. 


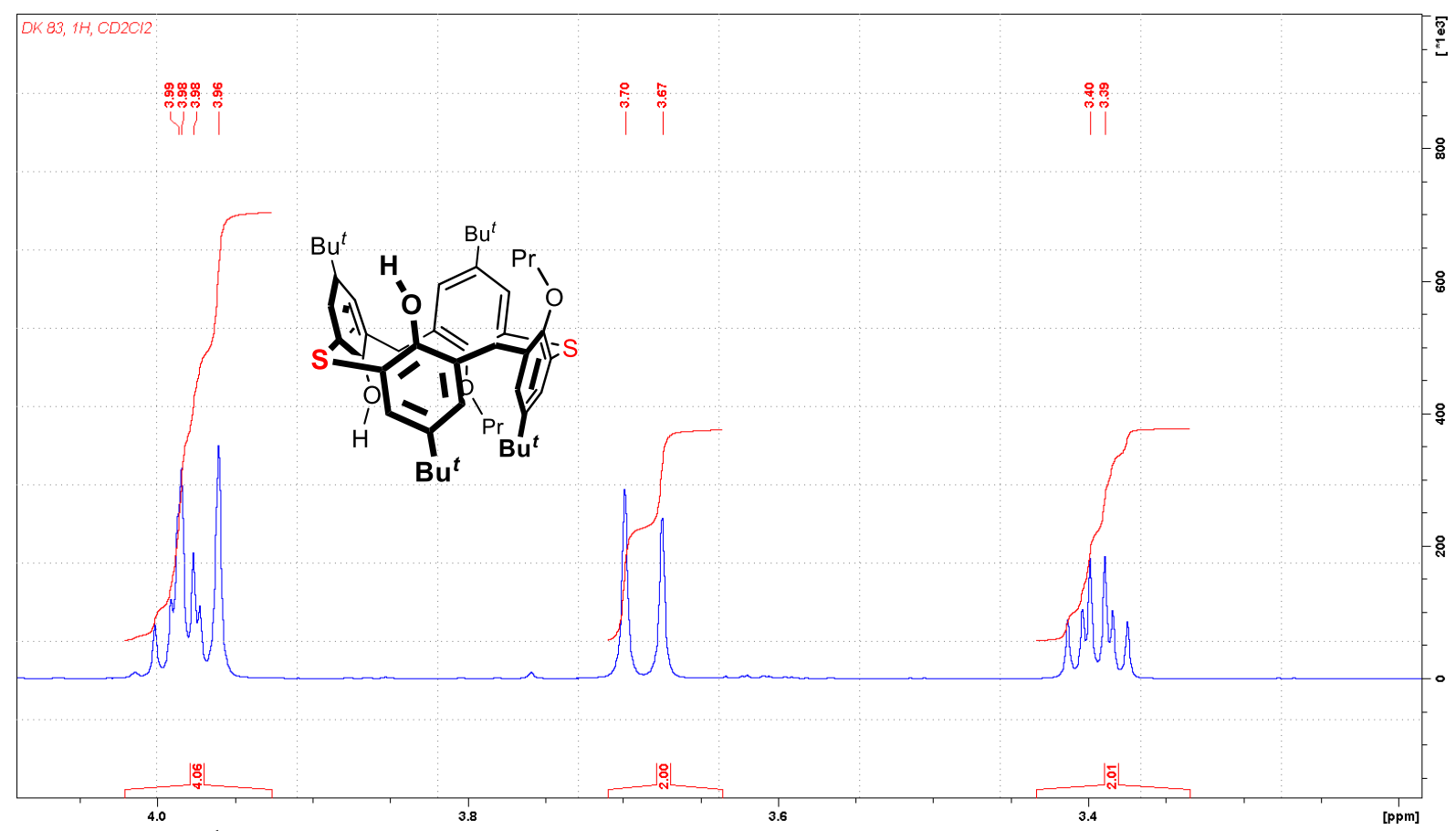

Figure S63. ${ }^{1} \mathrm{H} \mathrm{NMR}$ of compound 7, bridge region $\left(\mathrm{CD}_{2} \mathrm{Cl}_{2}, 600 \mathrm{MHz}\right)$.

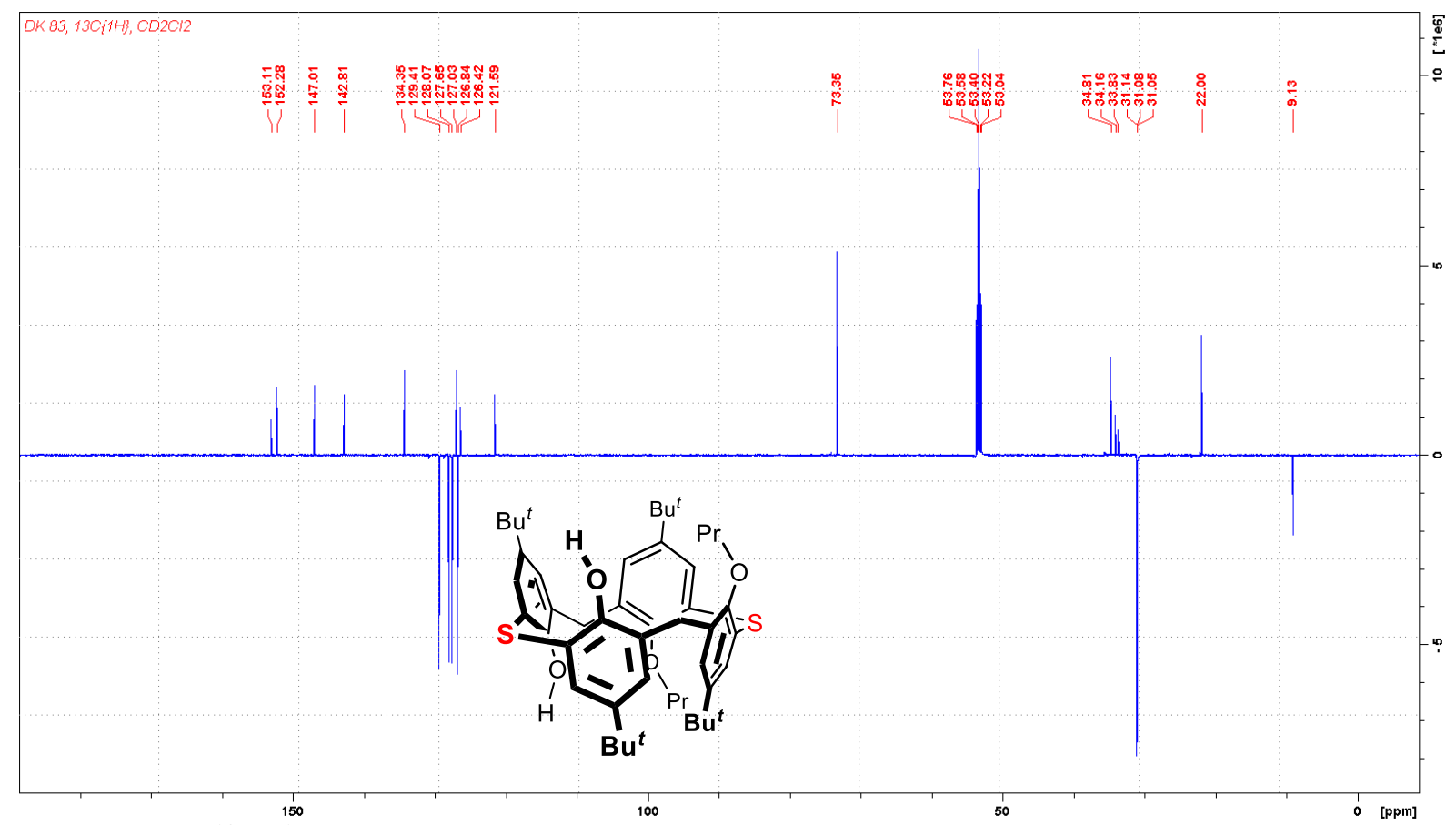

Figure S64. ${ }^{13} \mathrm{C}\left\{{ }^{1} \mathrm{H}\right\}$ NMR of compound $7\left(\mathrm{CD}_{2} \mathrm{Cl}_{2}, 151 \mathrm{MHz}\right)$. 


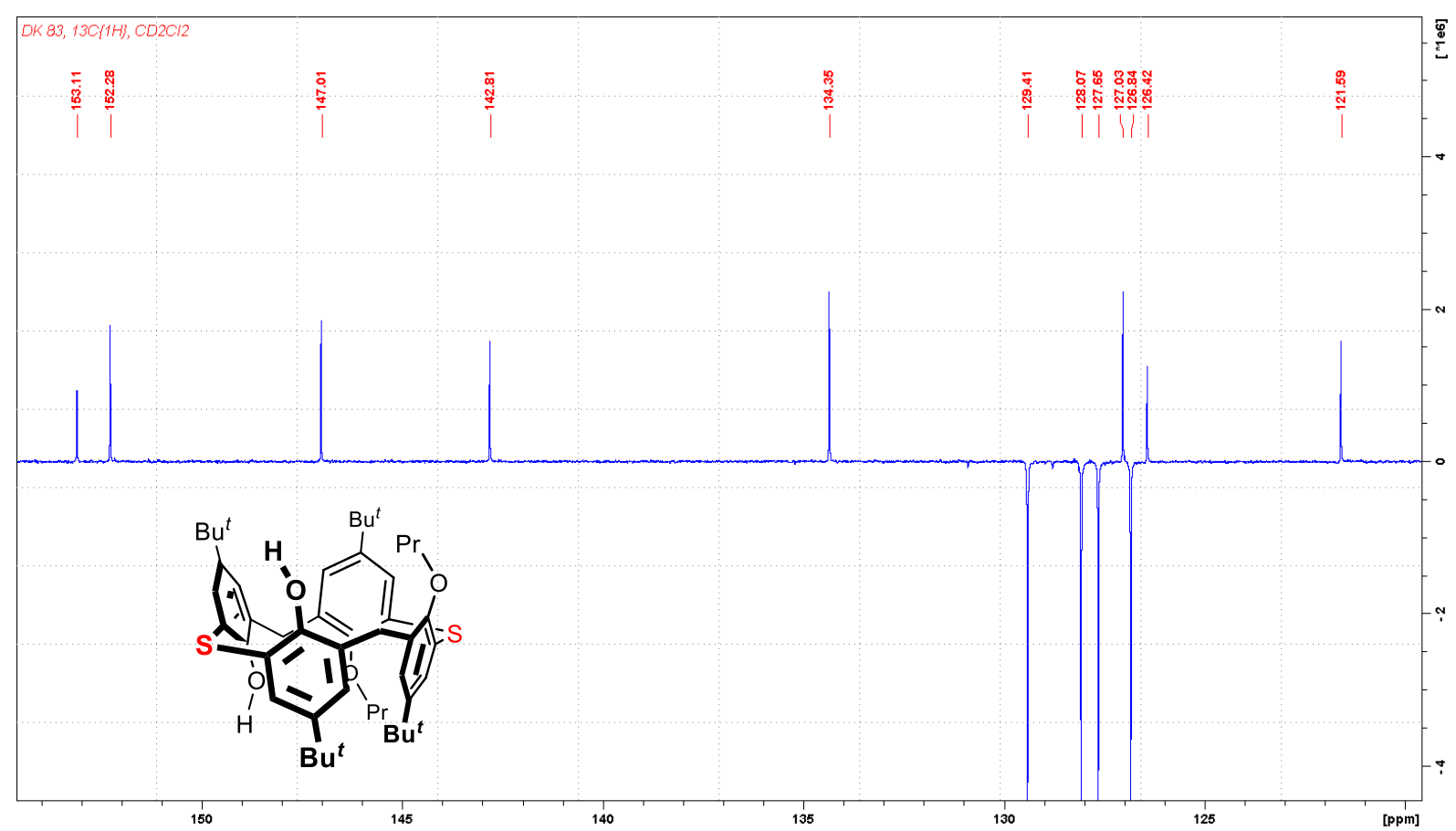

Figure S65. ${ }^{13} \mathrm{C}\left\{{ }^{1} \mathrm{H}\right\}$ NMR of compound 7, aromatic region $\left(\mathrm{CD}_{2} \mathrm{Cl}_{2}, 151 \mathrm{MHz}\right)$.

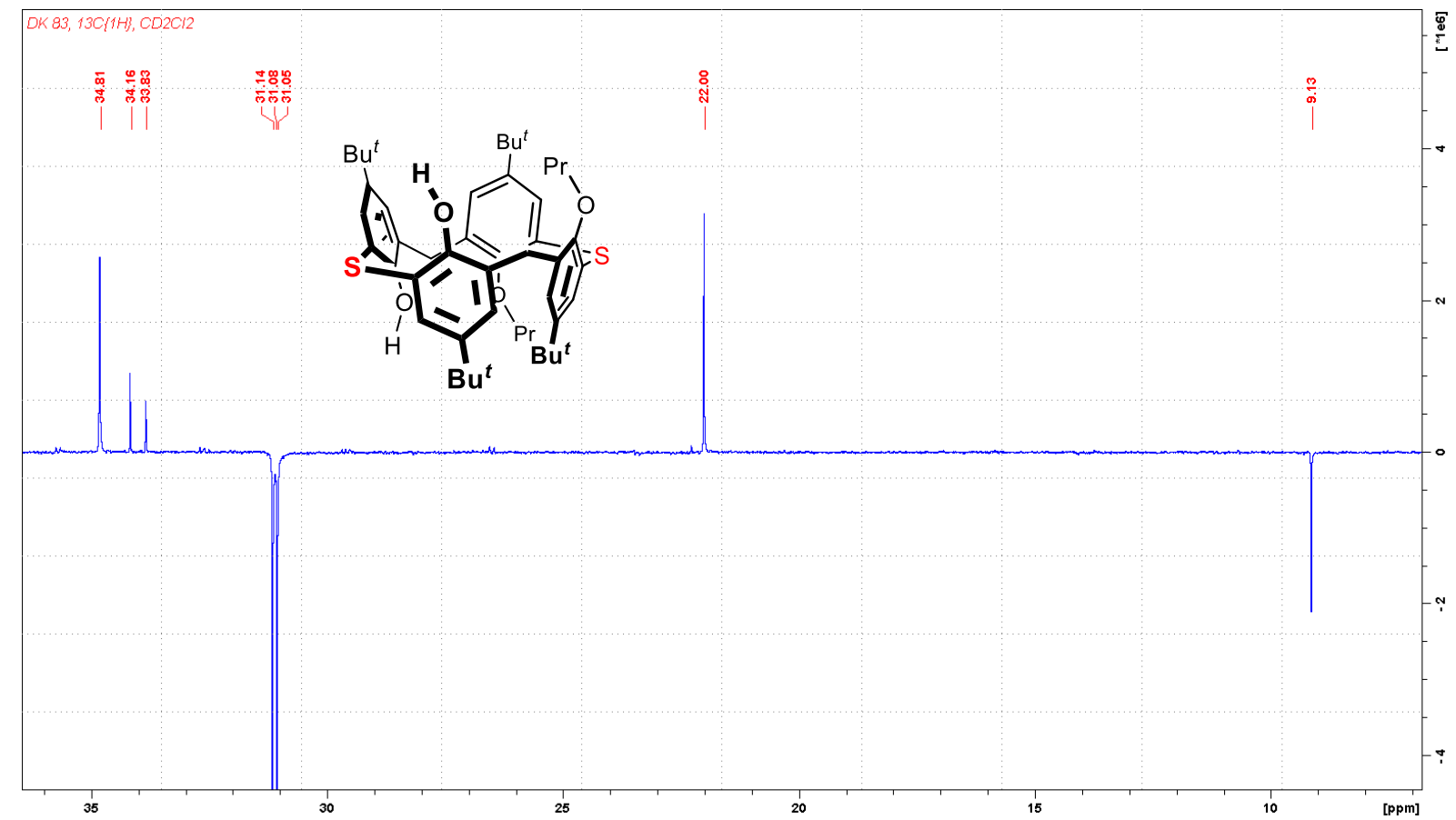

Figure S66. ${ }^{13} \mathrm{C}\left\{{ }^{1} \mathrm{H}\right\}$ NMR of compound 7, aliphatic region $\left(\mathrm{CD}_{2} \mathrm{Cl}_{2}, 151 \mathrm{MHz}\right)$. 


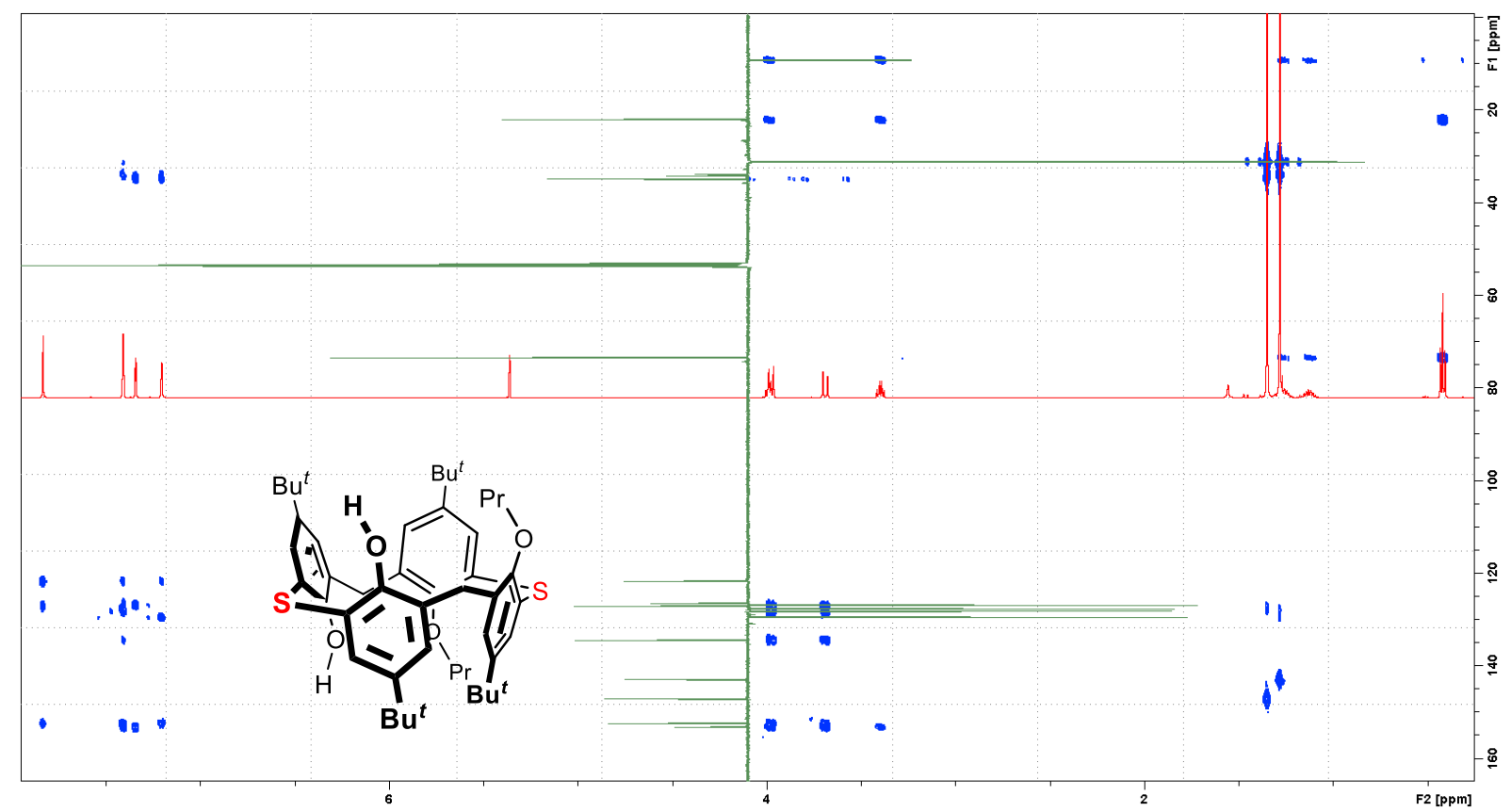

Figure S67. HMBC NMR of compound $7\left(\mathrm{CDCl}_{3}\right)$.

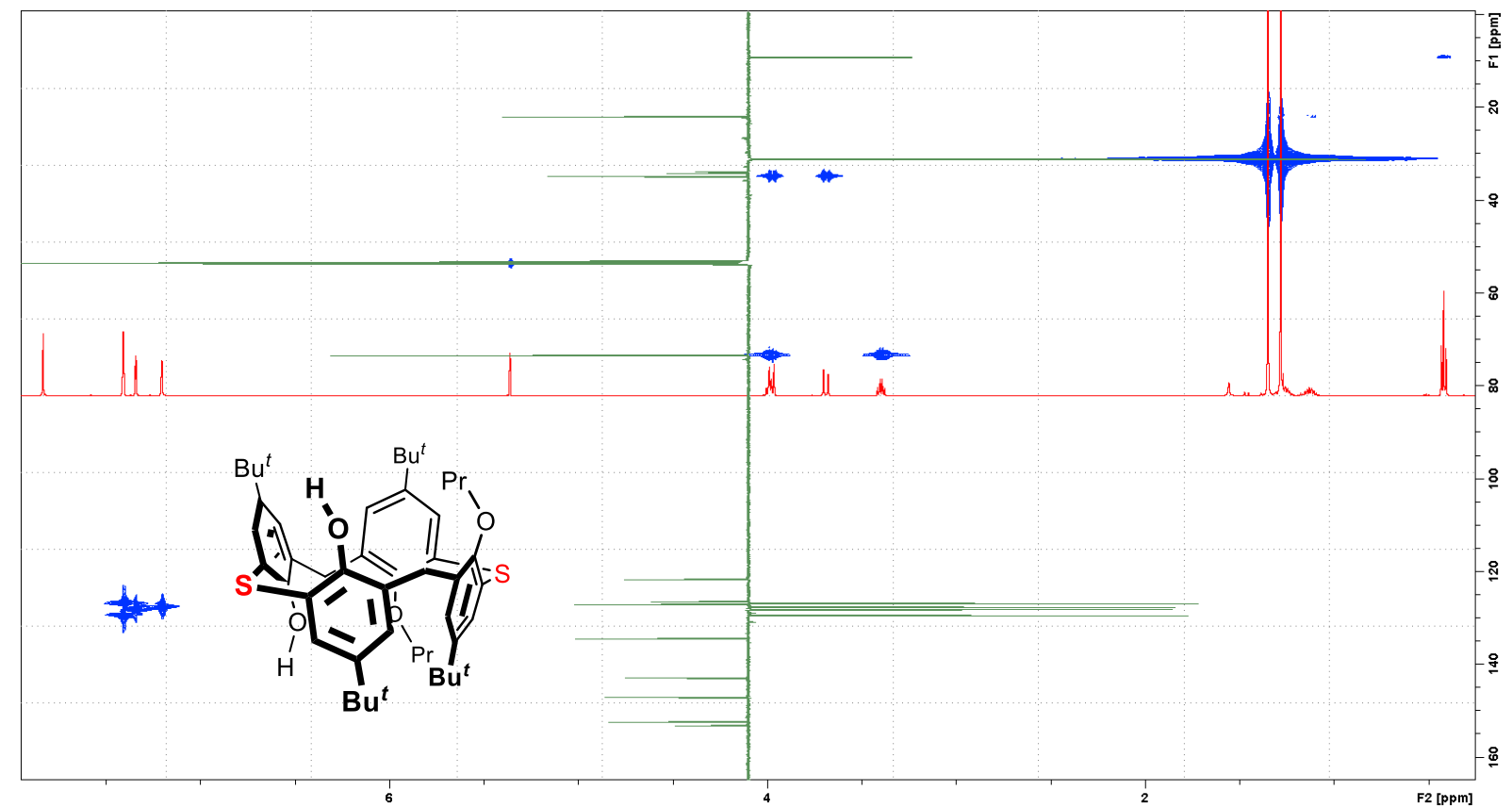

Figure S68. HMQC NMR of compound $7\left(\mathrm{CDCl}_{3}\right)$. 


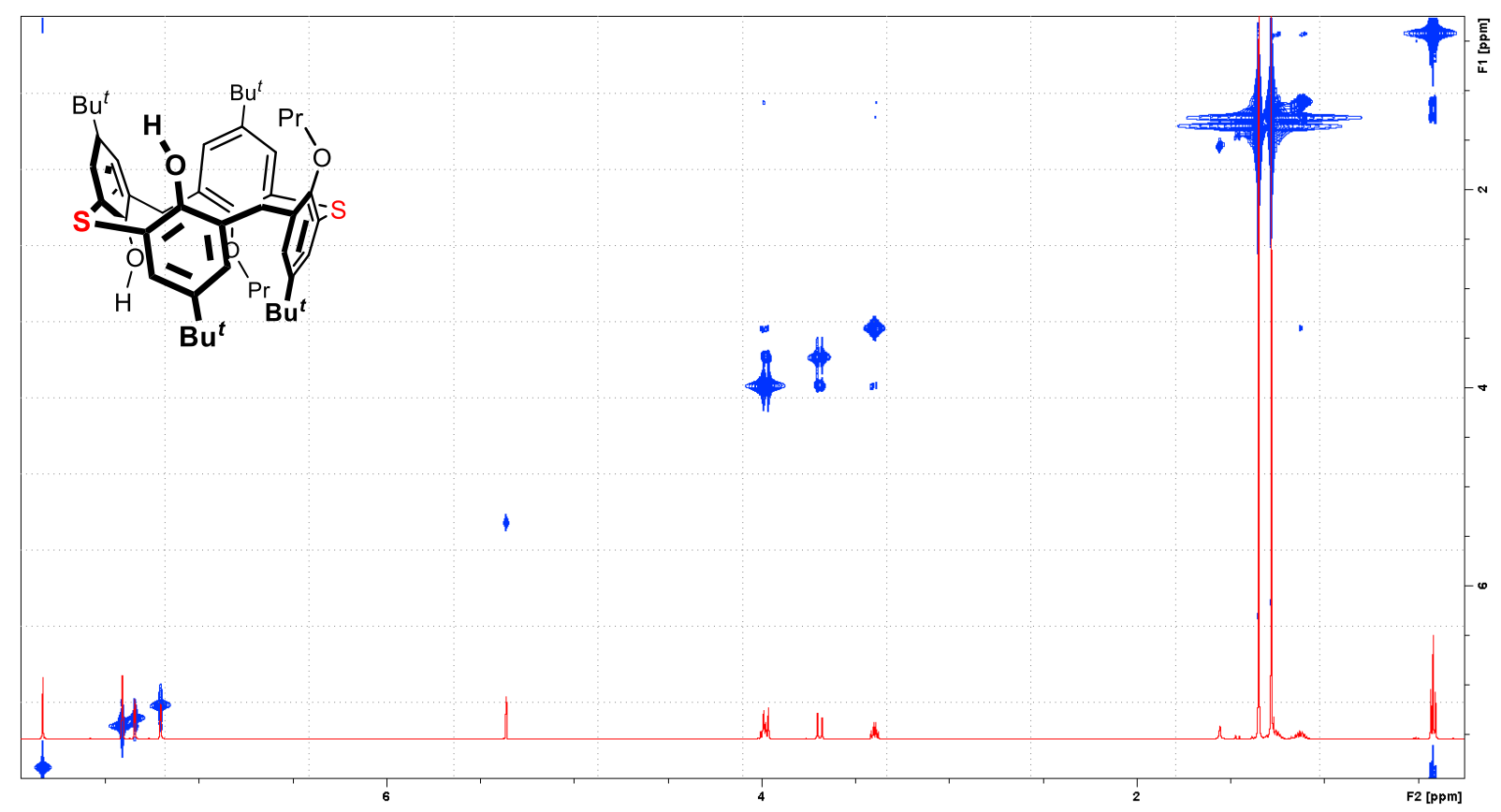

Figure S69. COSY NMR of compound $7\left(\mathrm{CDCl}_{3}\right)$.

155_Kortus_ESIpos_DK83_1 $\mathrm{Me} \overline{\mathrm{H}}$

4/26/2019 8:14:18 AM

155_Kortus_ESIpos_DK83_1 \#52-57 RT: 0.75-0.82 AV: 6 NL: 4.92E7

T: FTMS + c ESI Full ms [200.00-2000.00]

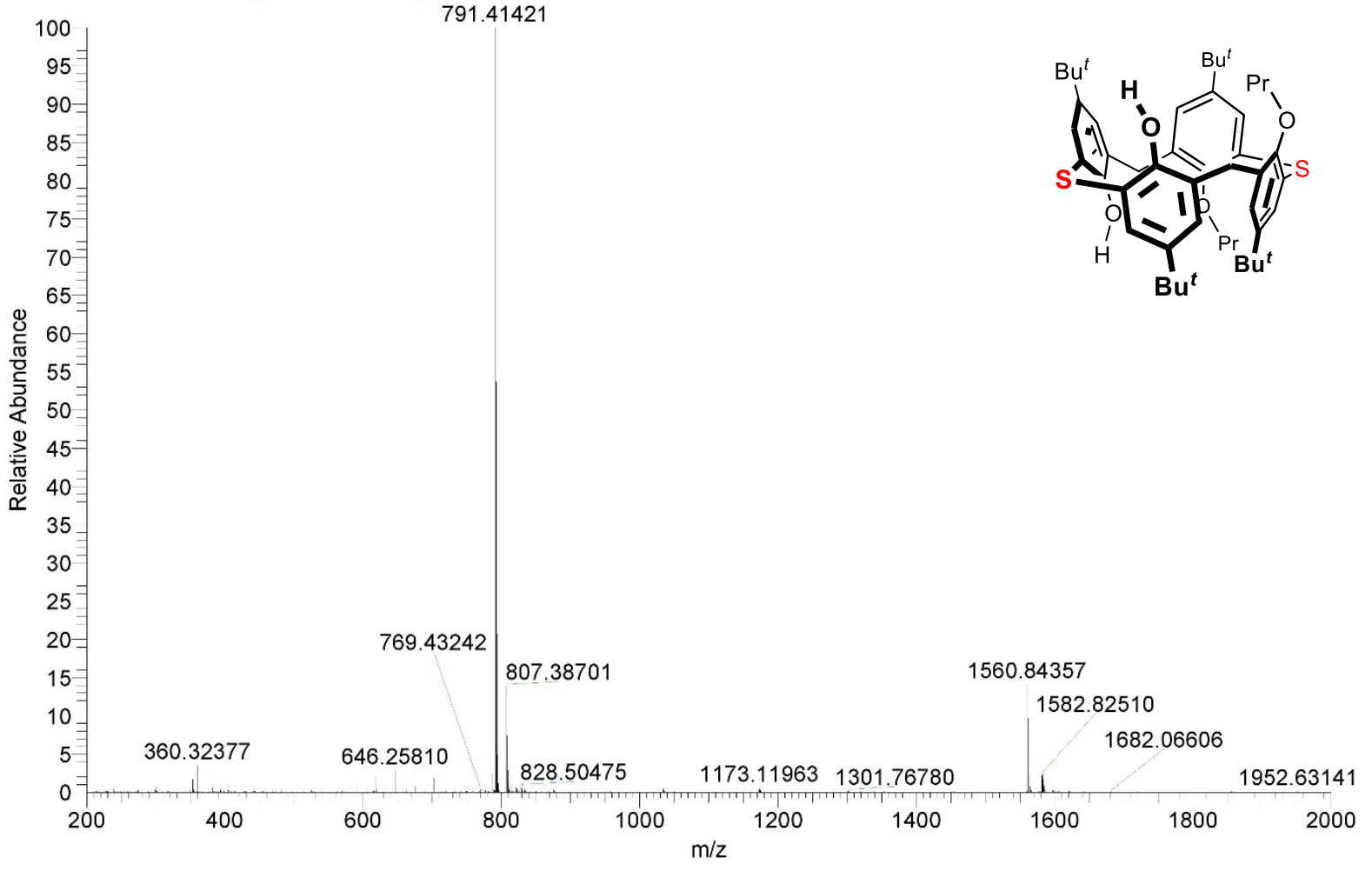

Figure S70. HRMS of compound $7\left(\mathrm{ESI}^{+}\right)$. 


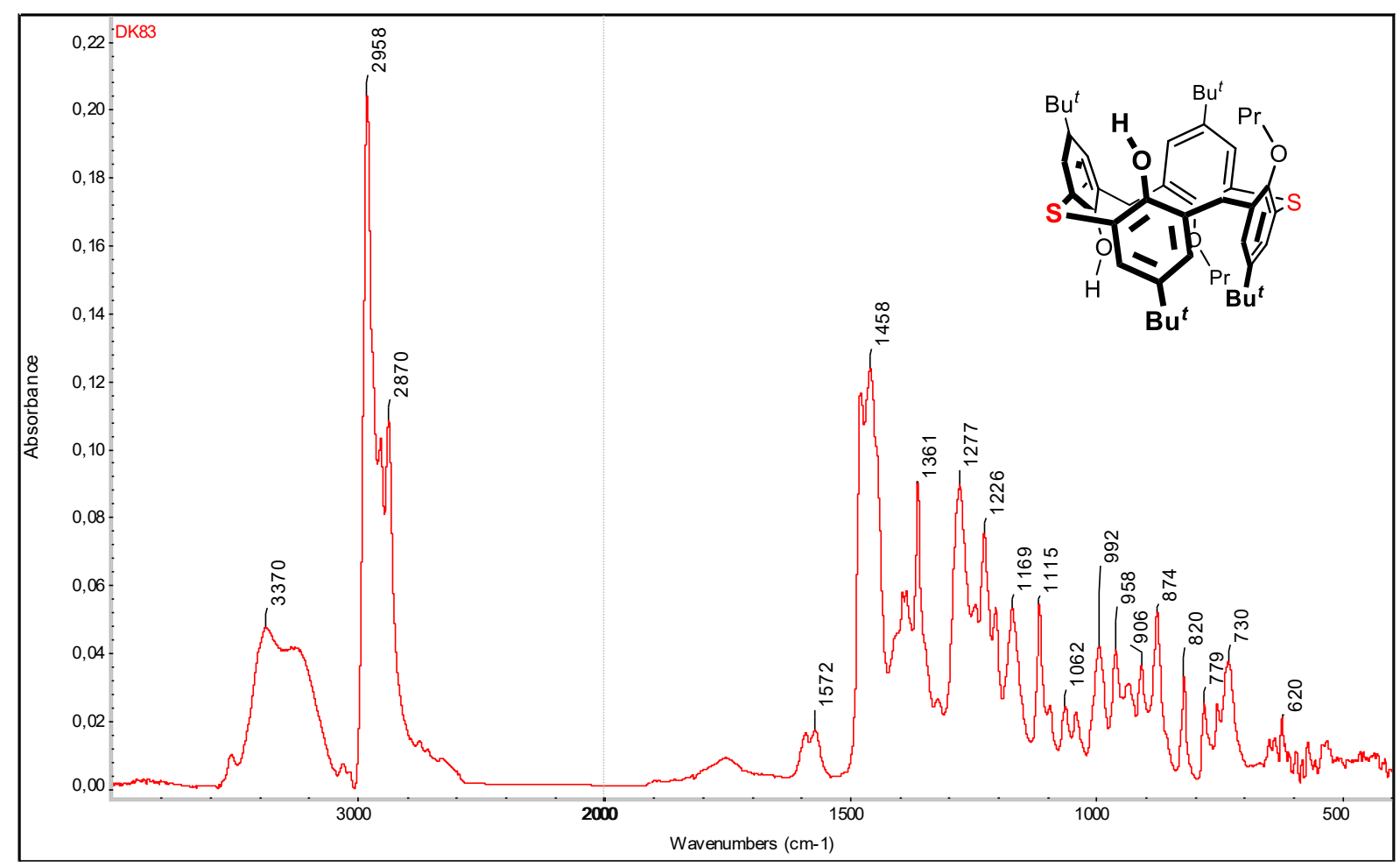

Figure S71. IR of compound 7 (ATR). 


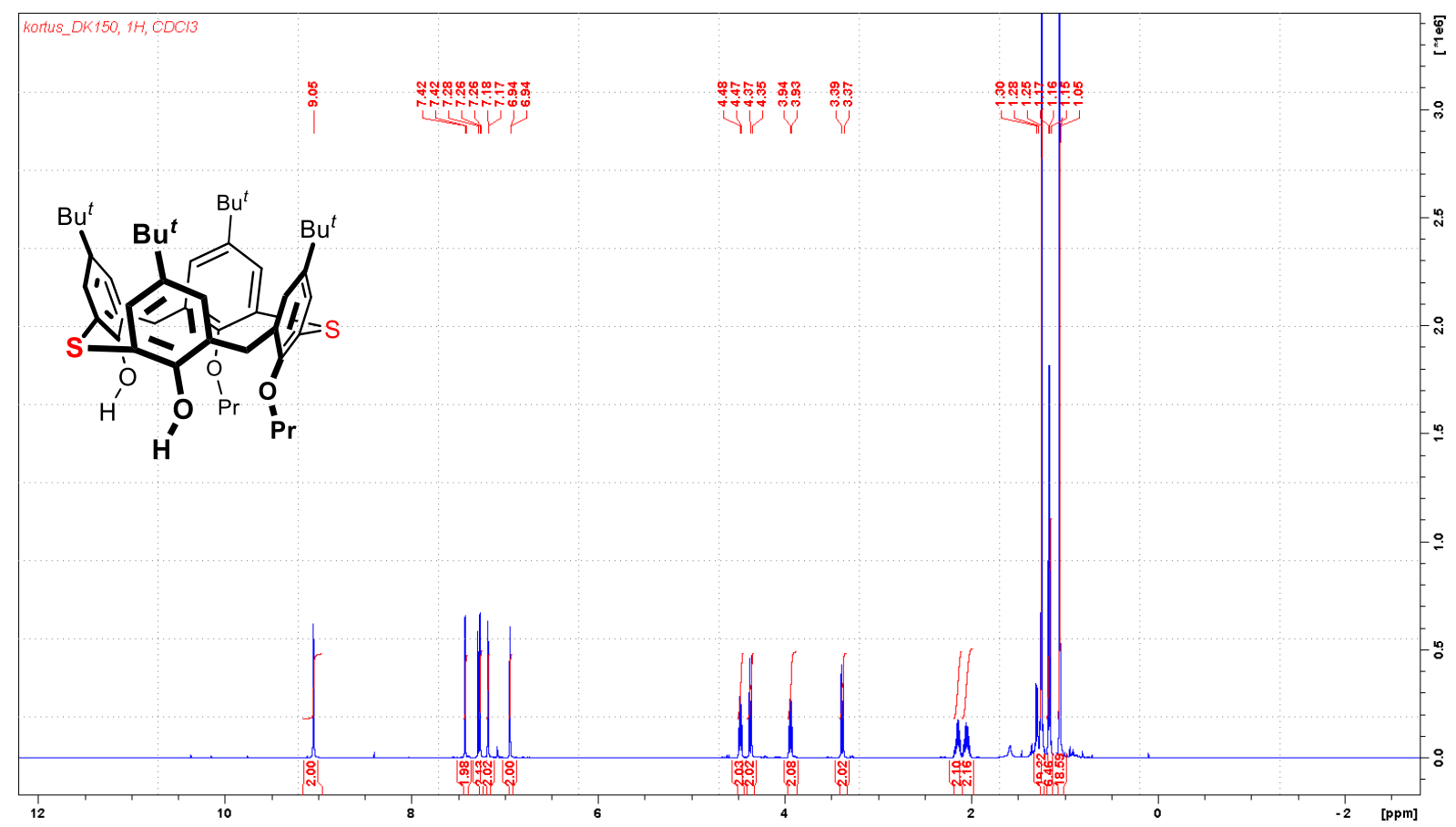

Figure S72. ${ }^{1} \mathrm{H} \mathrm{NMR}$ of compound $8\left(\mathrm{CDCl}_{3}, 600 \mathrm{MHz}\right)$.

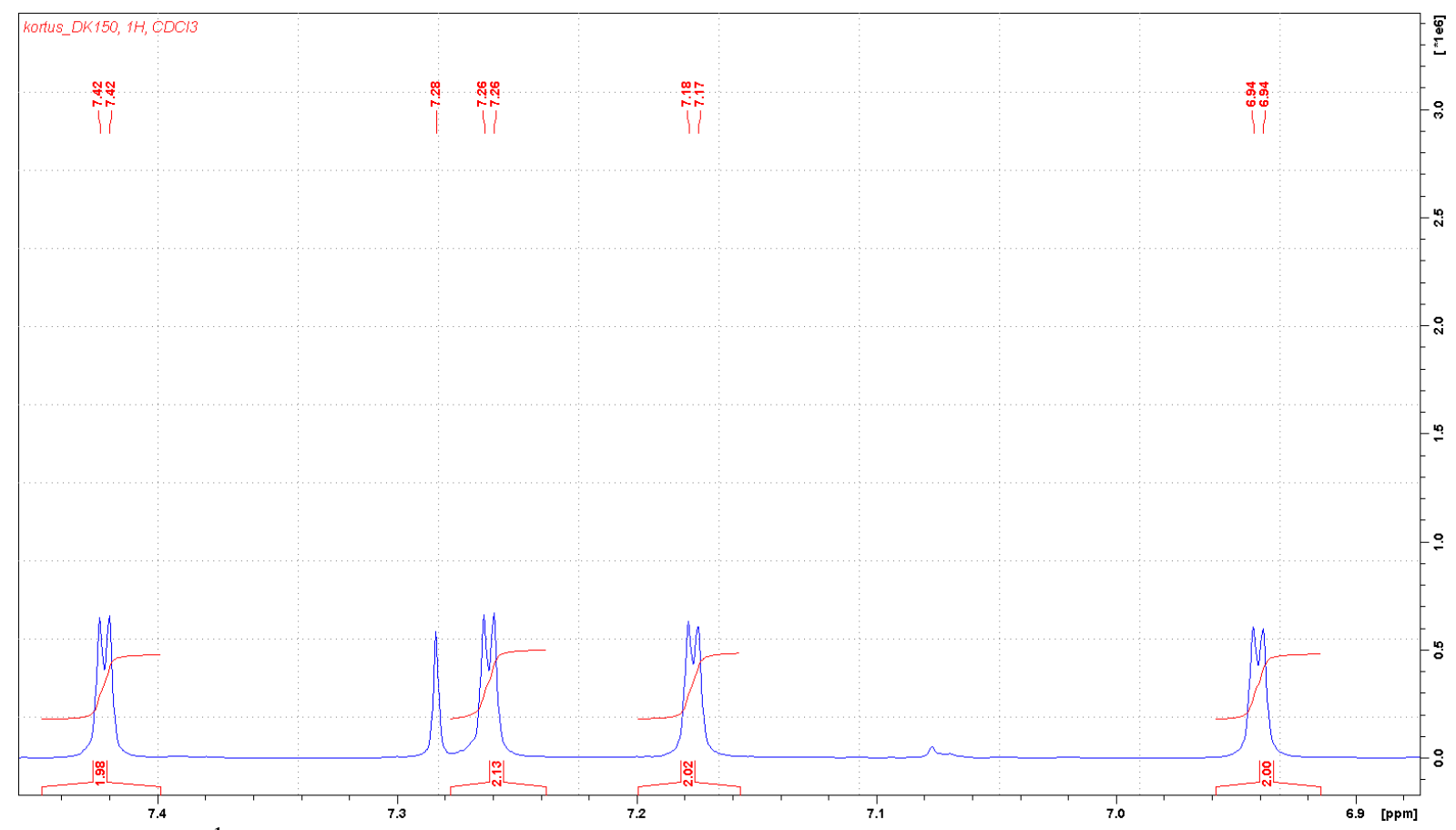

Figure S73. ${ }^{1} \mathrm{H}$ NMR of compound 8 , aromatic region $\left(\mathrm{CDCl}_{3}, 600 \mathrm{MHz}\right)$. 


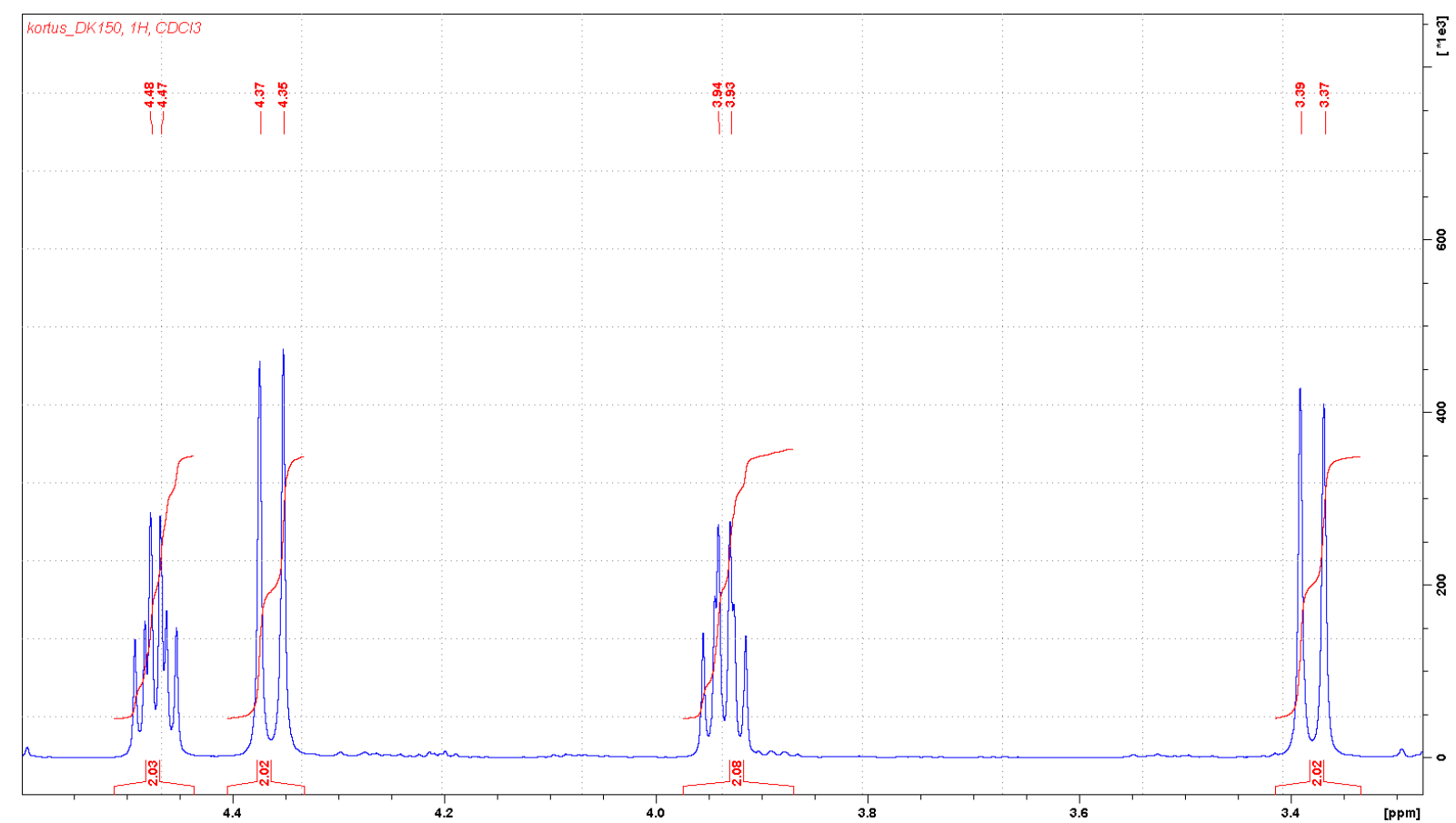

Figure S74. ${ }^{1} \mathrm{H}$ NMR of compound $\mathbf{8}$, bridge region $\left(\mathrm{CDCl}_{3}, 600 \mathrm{MHz}\right)$.

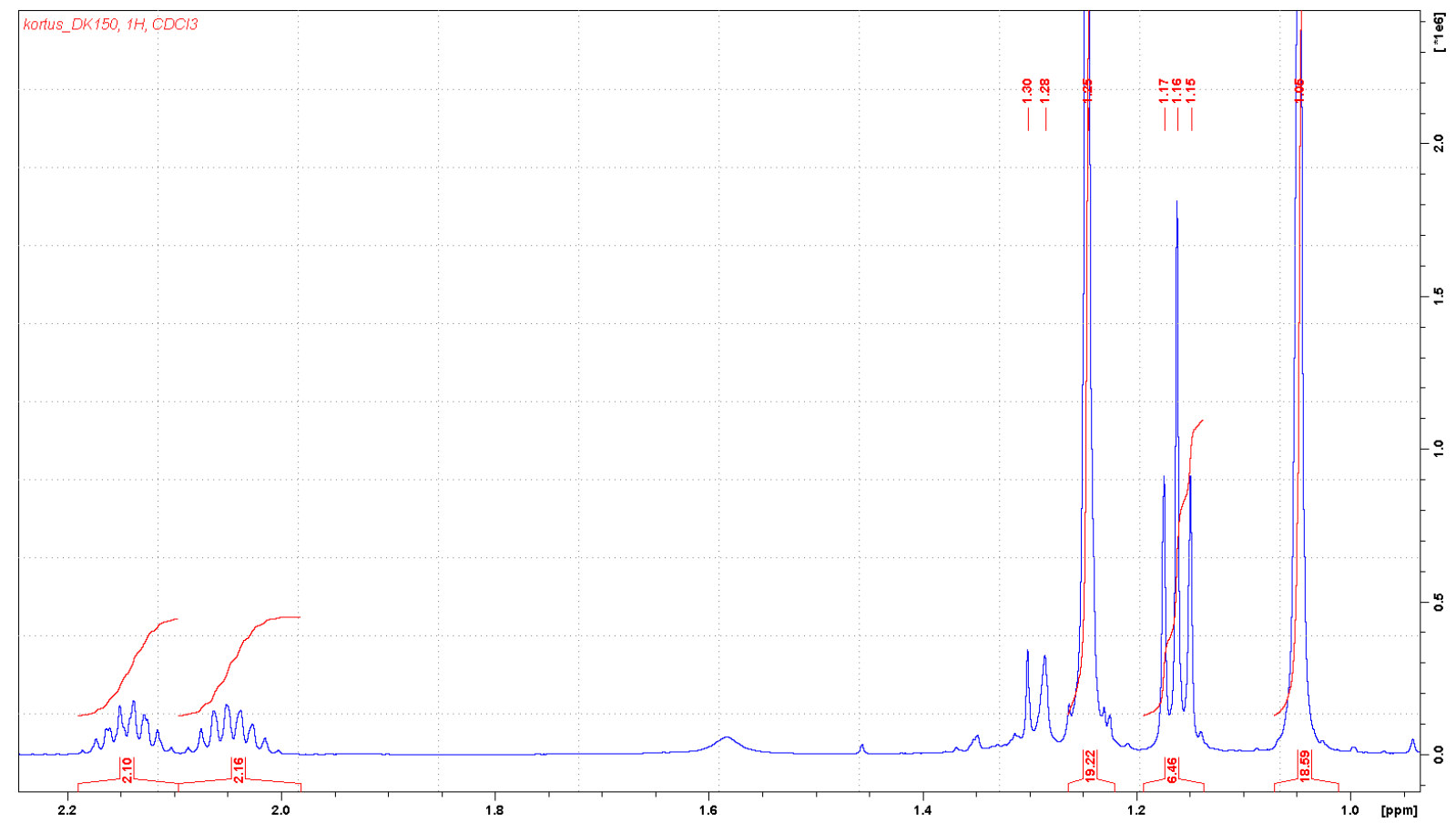

Figure S75. ${ }^{1} \mathrm{H} \mathrm{NMR}$ of compound 8, aliphatic region $\left(\mathrm{CDCl}_{3}, 600 \mathrm{MHz}\right)$. 


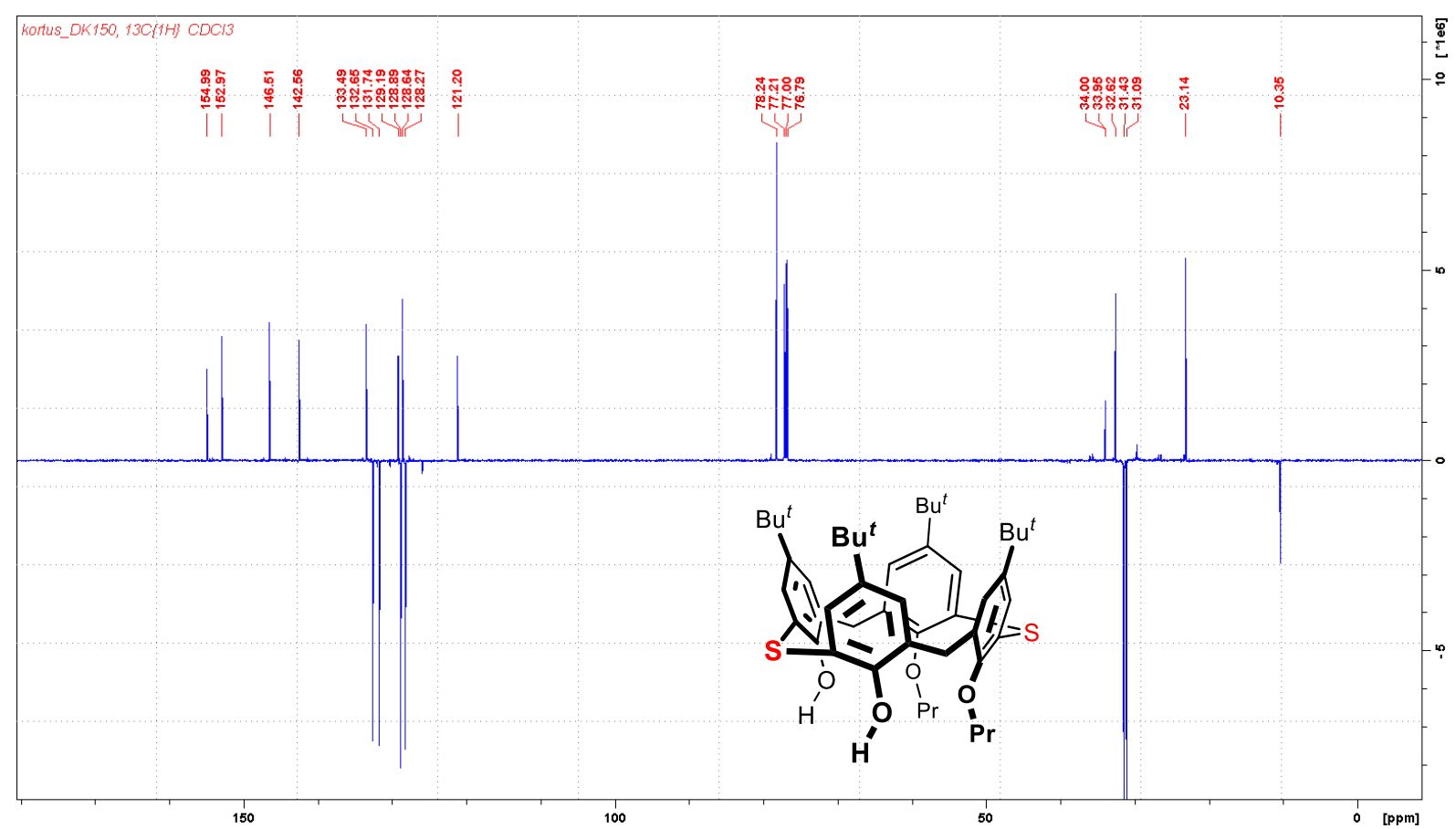

Figure S76. ${ }^{13} \mathrm{C}\left\{{ }^{1} \mathrm{H}\right\}$ NMR of compound $\mathbf{8}\left(\mathrm{CDCl}_{3}, 151 \mathrm{MHz}\right)$.

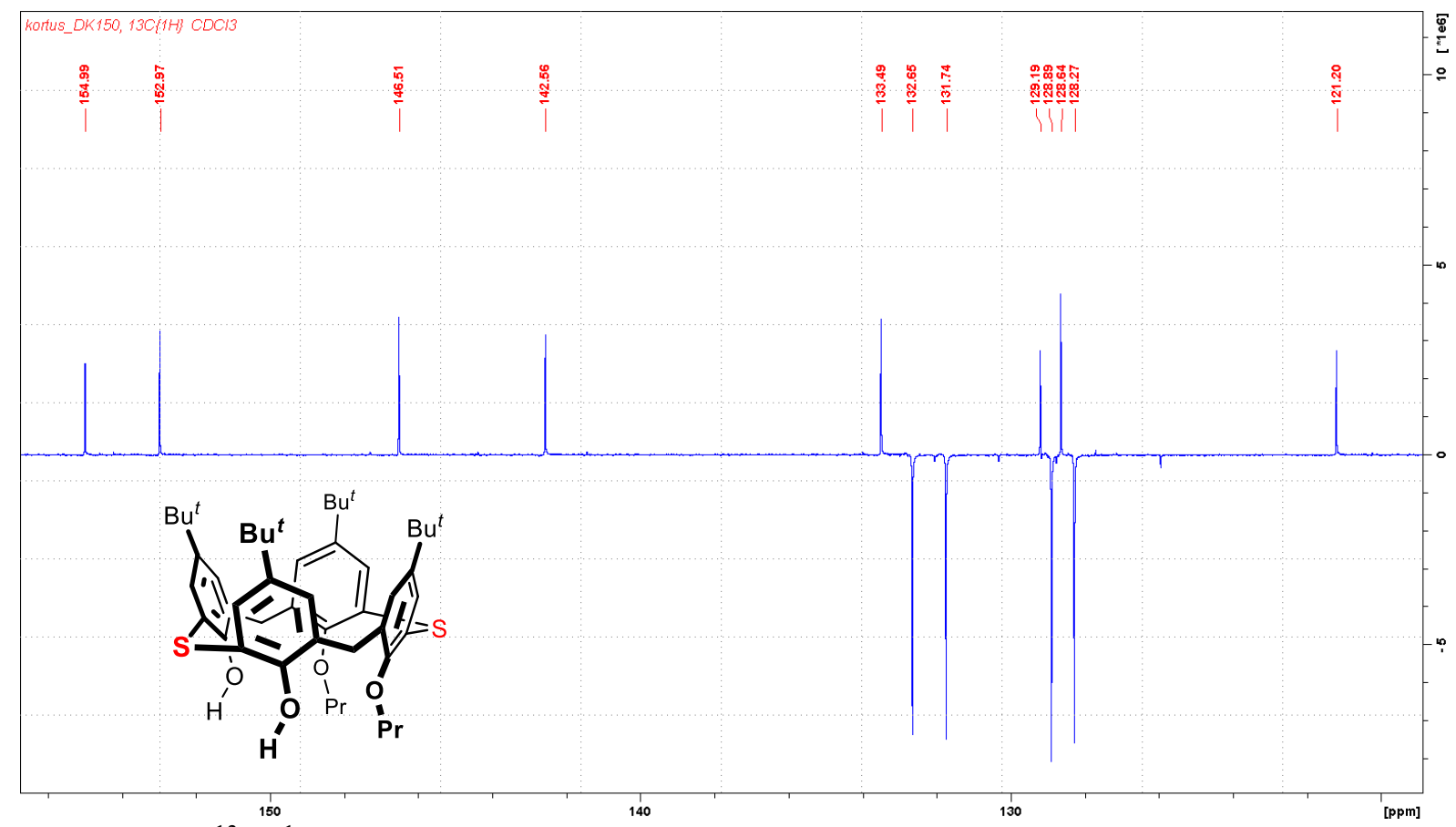

Figure S76. ${ }^{13} \mathrm{C}\left\{{ }^{1} \mathrm{H}\right\}$ NMR of compound 8 , aromatic region $\left(\mathrm{CDCl}_{3}, 151 \mathrm{MHz}\right)$. 


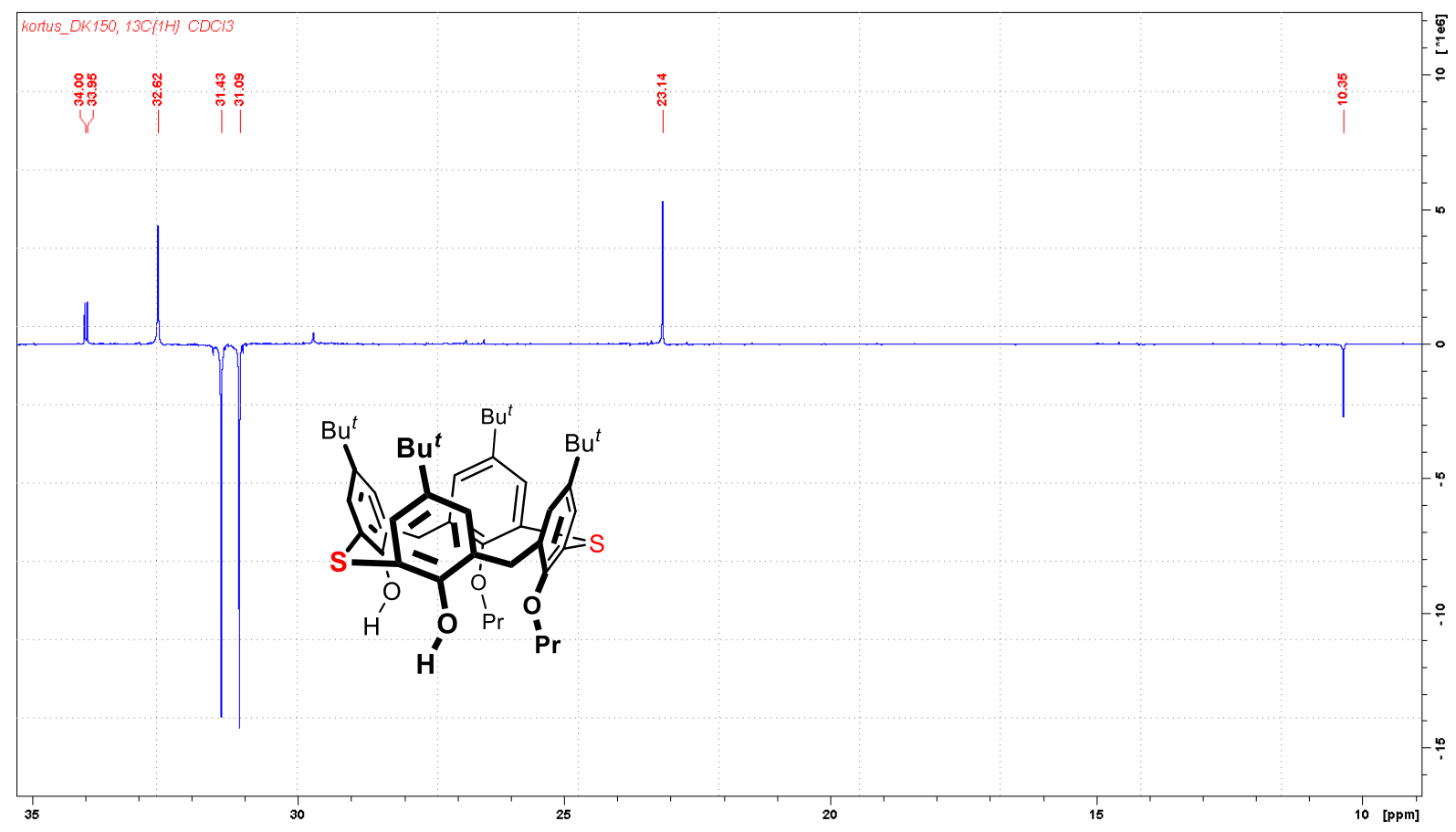

Figure S77. ${ }^{13} \mathrm{C}\left\{{ }^{1} \mathrm{H}\right\}$ NMR of compound 8, aliphatic region $\left(\mathrm{CDCl}_{3}, 151 \mathrm{MHz}\right)$.

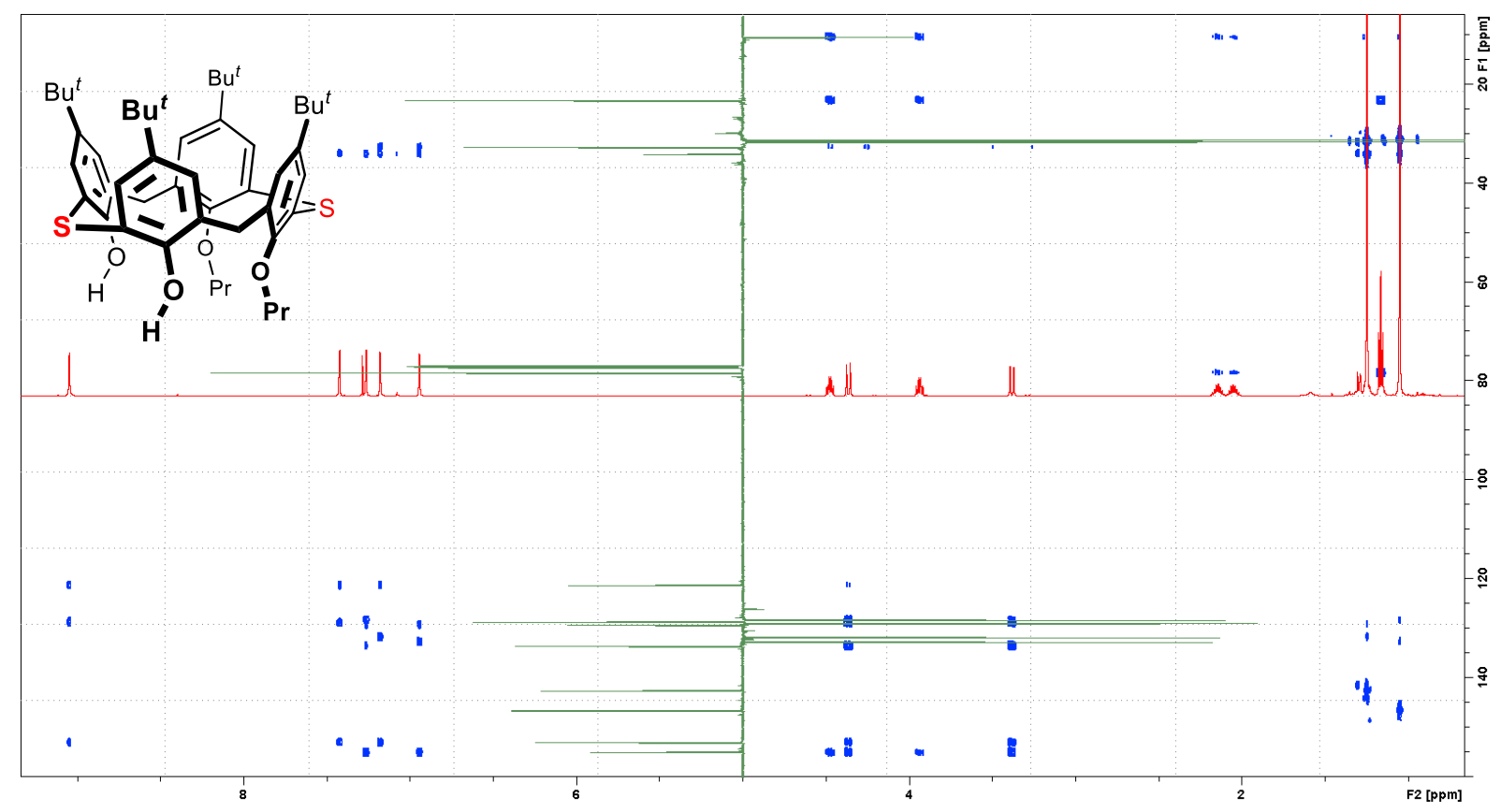

Figure S78. HMBC NMR of compound $\mathbf{8}\left(\mathrm{CDCl}_{3}\right)$. 


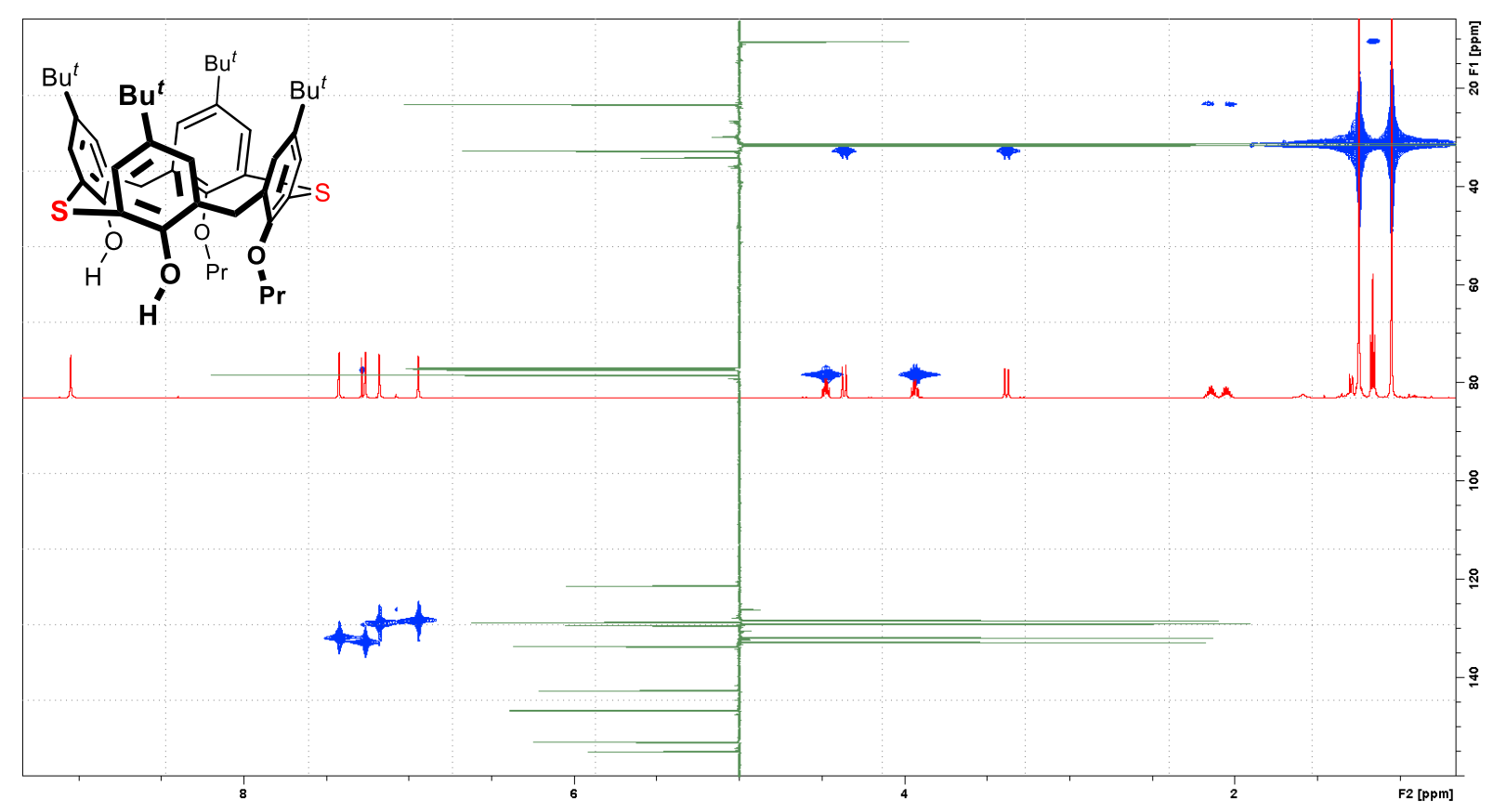

Figure S79. HMQC NMR of compound $\mathbf{8}\left(\mathrm{CDCl}_{3}\right)$.

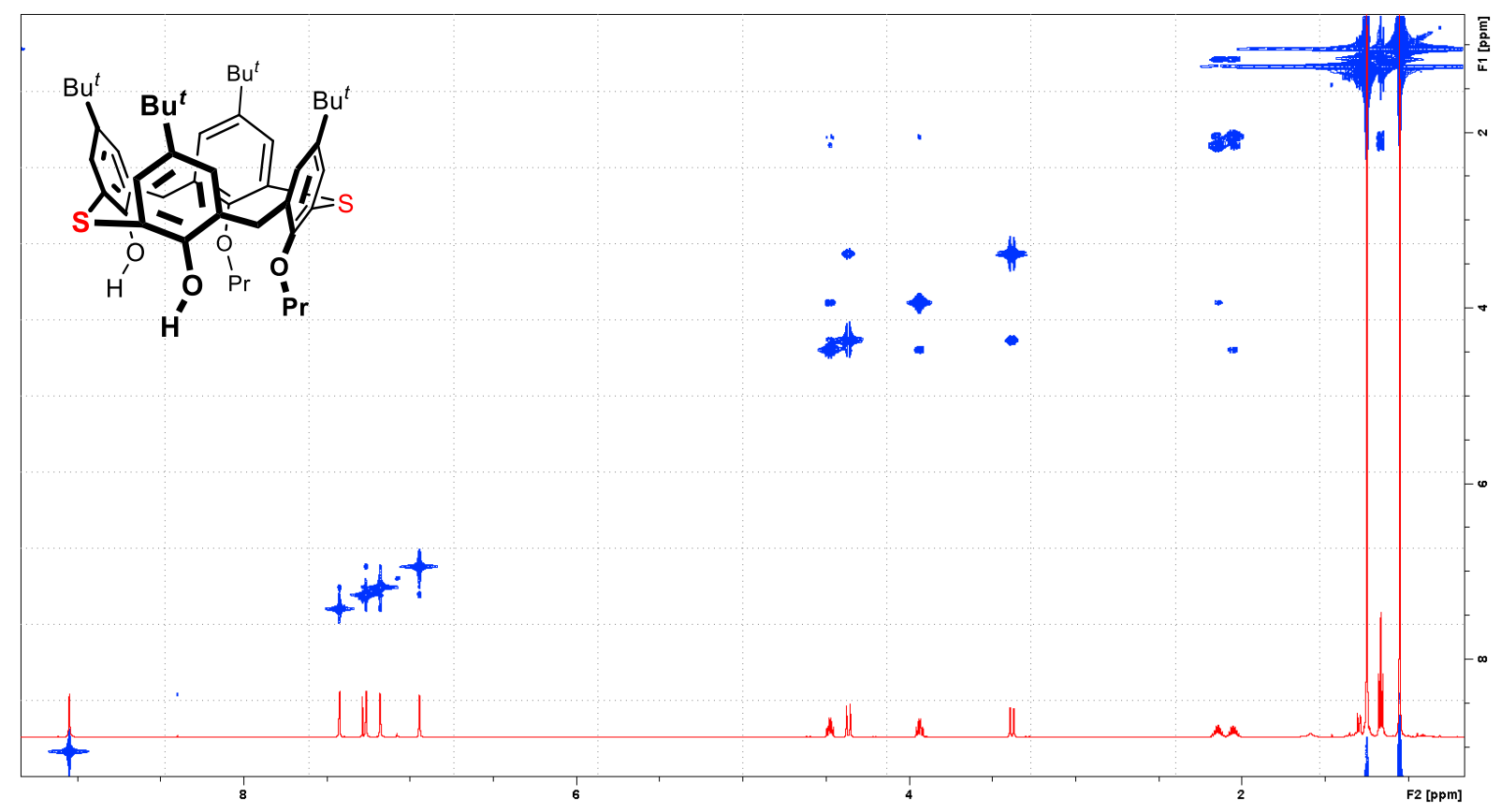

Figure S80. COSY NMR of compound $\mathbf{8}\left(\mathrm{CDCl}_{3}\right)$. 


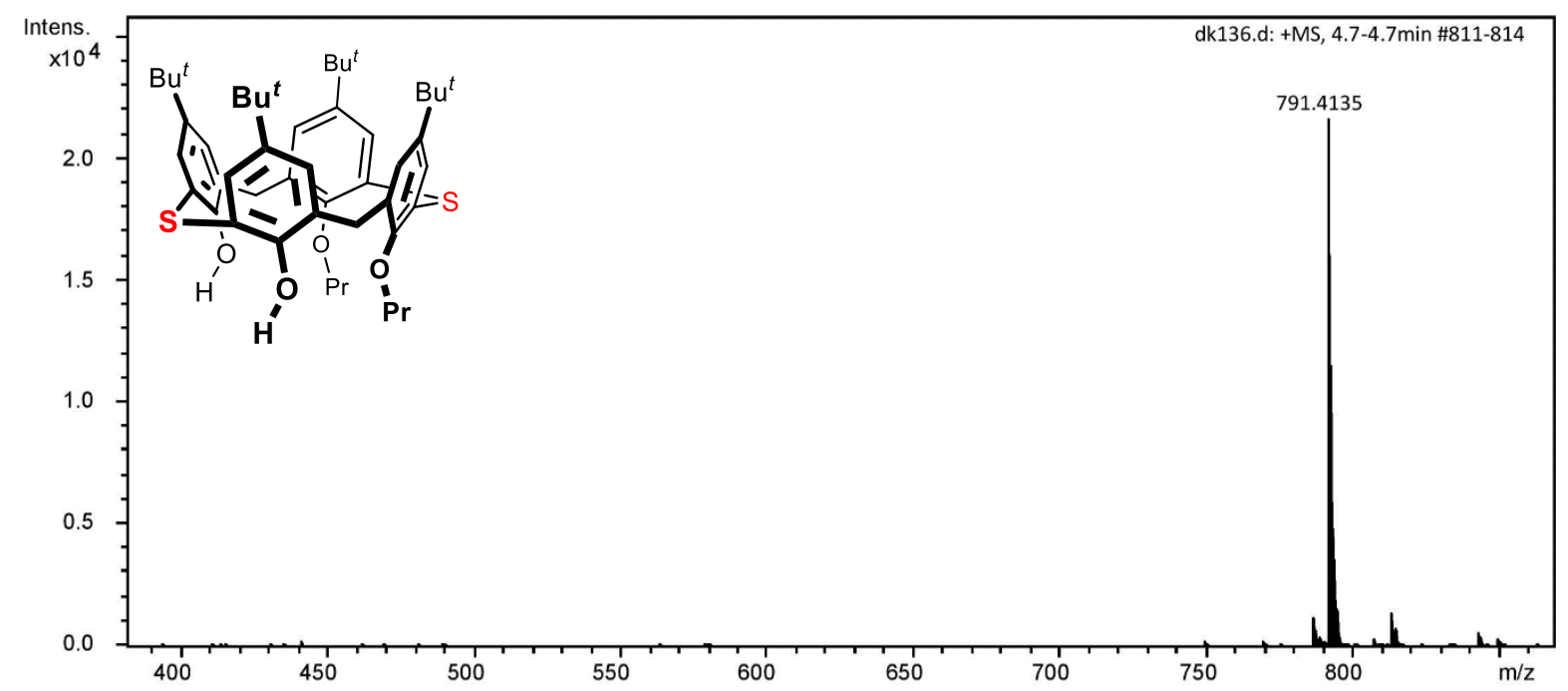

Figure S81. HRMS of compound $8\left(\mathrm{ESI}^{+}\right)$.

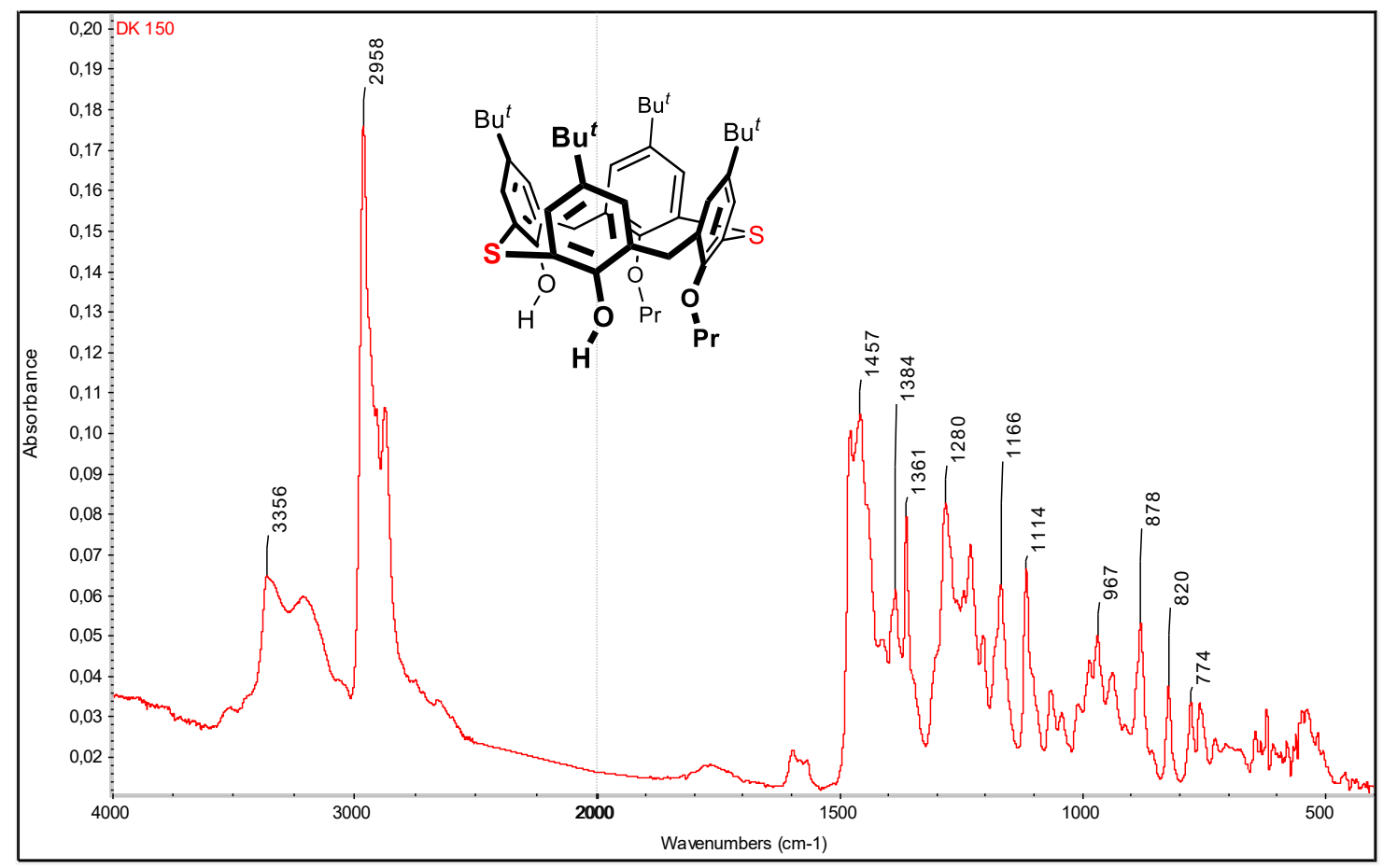

Figure S82. IR of compound 8 (ATR). 


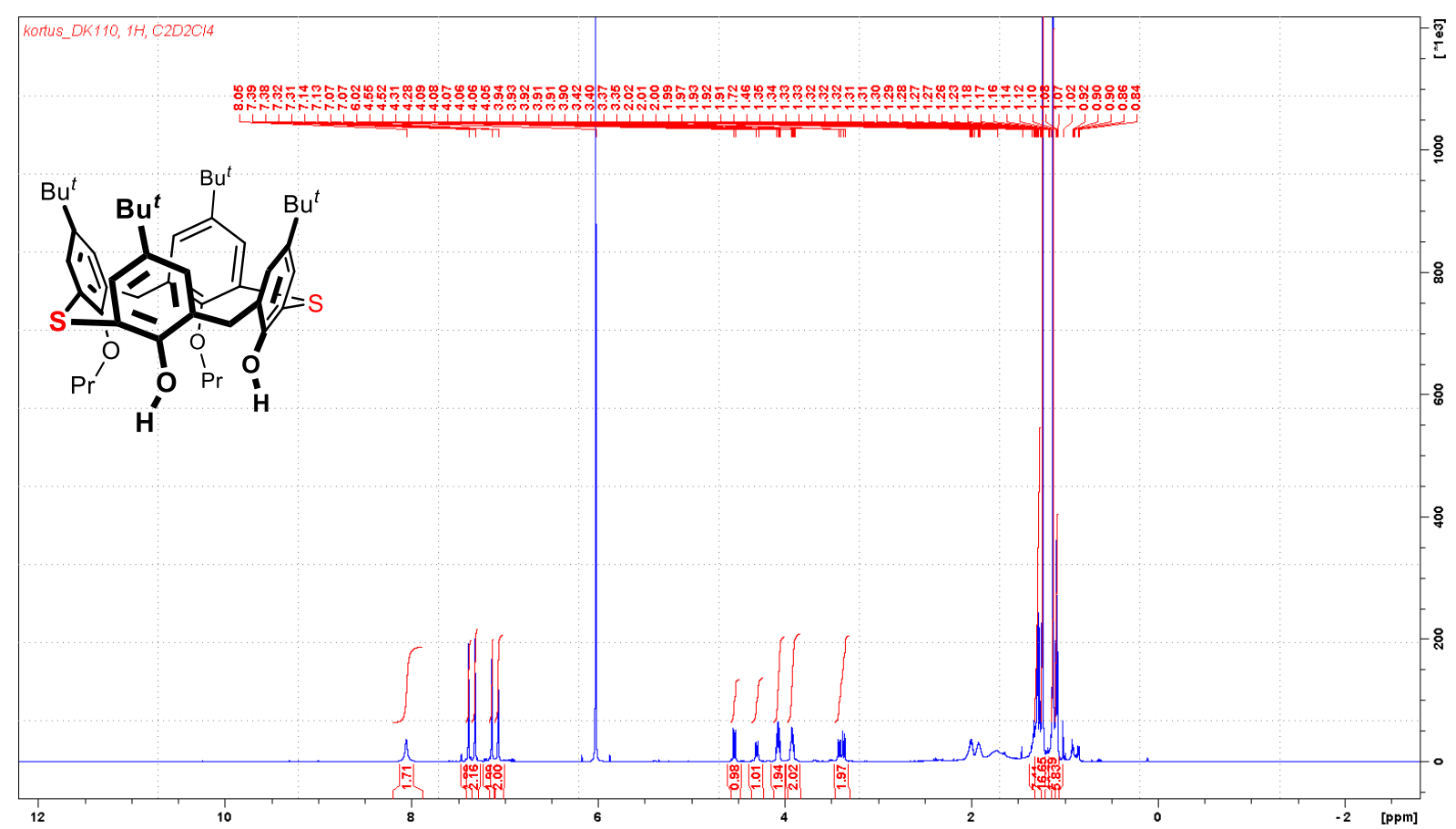

Figure S83. ${ }^{1} \mathrm{H}$ NMR of compound $9\left(\mathrm{C}_{2} \mathrm{D}_{2} \mathrm{Cl}_{4}, 600 \mathrm{MHz}\right)$.

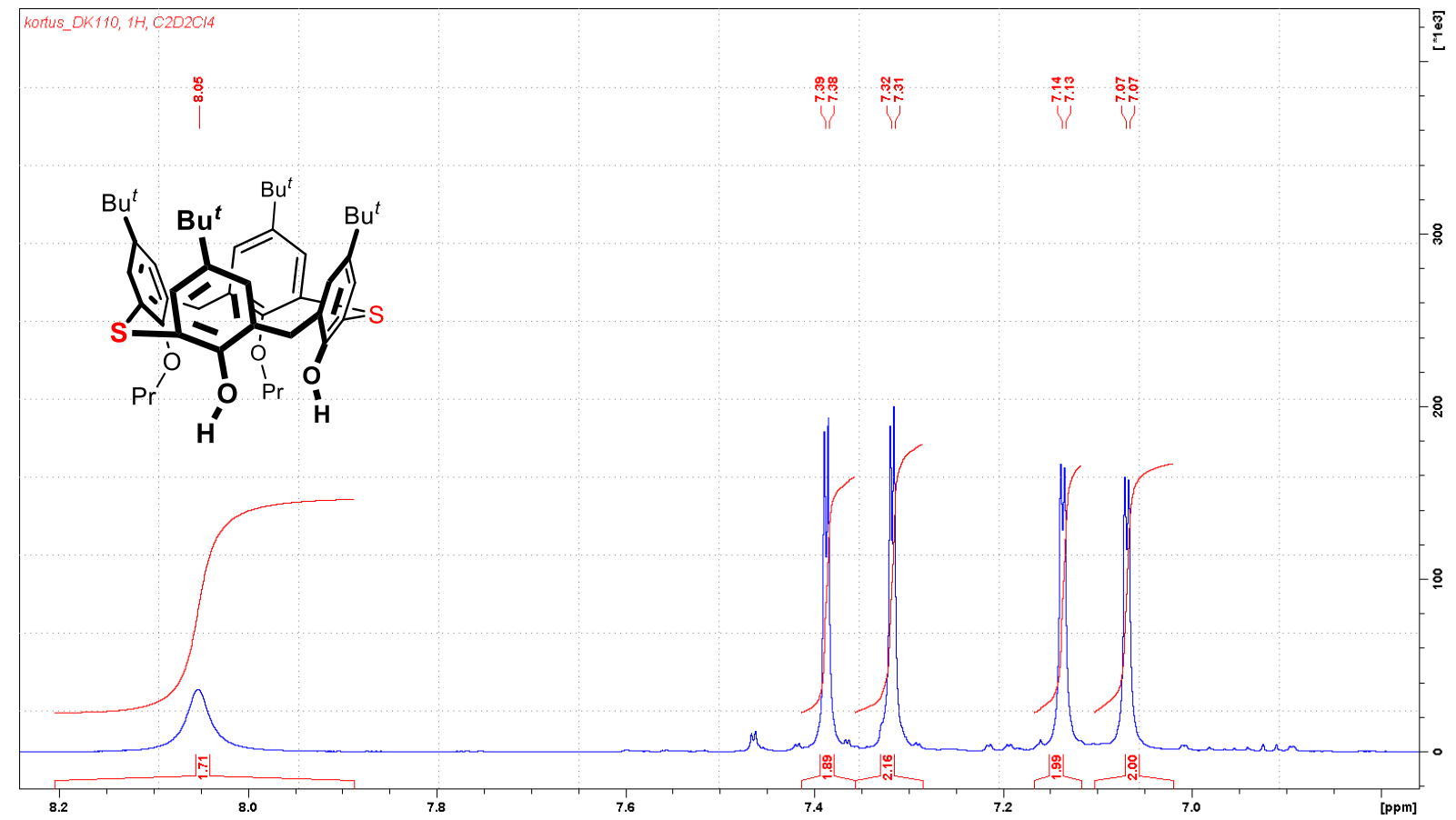

Figure S84. ${ }^{1} \mathrm{H}$ NMR of compound 9, aromatic region $\left(\mathrm{C}_{2} \mathrm{D}_{2} \mathrm{Cl}_{4}, 600 \mathrm{MHz}\right)$. 


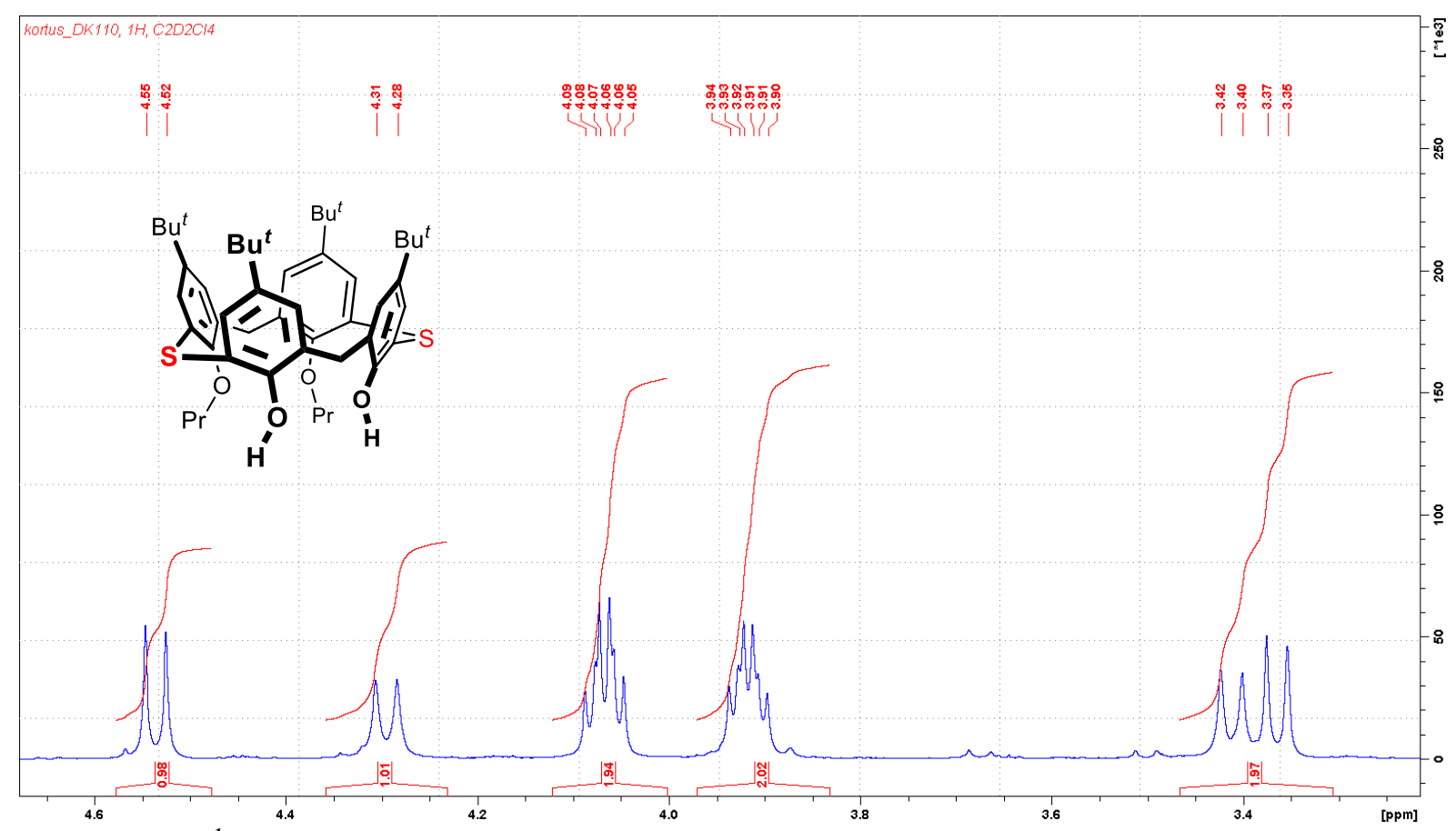

Figure S85. ${ }^{1} \mathrm{H}$ NMR of compound 9, bridge region $\left(\mathrm{C}_{2} \mathrm{D}_{2} \mathrm{Cl}_{4}, 600 \mathrm{MHz}\right)$.

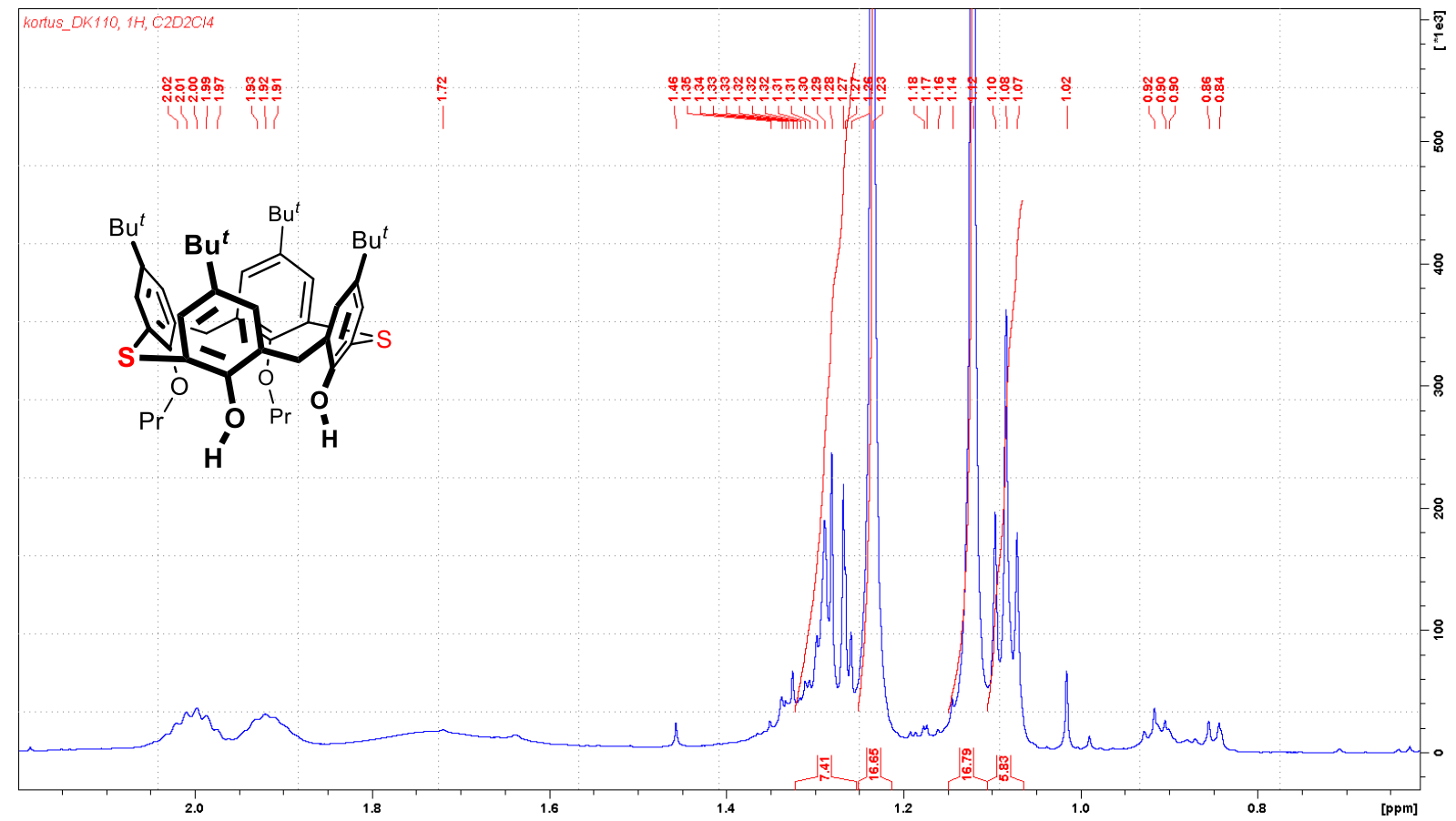

Figure S86. ${ }^{1} \mathrm{H}$ NMR of compound 9, aliphatic region $\left(\mathrm{C}_{2} \mathrm{D}_{2} \mathrm{Cl}_{4}, 600 \mathrm{MHz}\right)$. 


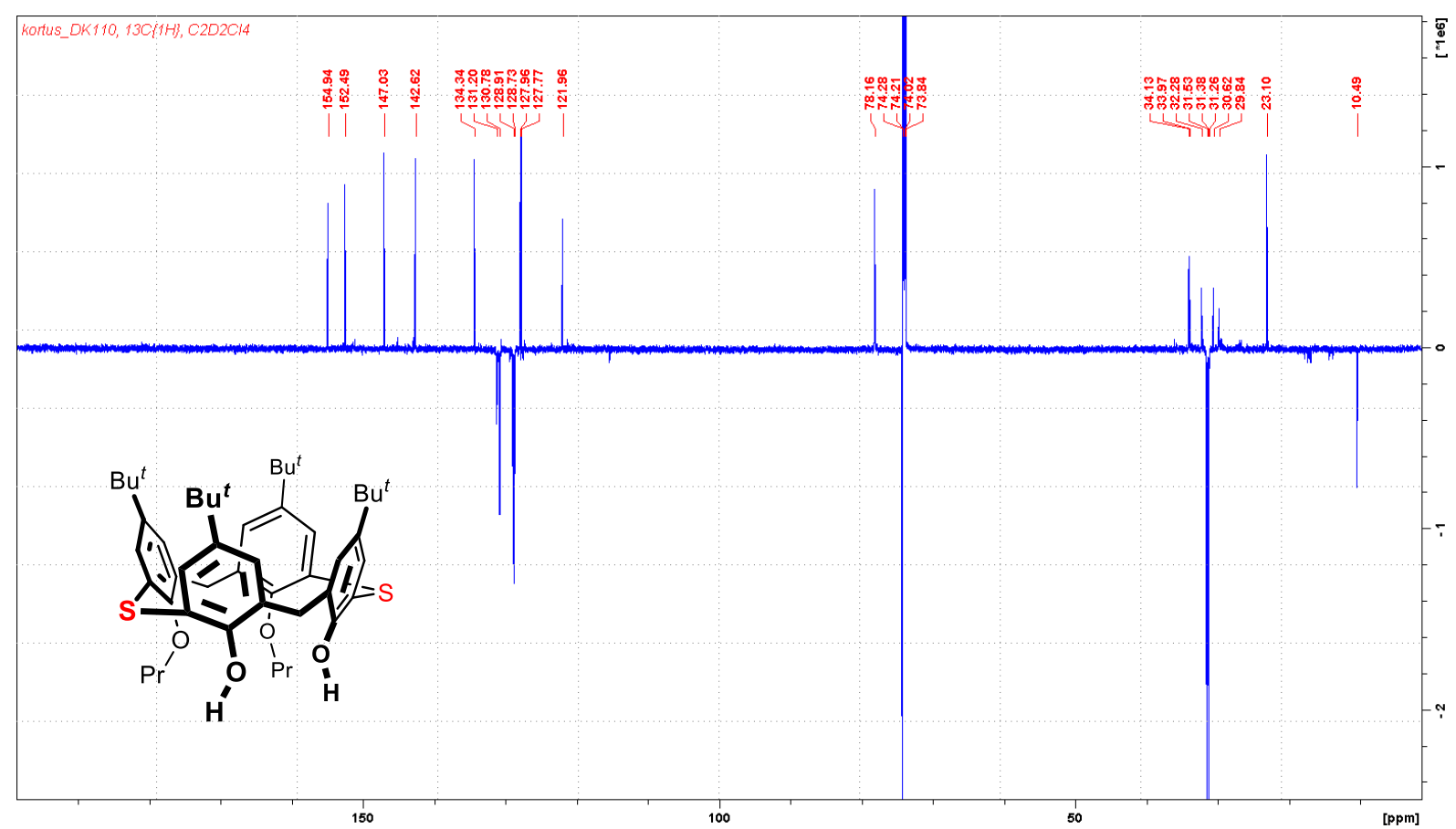

Figure S87. ${ }^{13} \mathrm{C}\left\{{ }^{1} \mathrm{H}\right\}$ NMR of compound $9\left(\mathrm{C}_{2} \mathrm{D}_{2} \mathrm{Cl}_{4}, 151 \mathrm{MHz}\right)$.

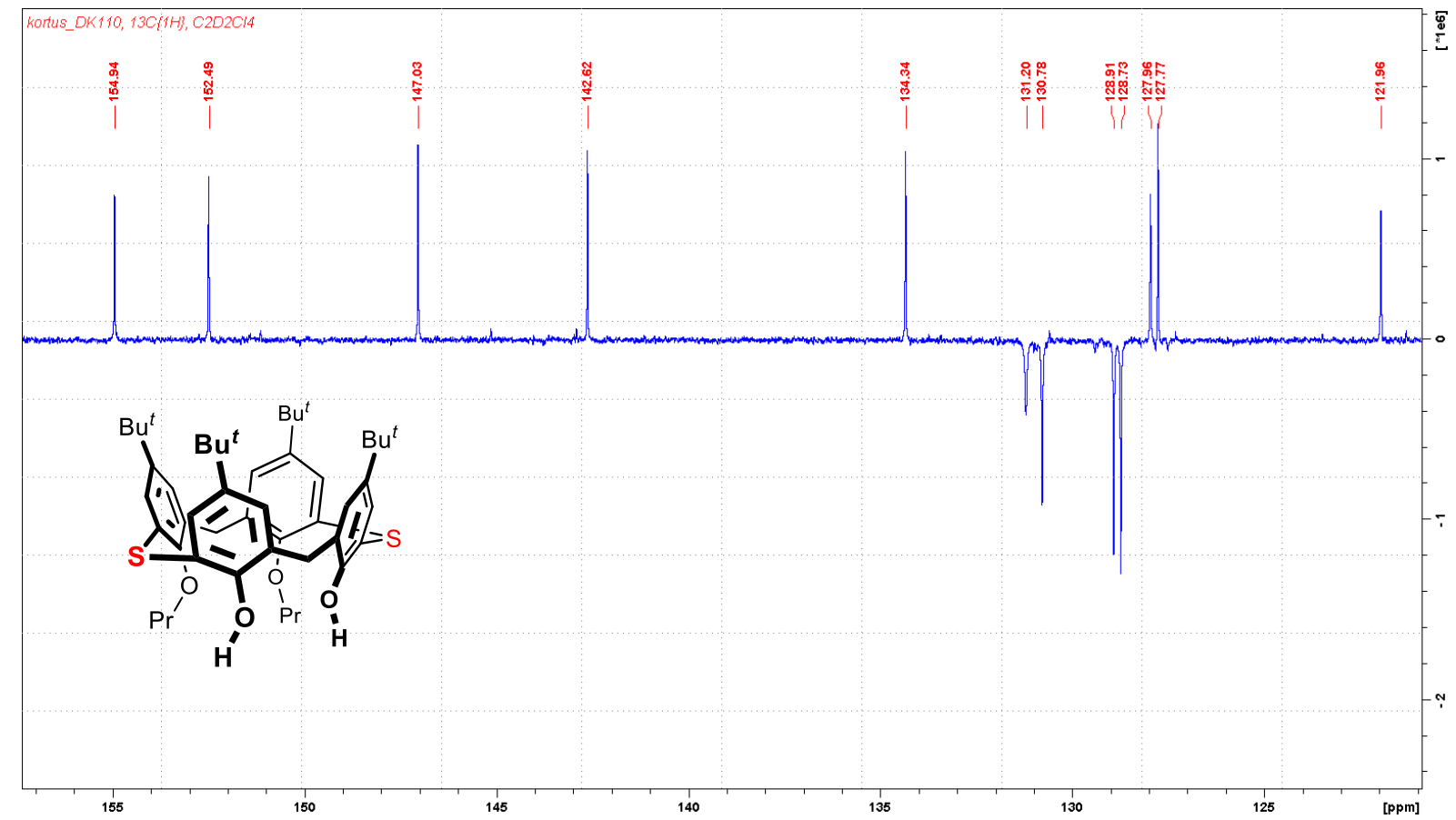

Figure S88. ${ }^{13} \mathrm{C}\left\{{ }^{1} \mathrm{H}\right\}$ NMR of compound 9, aromatic region $\left(\mathrm{C}_{2} \mathrm{D}_{2} \mathrm{Cl}_{4}, 151 \mathrm{MHz}\right)$. 


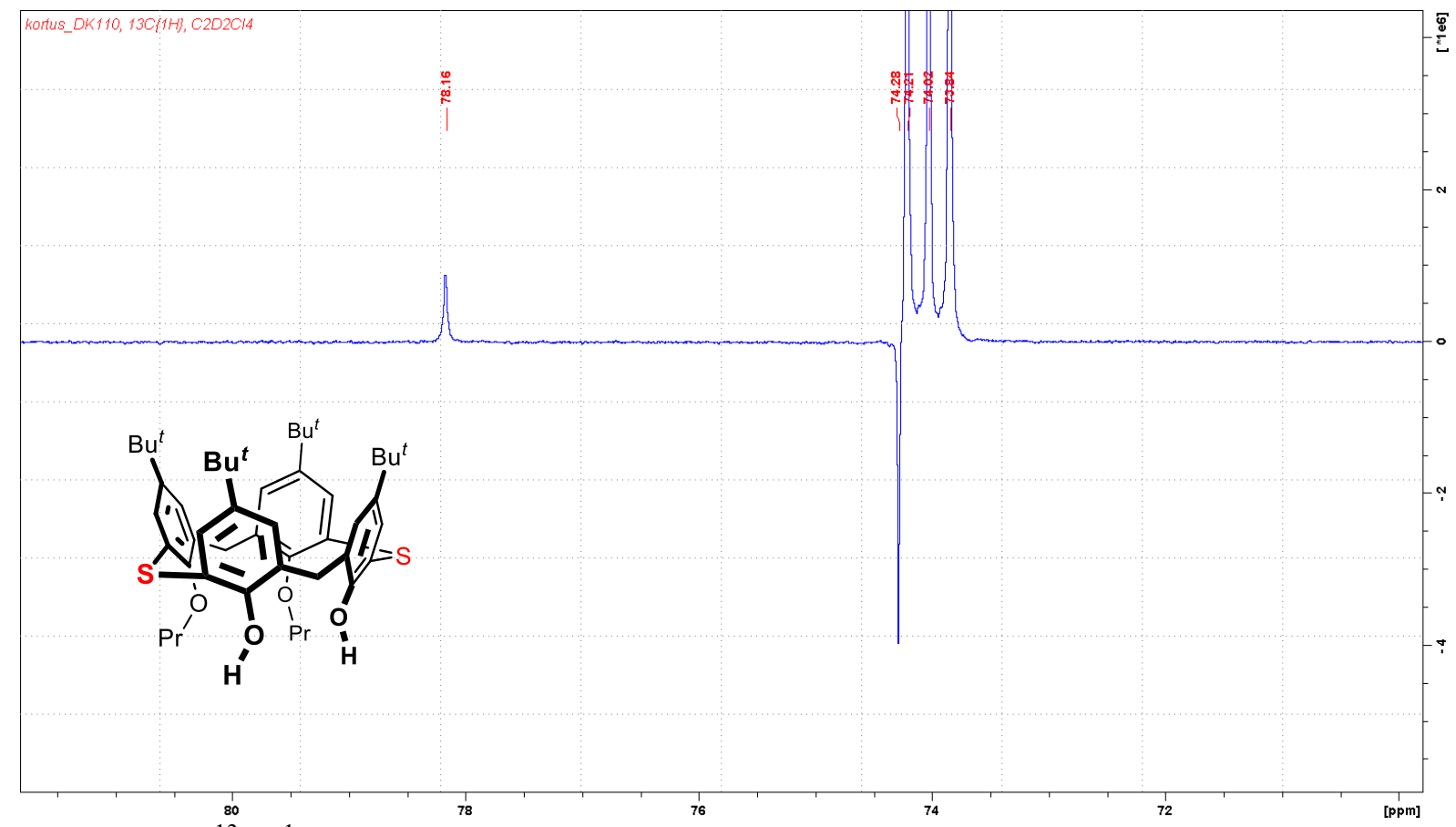

Figure S89. ${ }^{13} \mathrm{C}\left\{{ }^{1} \mathrm{H}\right\}$ NMR of compound 9, aliphatic region $\left(\mathrm{C}_{2} \mathrm{D}_{2} \mathrm{Cl}_{4}, 151 \mathrm{MHz}\right)$.

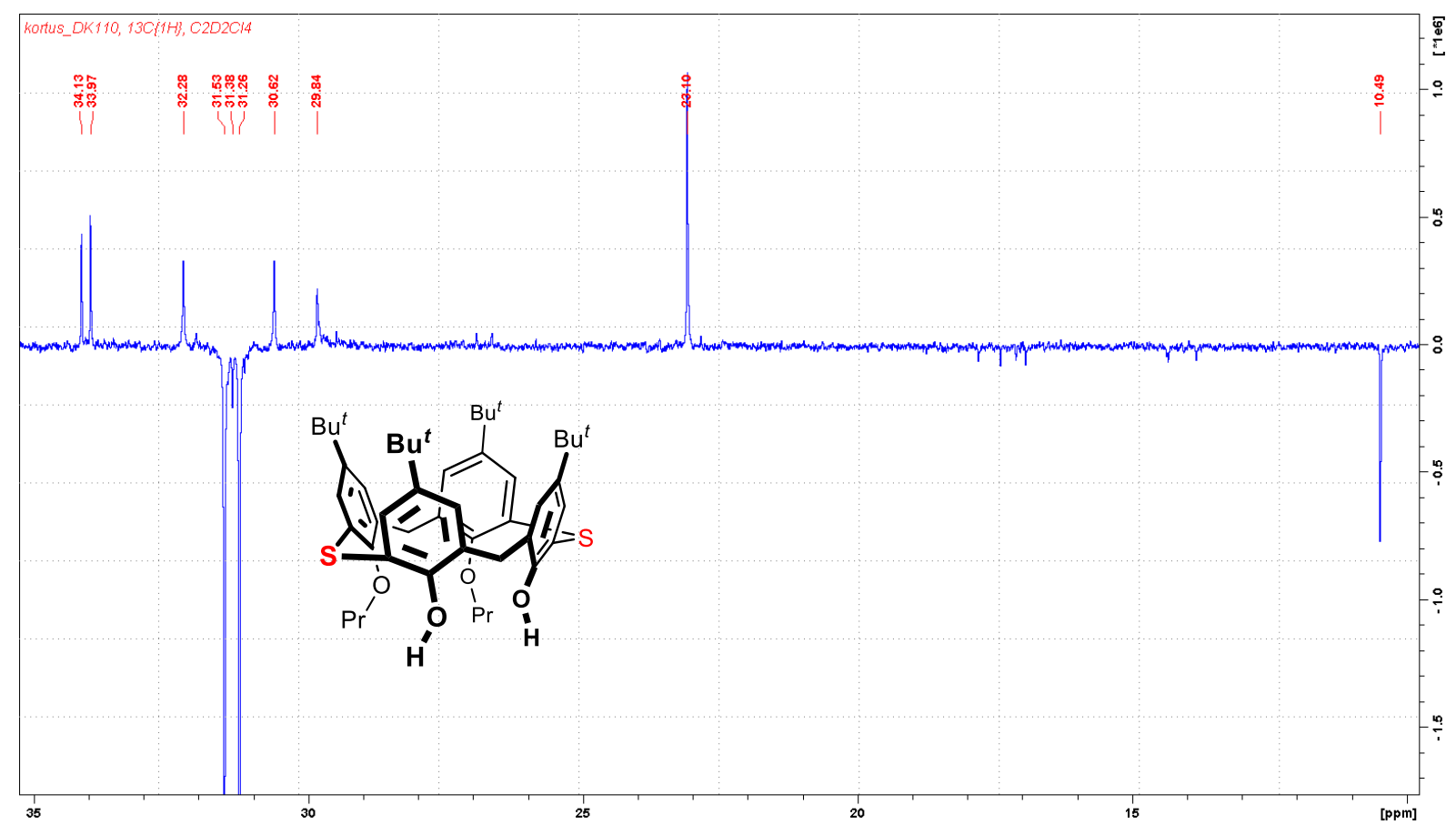

Figure S90. ${ }^{13} \mathrm{C}\left\{{ }^{1} \mathrm{H}\right\}$ NMR of compound 9, aliphatic region $\left(\mathrm{C}_{2} \mathrm{D}_{2} \mathrm{Cl}_{4}, 151 \mathrm{MHz}\right)$. 


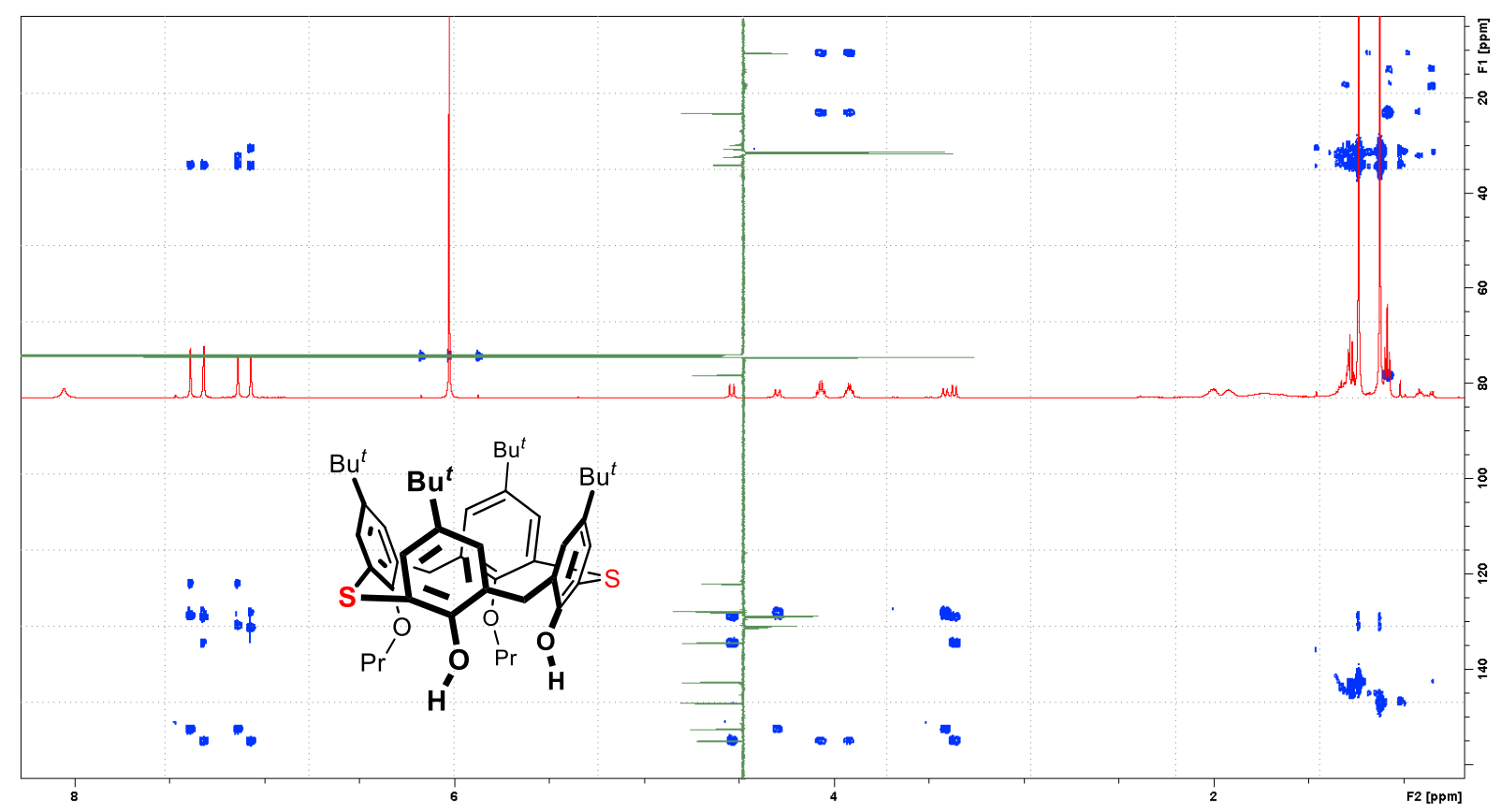

Figure S91. HMBC NMR of compound $9\left(\mathrm{C}_{2} \mathrm{D}_{2} \mathrm{Cl}_{4}\right)$.

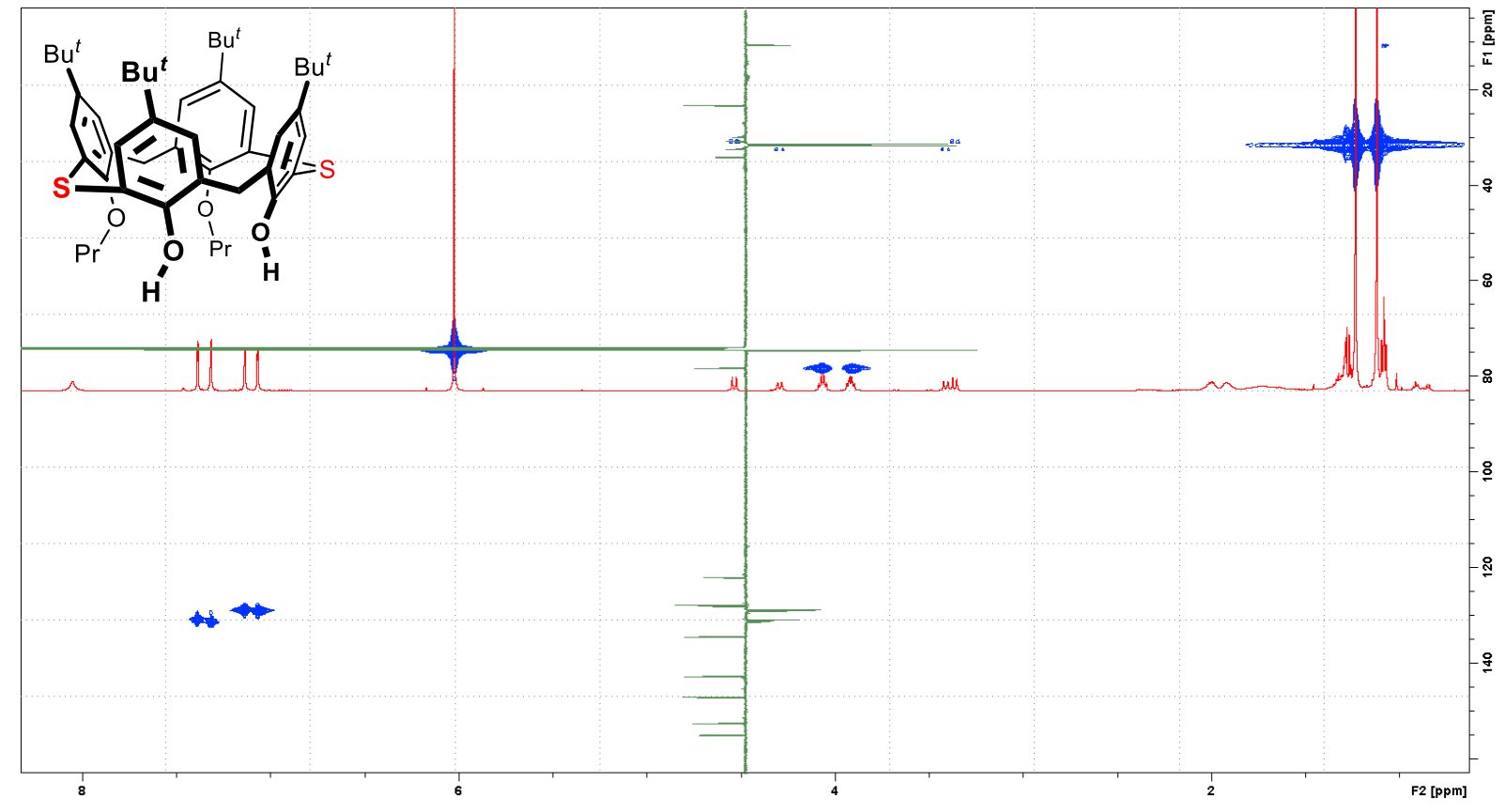

Figure S92. HMQC NMR of compound $9\left(\mathrm{C}_{2} \mathrm{D}_{2} \mathrm{Cl}_{4}\right)$. 


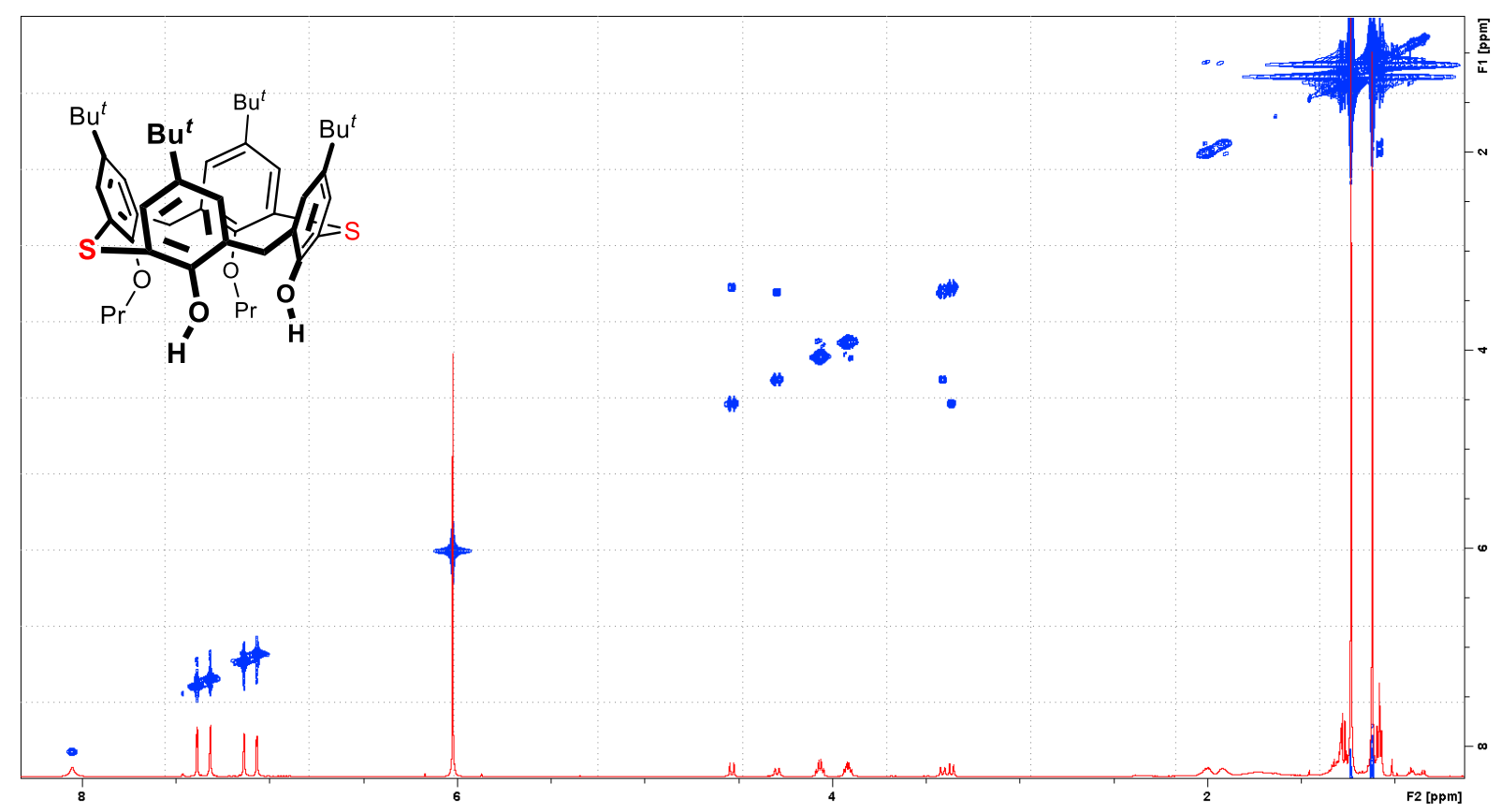

Figure S93. COSY NMR of compound $9\left(\mathrm{C}_{2} \mathrm{D}_{2} \mathrm{Cl}_{4}\right)$.

calc for $\left[\mathrm{C}_{48} \mathrm{H}_{64} \mathrm{O}_{4} \mathrm{~S}_{2} \mathrm{Na}\right]+791.4138[\mathrm{M}+\mathrm{Na}]+$

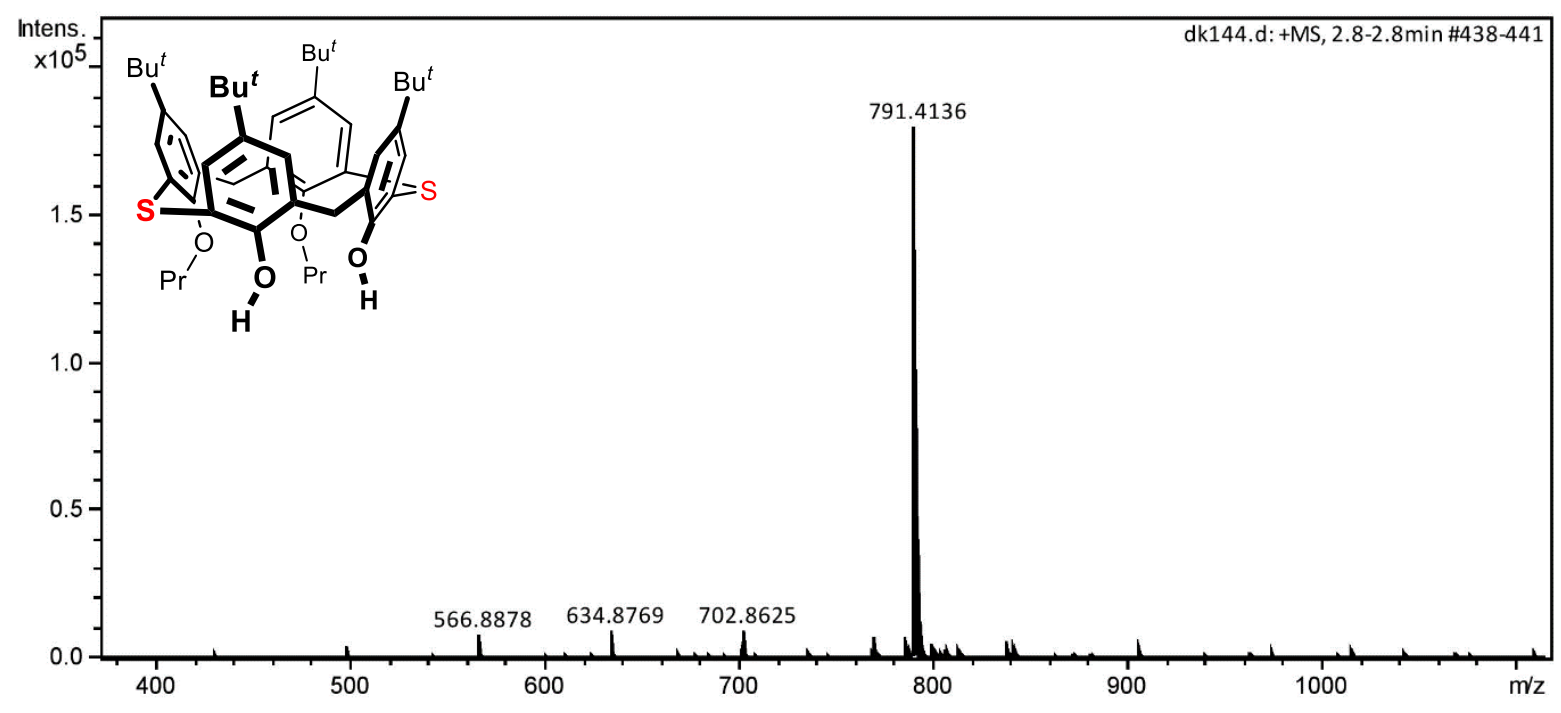

Figure S94. HRMS of compound $9\left(\mathrm{ESI}^{+}\right)$. 


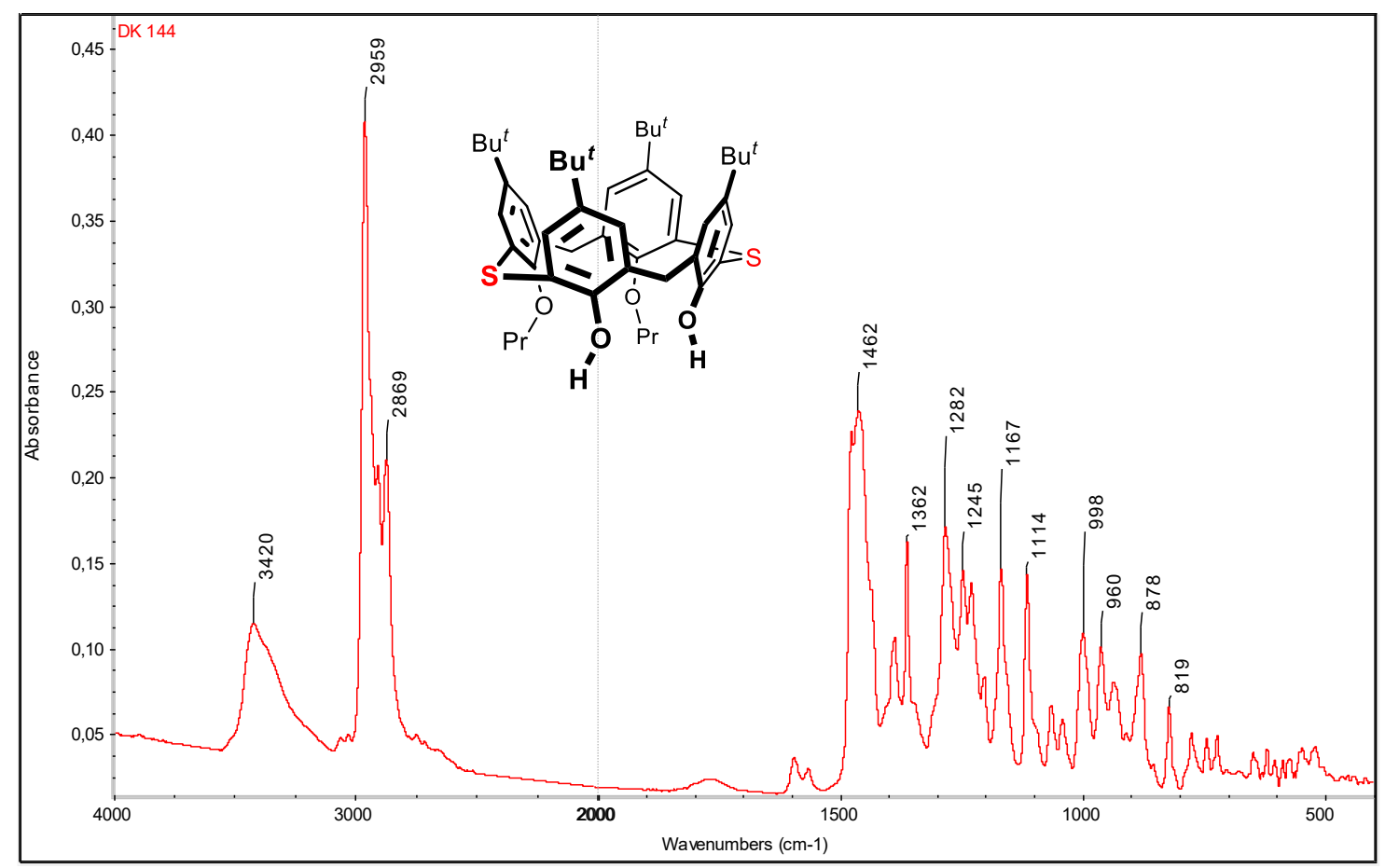

Figure S95. IR of compound 9 (ATR). 


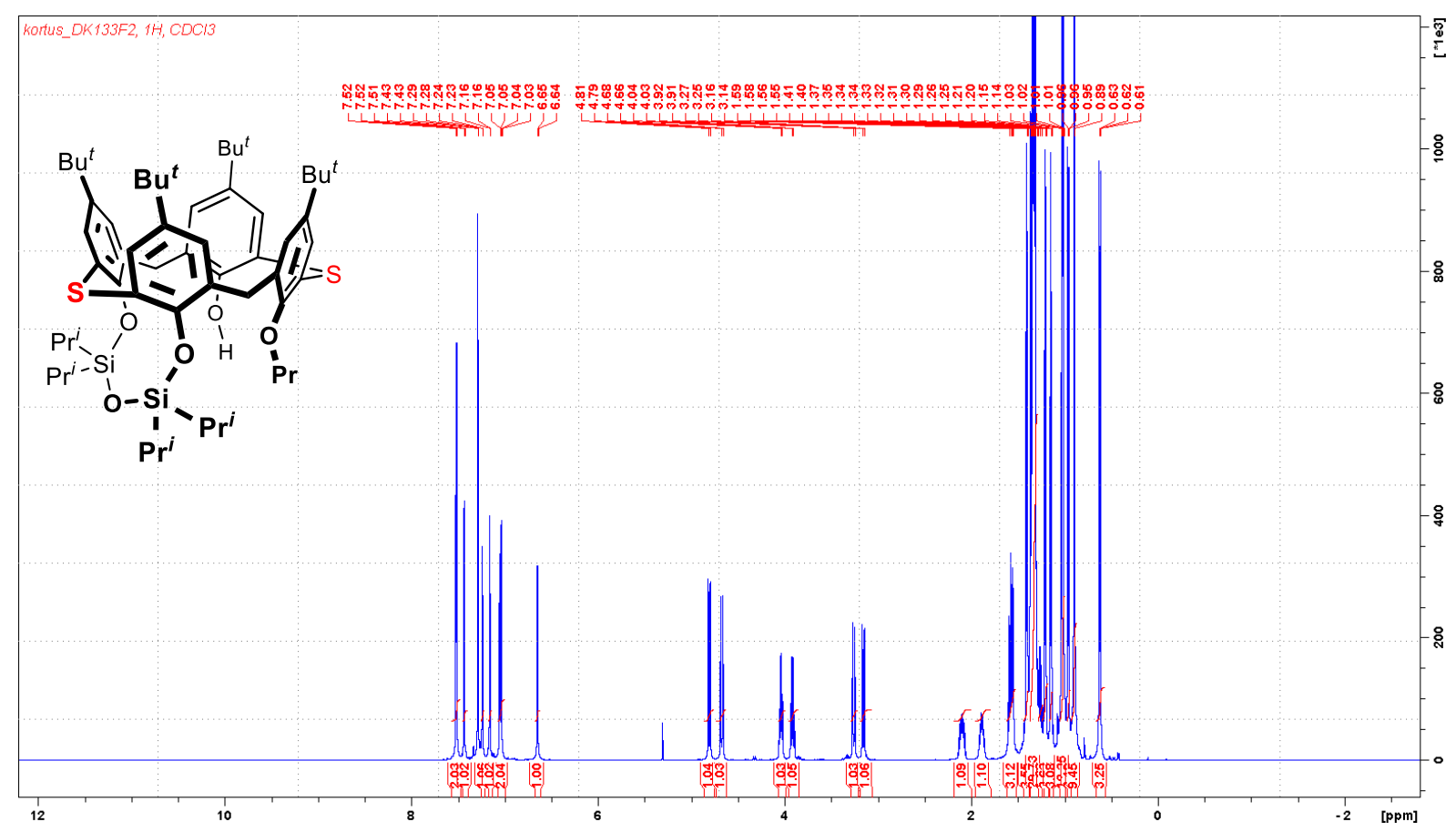

Figure S96. ${ }^{1} \mathrm{H}$ NMR of compound $10\left(\mathrm{CDCl}_{3}, 600 \mathrm{MHz}\right)$.

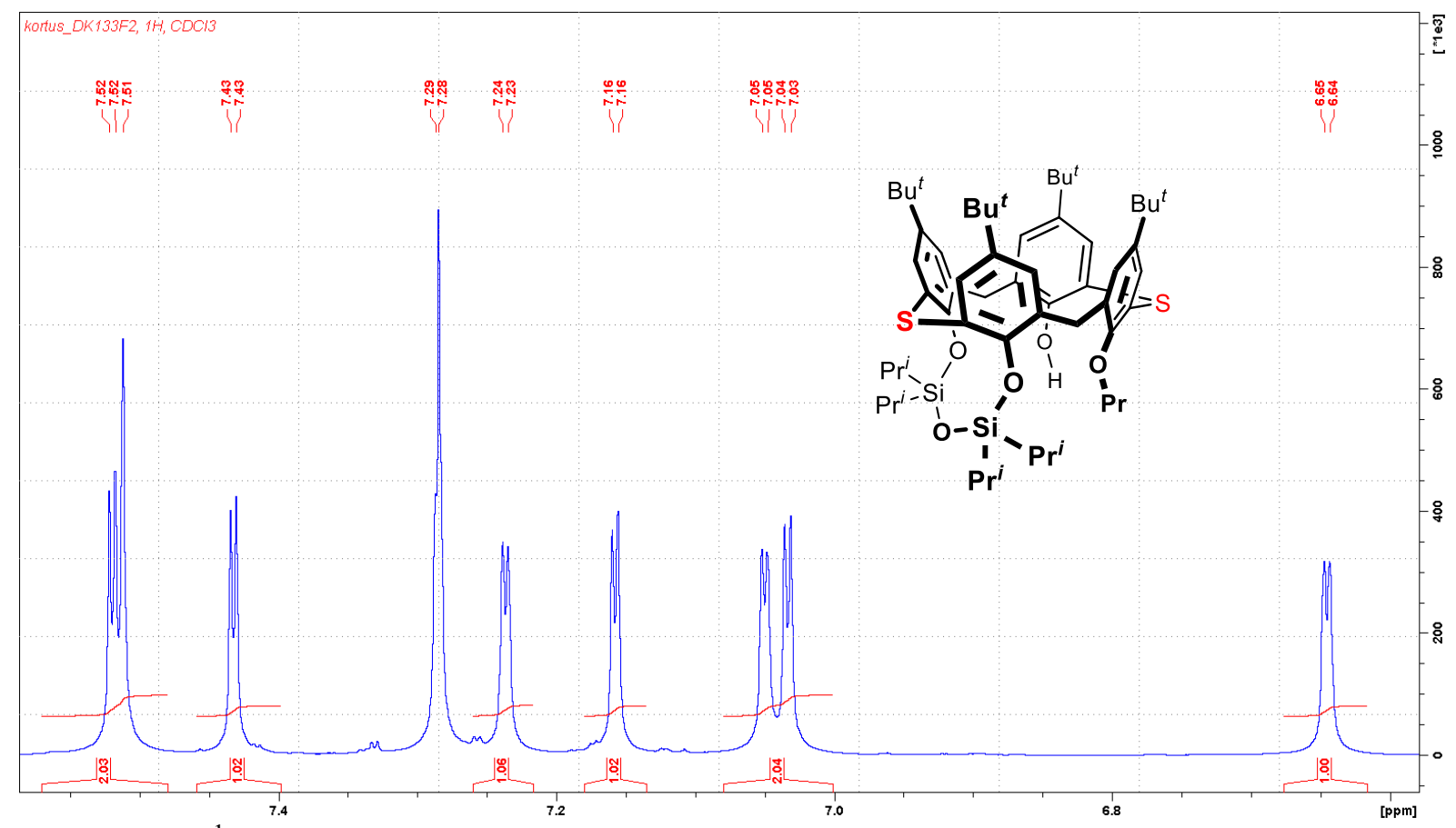

Figure S97. ${ }^{1} \mathrm{H}$ NMR of compound 10, aromatic region $\left(\mathrm{CDCl}_{3}, 600 \mathrm{MHz}\right)$. 


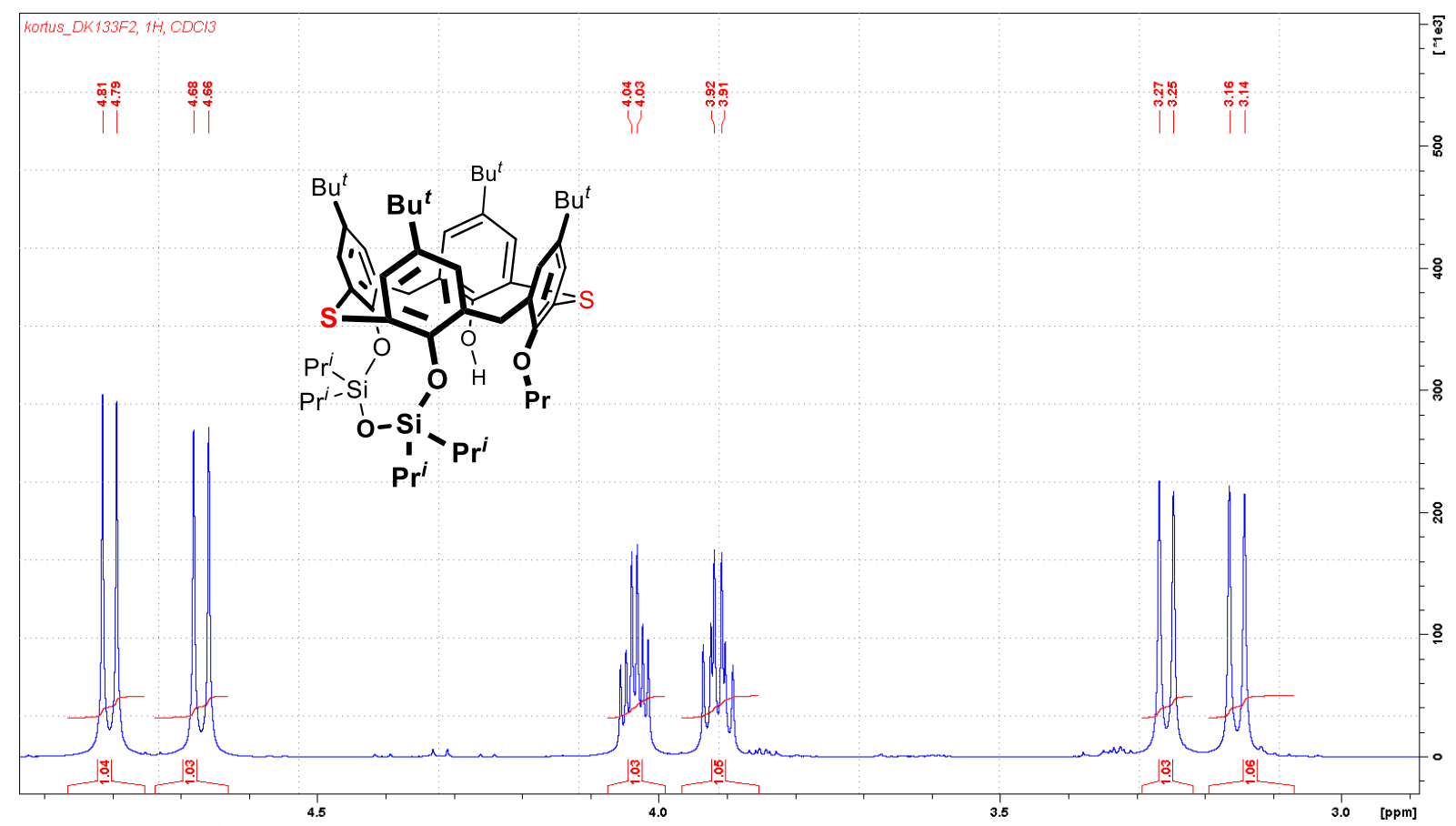

Figure S98. ${ }^{1} \mathrm{H}$ NMR of compound 10, bridge region $\left(\mathrm{CDCl}_{3}, 600 \mathrm{MHz}\right)$.

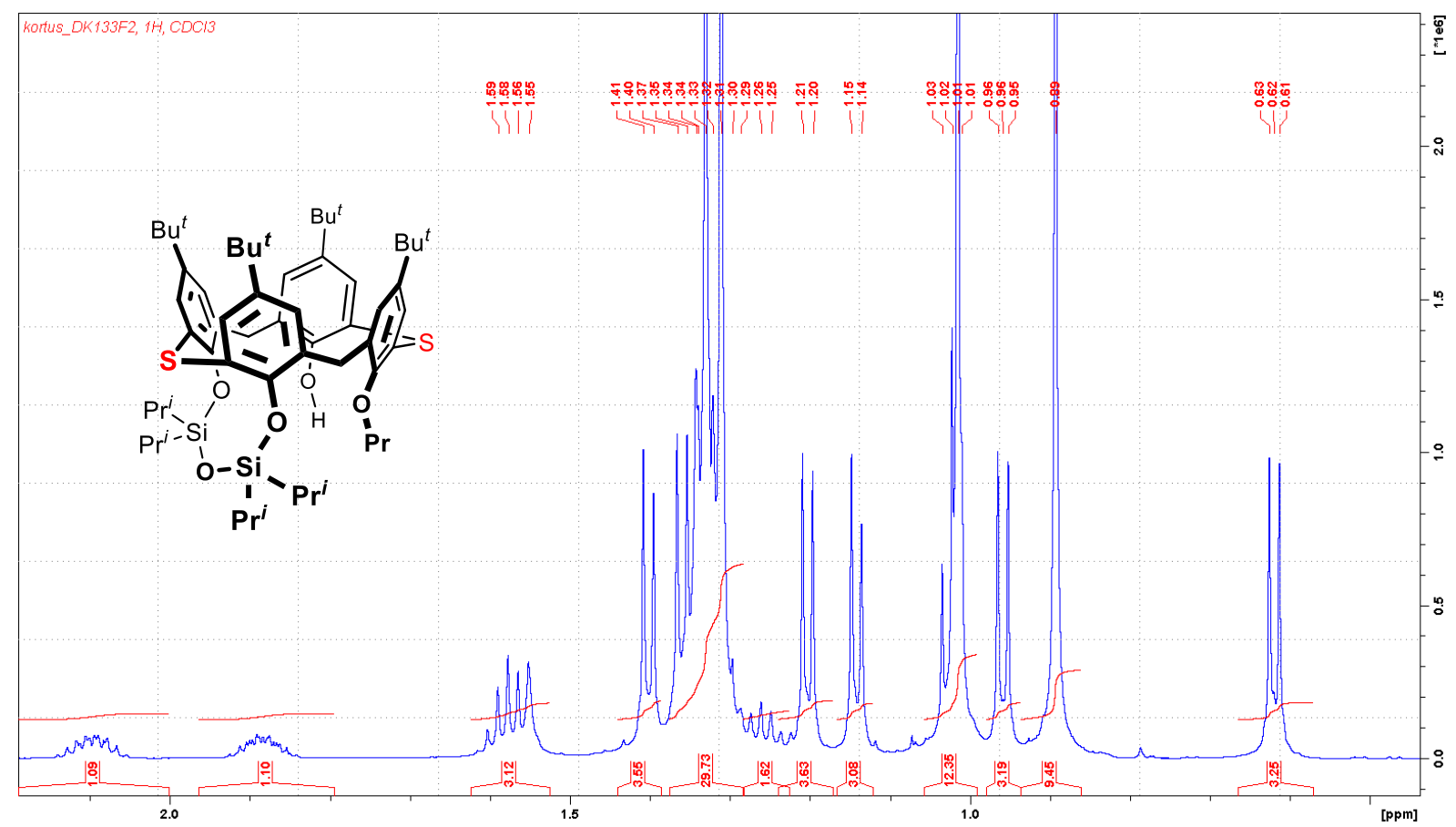

Figure S99. ${ }^{1} \mathrm{H}$ NMR of compound 10, aliphatic region $\left(\mathrm{CDCl}_{3}, 600 \mathrm{MHz}\right)$. 


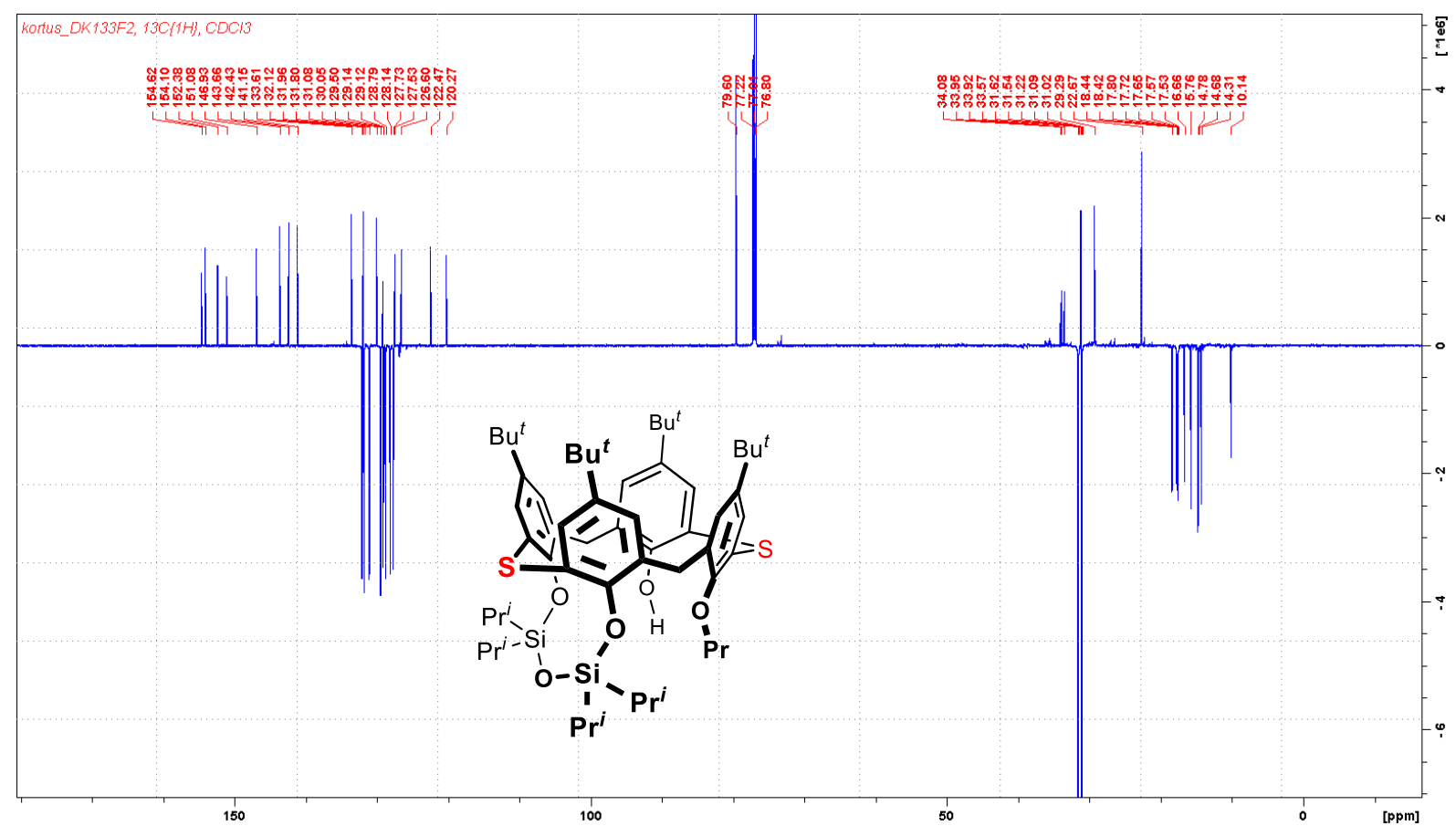

Figure S100. ${ }^{13} \mathrm{C}\left\{{ }^{1} \mathrm{H}\right\}$ NMR of compound $\mathbf{1 0}\left(\mathrm{C}_{2} \mathrm{D}_{2} \mathrm{Cl}_{4}, 151 \mathrm{MHz}\right)$.

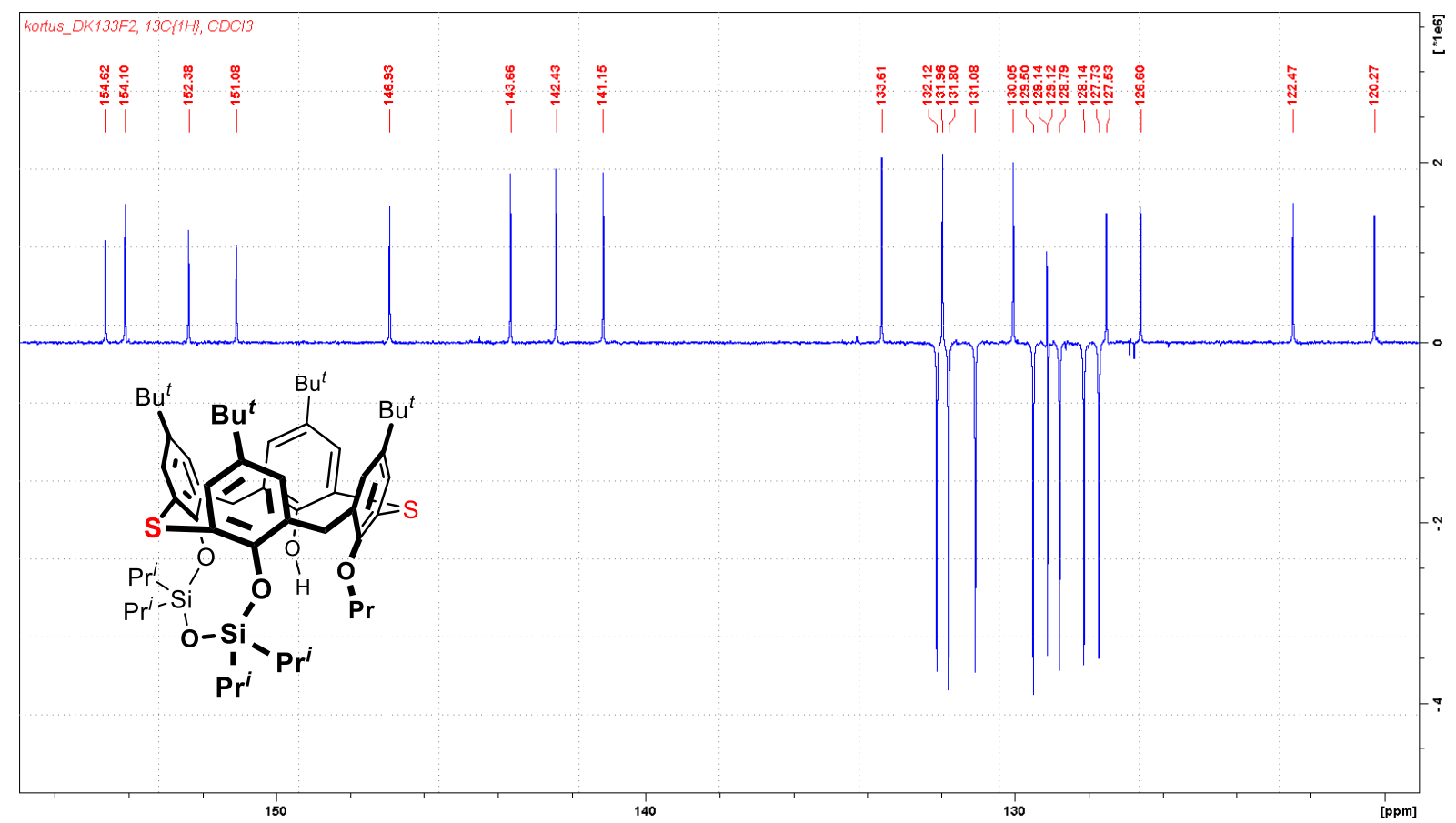

Figure S101. ${ }^{13} \mathrm{C}\left\{{ }^{1} \mathrm{H}\right\}$ NMR of compound 10, aromatic region $\left(\mathrm{C}_{2} \mathrm{D}_{2} \mathrm{Cl}_{4}, 151 \mathrm{MHz}\right)$. 


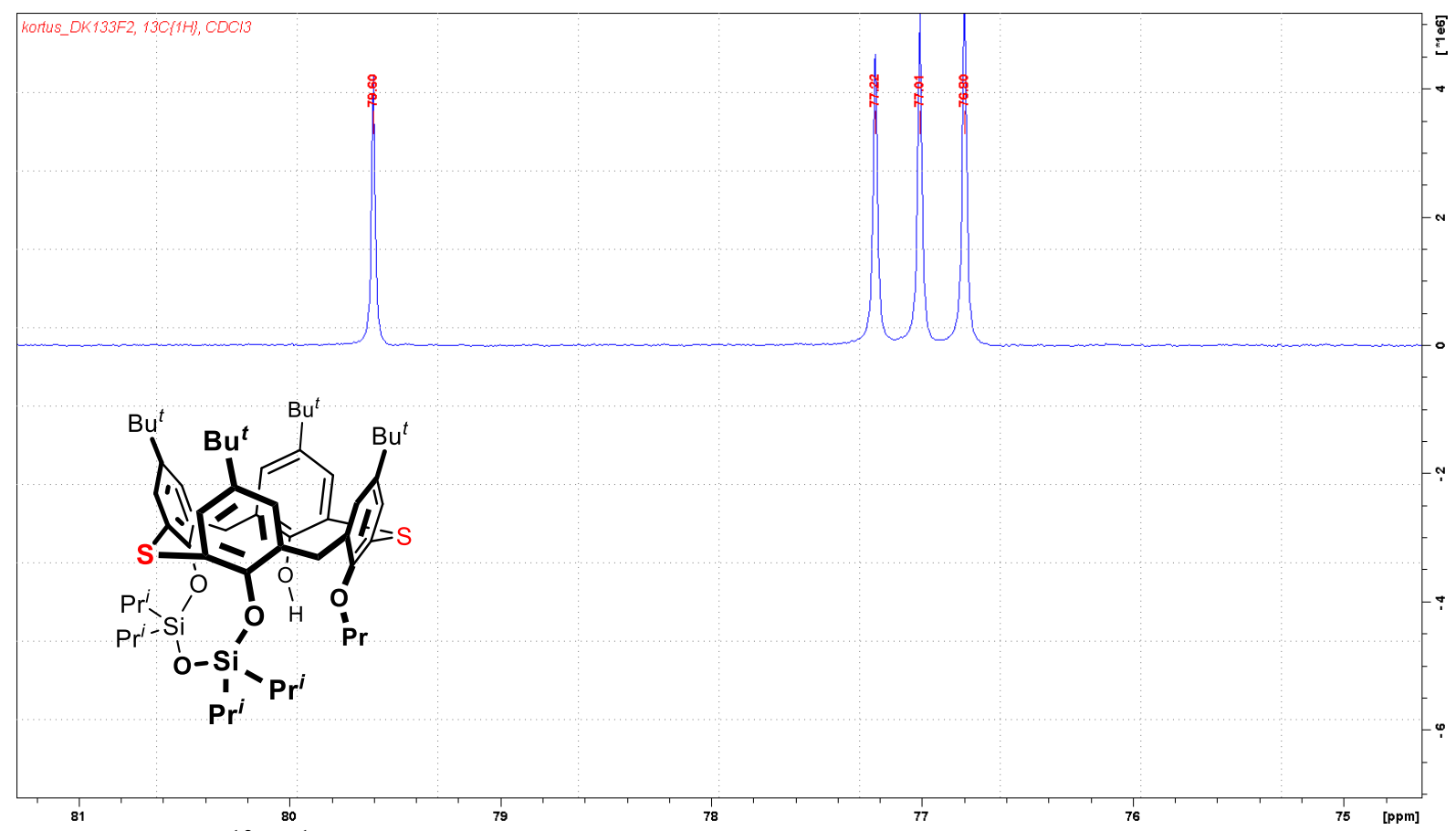

Figure S102. ${ }^{13} \mathrm{C}\left\{{ }^{1} \mathrm{H}\right\}$ NMR of compound 10, aliphatic region $\left(\mathrm{C}_{2} \mathrm{D}_{2} \mathrm{Cl} \mathrm{l}_{4}, 151 \mathrm{MHz}\right)$.

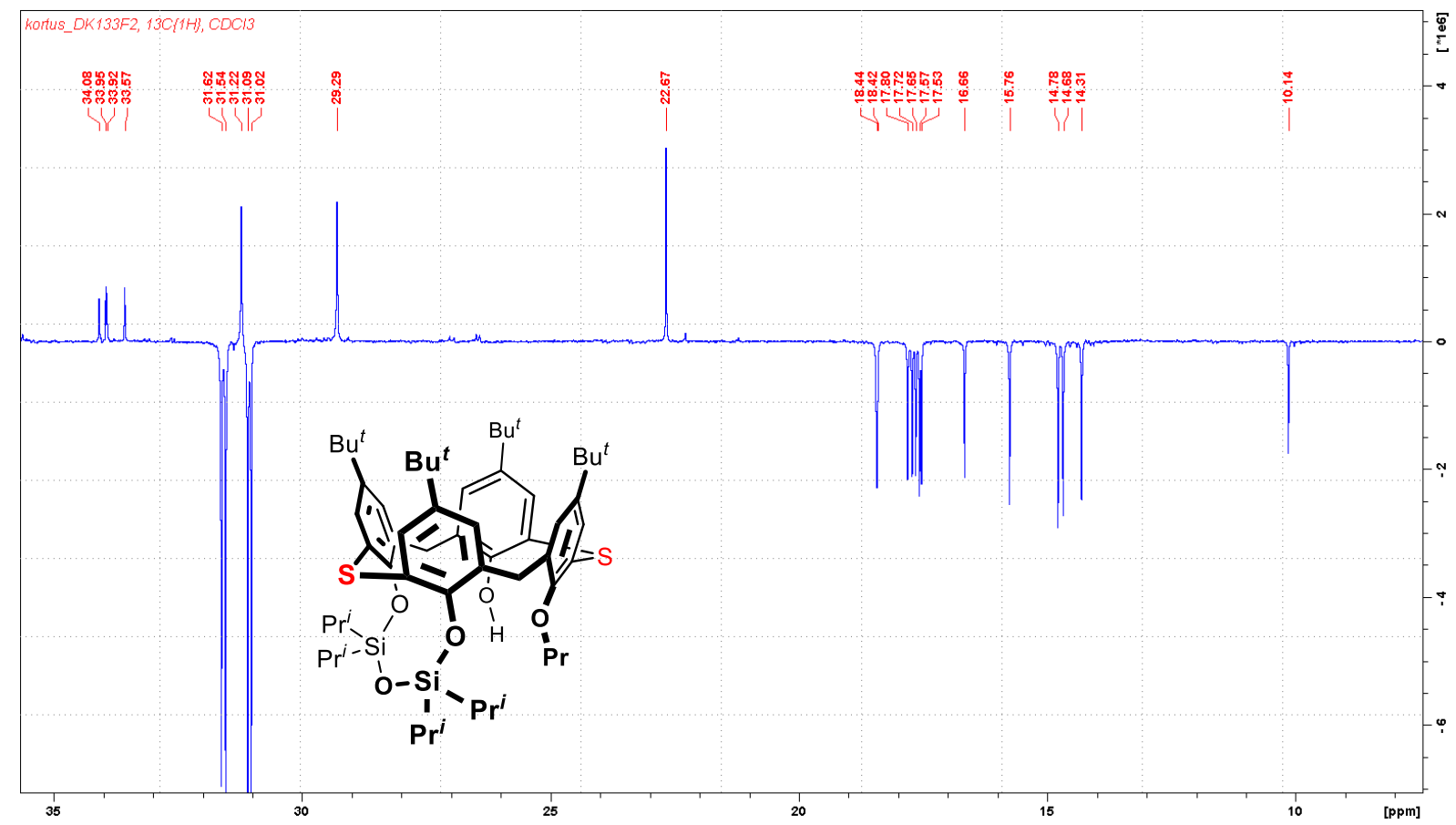

Figure S103. ${ }^{13} \mathrm{C}\left\{{ }^{1} \mathrm{H}\right\}$ NMR of compound 10, aliphatic region $\left(\mathrm{C}_{2} \mathrm{D}_{2} \mathrm{Cl}_{4}, 151 \mathrm{MHz}\right)$. 


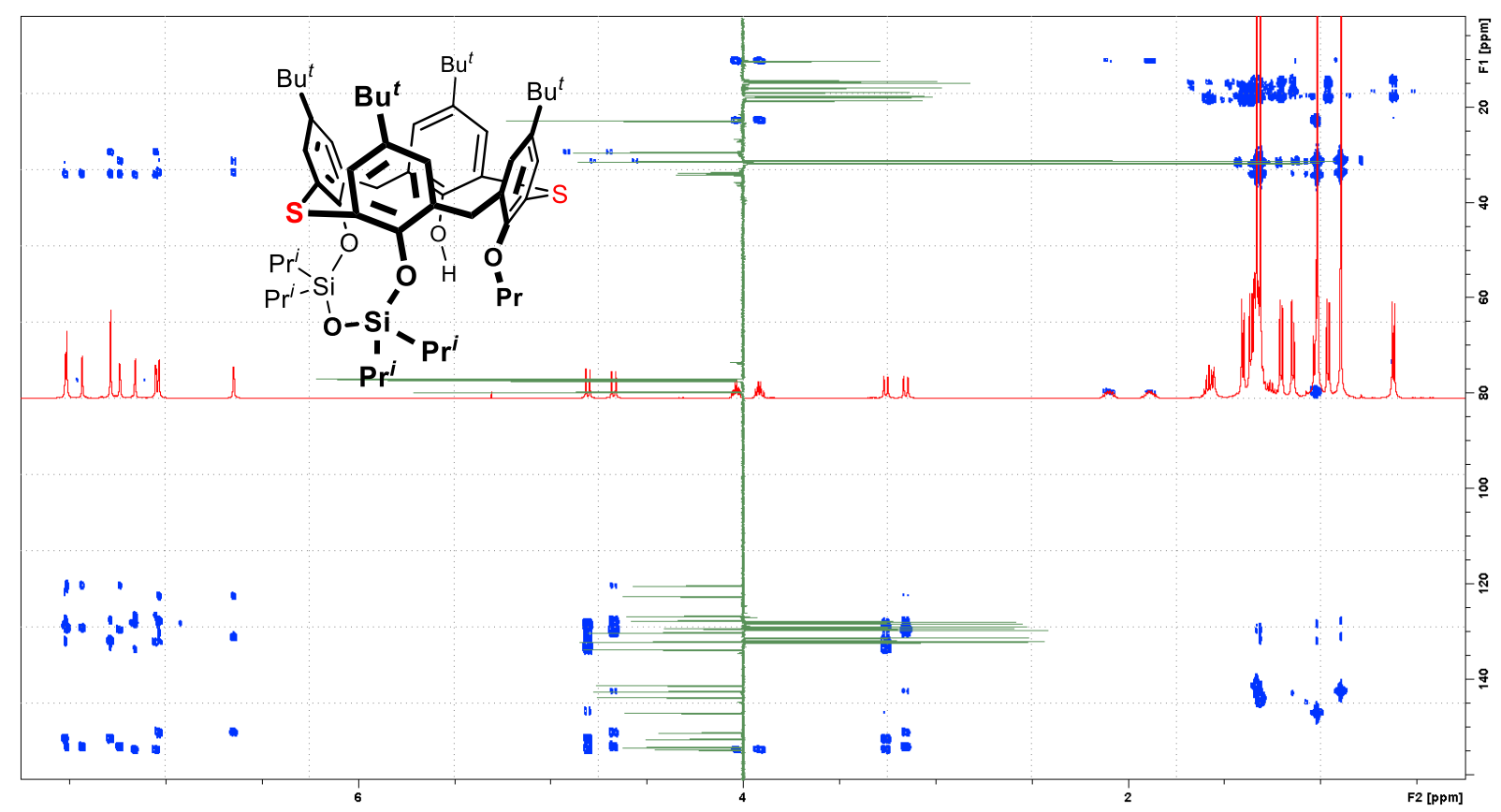

Figure S104. HMBC NMR of compound $10\left(\mathrm{CDCl}_{3}\right)$.

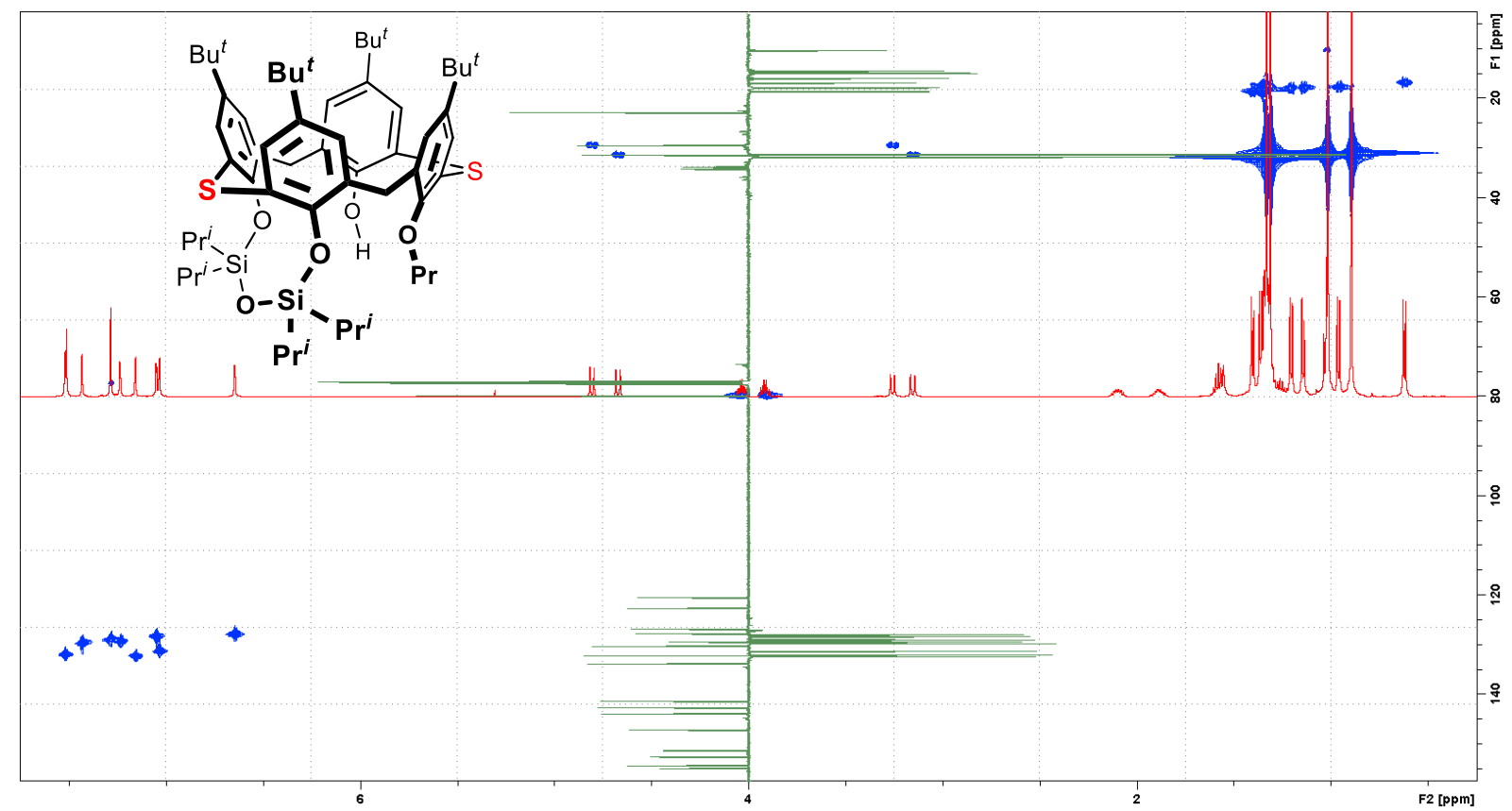

Figure S105. HMQC NMR of compound $10\left(\mathrm{CDCl}_{3}\right)$. 


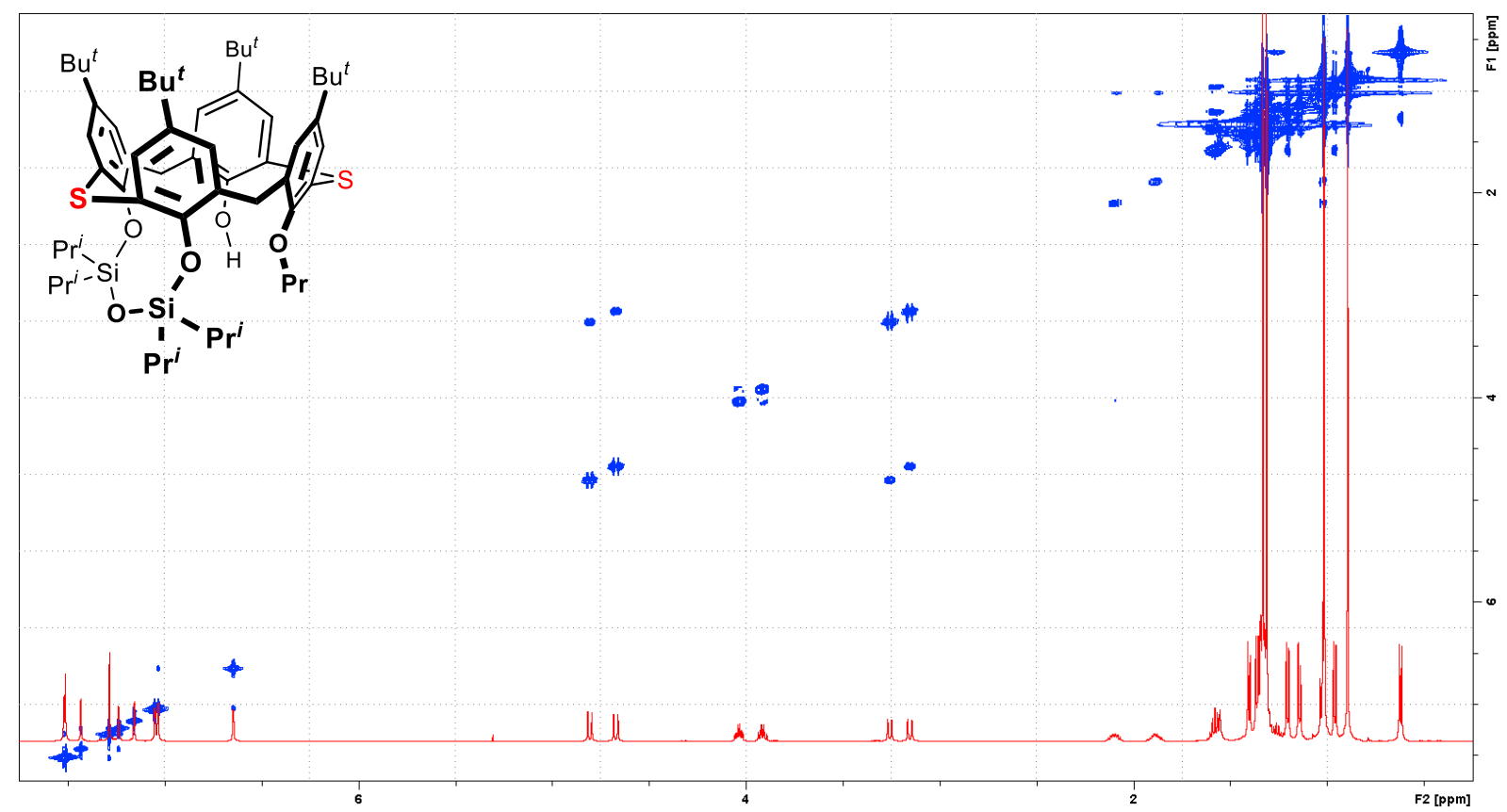

Figure S106. COSY NMR of compound $10\left(\mathrm{CDCl}_{3}\right)$.

14_Kortus_ESIpos_DK133_F2_1 4/3/2020 2:58:28 PM
MetOH

14 Kortus ESIpos DK133 F2 1 \#30-36 RT: 0.43-0.51 AV: 7 NL: 8.86E6

T: FTMS + c ESI Füll ms [250.00-2000.00]

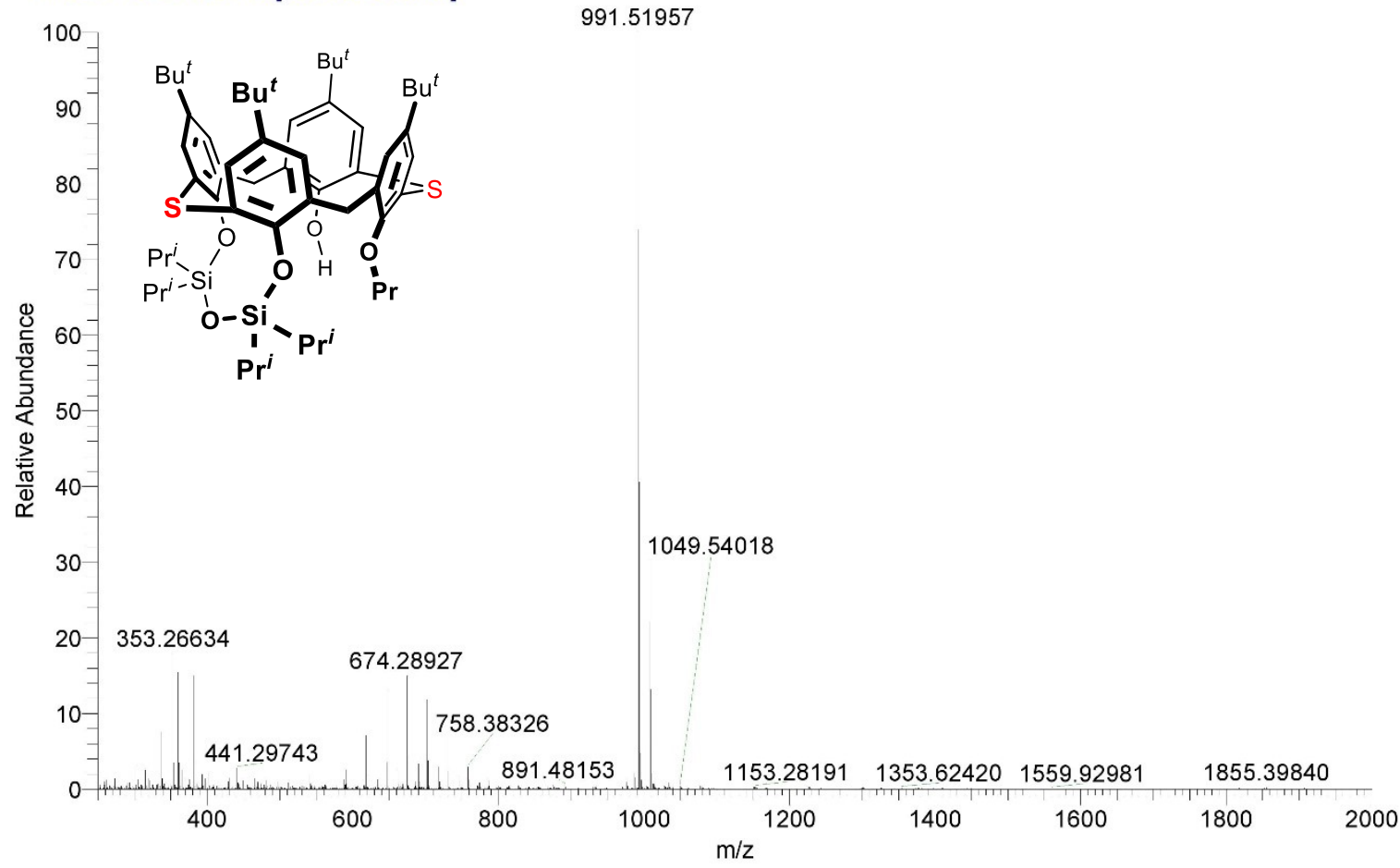

Figure S107. HRMS of compound $10\left(\mathrm{ESI}^{+}\right)$. 


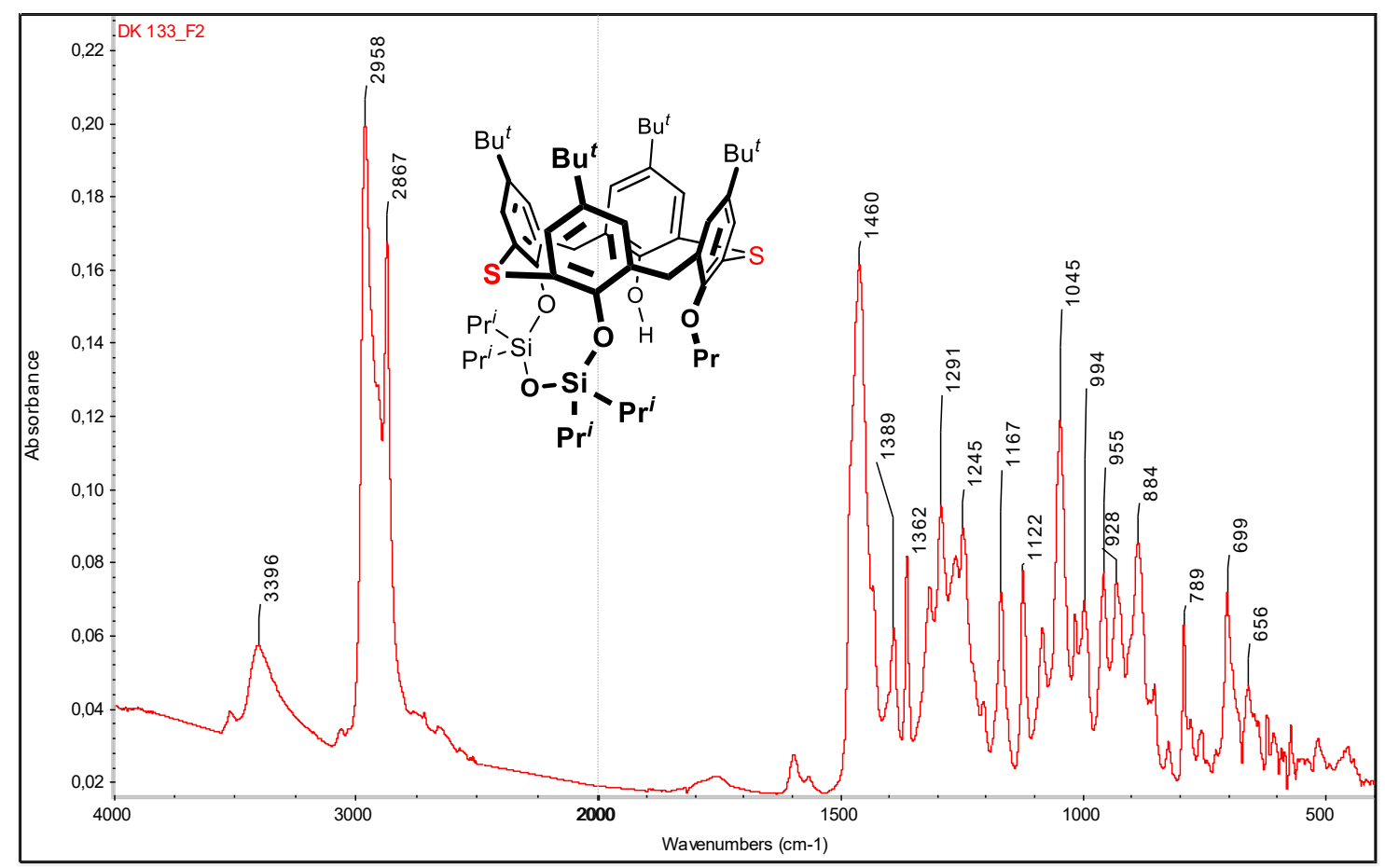

Figure S108. IR of compound 10 (ATR). 


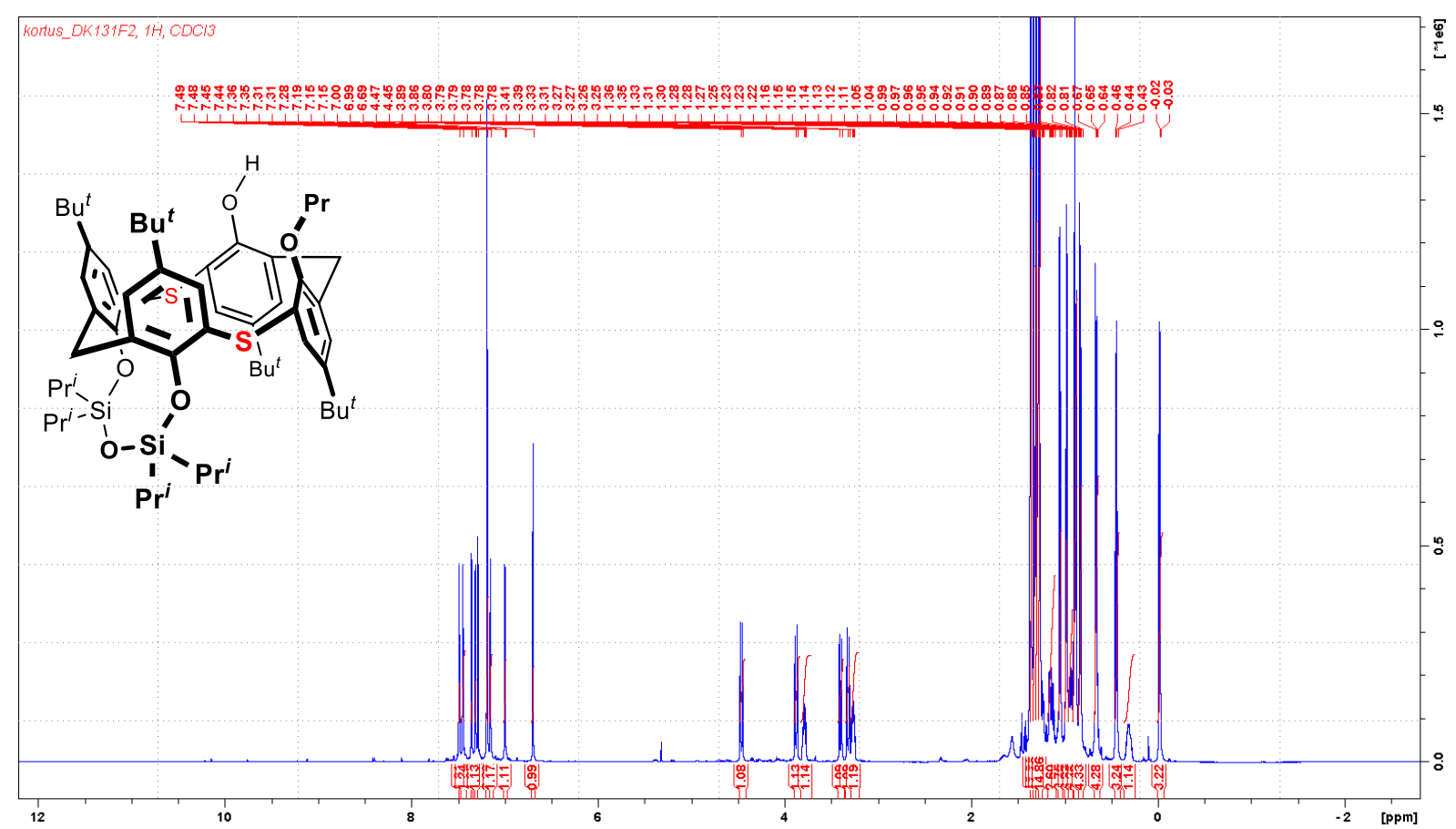

Figure S109. ${ }^{1} \mathrm{H}$ NMR of compound $11\left(\mathrm{CDCl}_{3}, 600 \mathrm{MHz}\right)$.

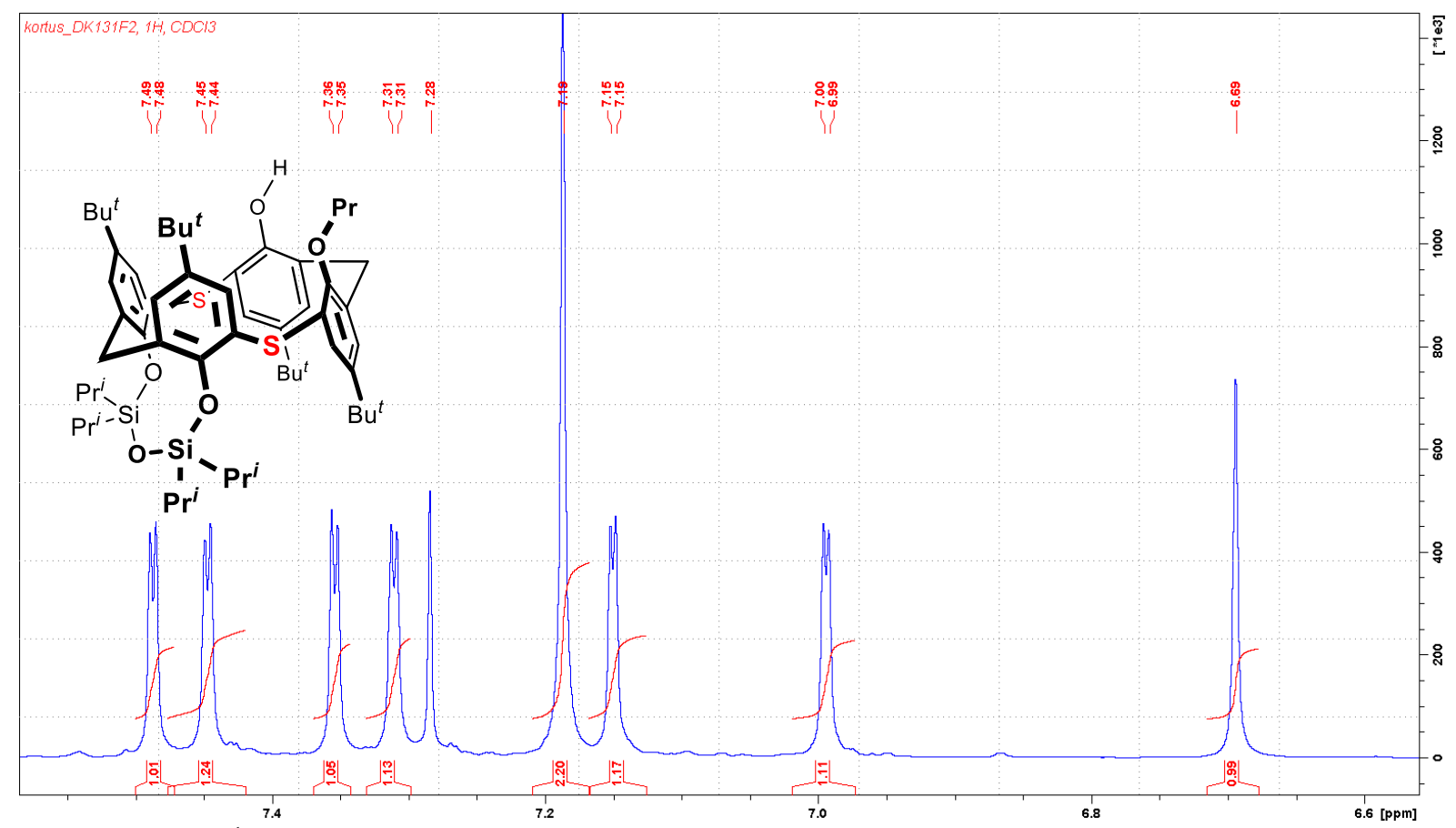

Figure S110. ${ }^{1} \mathrm{H}$ NMR of compound 11, aromatic region $\left(\mathrm{CDCl}_{3}, 600 \mathrm{MHz}\right)$. 


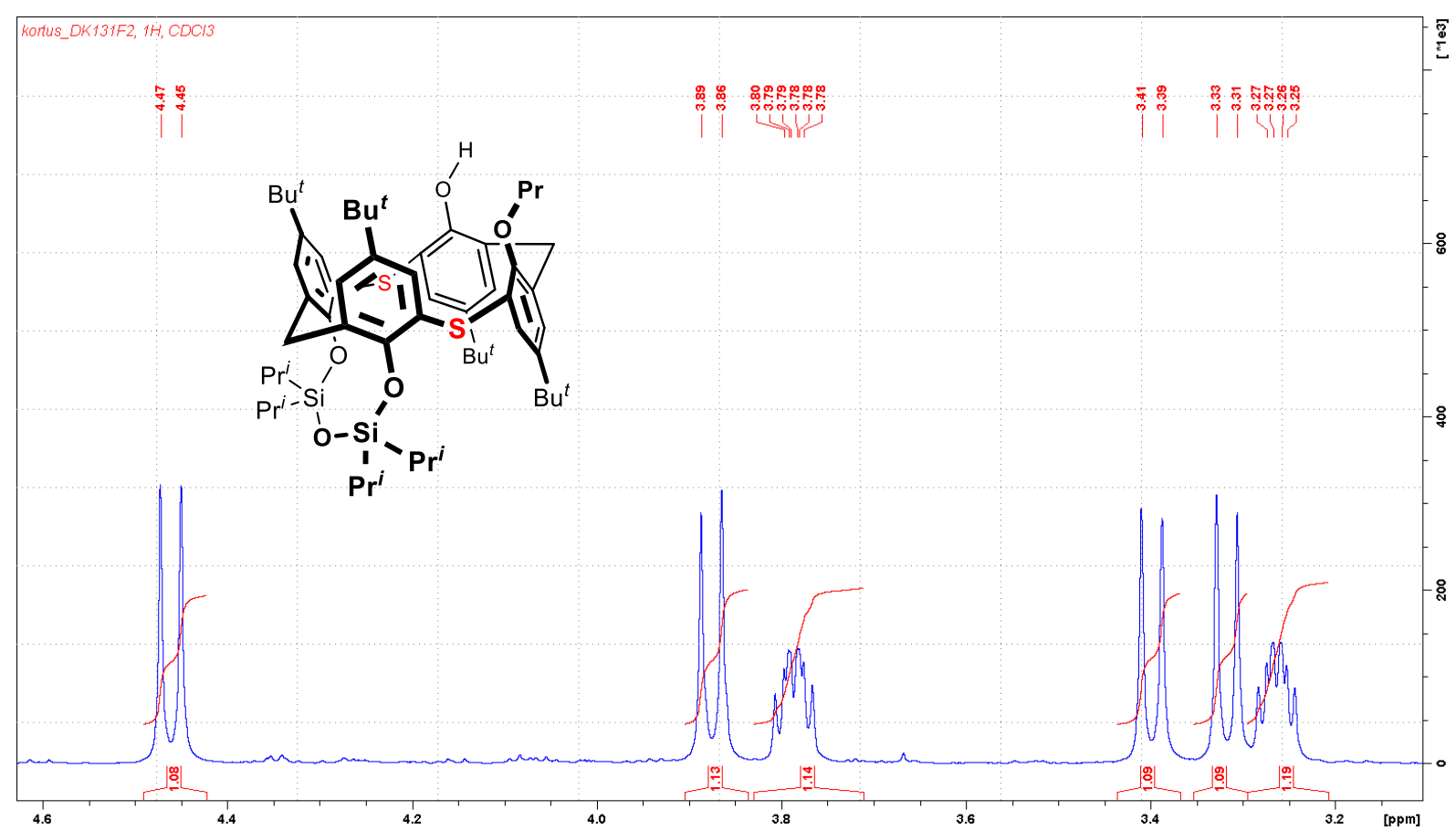

Figure S111. ${ }^{1} \mathrm{H}$ NMR of compound 11, bridge region $\left(\mathrm{CDCl}_{3}, 600 \mathrm{MHz}\right)$.

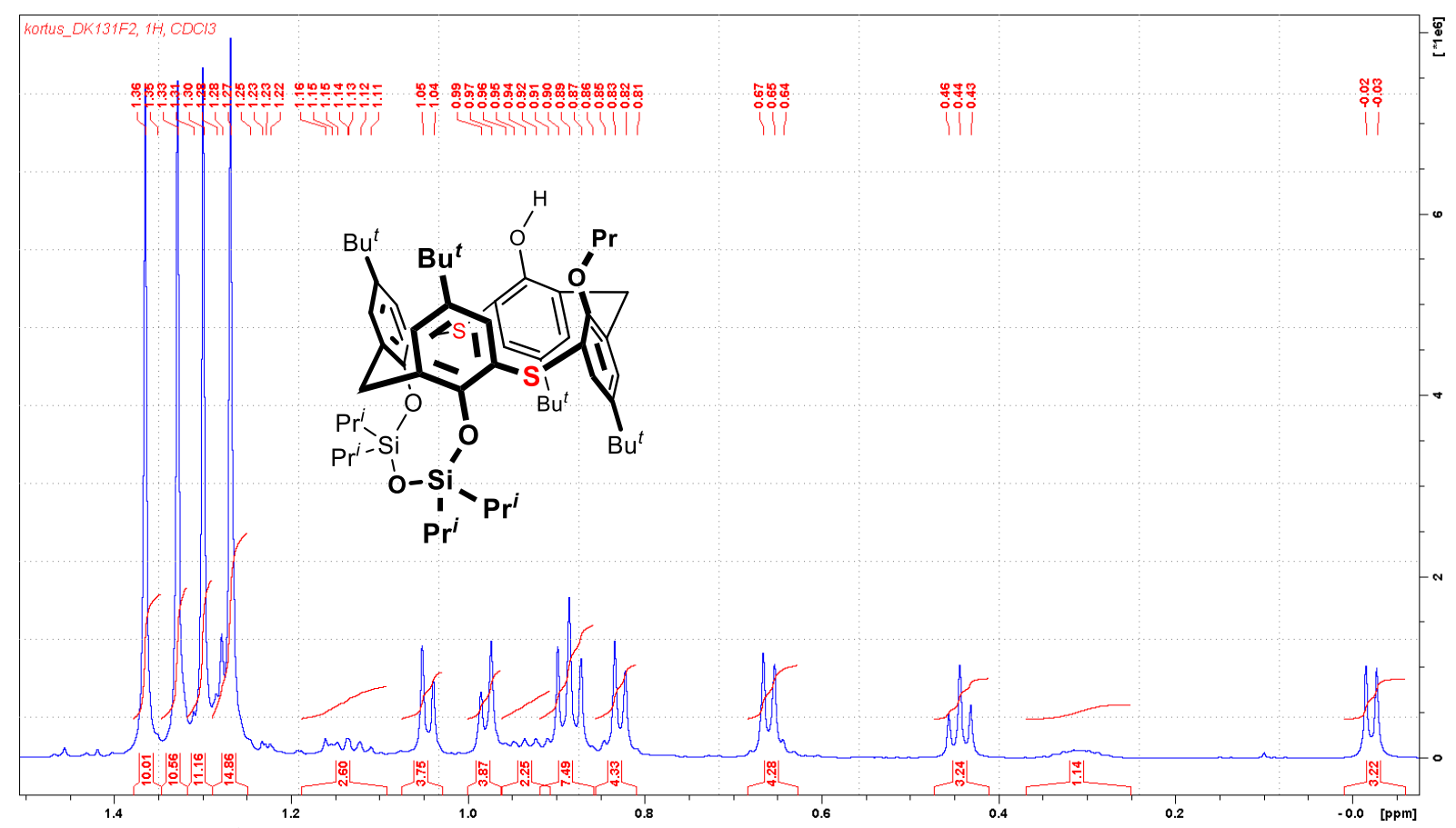

Figure S112. ${ }^{1} \mathrm{H}$ NMR of compound 11, aliphatic region $\left(\mathrm{CDCl}_{3}, 600 \mathrm{MHz}\right)$. 


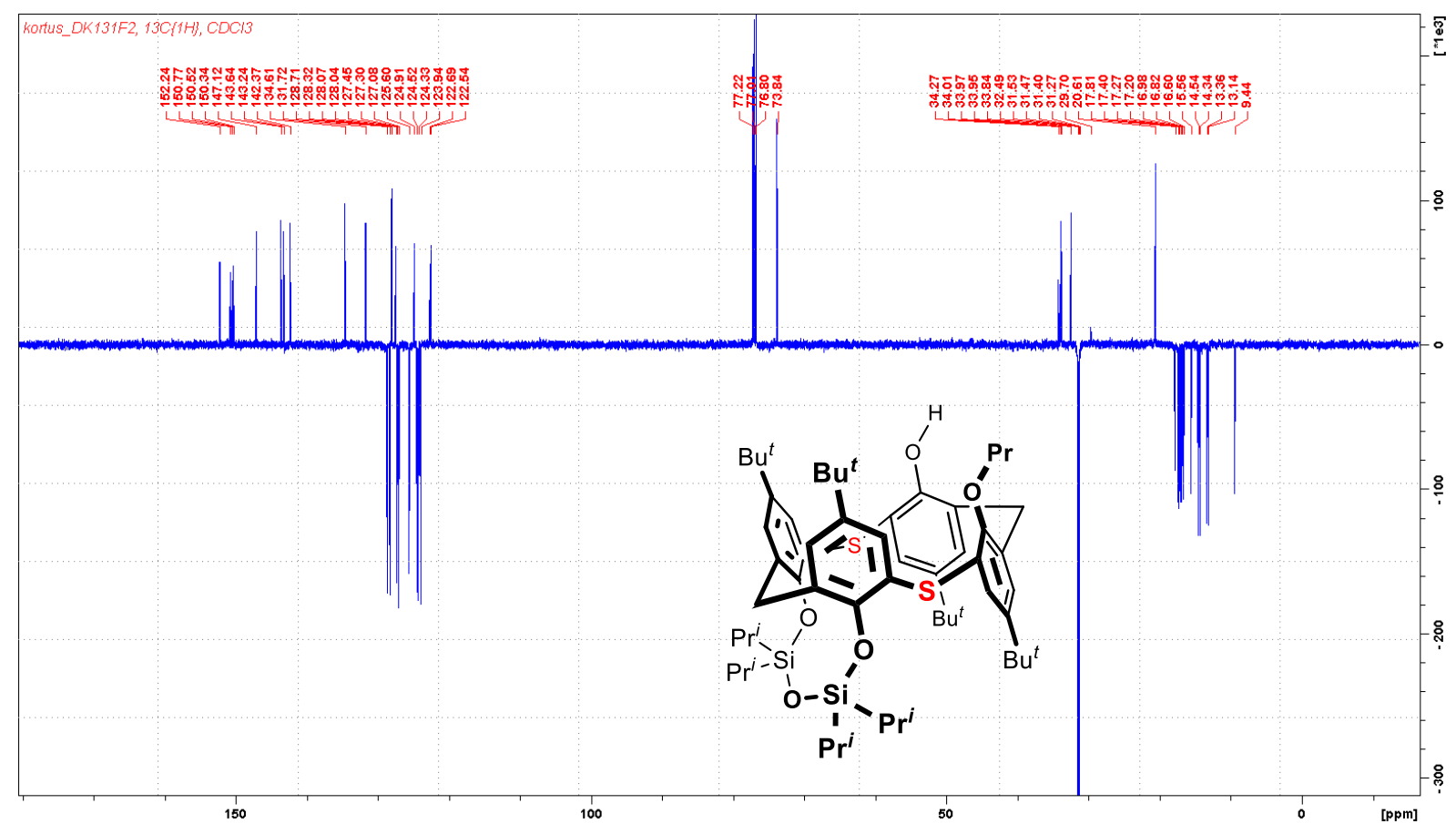

Figure S113. ${ }^{13} \mathrm{C}\left\{{ }^{1} \mathrm{H}\right\}$ NMR of compound $11\left(\mathrm{CDCl}_{3}, 151 \mathrm{MHz}\right)$.

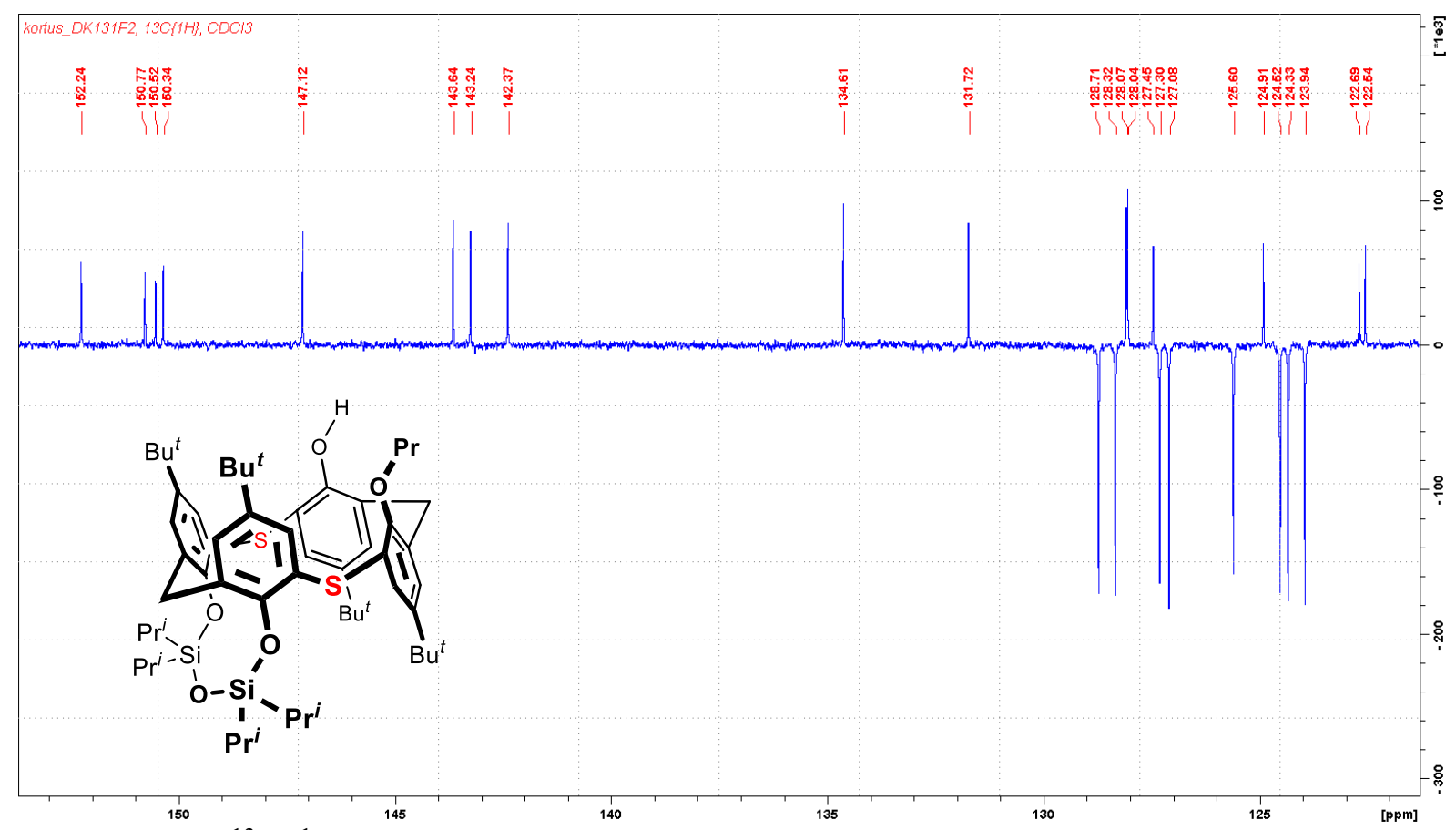

Figure S114. ${ }^{13} \mathrm{C}\left\{{ }^{1} \mathrm{H}\right\}$ NMR of compound 11, aromatic region $\left(\mathrm{CDCl}_{3}, 151 \mathrm{MHz}\right)$. 


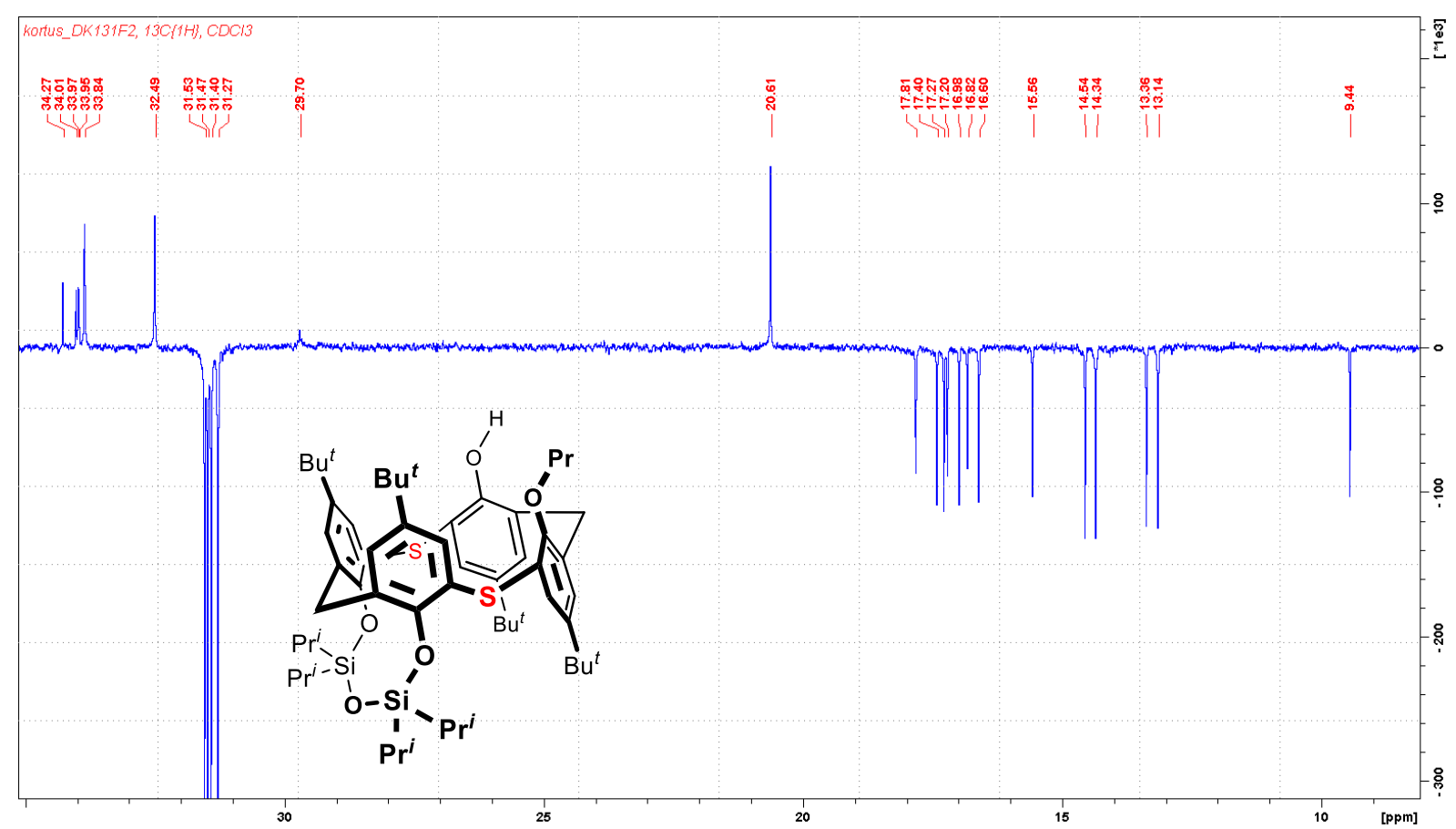

Figure S115. ${ }^{13} \mathrm{C}\left\{{ }^{1} \mathrm{H}\right\}$ NMR of compound 11, aliphatic region $\left(\mathrm{CDCl}_{3}, 151 \mathrm{MHz}\right)$.

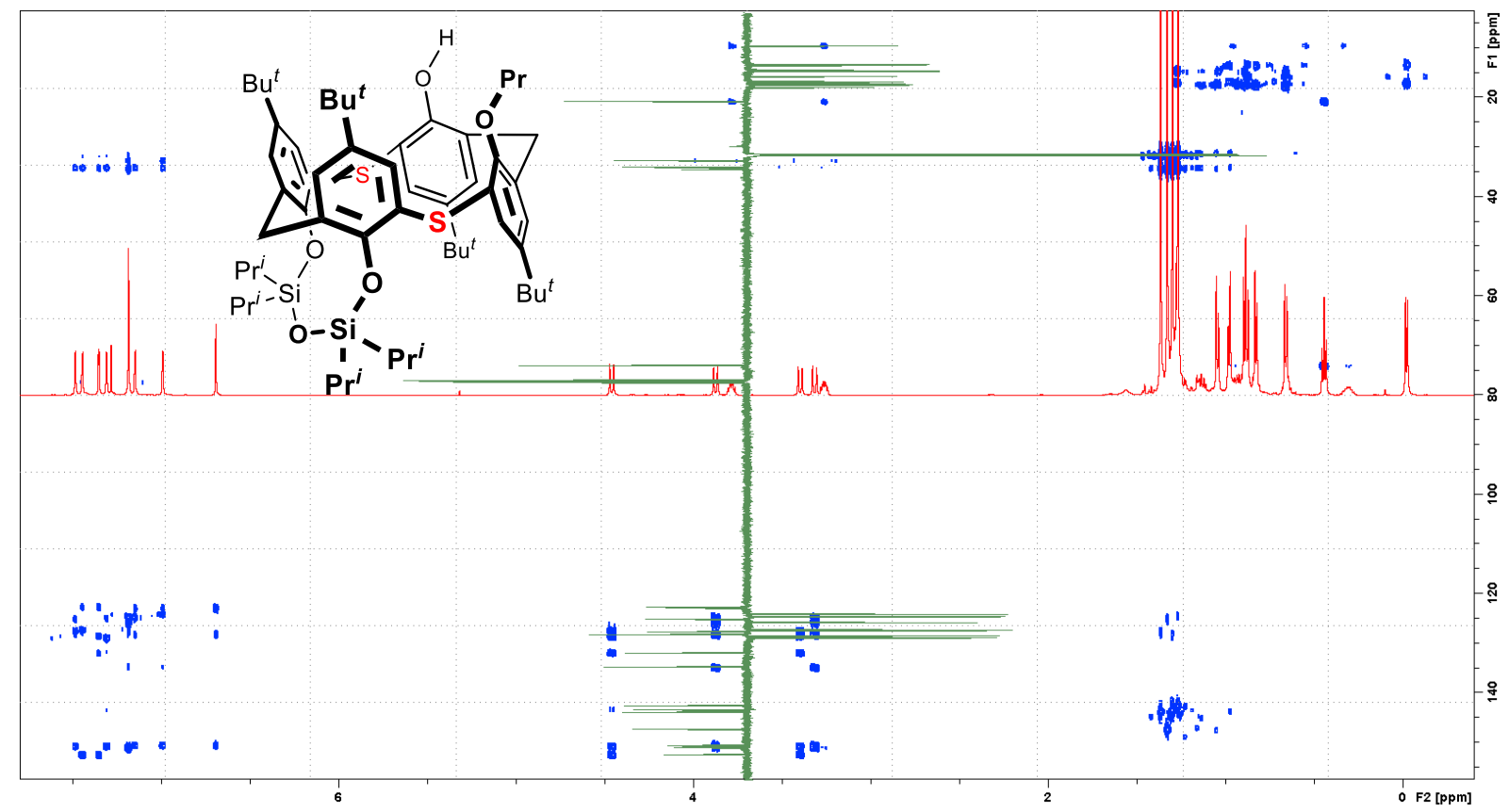

Figure S116. HMBC NMR of compound $11\left(\mathrm{CDCl}_{3}\right)$. 


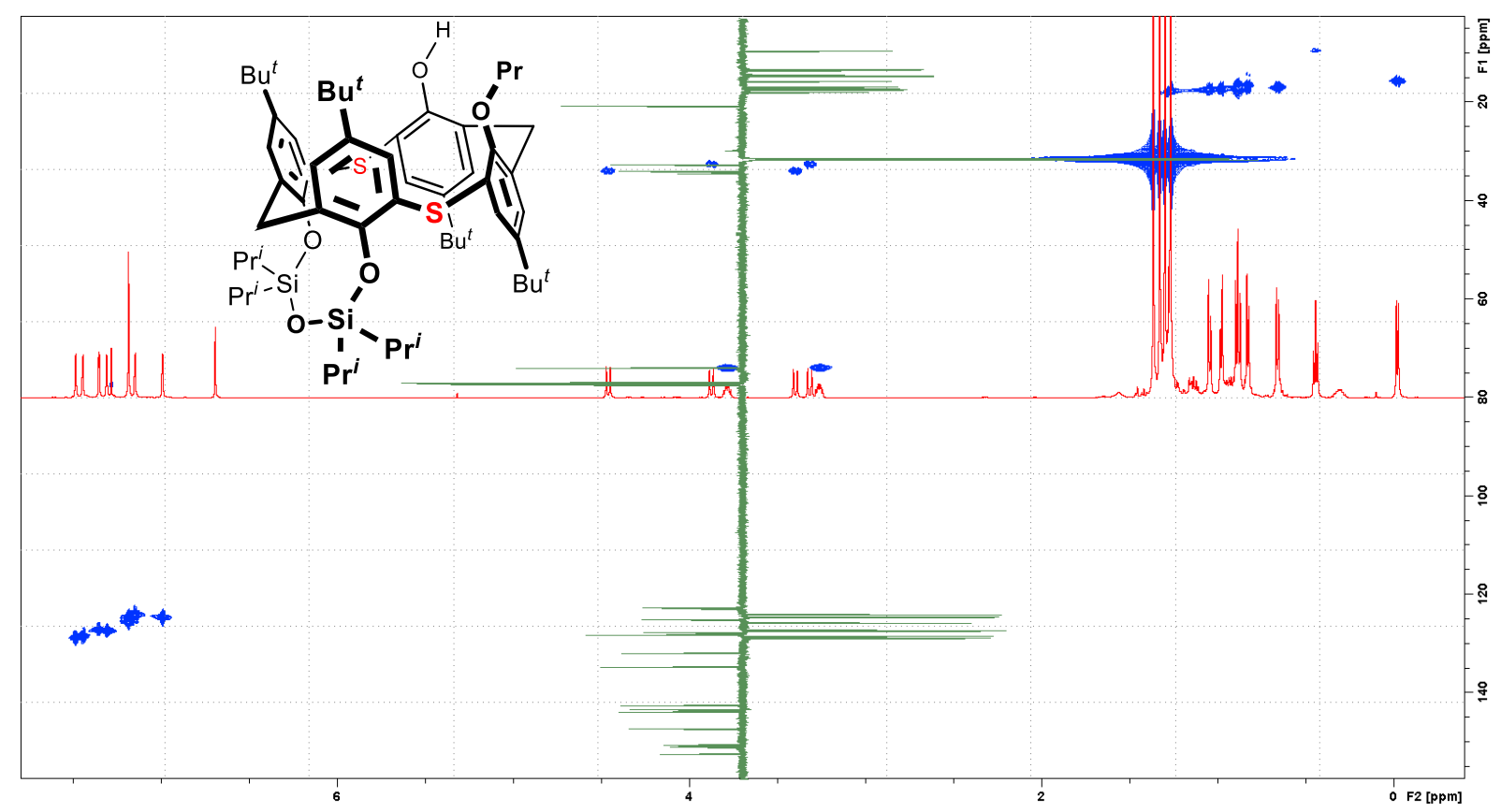

Figure S117. HMQC NMR of compound $11\left(\mathrm{CDCl}_{3}\right)$.

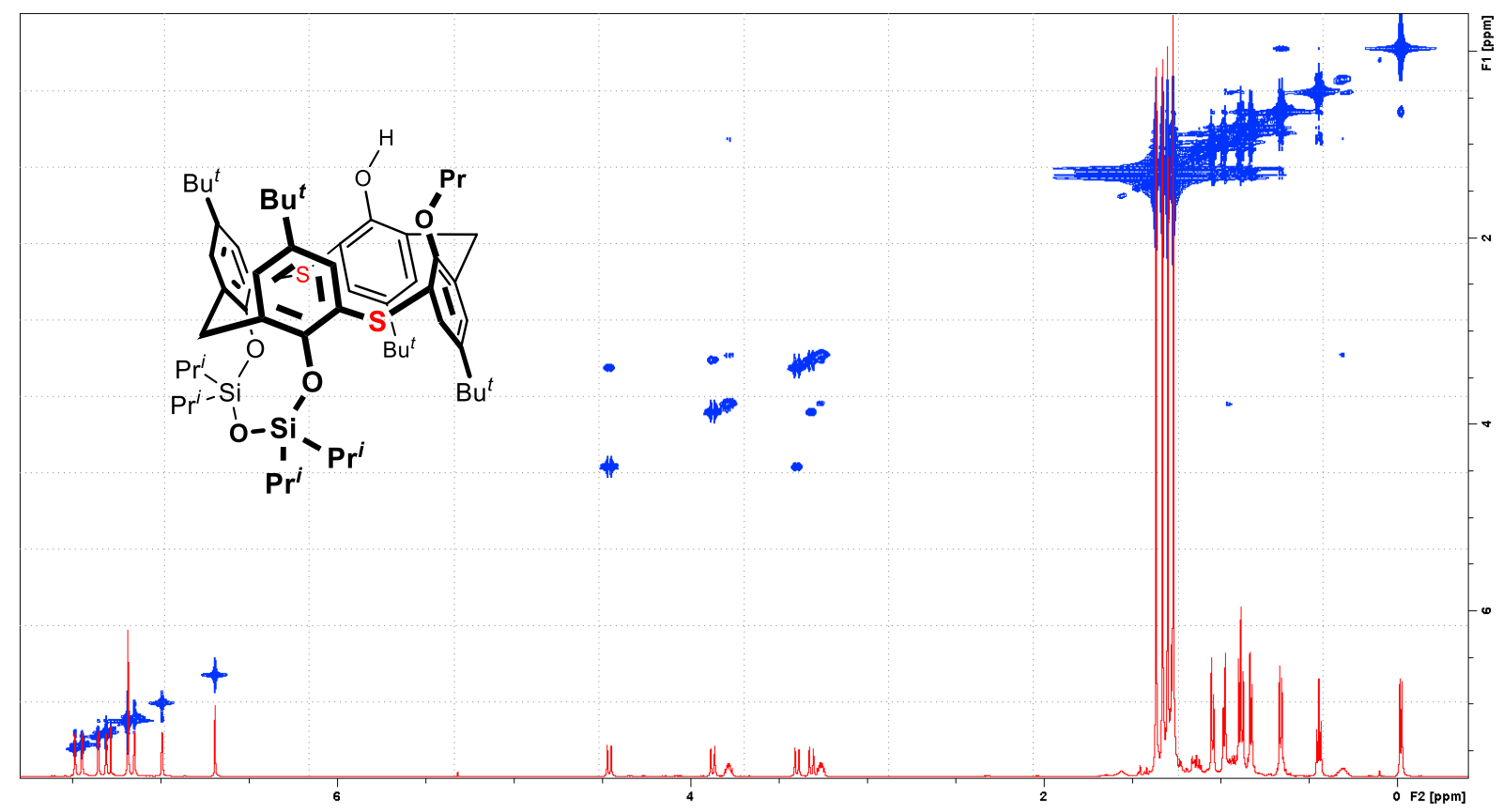

Figure S118. COSY NMR of compound $11\left(\mathrm{CDCl}_{3}\right)$. 


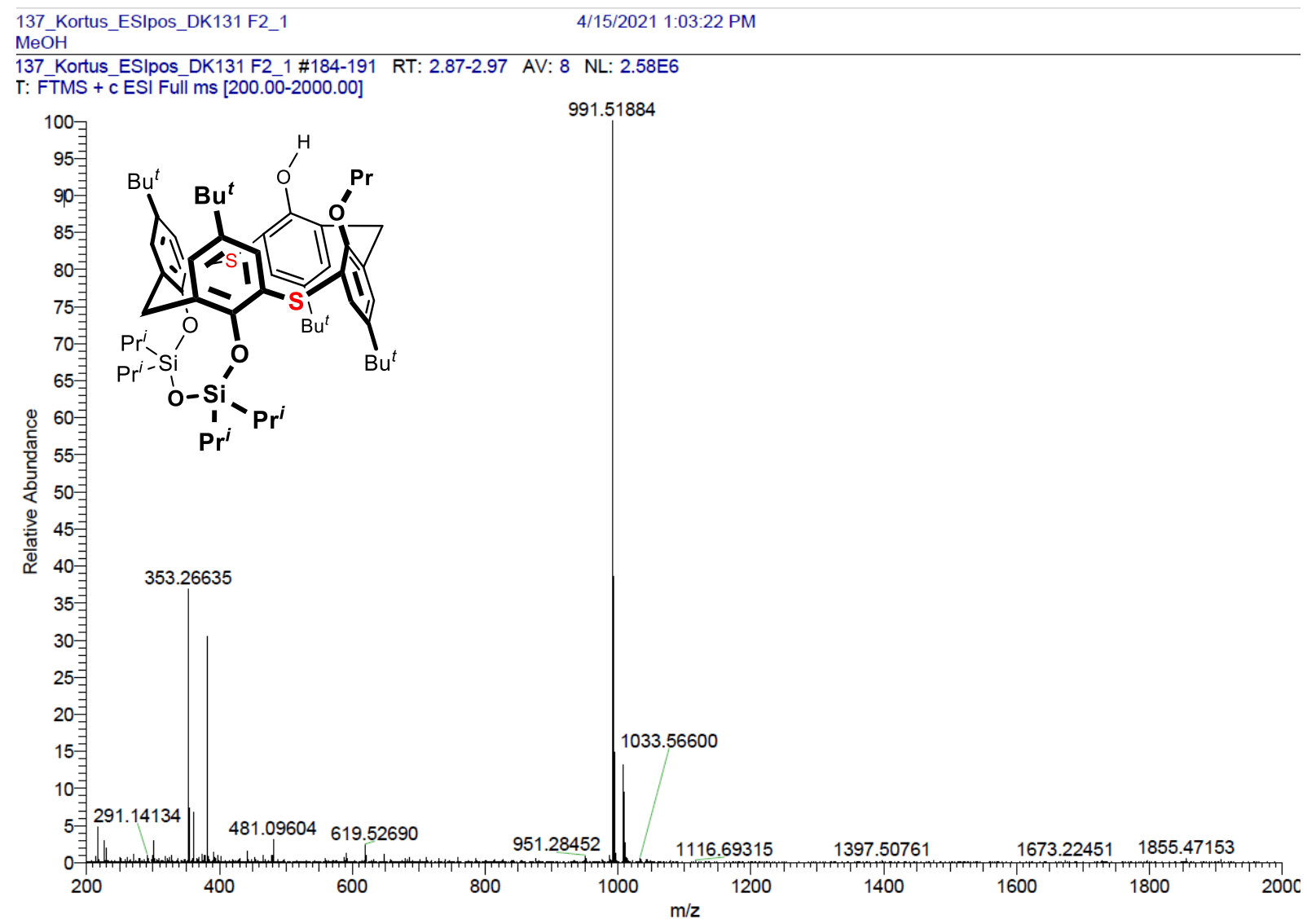

Figure S119. HRMS of compound $11\left(\mathrm{ESI}^{+}\right)$.

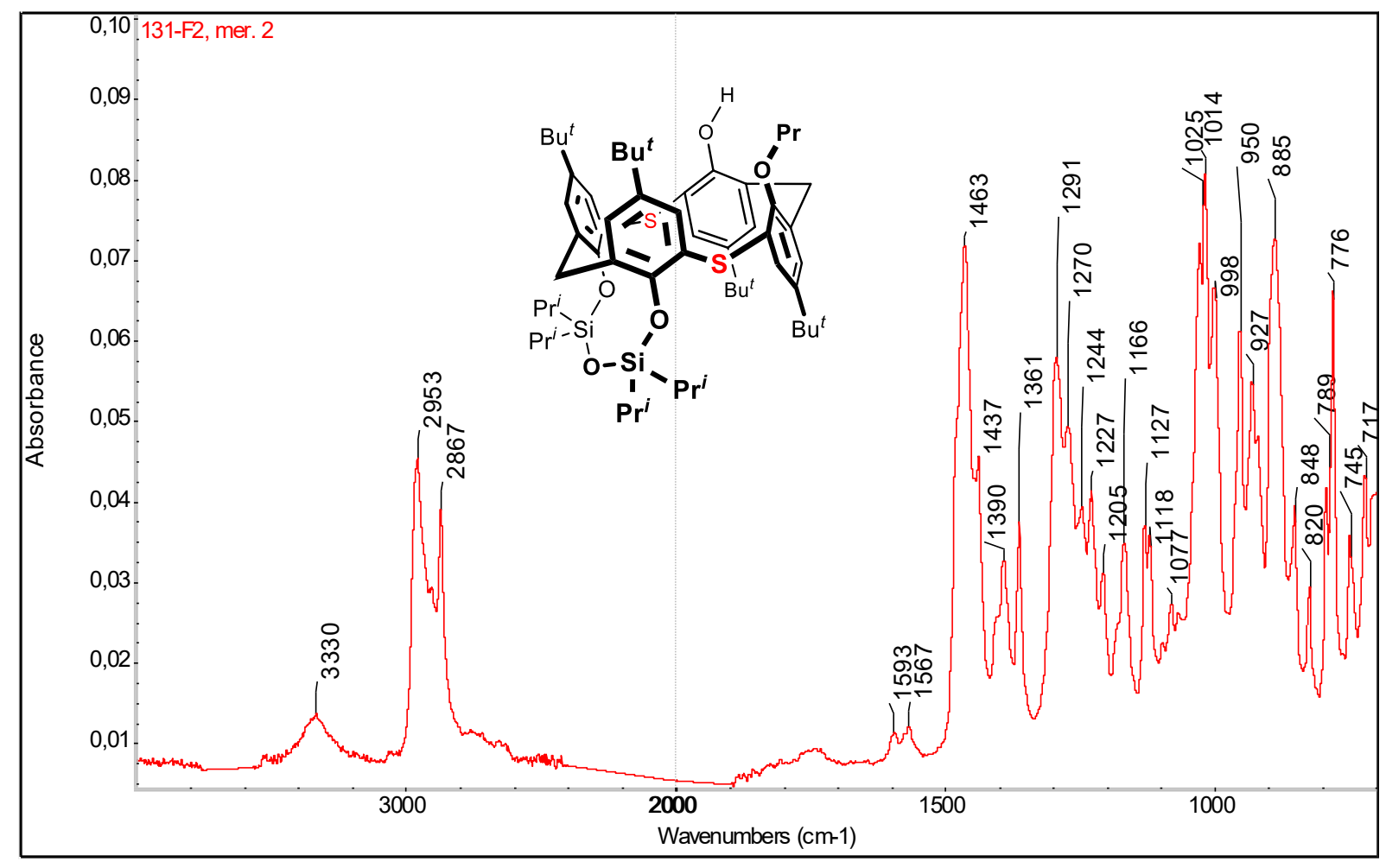

Figure S120. IR of compound 11 (ATR). 


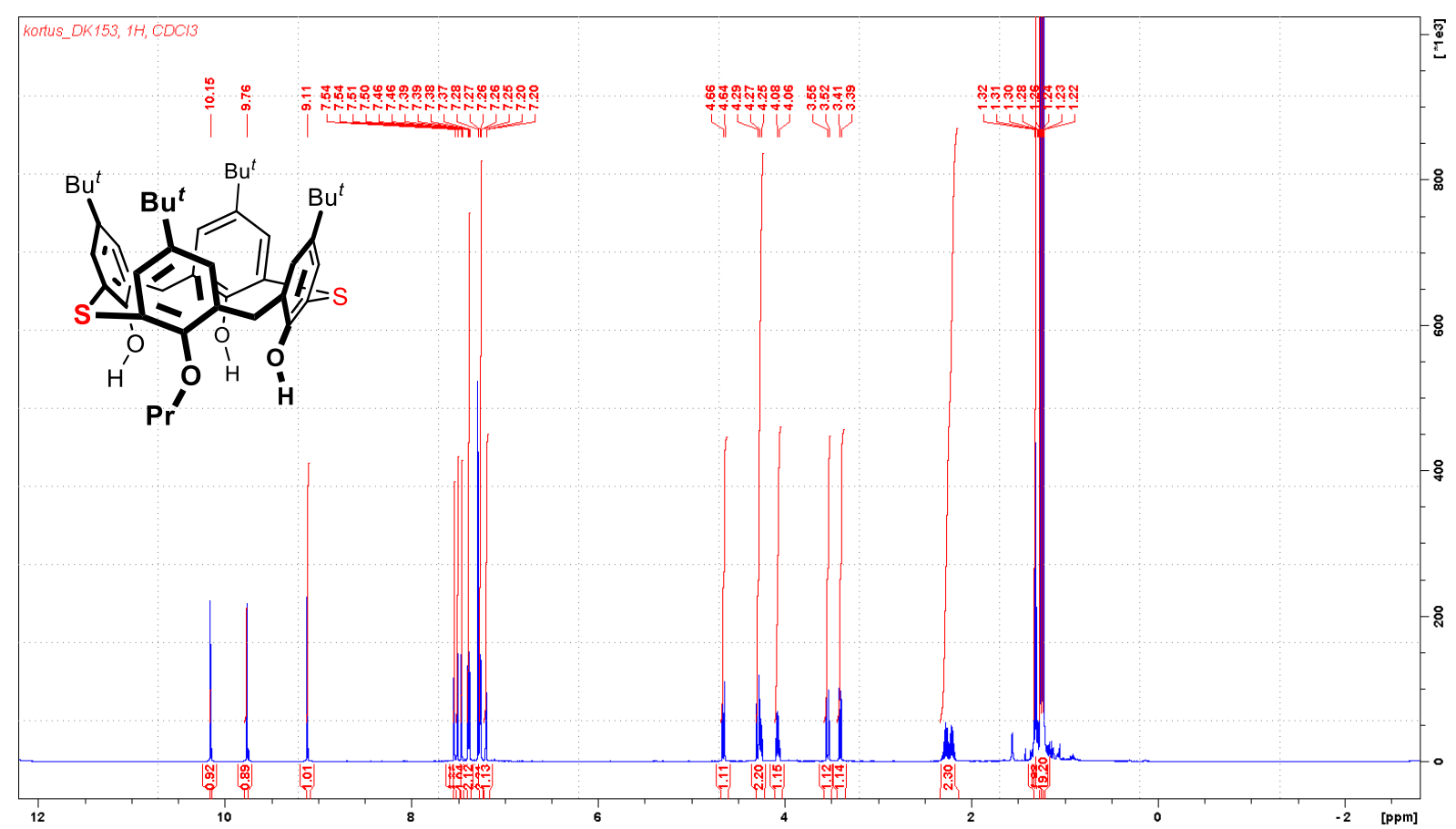

Figure S121. ${ }^{1} \mathrm{H}$ NMR of compound $13\left(\mathrm{CDCl}_{3}, 600 \mathrm{MHz}\right)$.

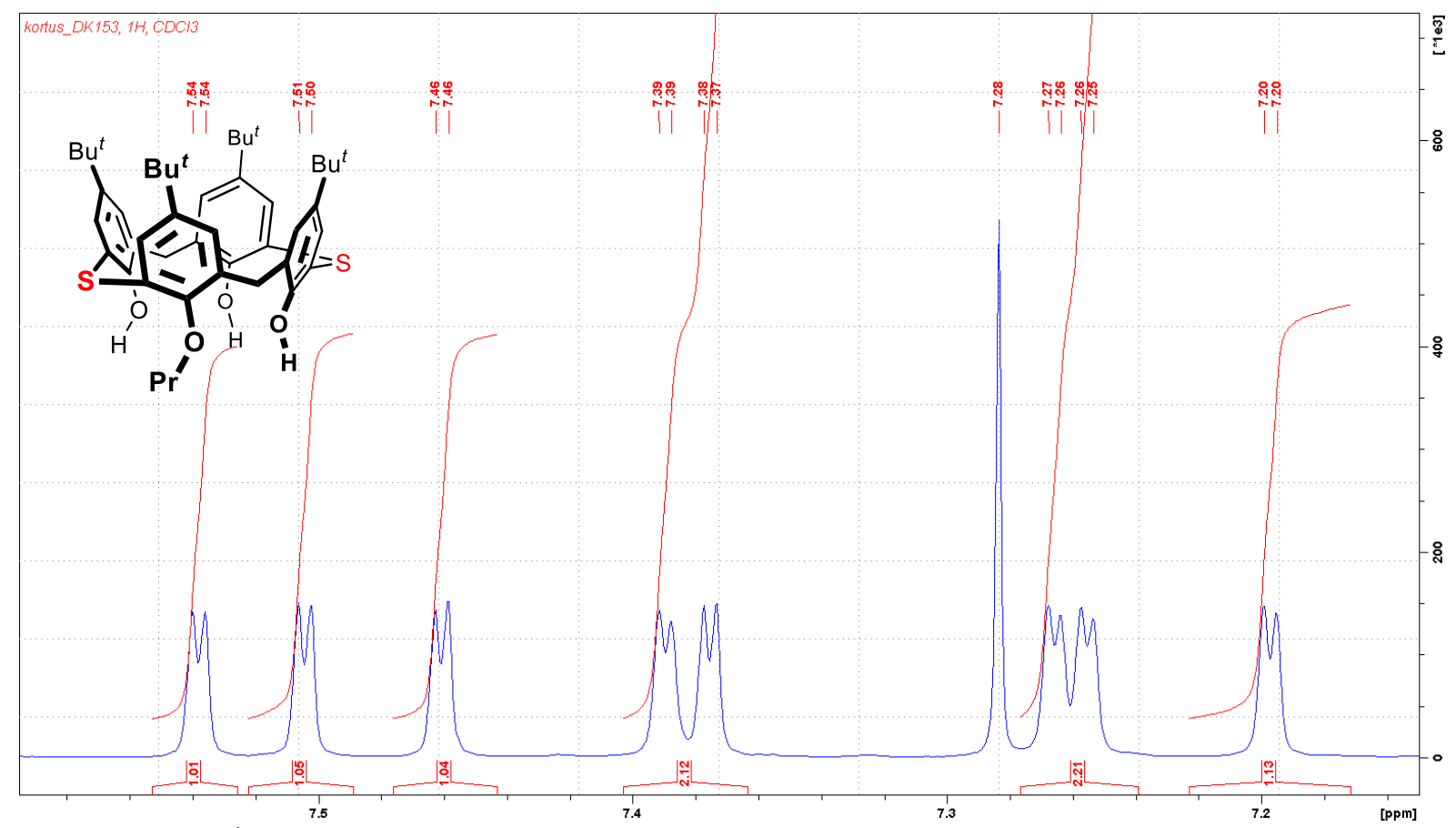

Figure S121. ${ }^{1} \mathrm{H}$ NMR of compound 13 , aromatic region $\left(\mathrm{CDCl}_{3}, 600 \mathrm{MHz}\right)$. 


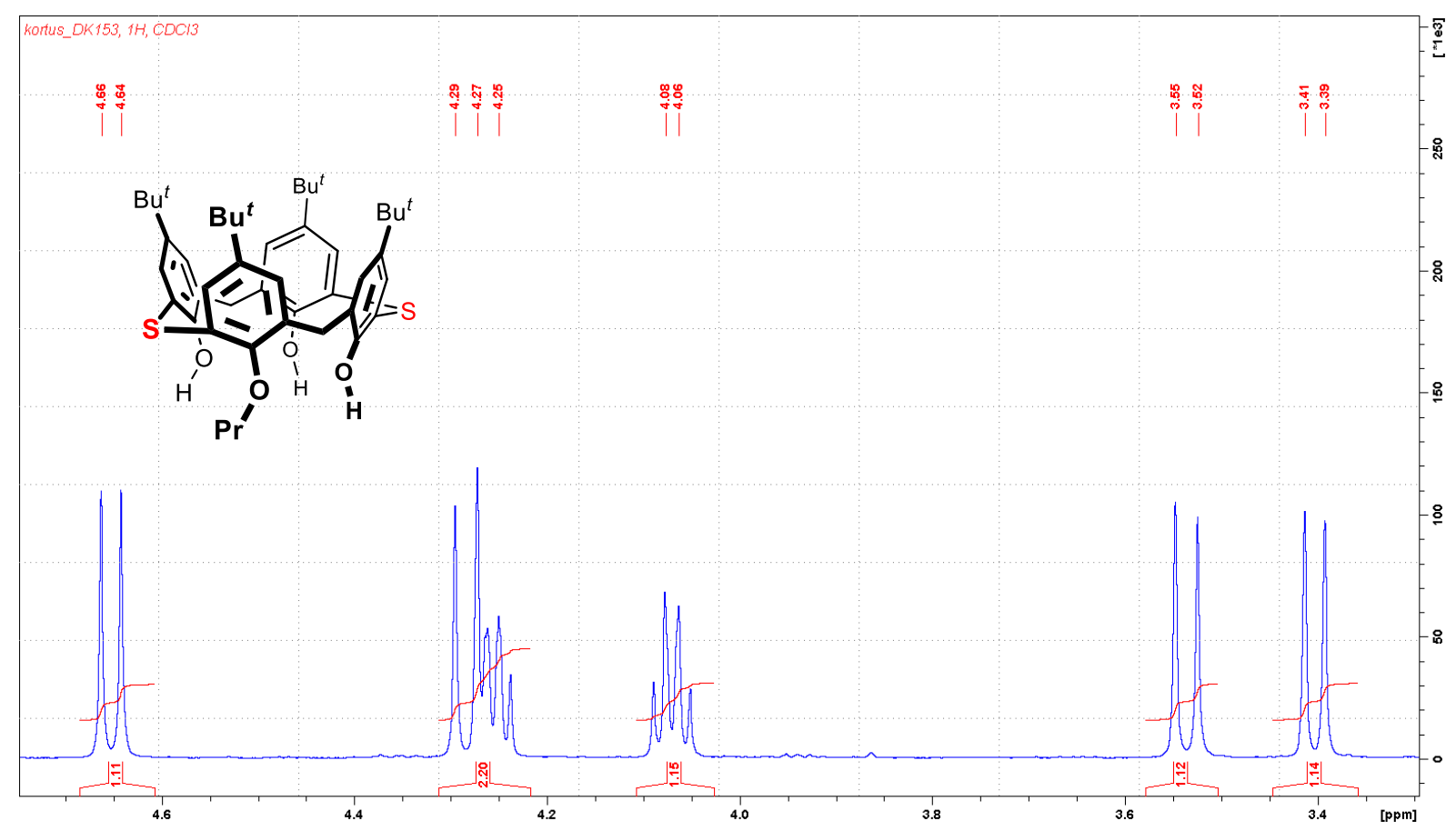

Figure S122. ${ }^{1} \mathrm{H}$ NMR of compound 13 , bridge region $\left(\mathrm{CDCl}_{3}, 600 \mathrm{MHz}\right)$.

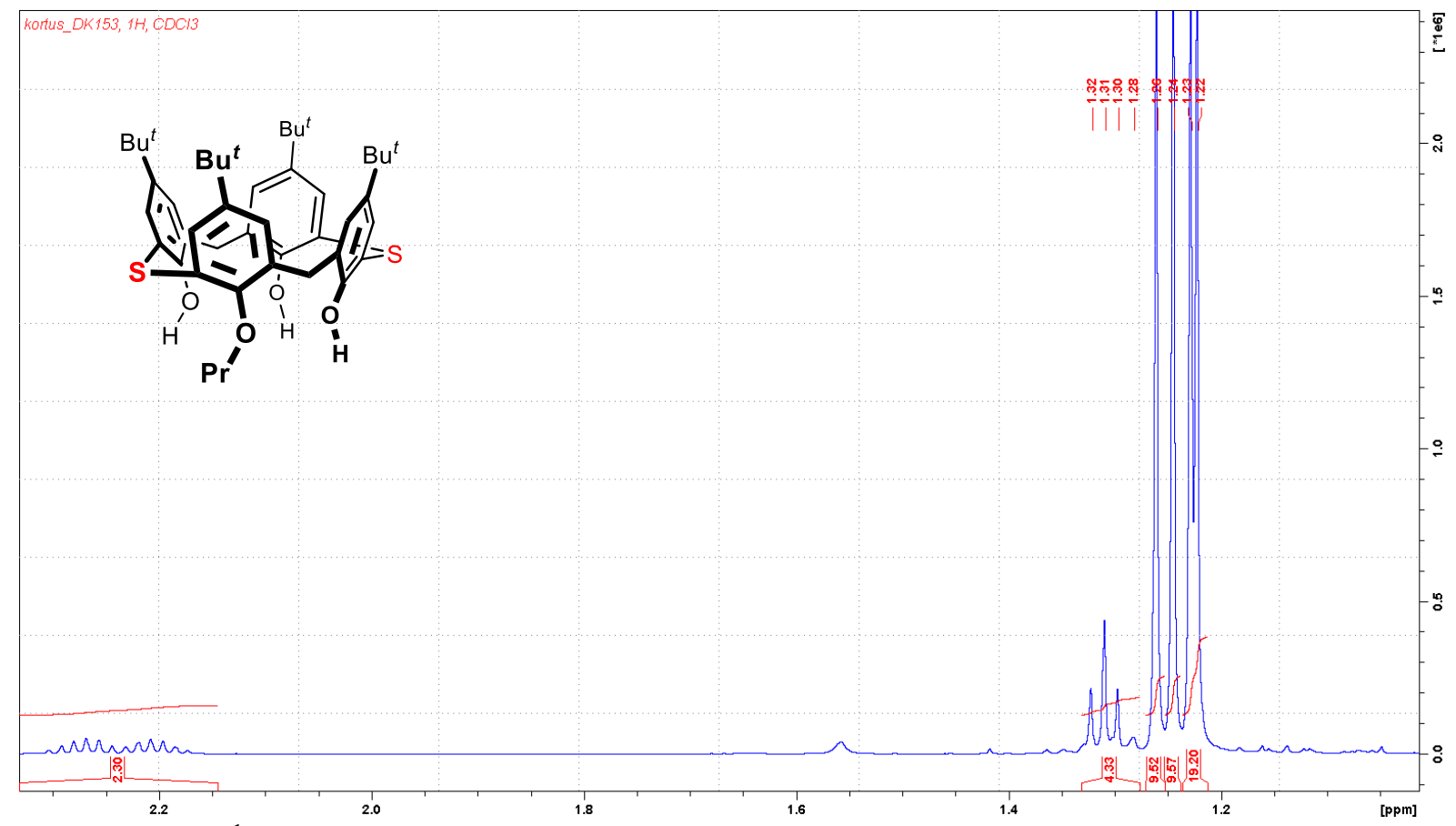

Figure S123. ${ }^{1} \mathrm{H}$ NMR of compound 13 , aliphatic region $\left(\mathrm{CDCl}_{3}, 600 \mathrm{MHz}\right)$. 


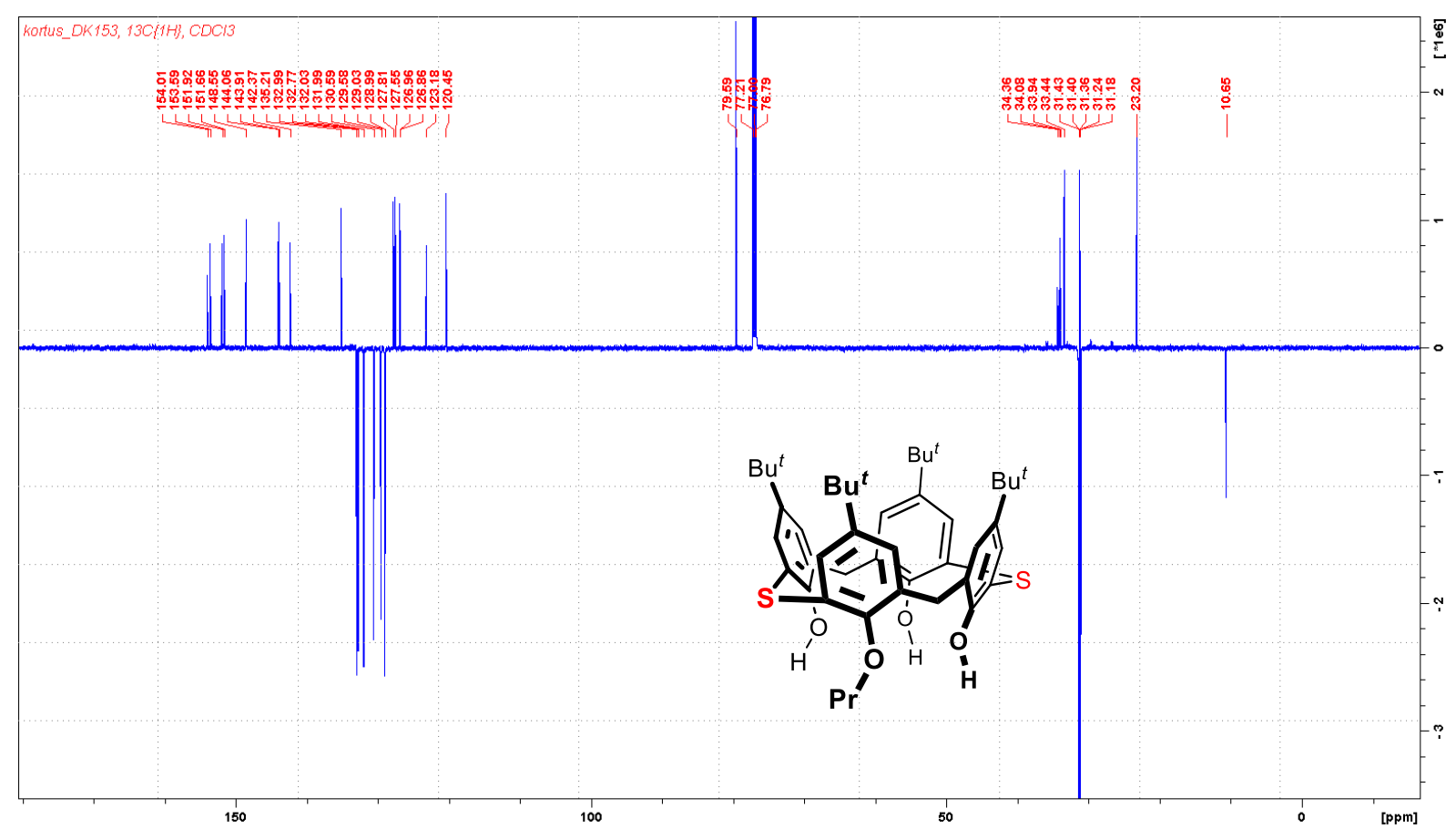

Figure S124. ${ }^{13} \mathrm{C}\left\{{ }^{1} \mathrm{H}\right\}$ NMR of compound $13\left(\mathrm{CDCl}_{3}, 151 \mathrm{MHz}\right)$.

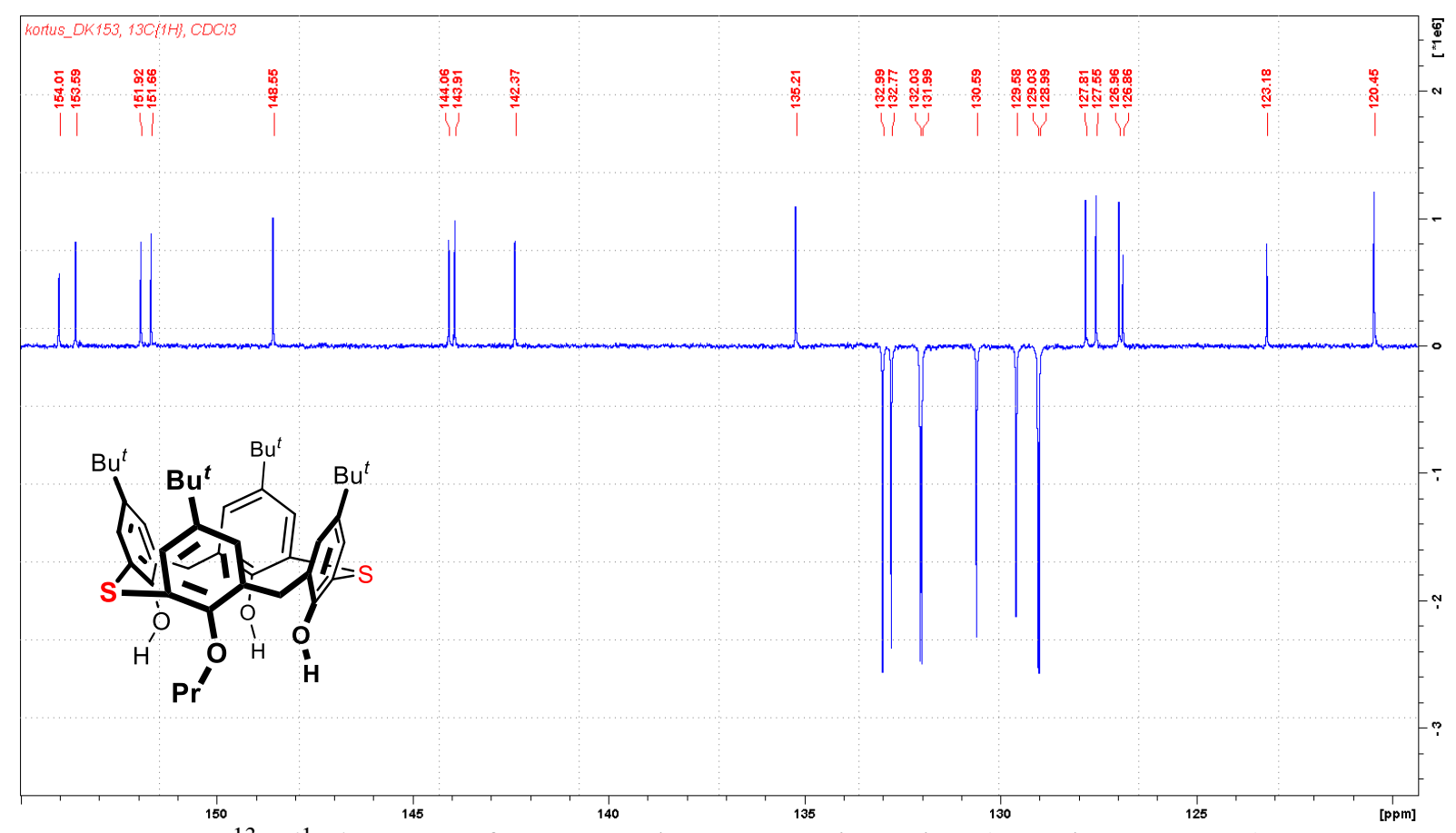

Figure S125. ${ }^{13} \mathrm{C}\left\{{ }^{1} \mathrm{H}\right\} \mathrm{NMR}$ of compound 13, aromatic region $\left(\mathrm{CDCl}_{3}, 151 \mathrm{MHz}\right)$. 


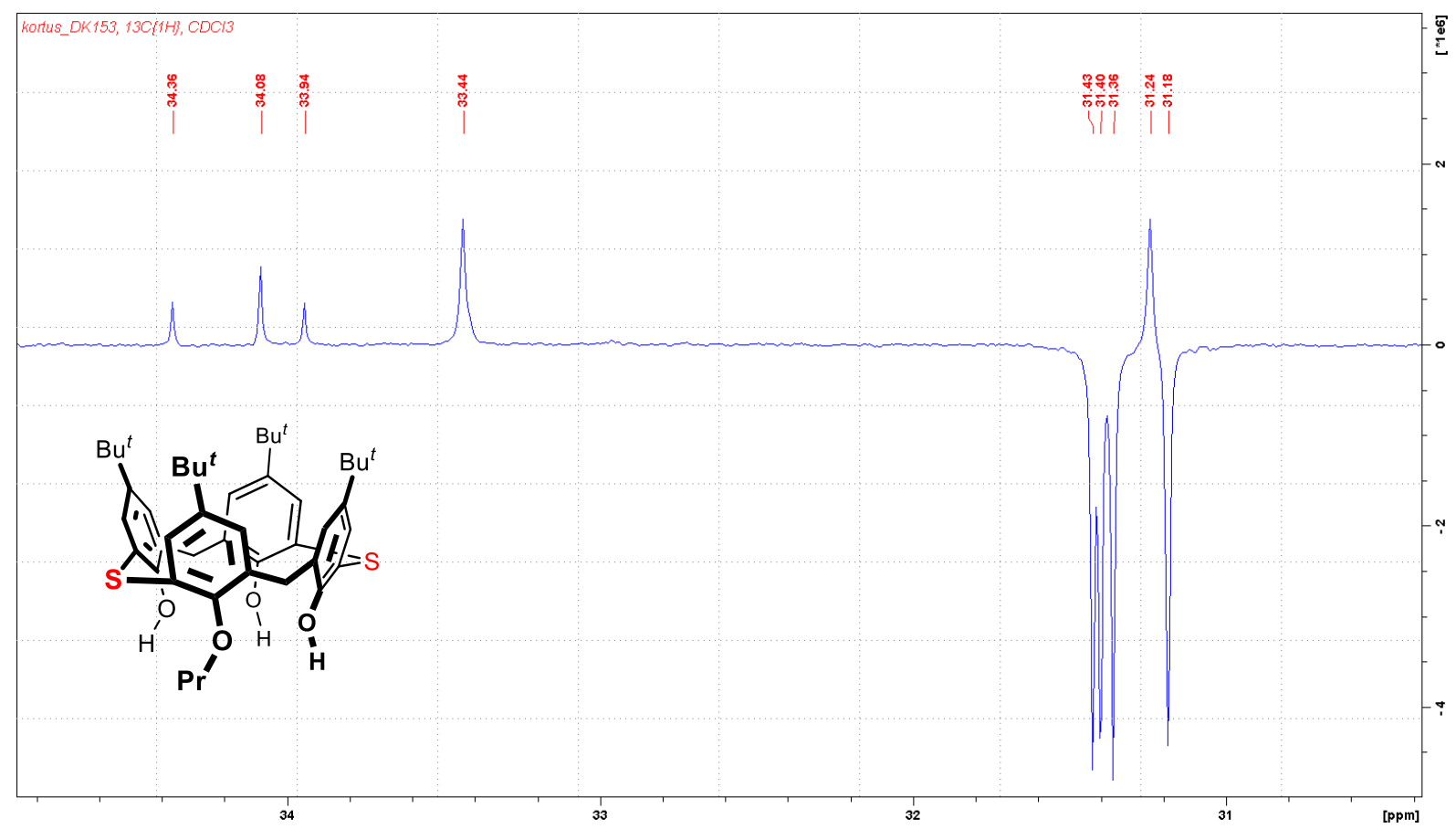

Figure S126. ${ }^{13} \mathrm{C}\left\{{ }^{1} \mathrm{H}\right\}$ NMR of compound 13, aliphatic region $\left(\mathrm{CDCl}_{3}, 151 \mathrm{MHz}\right)$.

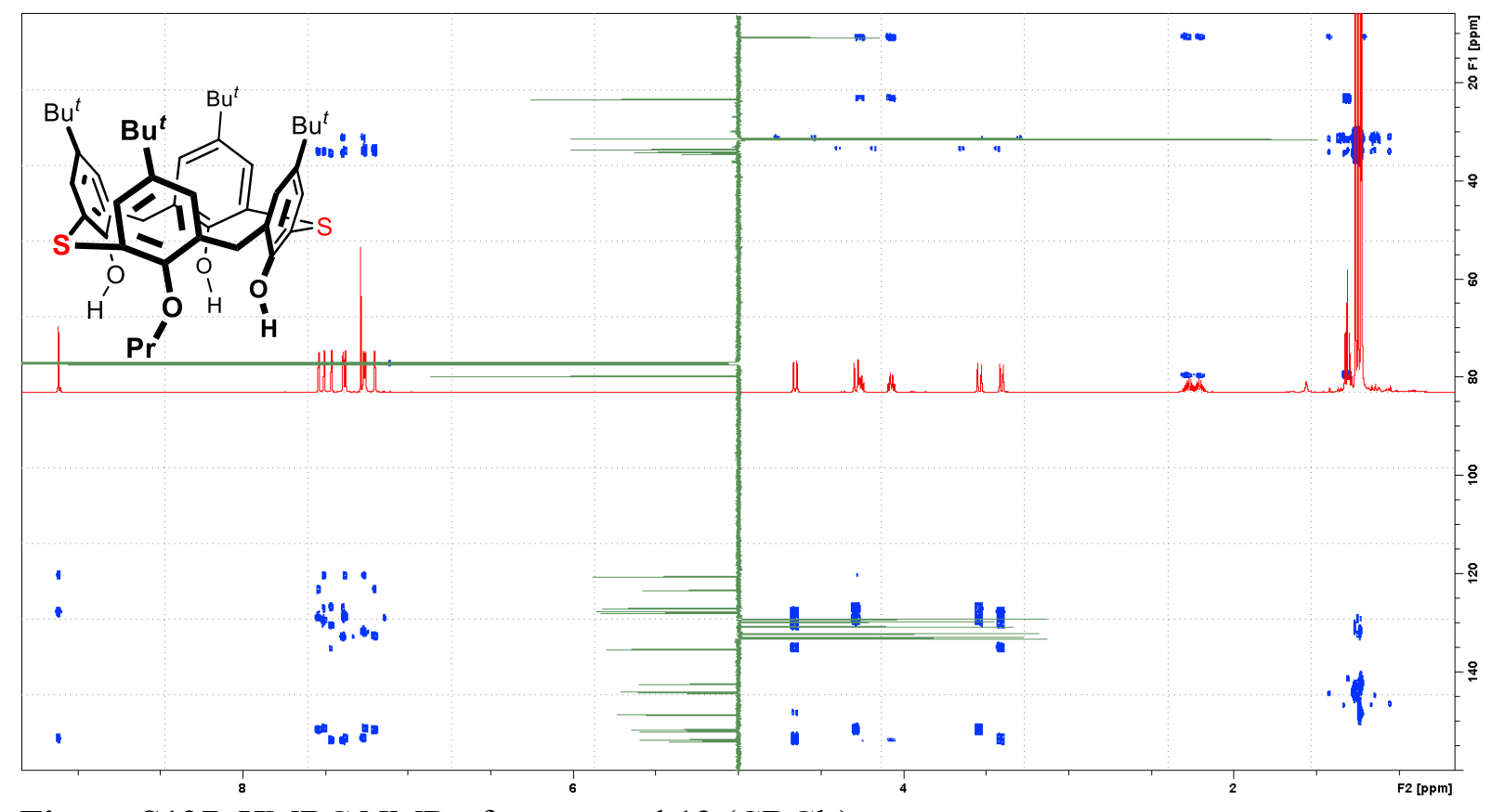

Figure S127. HMBC NMR of compound $13\left(\mathrm{CDCl}_{3}\right)$. 


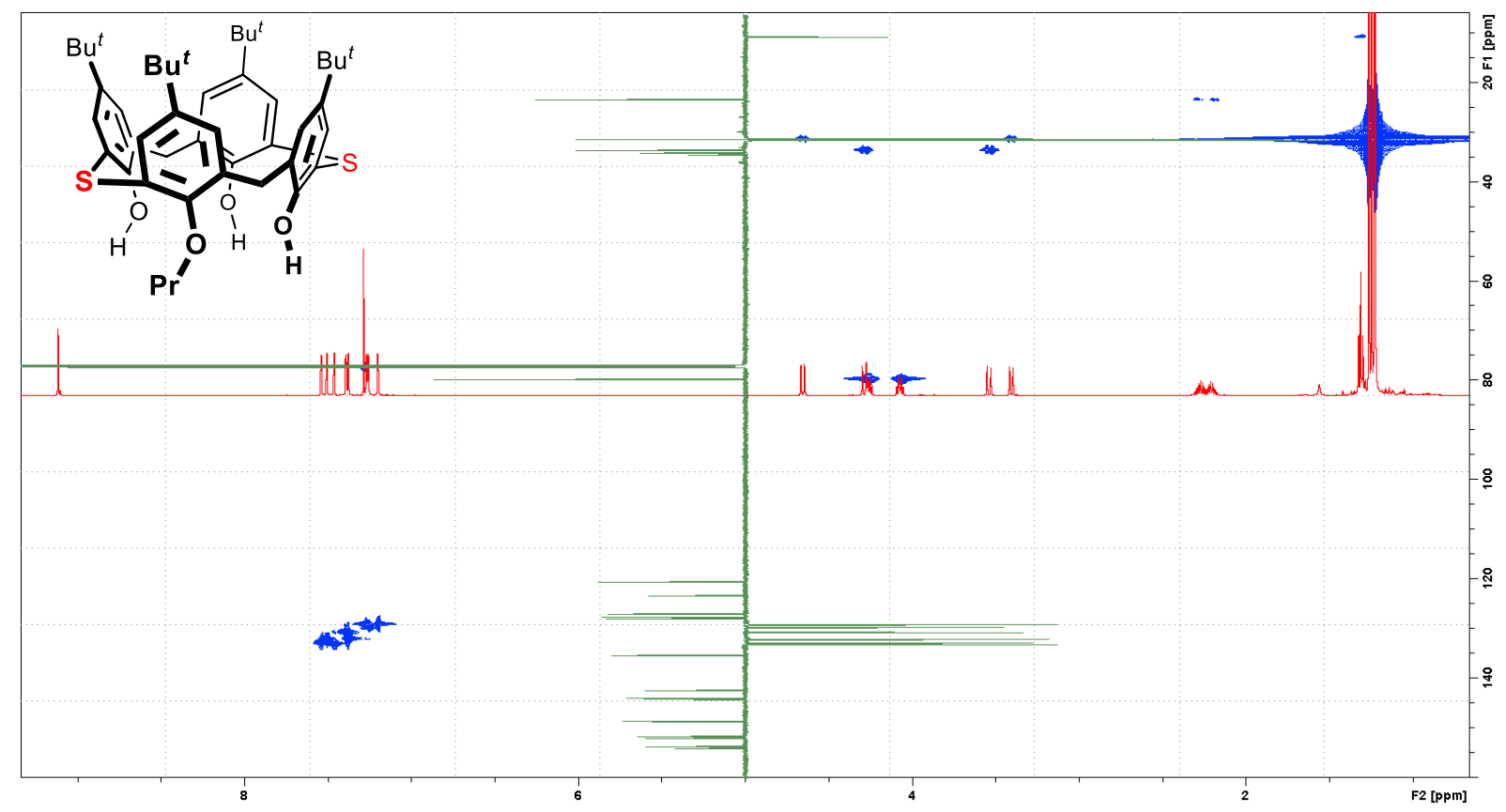

Figure S128. HMQC NMR of compound $13\left(\mathrm{CDCl}_{3}\right)$.

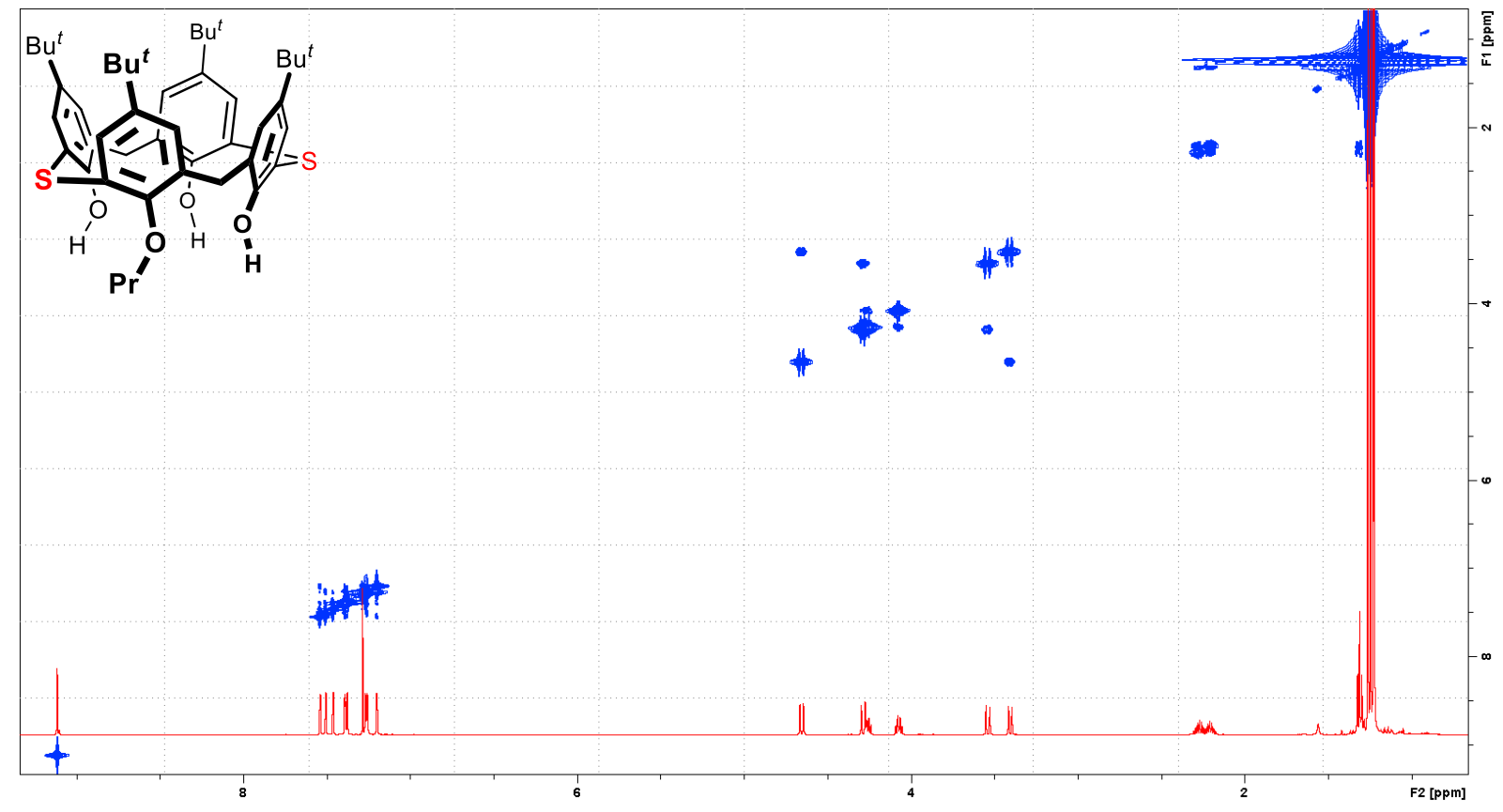

Figure S129. COSY NMR of compound $13\left(\mathrm{CDCl}_{3}\right)$. 
14_Kortus_ESIpos_DK153_1

4/3/2020 3.21:00 PM

14_Kortus_ESIpos_DK153_1 \#10-16 RT: 0.13-0.22 AV: 7 NL: $1.10 E 7$

T: FTMS + c ESI Full ms [250.00-2000.00]

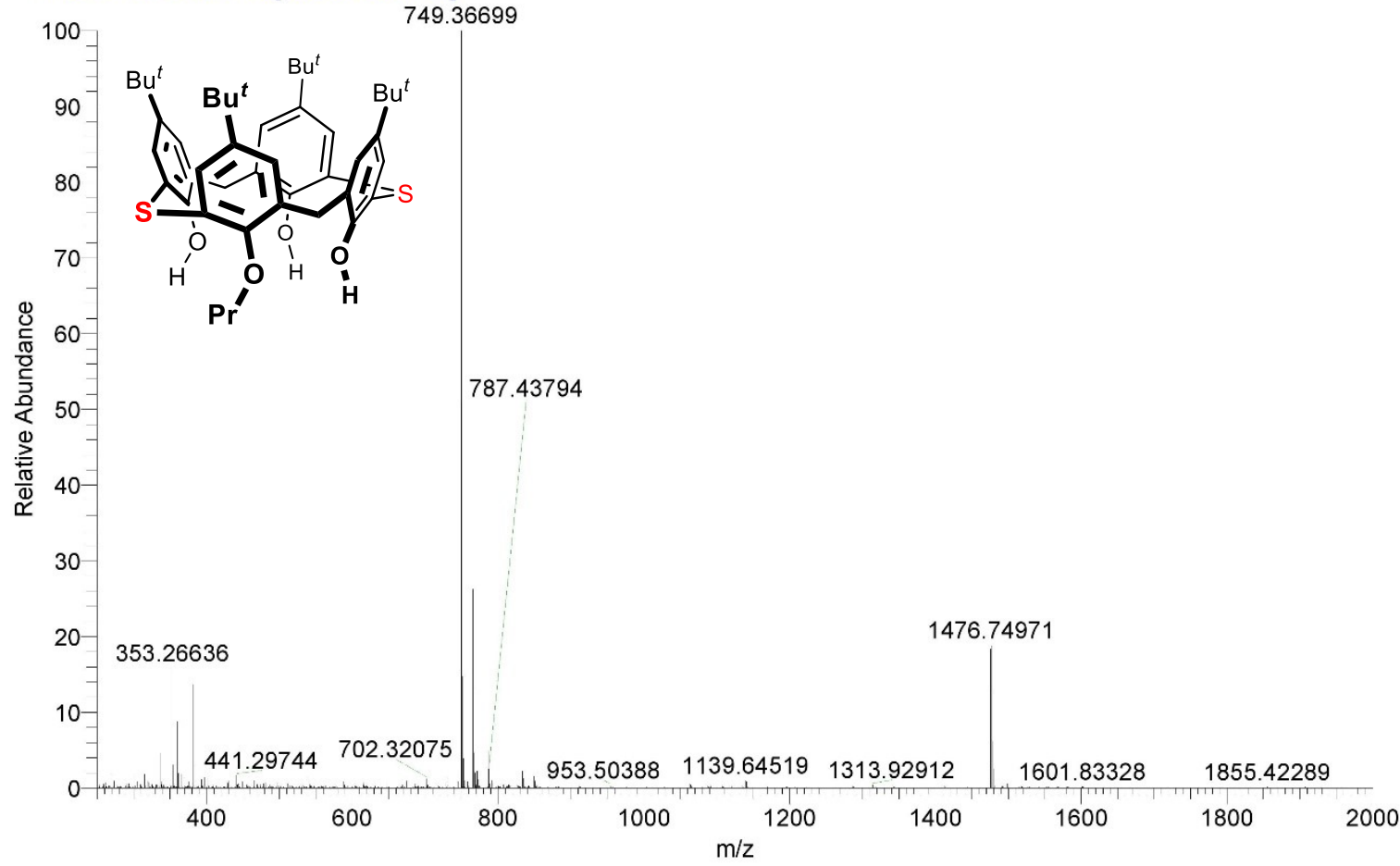

Figure S130. HRMS of compound $13\left(\mathrm{ESI}^{+}\right)$.

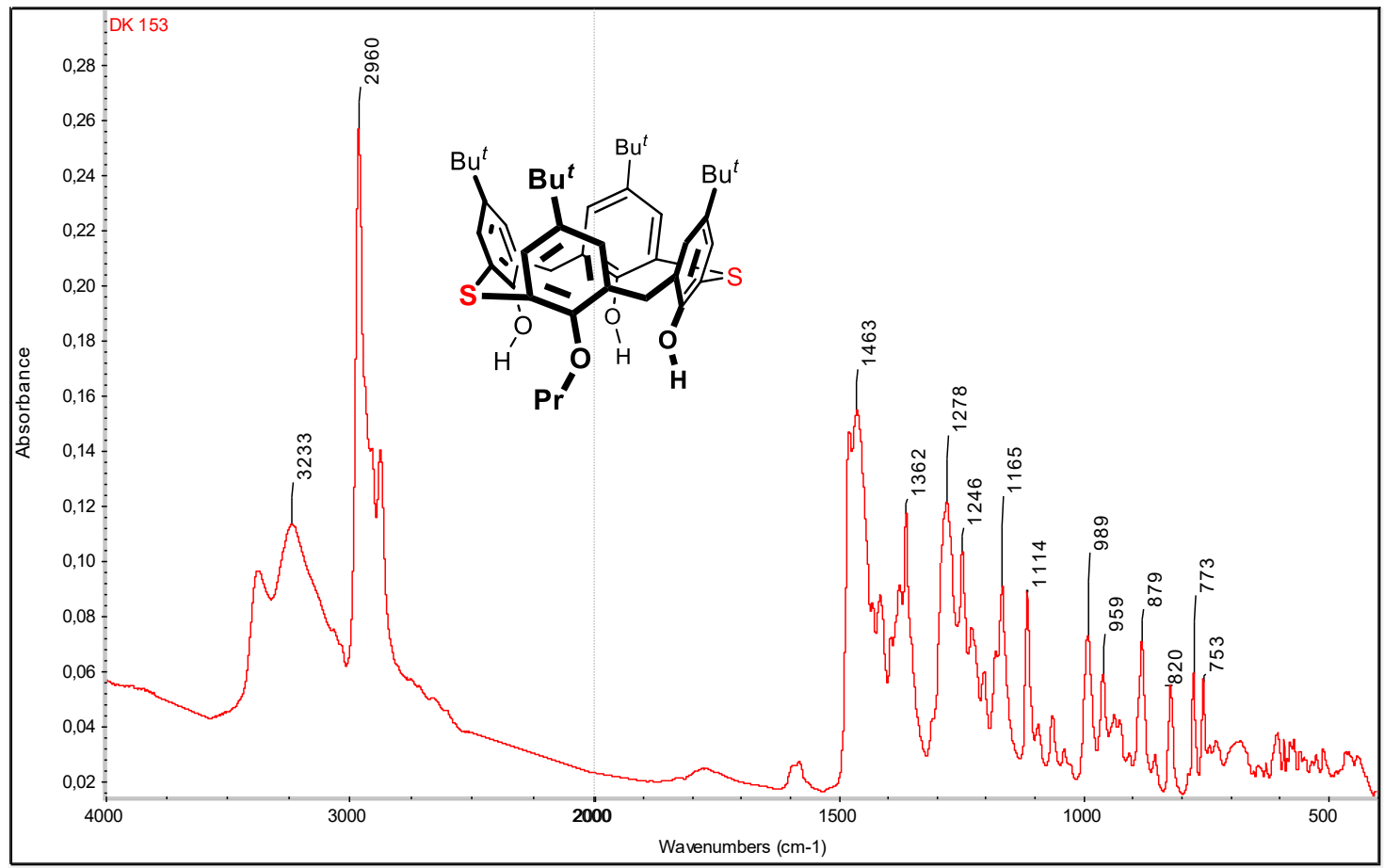

Figure 131. IR of compound 13 (ATR). 


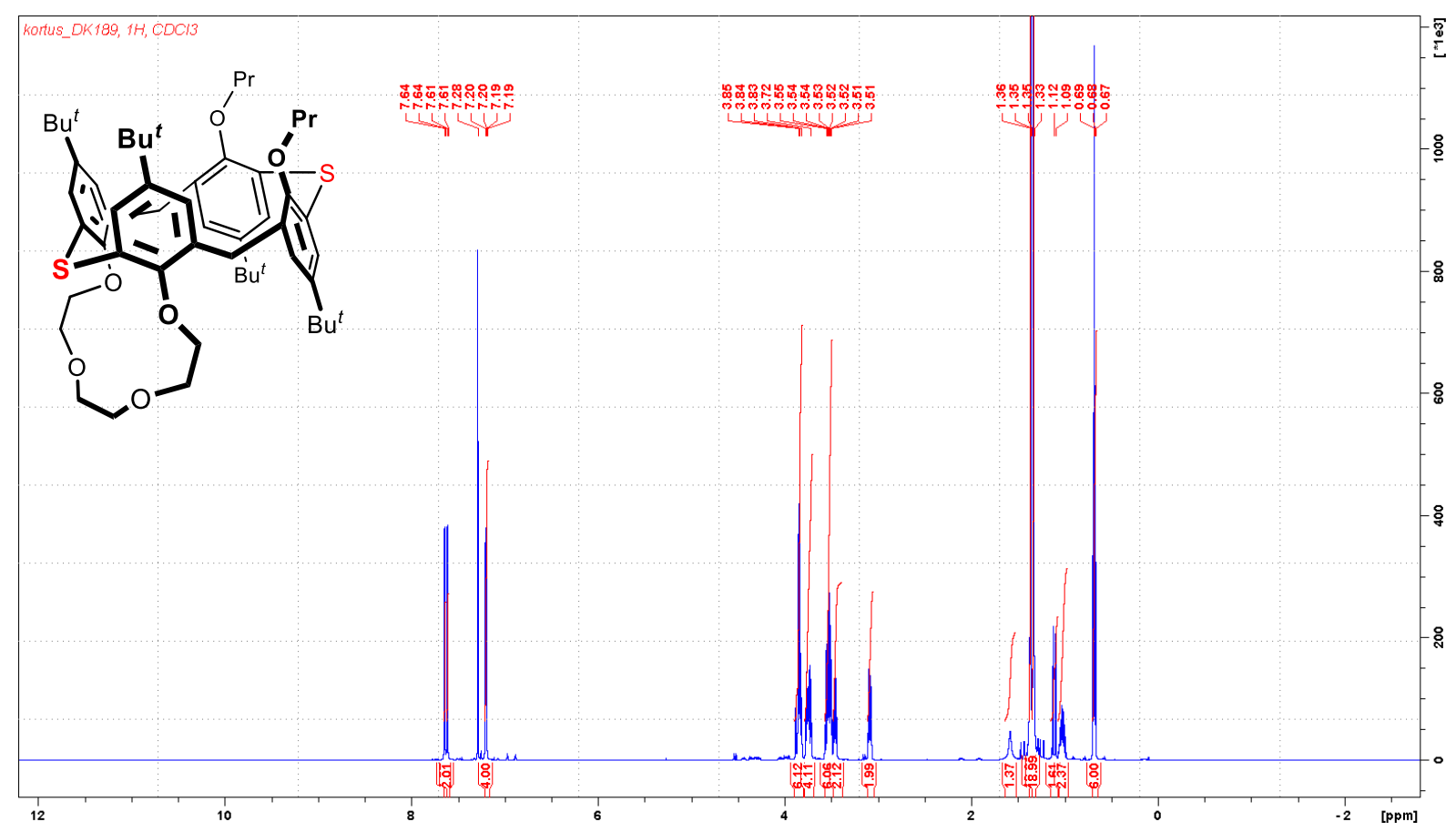

Figure S132. ${ }^{1} \mathrm{H}$ NMR of compound $14\left(\mathrm{CDCl}_{3}, 600 \mathrm{MHz}\right)$.

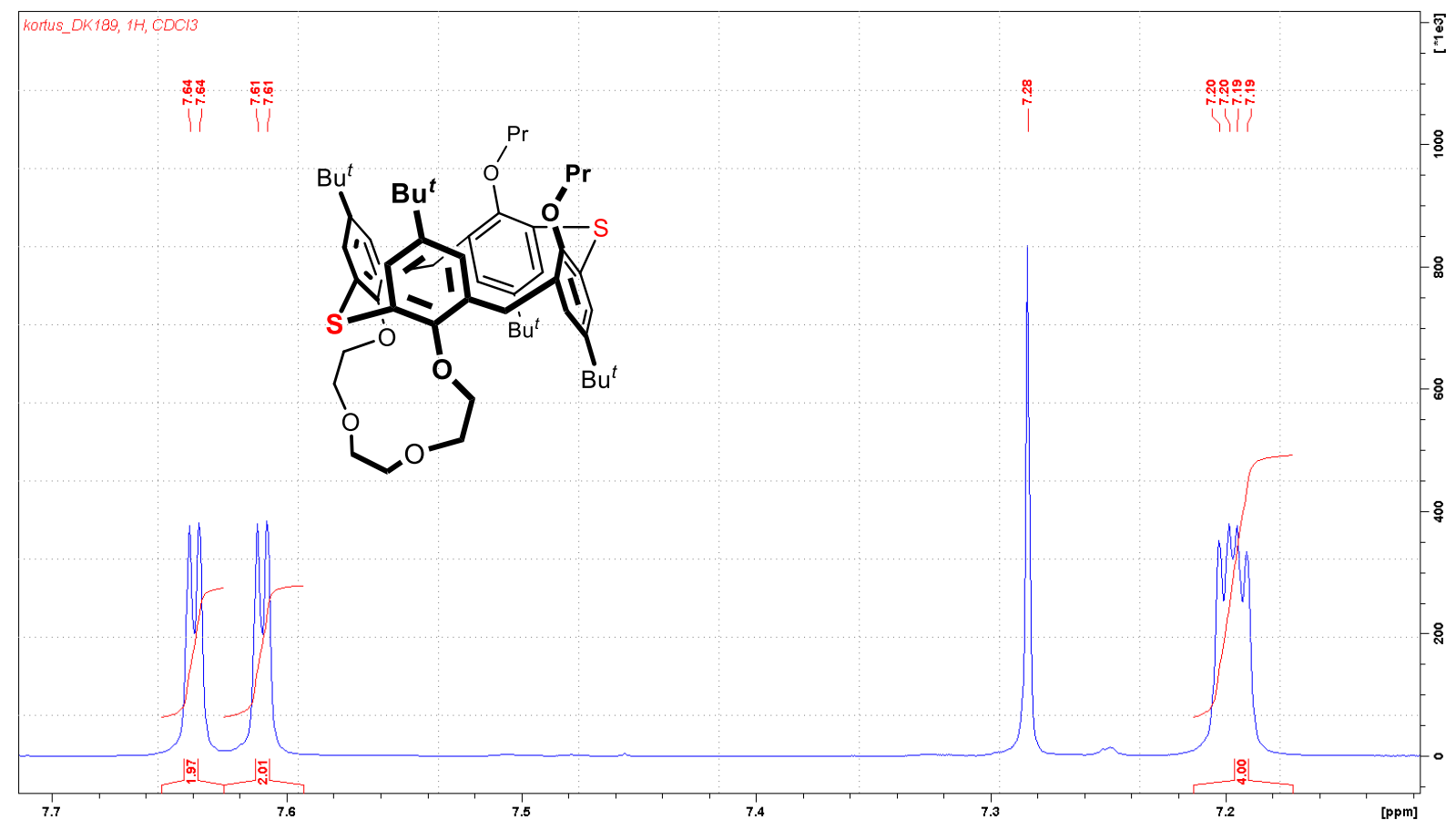

Figure S133. ${ }^{1} \mathrm{H}$ NMR of compound 14 , aromatic region $\left(\mathrm{CDCl}_{3}, 600 \mathrm{MHz}\right)$. 


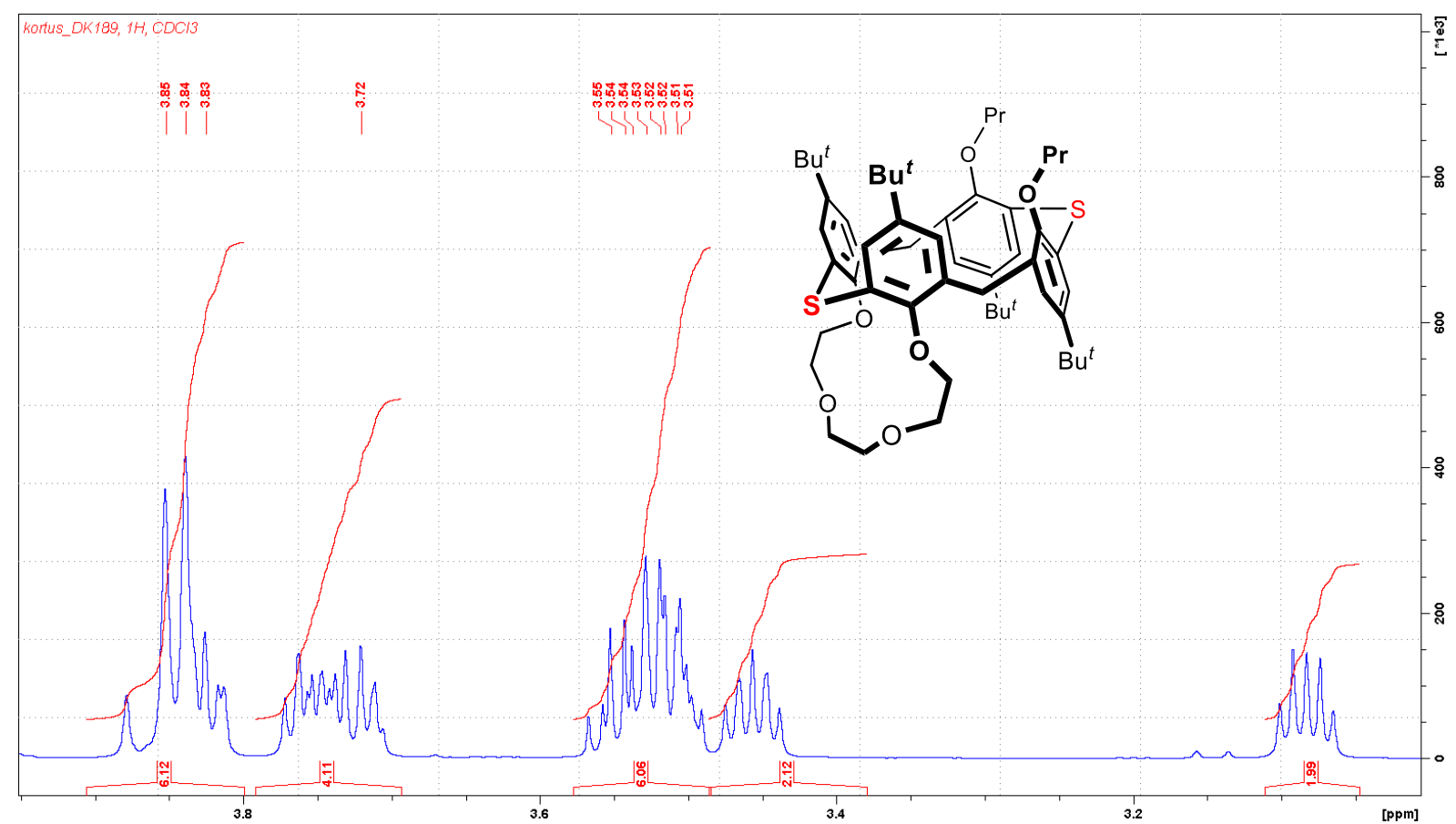

Figure S134. ${ }^{1} \mathrm{H}$ NMR of compound 14, bridge region $\left(\mathrm{CDCl}_{3}, 600 \mathrm{MHz}\right)$.

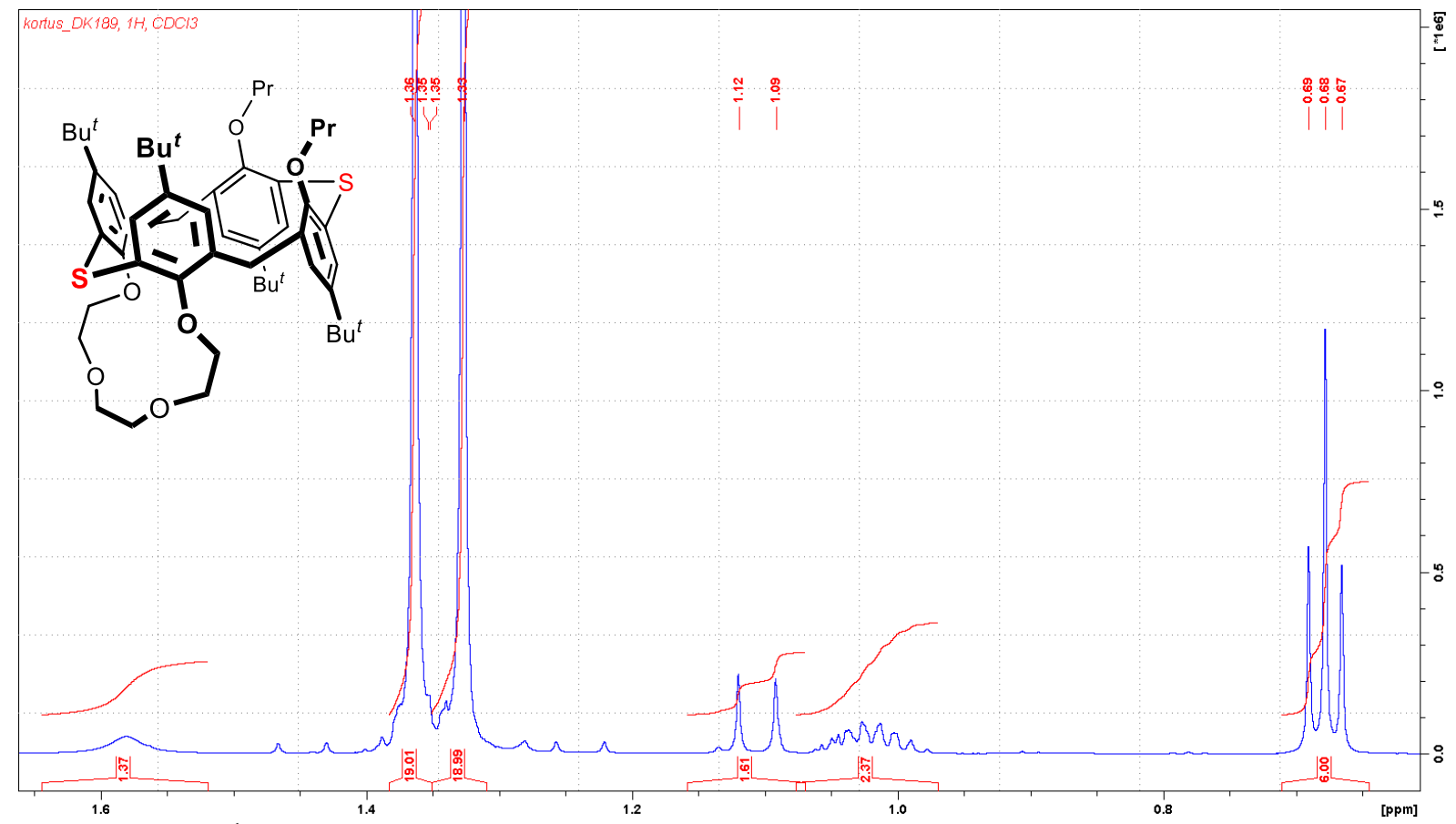

Figure S135. ${ }^{1} \mathrm{H}$ NMR of compound 14, aliphatic region $\left(\mathrm{CDCl}_{3}, 600 \mathrm{MHz}\right)$. 


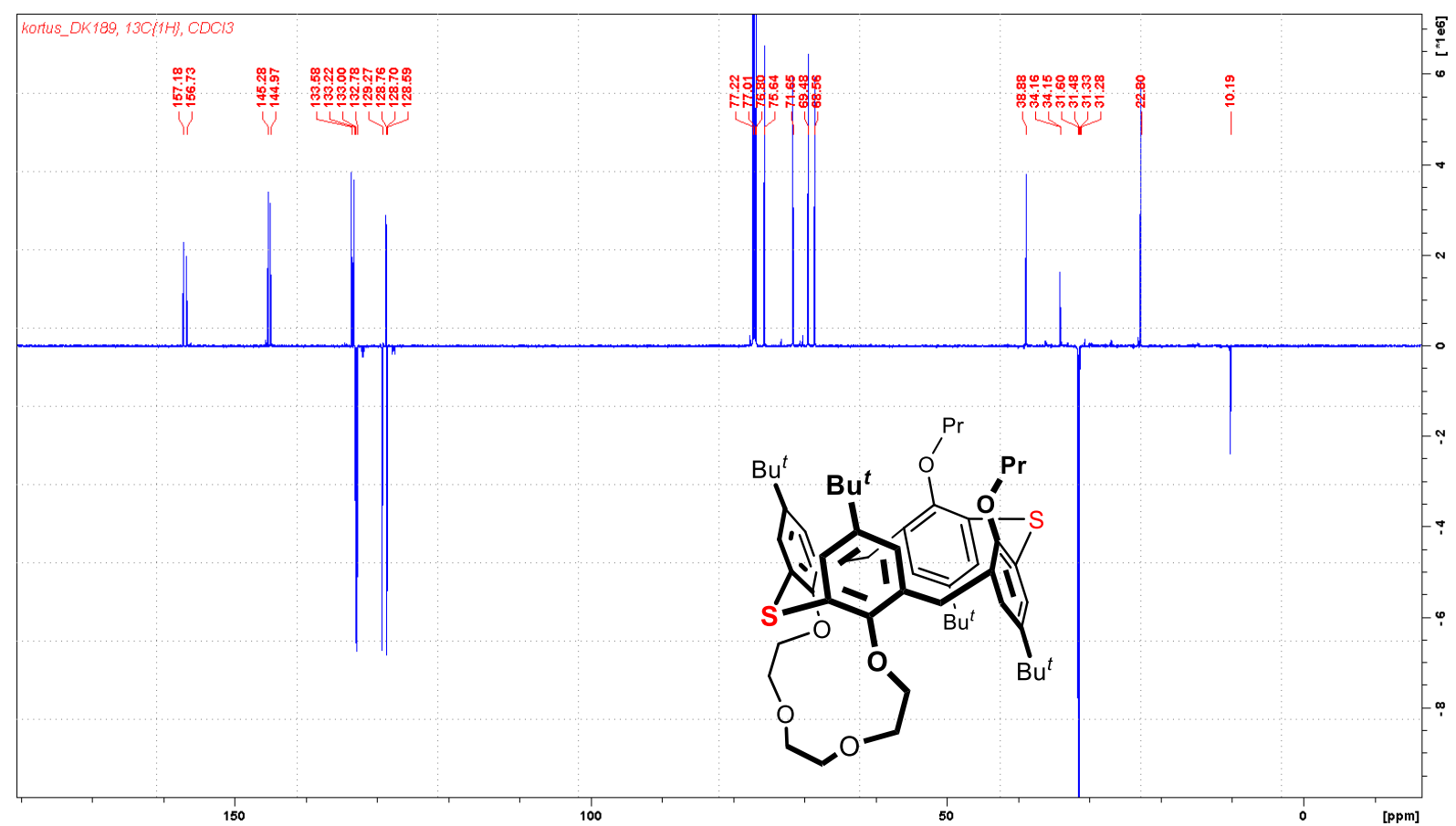

Figure S136. ${ }^{13} \mathrm{C}\left\{{ }^{1} \mathrm{H}\right\}$ NMR of compound $14\left(\mathrm{CDCl}_{3}, 151 \mathrm{MHz}\right)$.

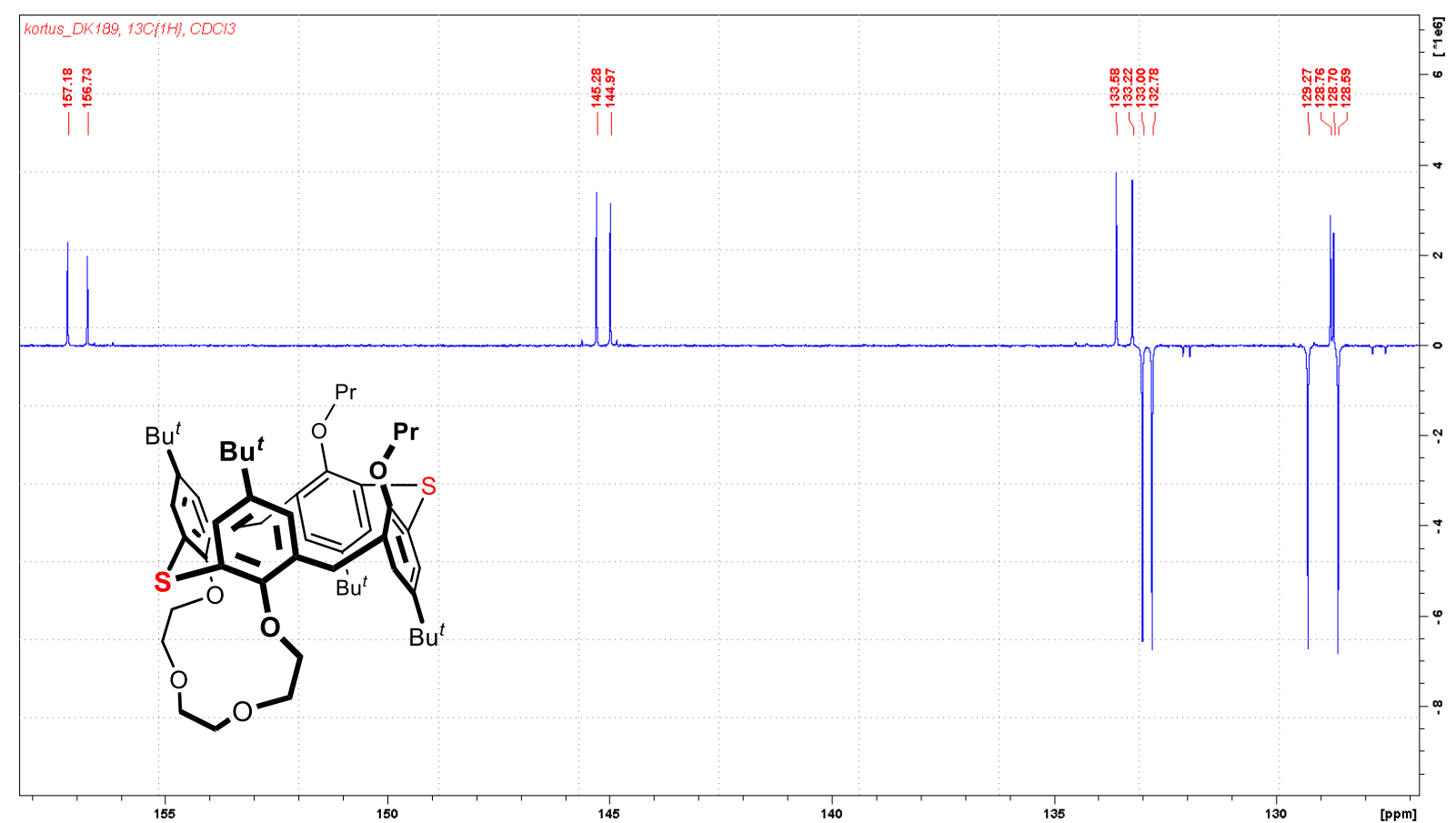

Figure S137. ${ }^{13} \mathrm{C}\left\{{ }^{1} \mathrm{H}\right\}$ NMR of compound 14, aromatic region $\left(\mathrm{CDCl}_{3}, 151 \mathrm{MHz}\right)$. 


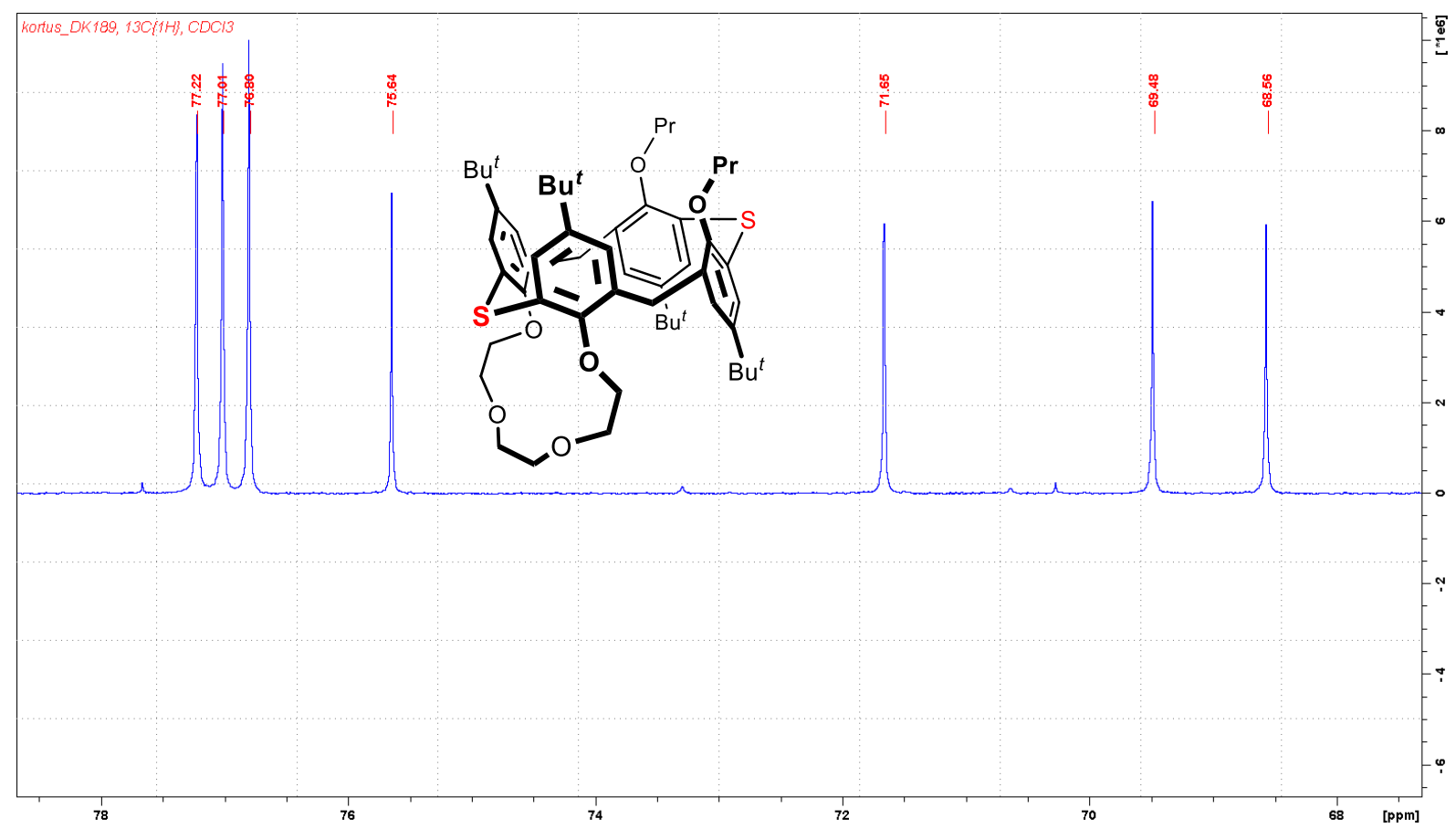

Figure S138. ${ }^{13} \mathrm{C}\left\{{ }^{1} \mathrm{H}\right\}$ NMR of compound 14, aliphatic region $\left(\mathrm{CDCl}_{3}, 151 \mathrm{MHz}\right)$.

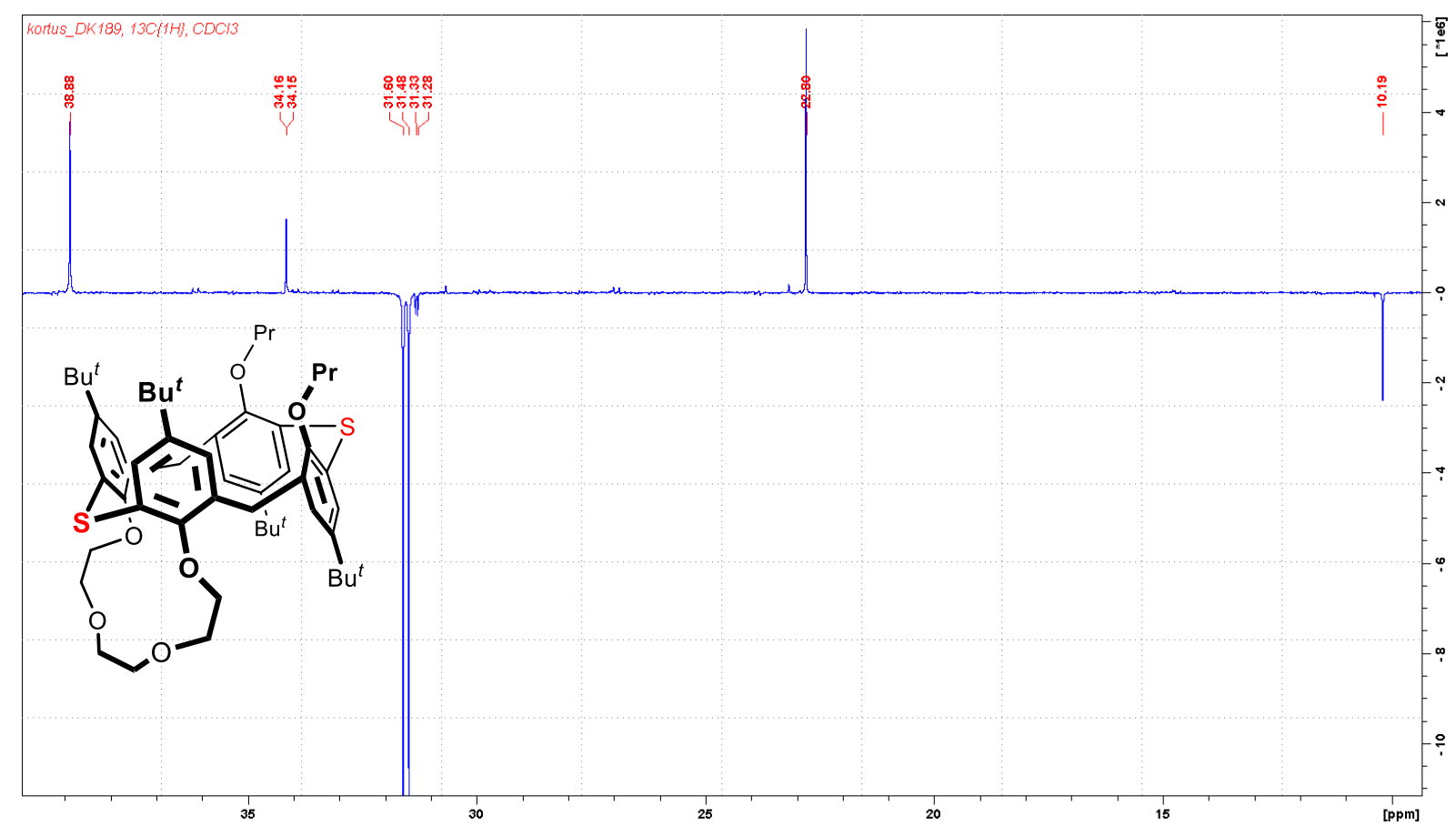

Figure S139. ${ }^{13} \mathrm{C}\left\{{ }^{1} \mathrm{H}\right\}$ NMR of compound 14, aliphatic region $\left(\mathrm{CDCl}_{3}, 151 \mathrm{MHz}\right)$. 


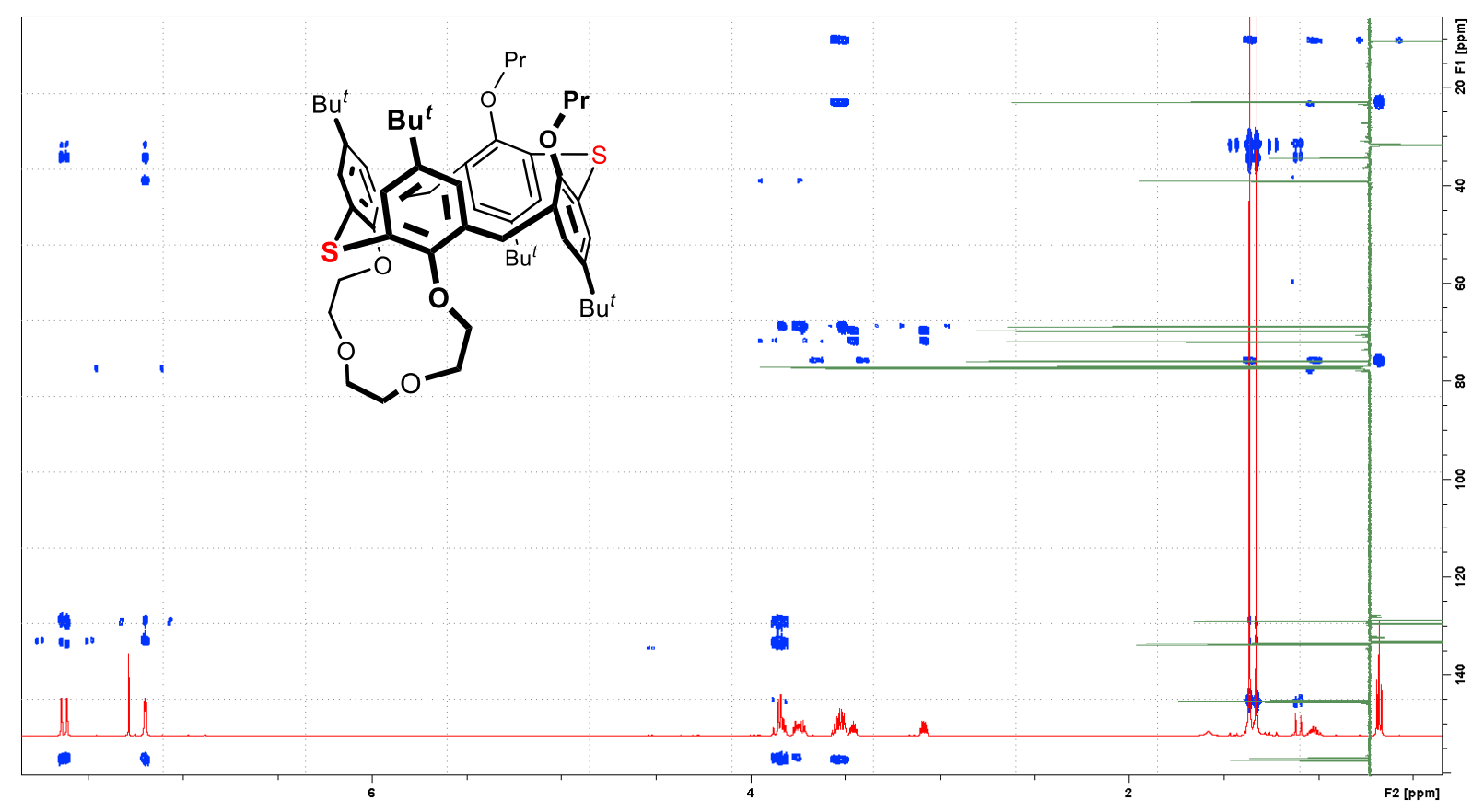

Figure S140. HMBC NMR of compound $14\left(\mathrm{CDCl}_{3}\right)$.

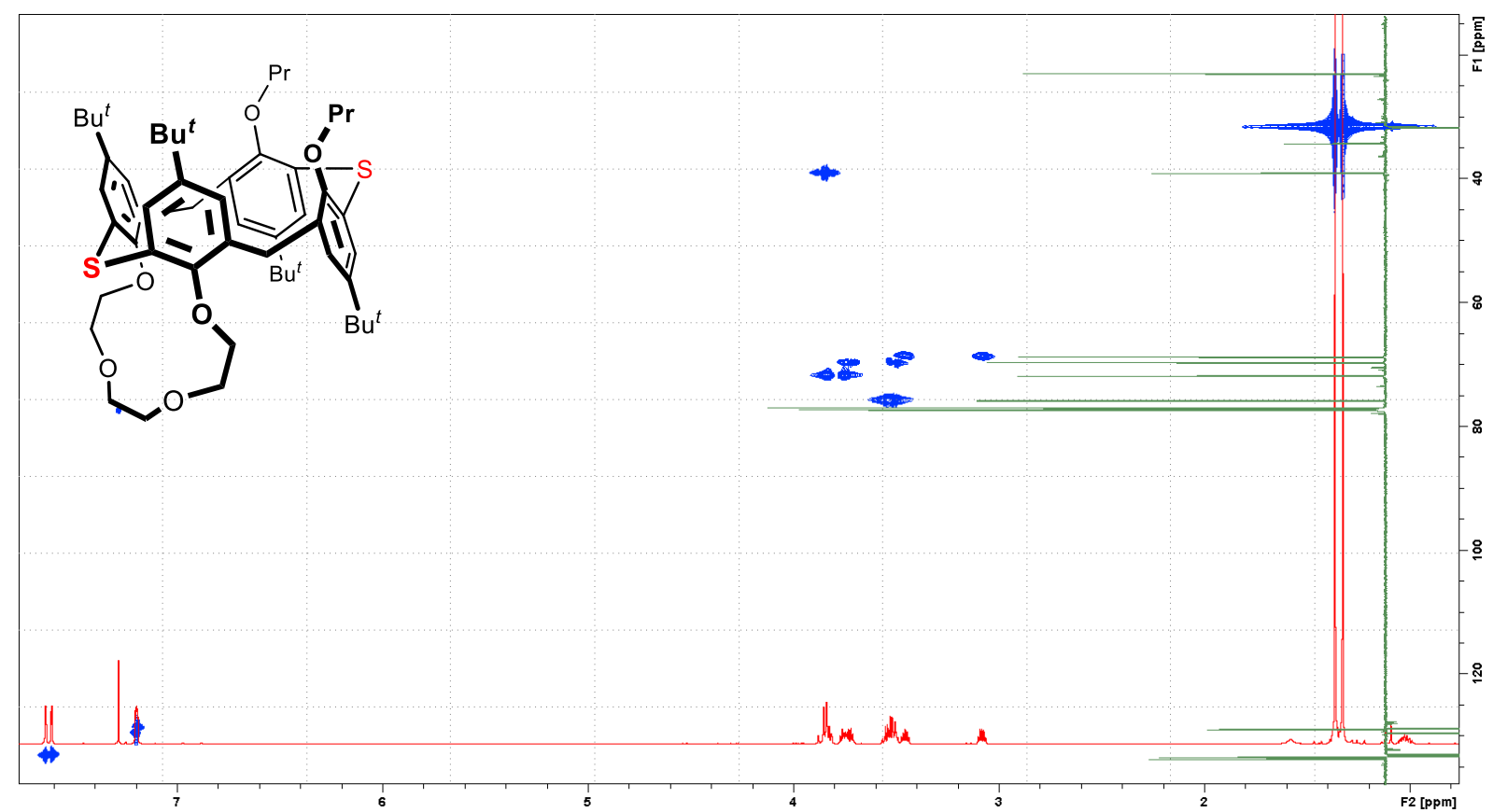

Figure S141. HMQC NMR of compound $14\left(\mathrm{CDCl}_{3}\right)$. 


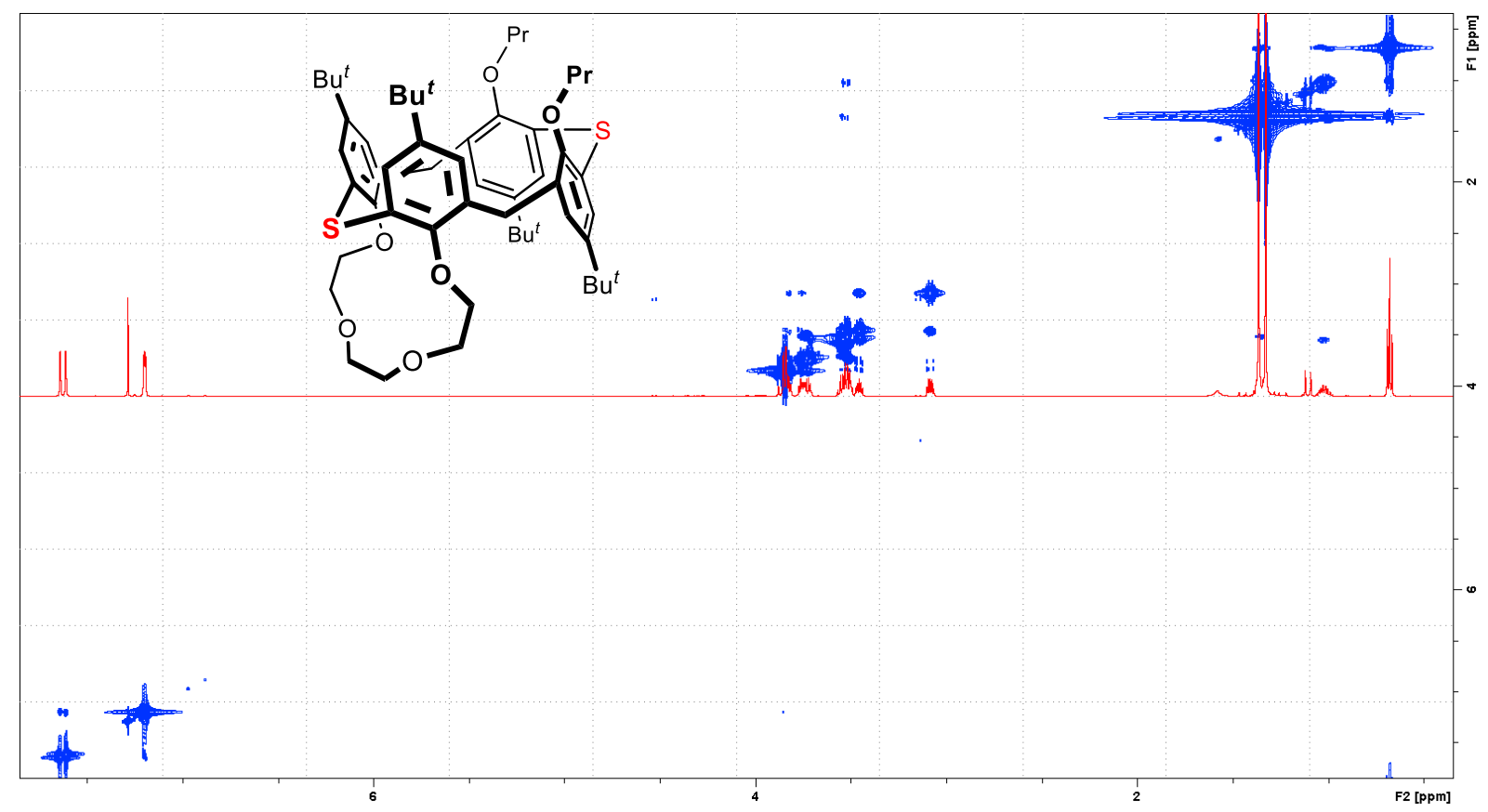

Figure S142. COSY NMR of compound $14\left(\mathrm{CDCl}_{3}\right)$.

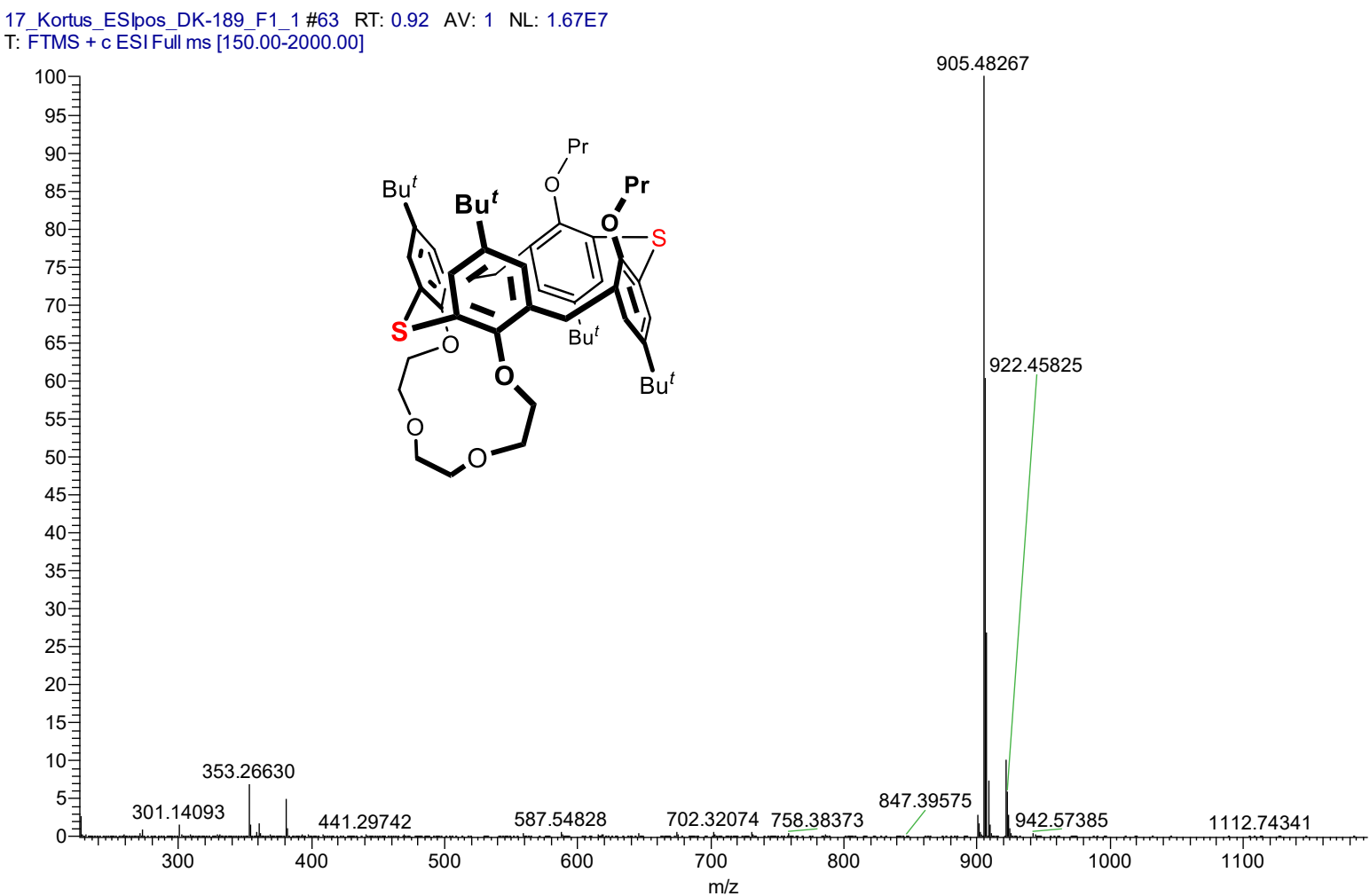

Figure S143. HRMS of compound $14\left(\mathrm{ESI}^{+}\right)$. 


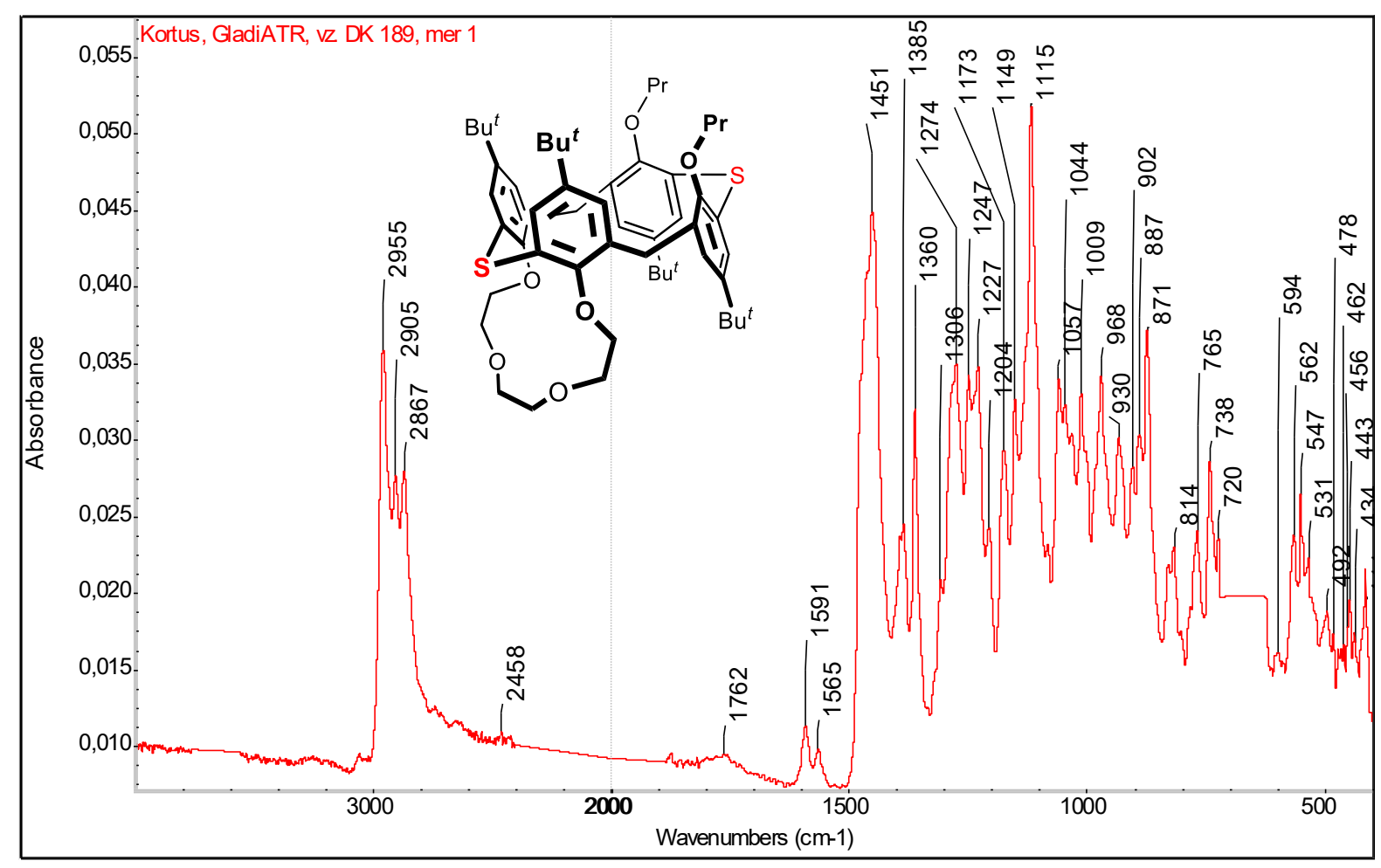

Figure S144. IR of compound 14 (ATR). 


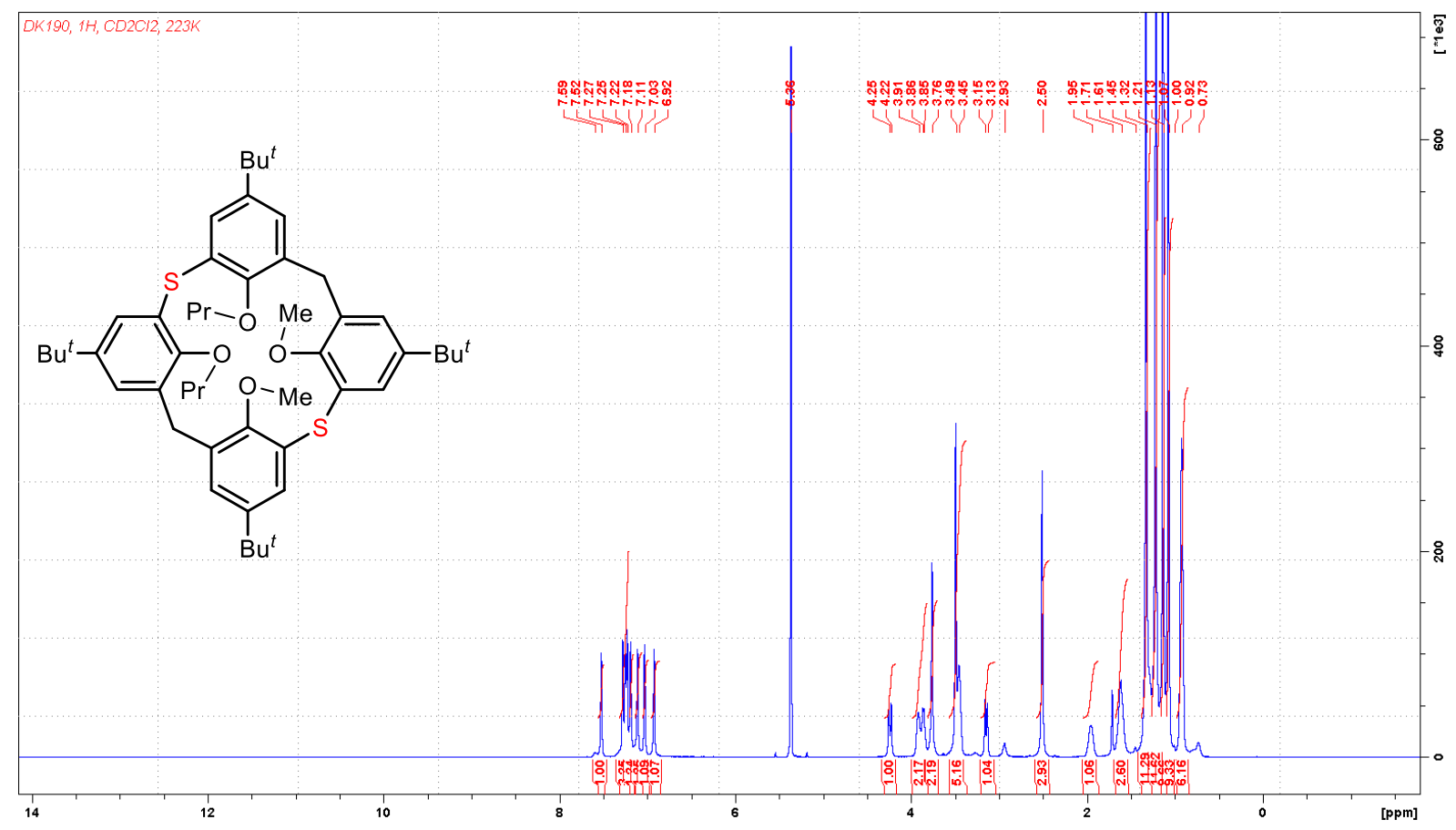

Figure S145. ${ }^{1} \mathrm{H}$ NMR of compound $15\left(\mathrm{CD}_{2} \mathrm{Cl}_{2}, 223 \mathrm{~K}, 600 \mathrm{MHz}\right)$.

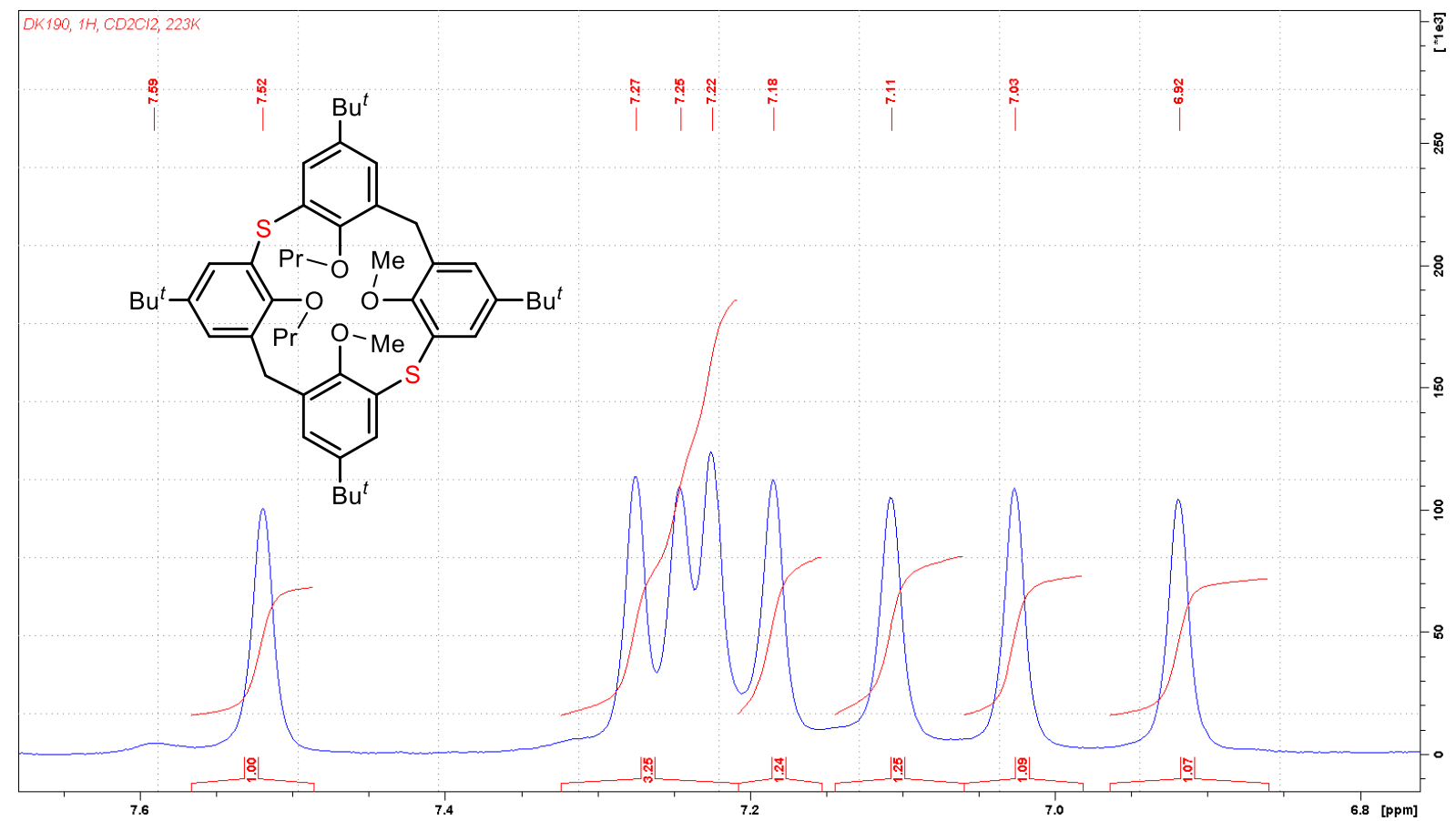

Figure S146. ${ }^{1} \mathrm{H}$ NMR of compound 15, aromatic region $\left(\mathrm{CD}_{2} \mathrm{Cl}_{2}, 223 \mathrm{~K}, 600 \mathrm{MHz}\right)$. 


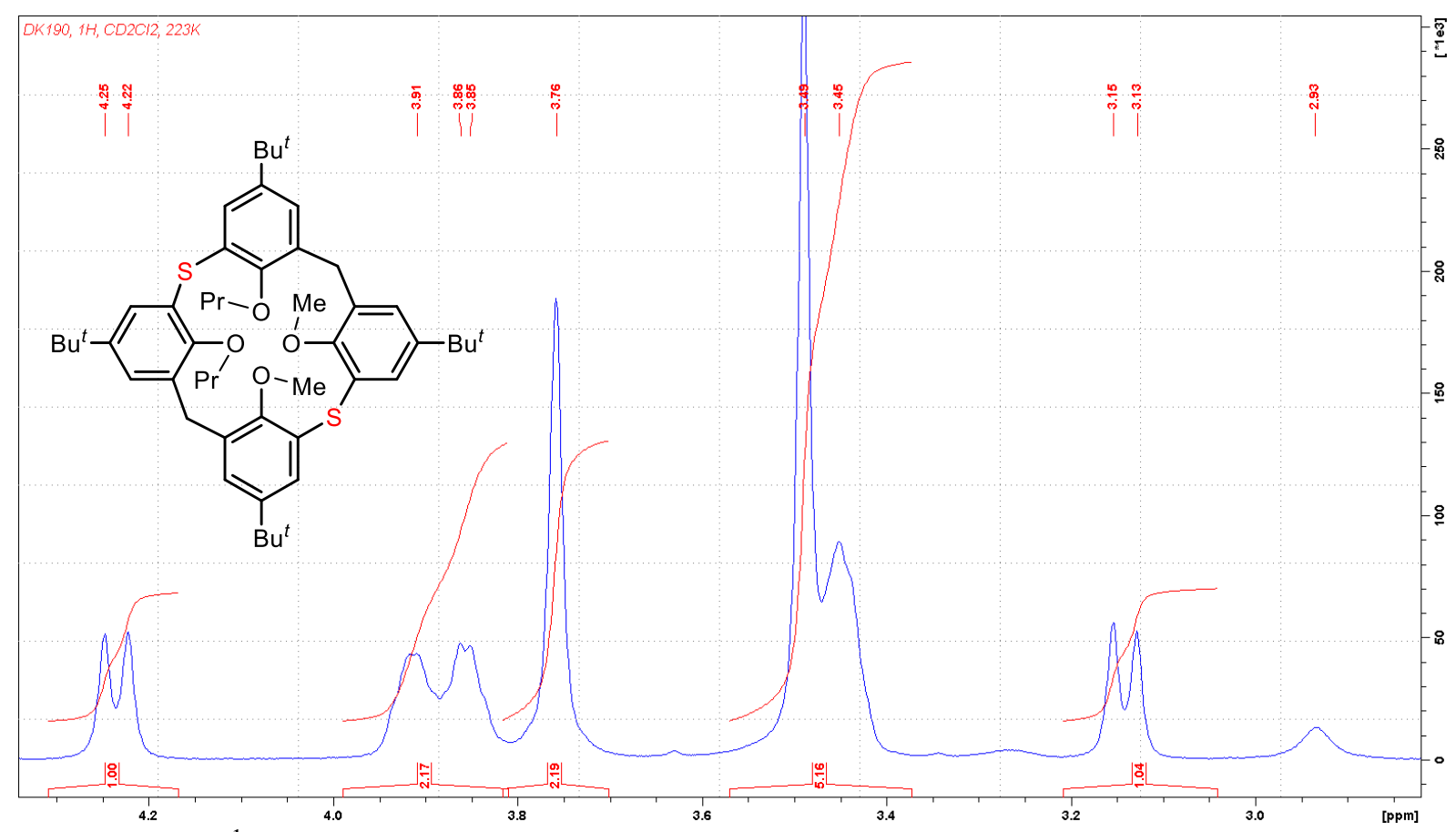

Figure S147. ${ }^{1} \mathrm{H}$ NMR of compound 15, bridge region $\left(\mathrm{CD}_{2} \mathrm{Cl}_{2}, 223 \mathrm{~K}, 600 \mathrm{MHz}\right)$.

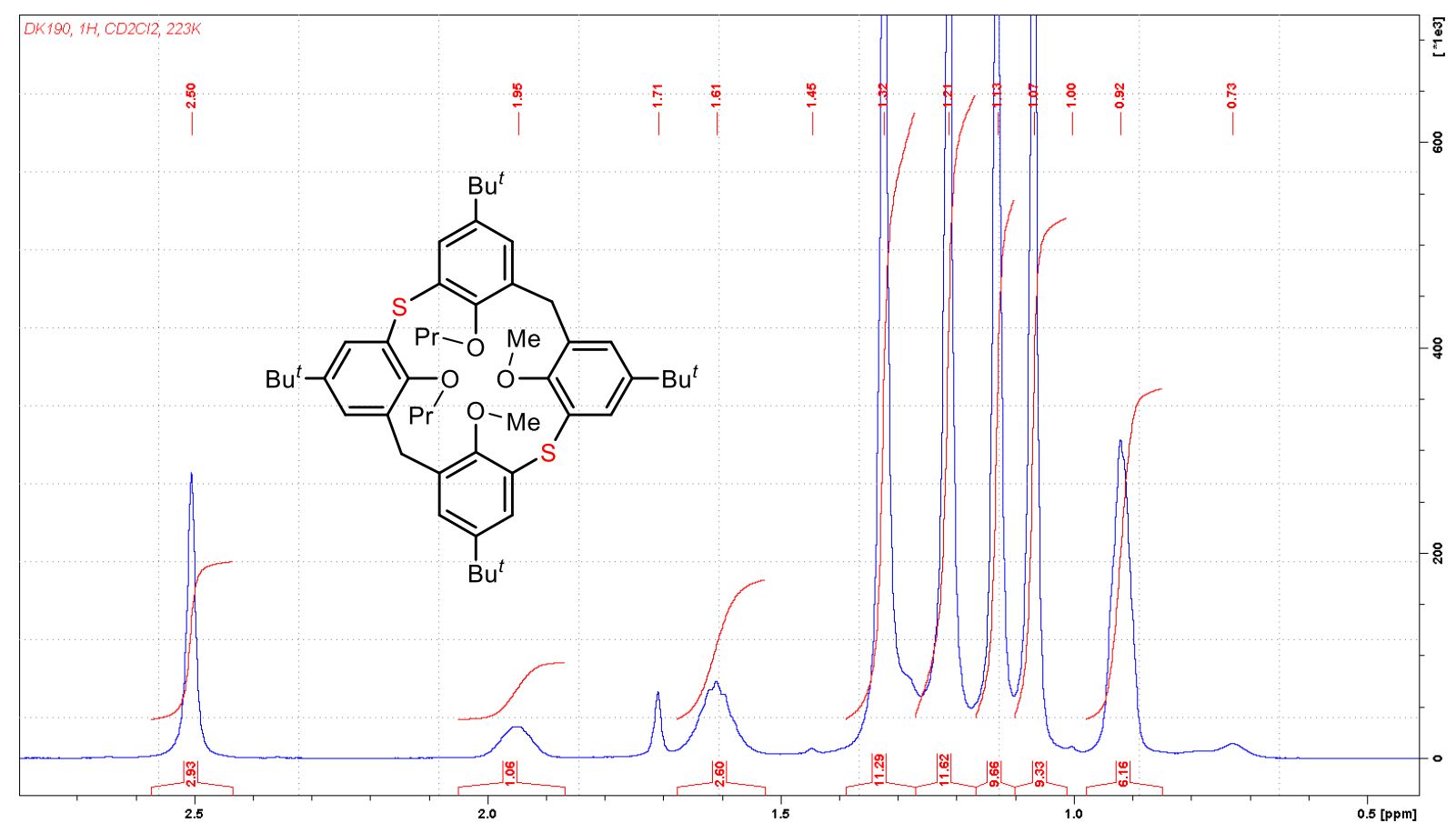

Figure S148. ${ }^{1} \mathrm{H}$ NMR of compound 15, aliphatic region $\left(\mathrm{CD}_{2} \mathrm{Cl}_{2}, 223 \mathrm{~K}, 600 \mathrm{MHz}\right)$. 


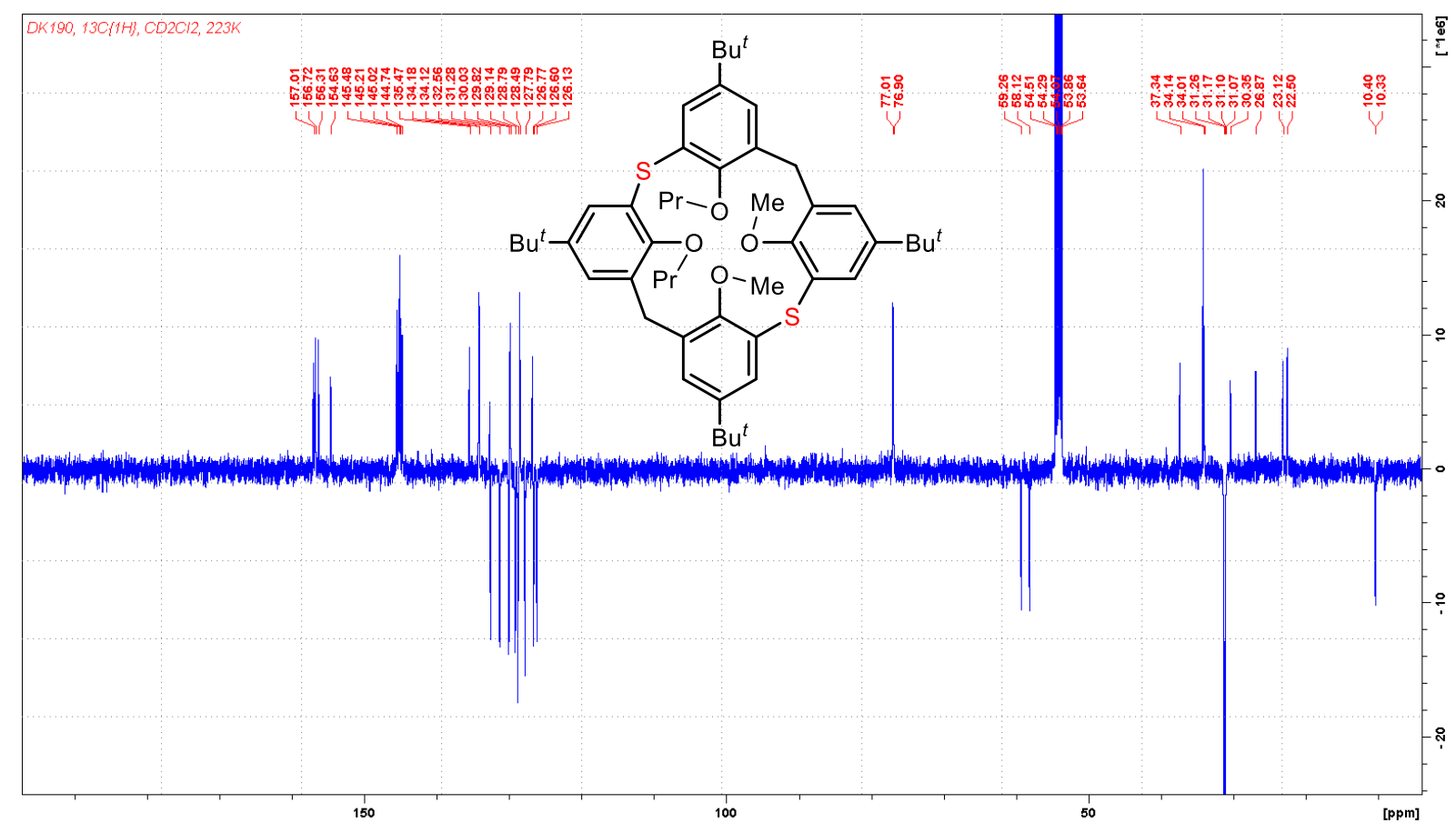

Figure S149. ${ }^{13} \mathrm{C}\left\{{ }^{1} \mathrm{H}\right\}$ NMR of compound $15\left(\mathrm{CD}_{2} \mathrm{Cl}_{2}, 223 \mathrm{~K}, 151 \mathrm{MHz}\right)$.

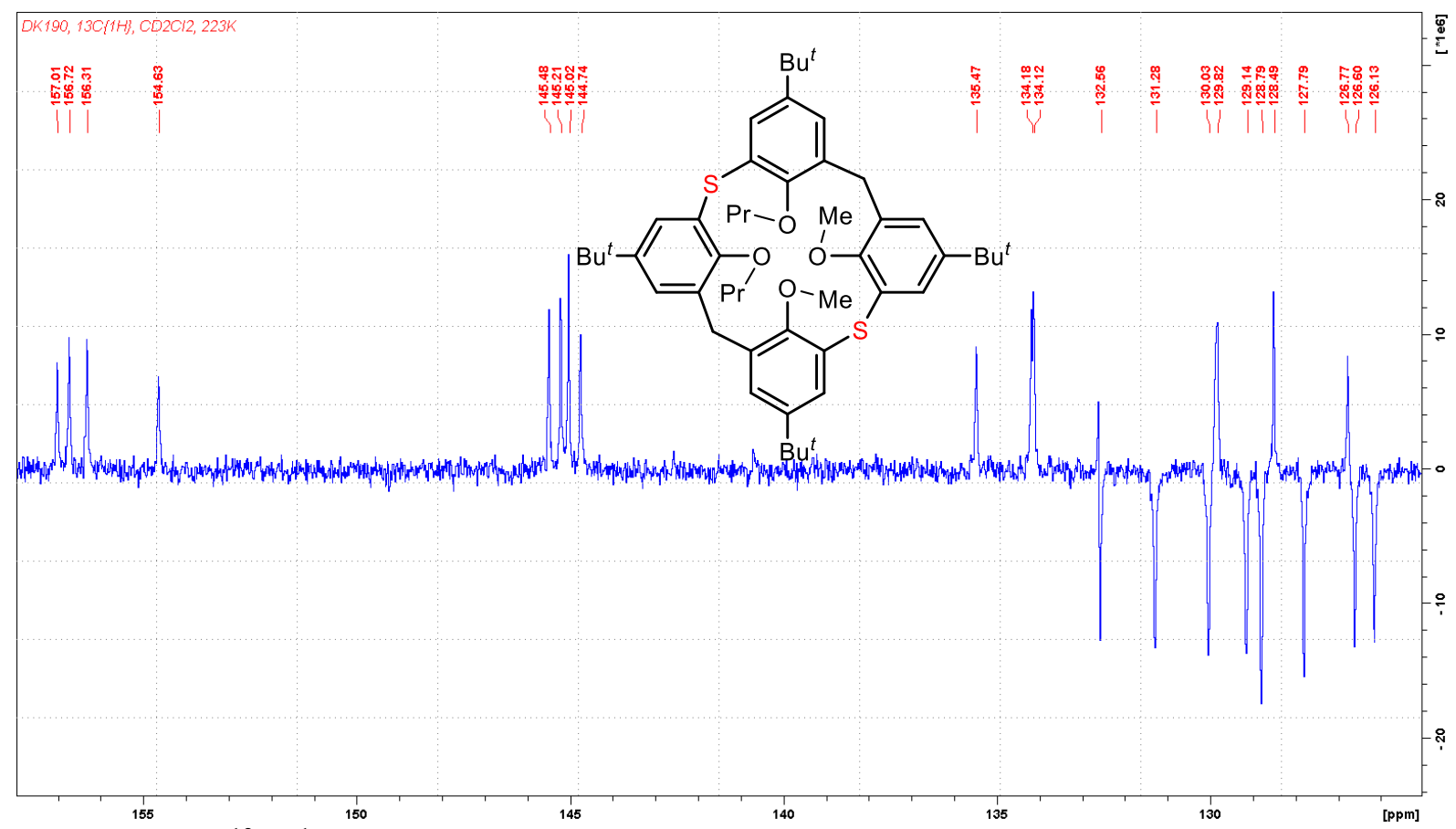

Figure S150. ${ }^{13} \mathrm{C}\left\{{ }^{1} \mathrm{H}\right\}$ NMR of compound 15, aromatic region $\left(\mathrm{CD}_{2} \mathrm{Cl}_{2}, 223 \mathrm{~K}, 151 \mathrm{MHz}\right)$. 


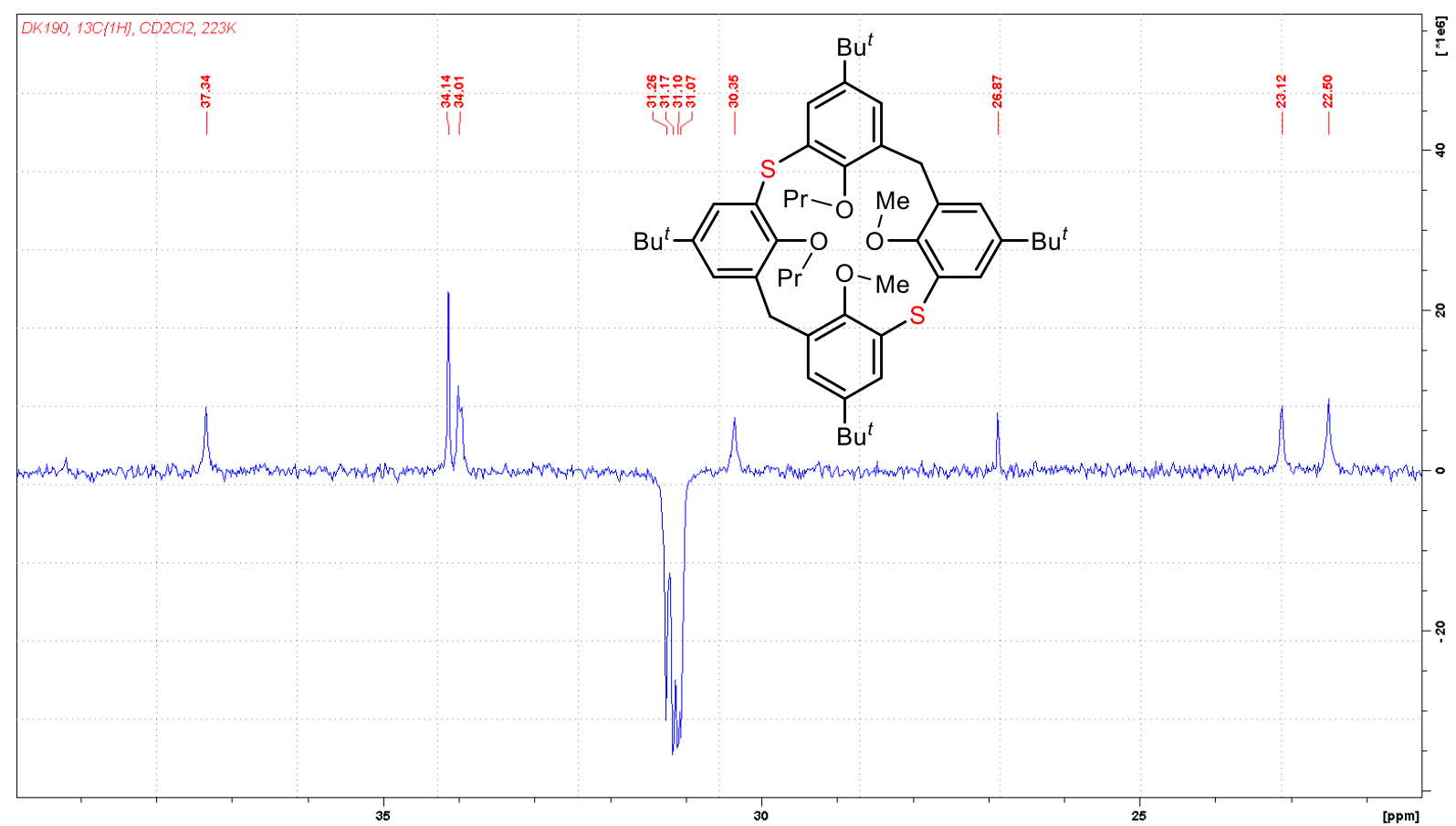

Figure S151. ${ }^{13} \mathrm{C}\left\{{ }^{1} \mathrm{H}\right\}$ NMR of compound 15, aliphatic region $\left(\mathrm{CD}_{2} \mathrm{Cl}_{2}, 223 \mathrm{~K}, 151 \mathrm{MHz}\right)$.

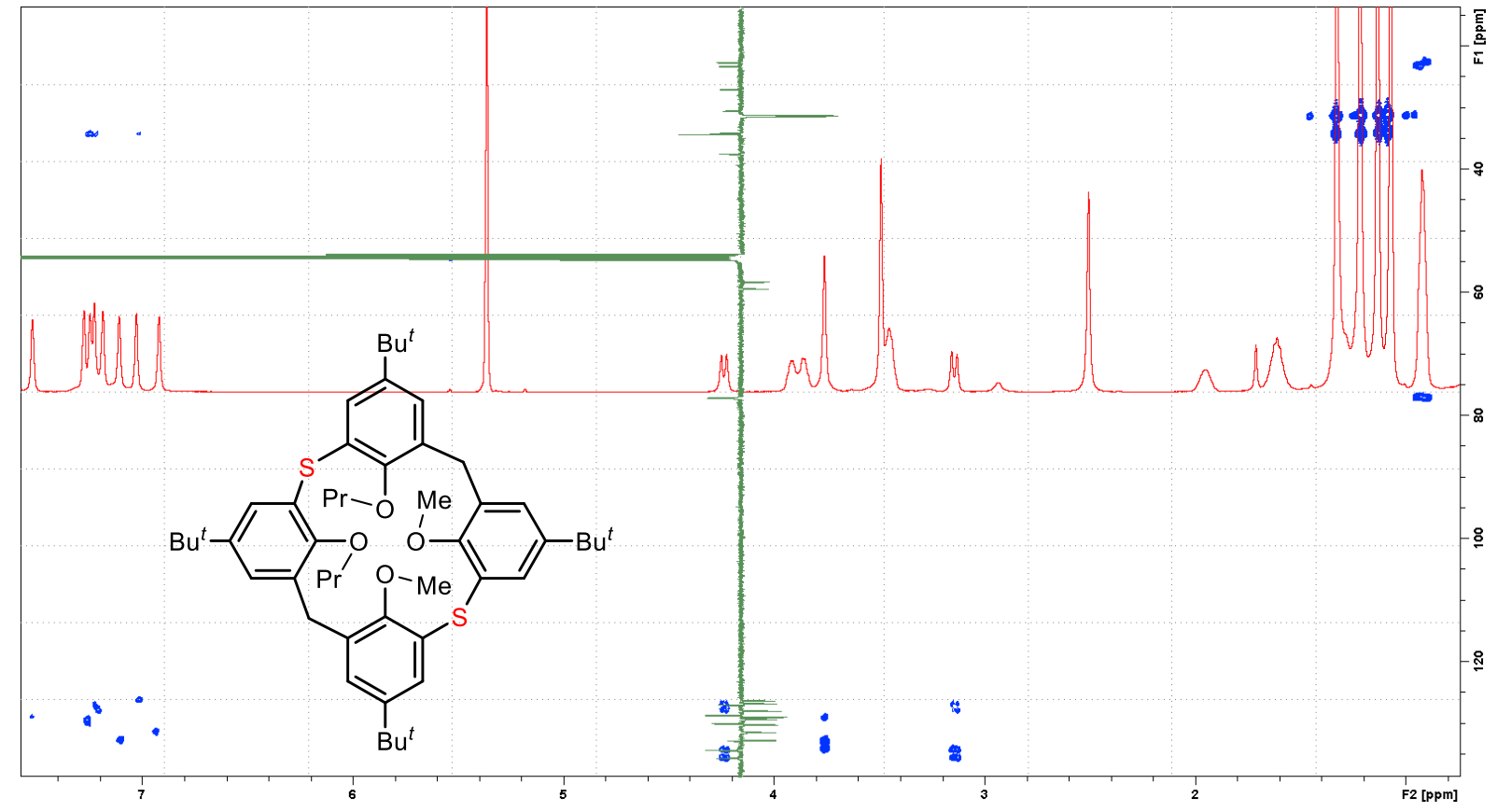

Figure S152. HMBC NMR of compound $15\left(\mathrm{CD}_{2} \mathrm{Cl}_{2}, 223 \mathrm{~K}\right)$. 


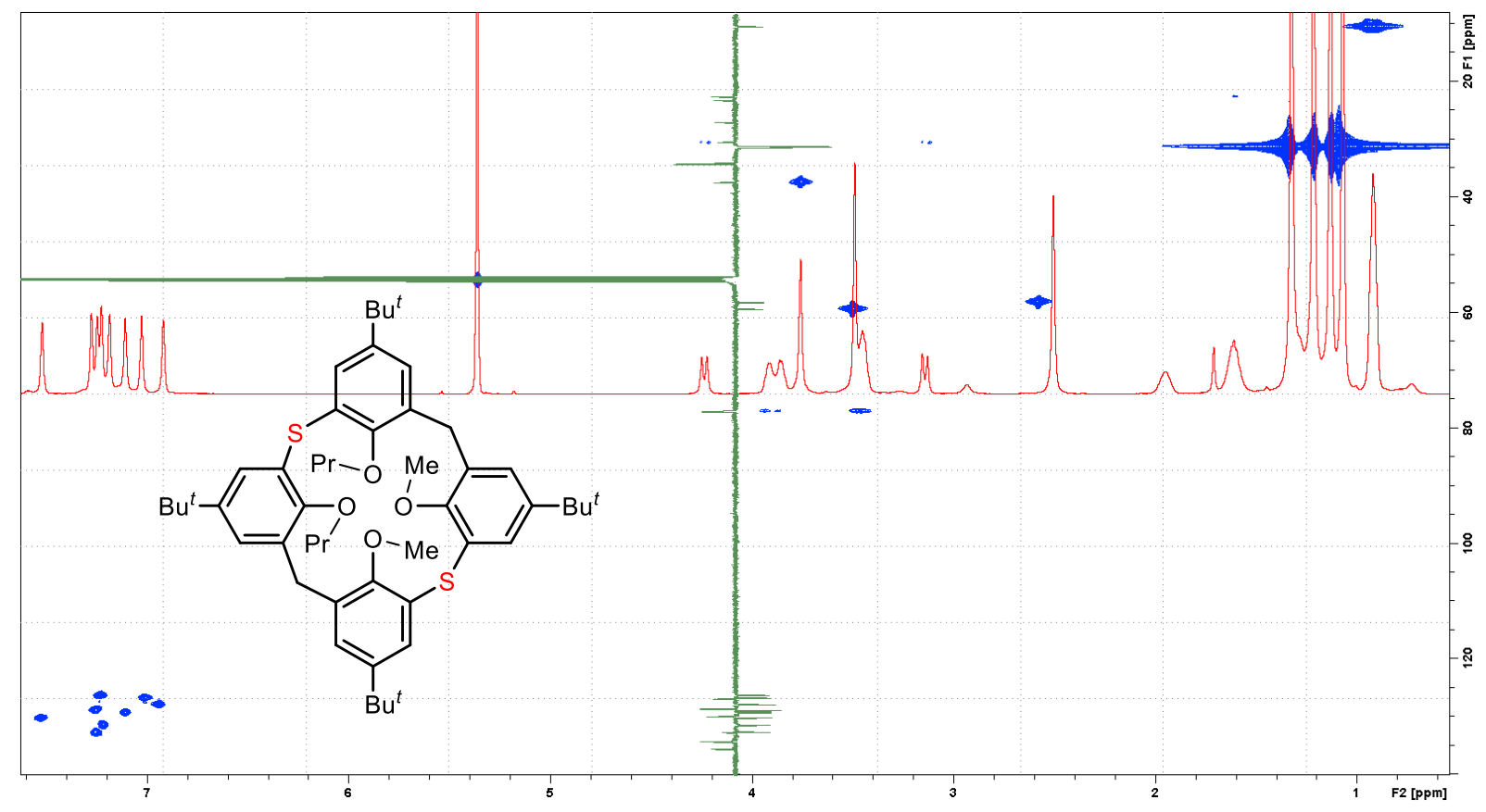

Figure S153. HMQC NMR of compound $15\left(\mathrm{CD}_{2} \mathrm{Cl}_{2}, 223 \mathrm{~K}\right)$.

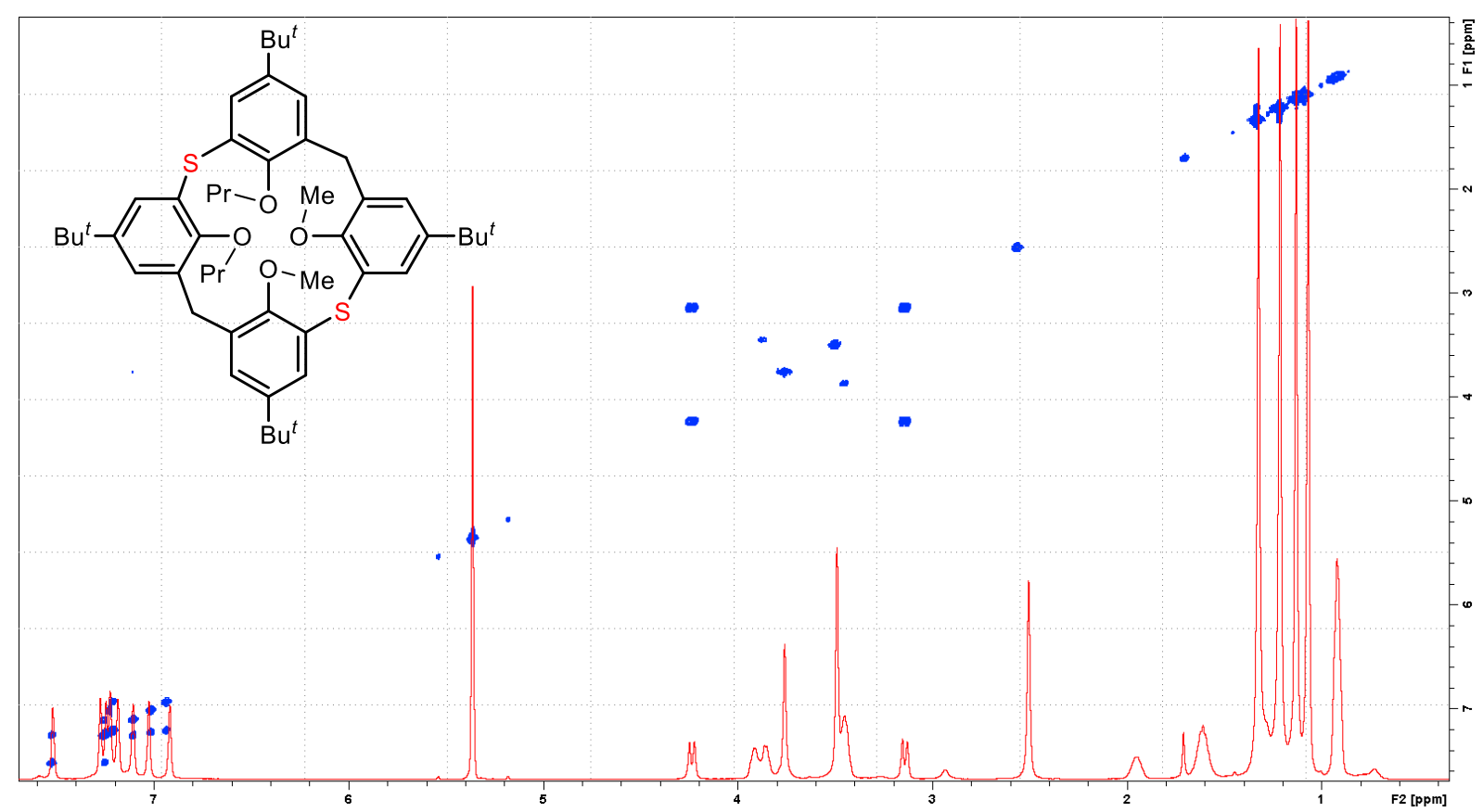

Figure S154. COSY NMR of compound $15\left(\mathrm{CD}_{2} \mathrm{Cl}_{2}, 223 \mathrm{~K}\right)$. 


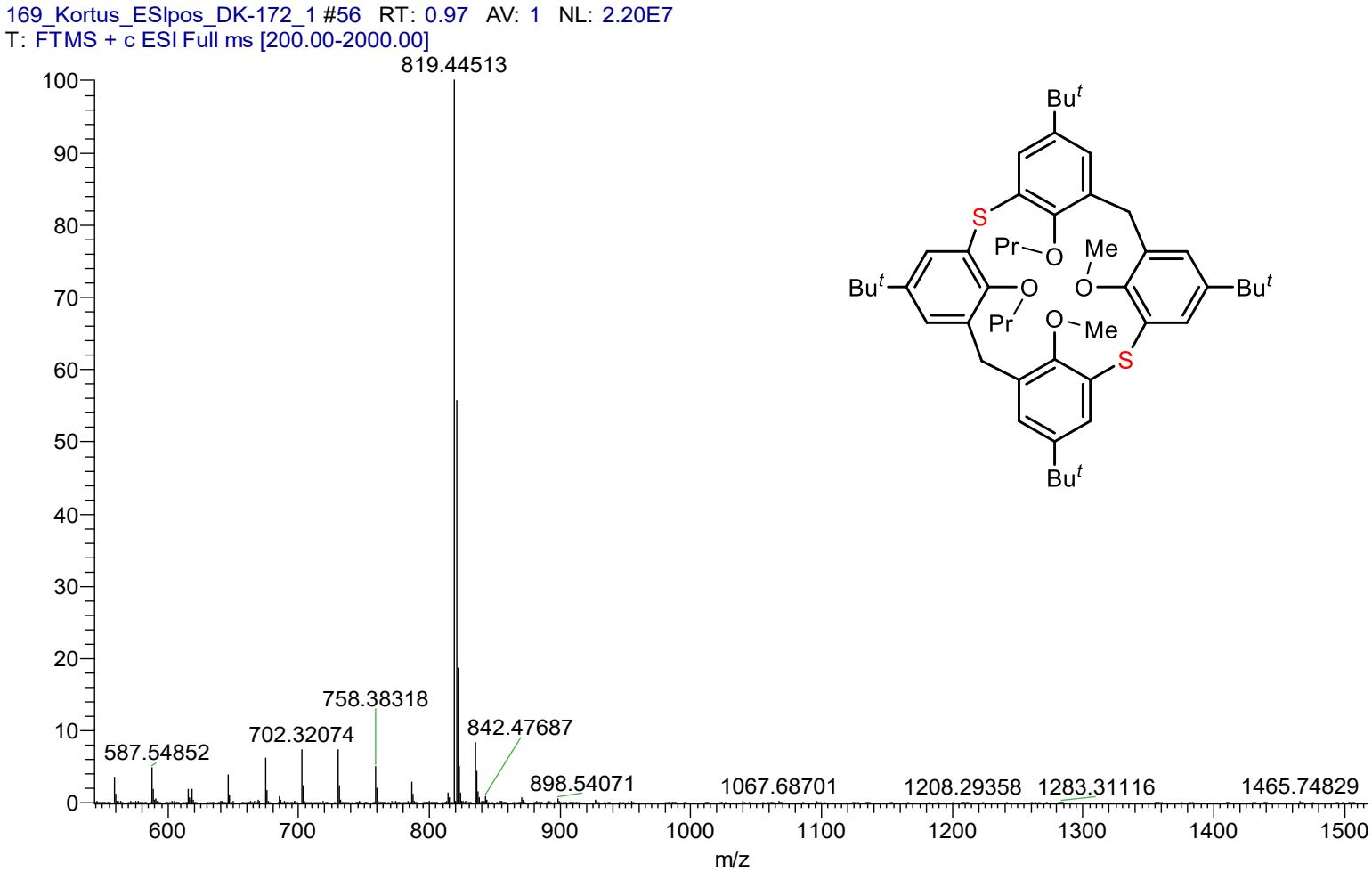

Figure S155. HRMS of compound $15\left(\mathrm{ESI}^{+}\right)$.

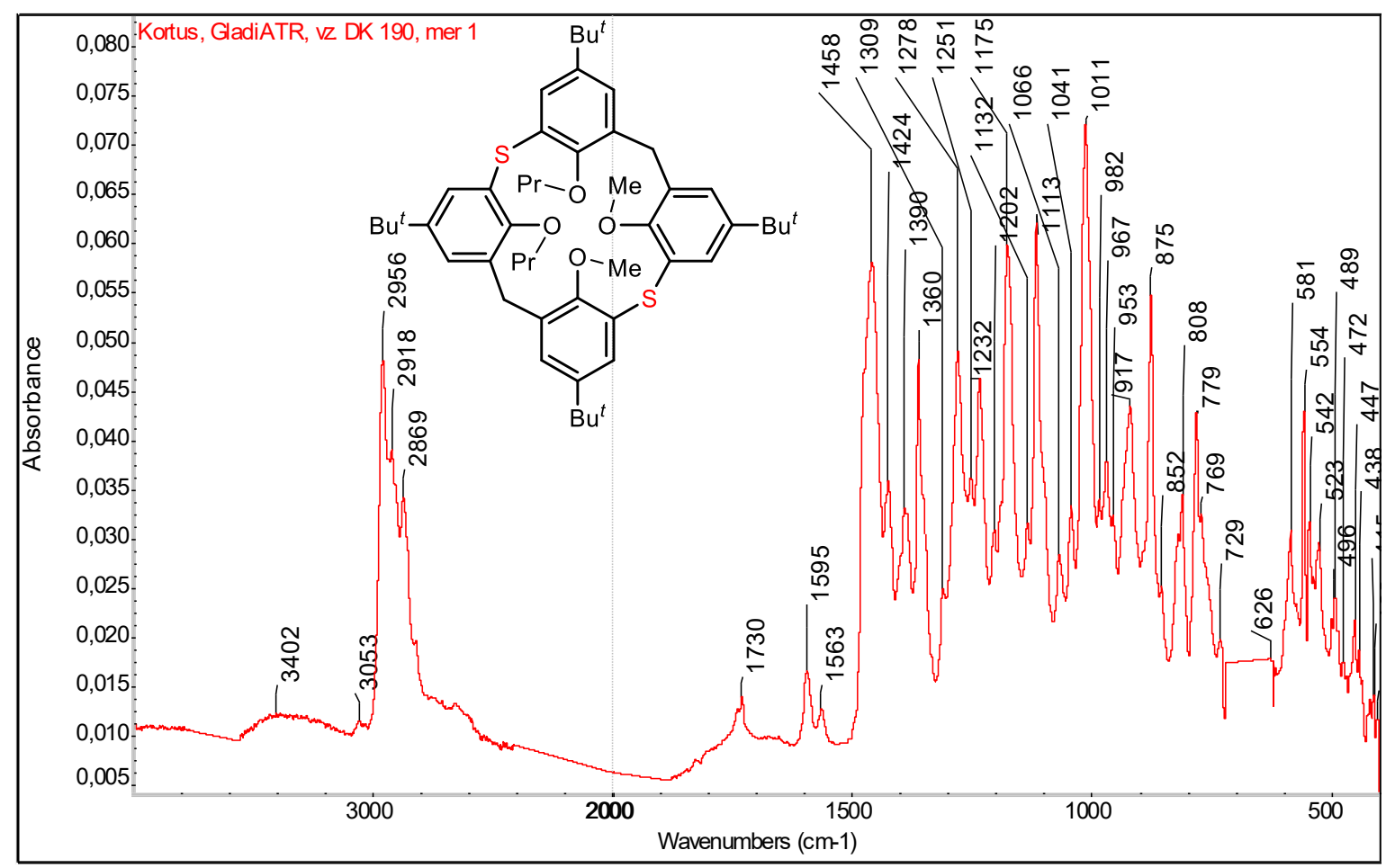

Figure S156. IR of compound 15 (ATR). 


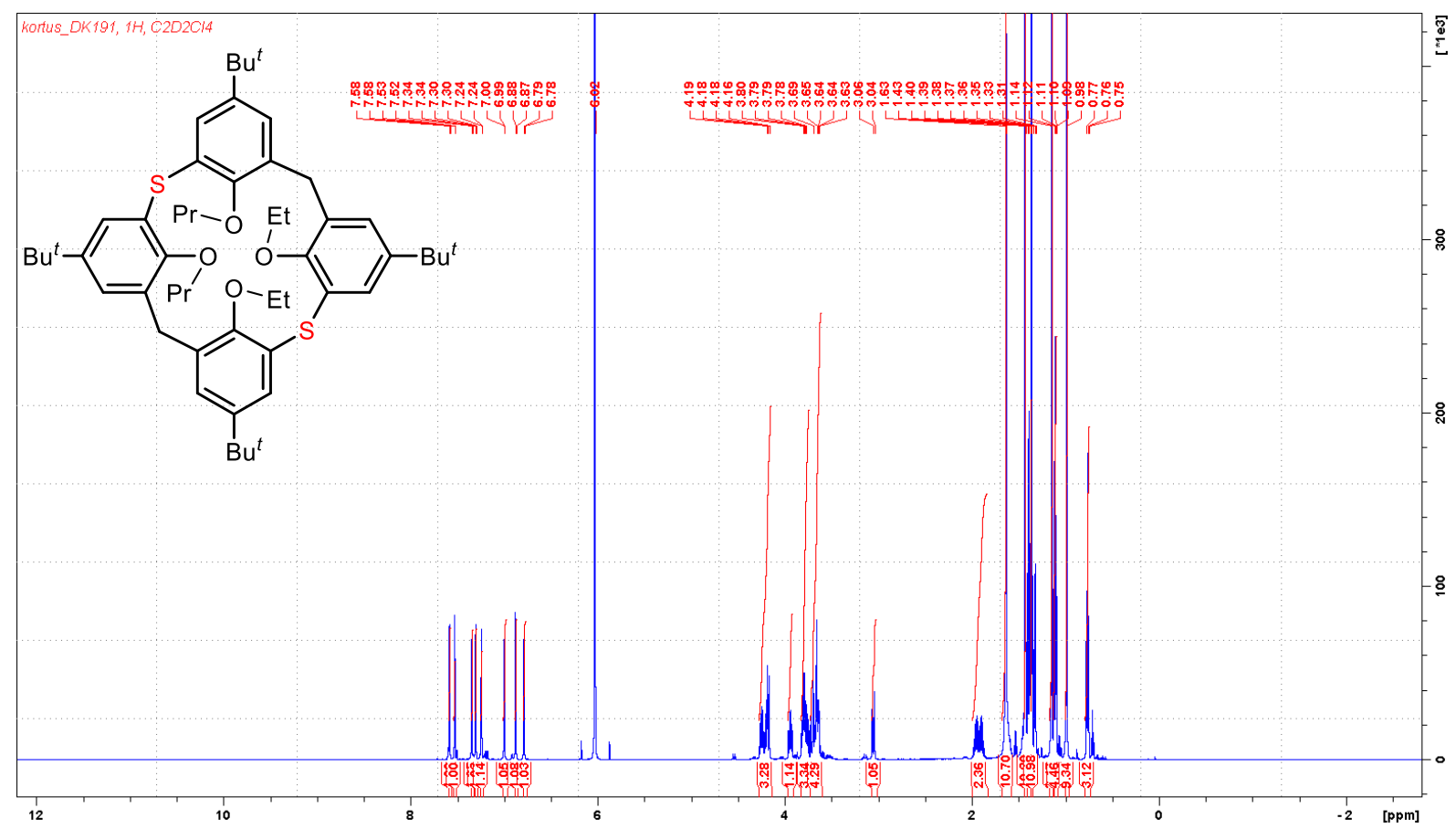

Figure S157. ${ }^{1} \mathrm{H} \mathrm{NMR}$ of compound $16\left(\mathrm{CD}_{2} \mathrm{Cl}_{2}, 600 \mathrm{MHz}\right)$.

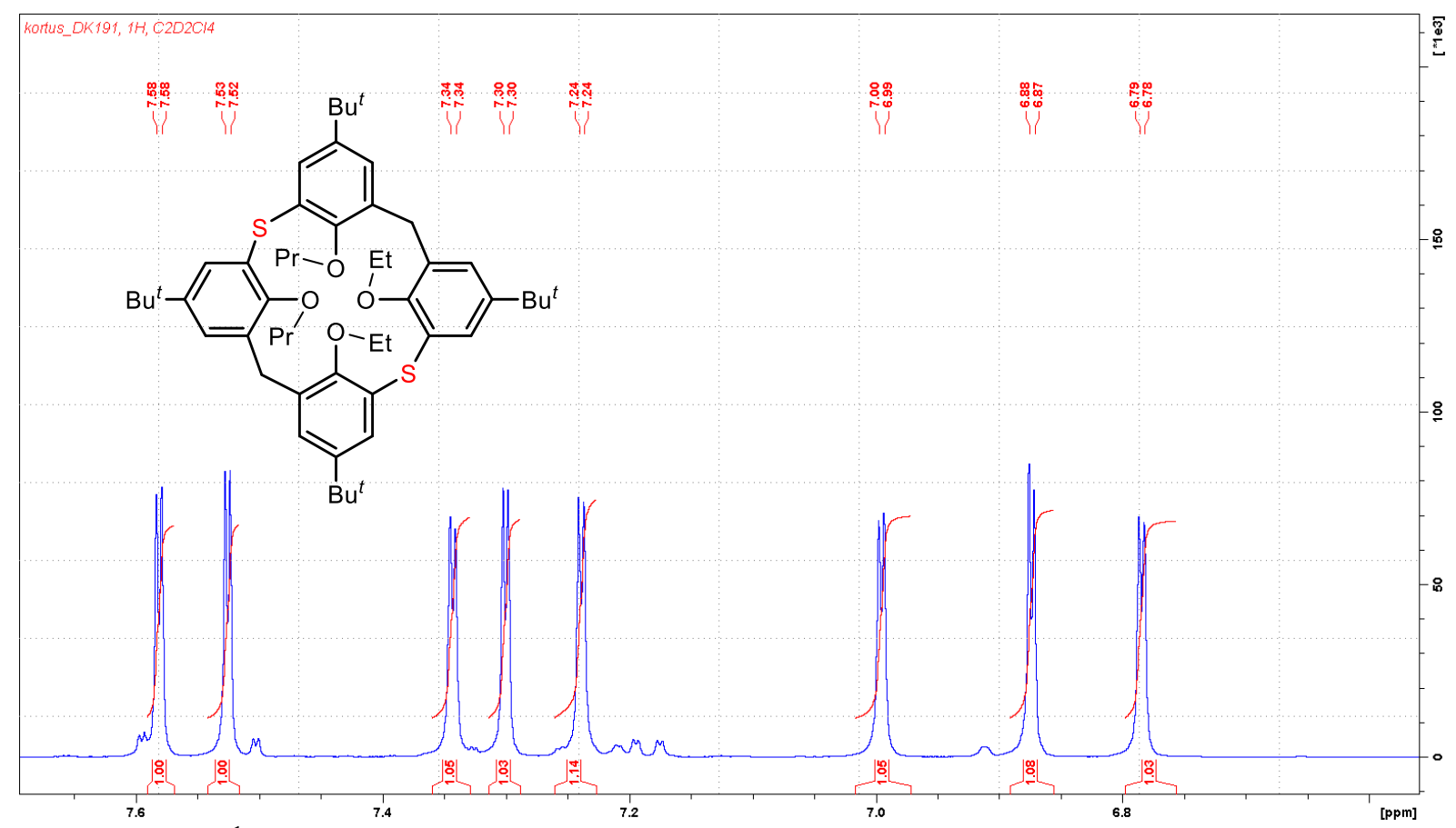

Figure S158. ${ }^{1} \mathrm{H}$ NMR of compound 16, aromatic region $\left(\mathrm{CD}_{2} \mathrm{Cl}_{2}, 600 \mathrm{MHz}\right)$. 


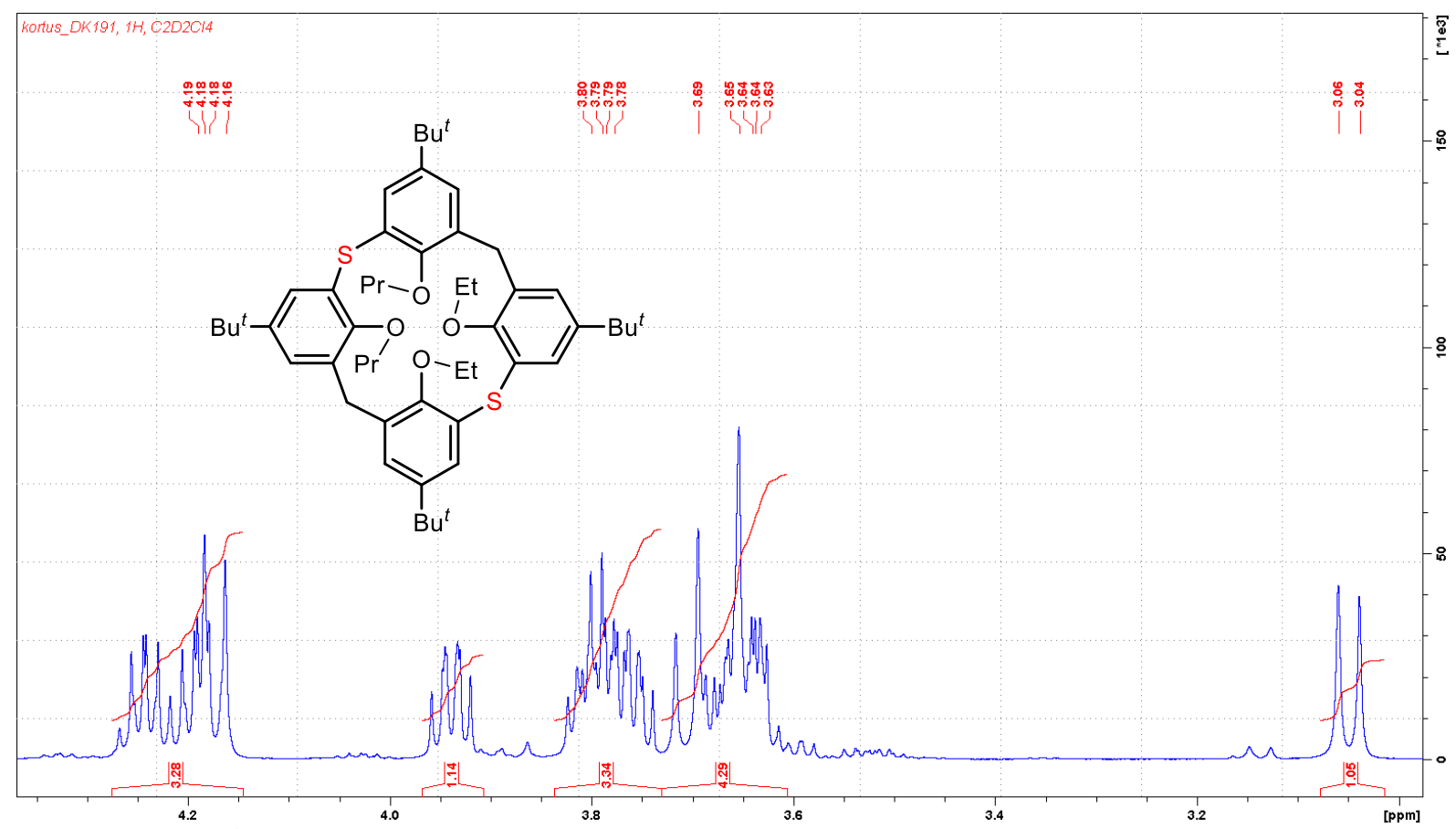

Figure S159. ${ }^{1} \mathrm{H}$ NMR of compound 16, bridge region $\left(\mathrm{CD}_{2} \mathrm{Cl}_{2}, 600 \mathrm{MHz}\right)$.

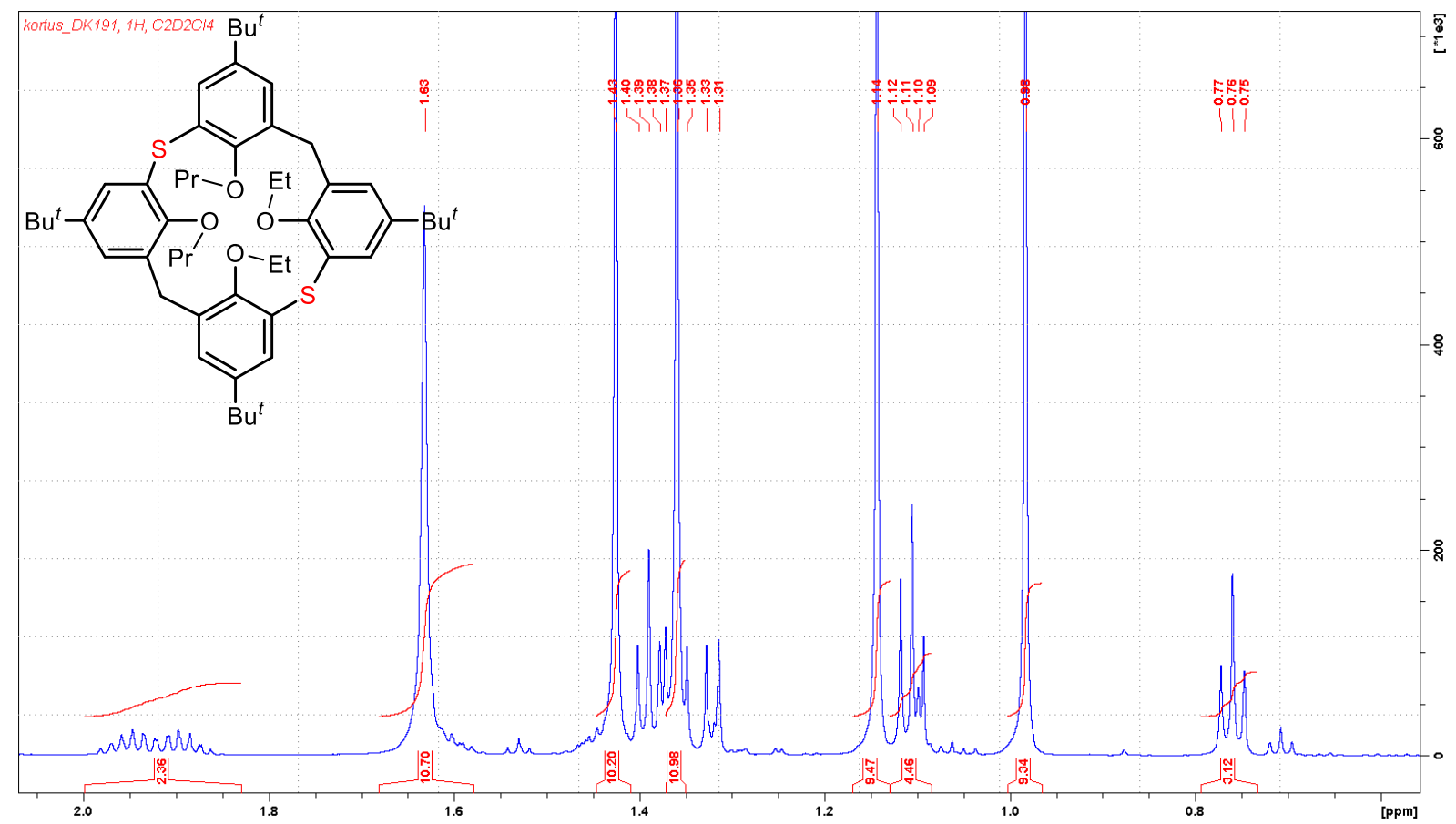

Figure S160. ${ }^{1} \mathrm{H} \mathrm{NMR}$ of compound 16, aliphatic region $\left(\mathrm{CD}_{2} \mathrm{Cl}_{2}, 600 \mathrm{MHz}\right)$. 


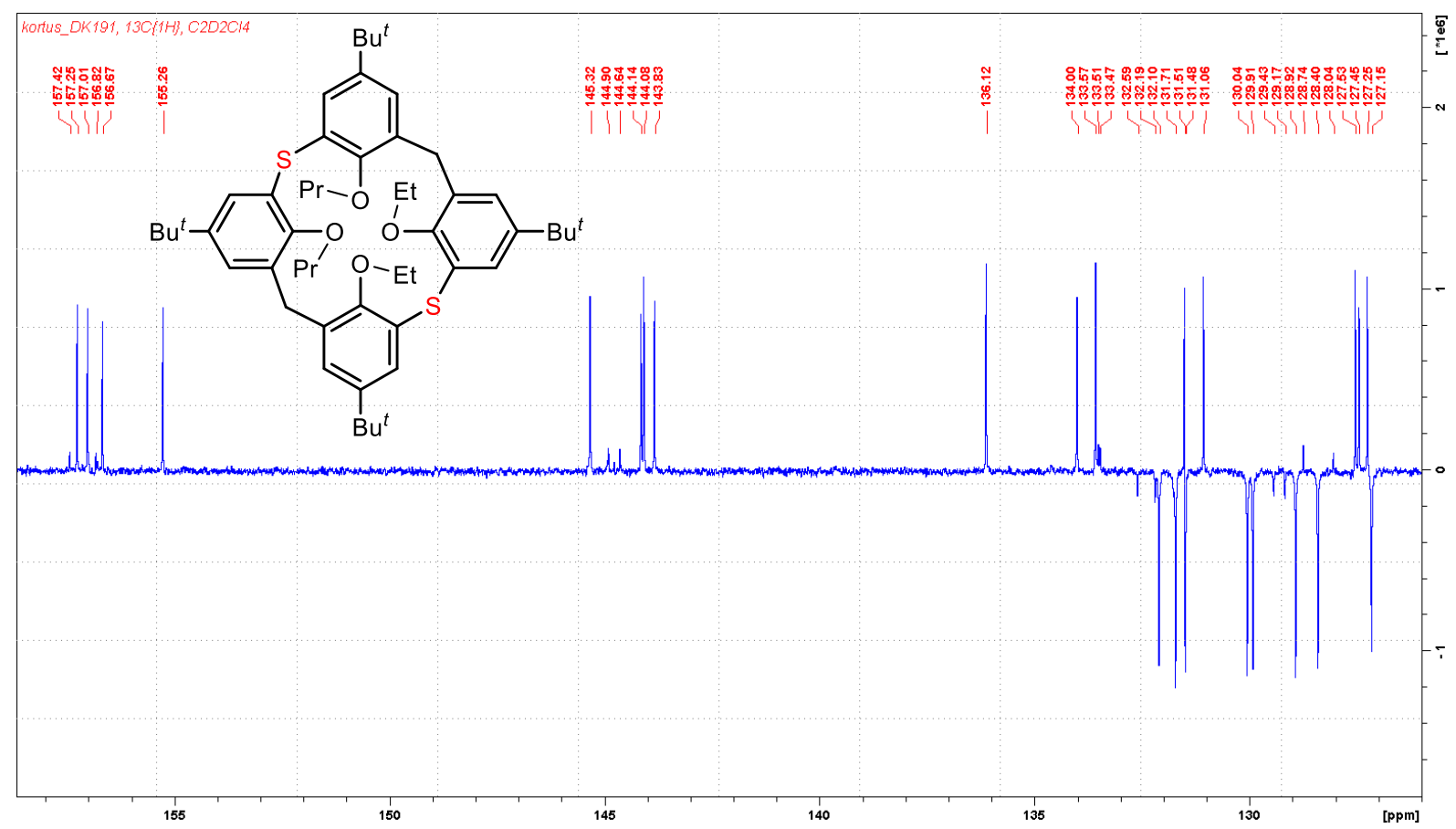

Figure S161. ${ }^{13} \mathrm{C}\left\{{ }^{1} \mathrm{H}\right\}$ NMR of compound $16\left(\mathrm{CD}_{2} \mathrm{Cl}_{2}, 151 \mathrm{MHz}\right)$.

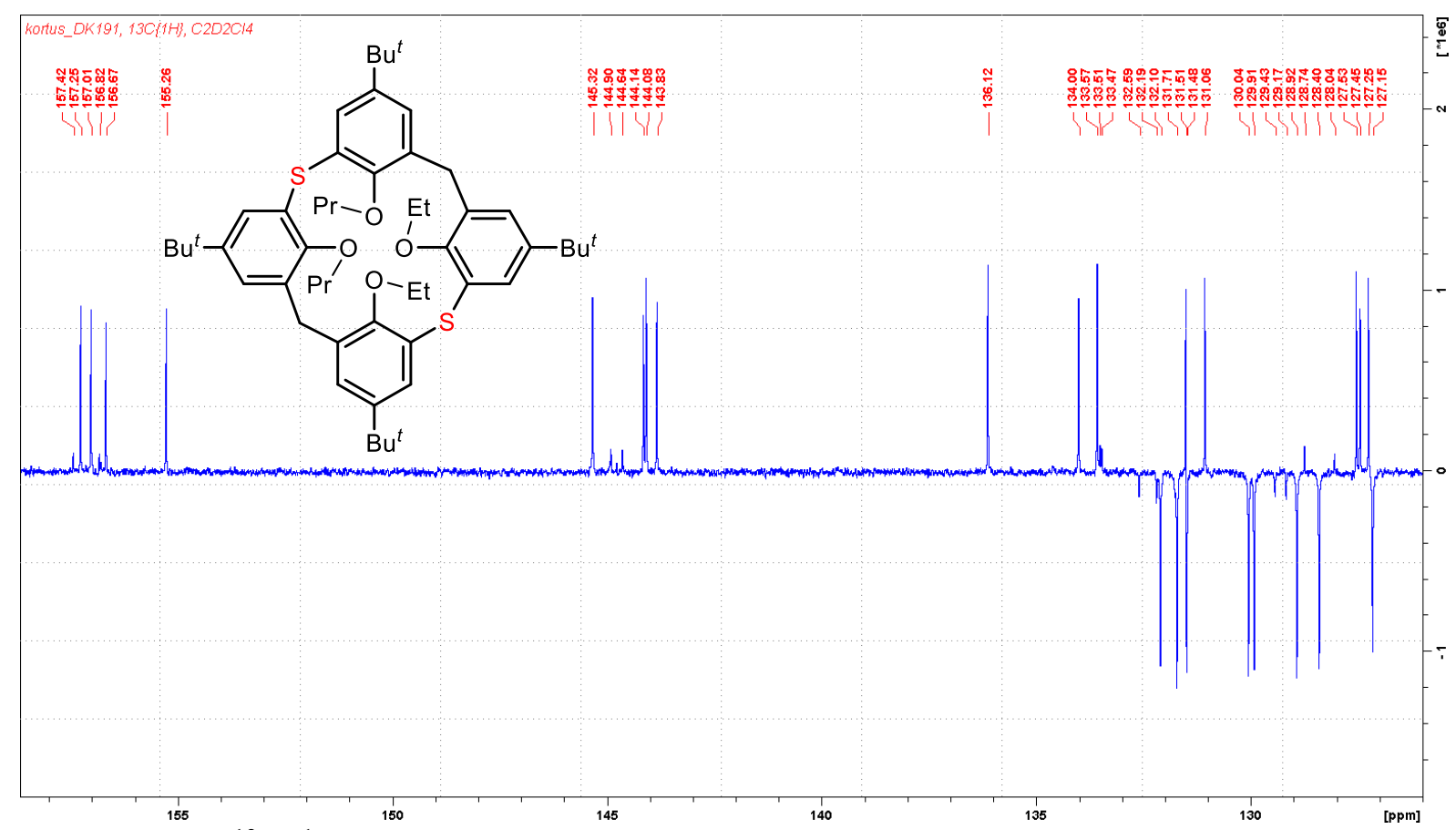

Figure S162. ${ }^{13} \mathrm{C}\left\{{ }^{1} \mathrm{H}\right\}$ NMR of compound 16, aromatic region $\left(\mathrm{CD}_{2} \mathrm{Cl}_{2}, 151 \mathrm{MHz}\right)$. 


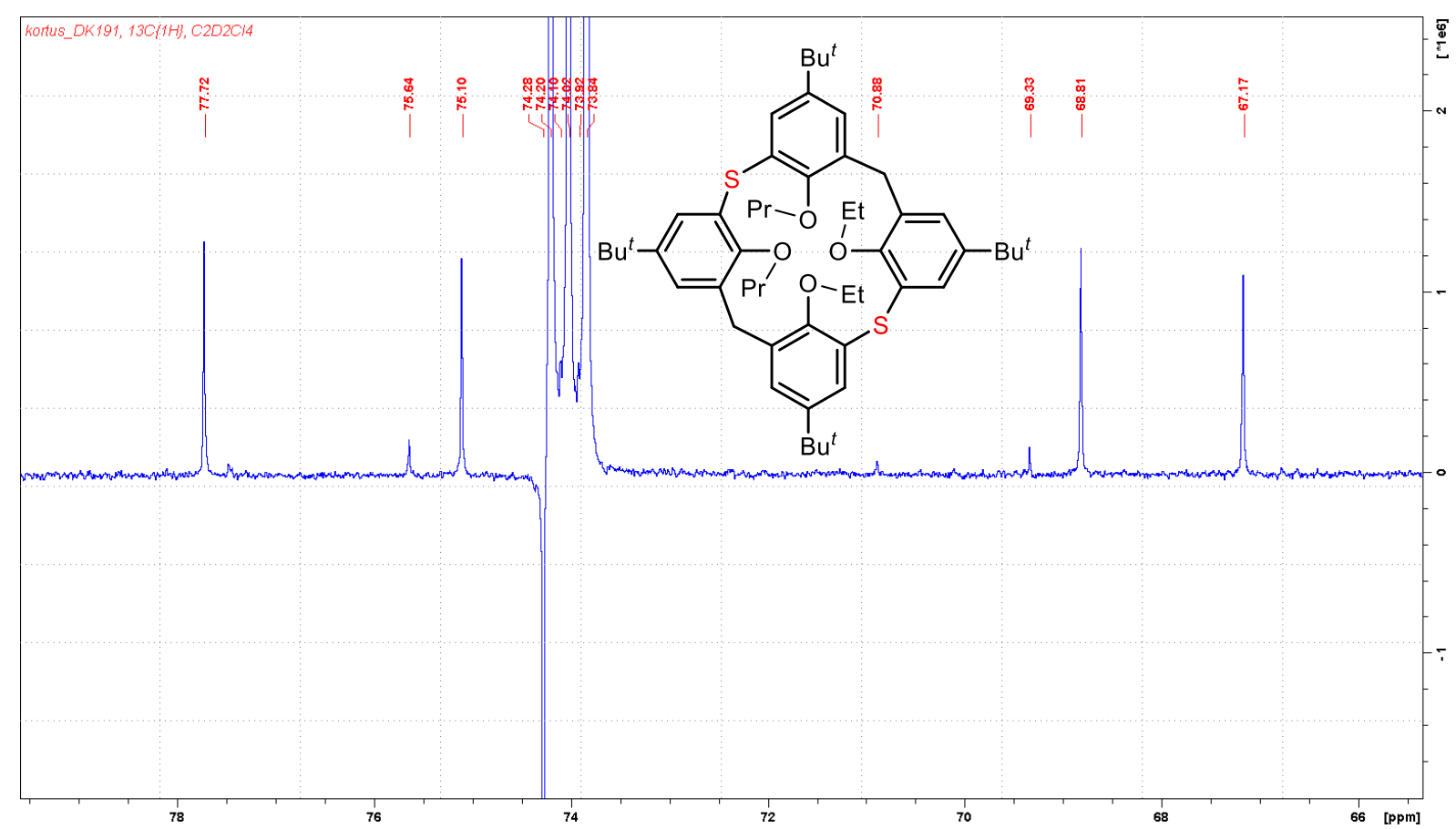

Figure S163. ${ }^{13} \mathrm{C}\left\{{ }^{1} \mathrm{H}\right\}$ NMR of compound 16, aliphatic region $\left(\mathrm{CD}_{2} \mathrm{Cl}_{2}, 151 \mathrm{MHz}\right)$.

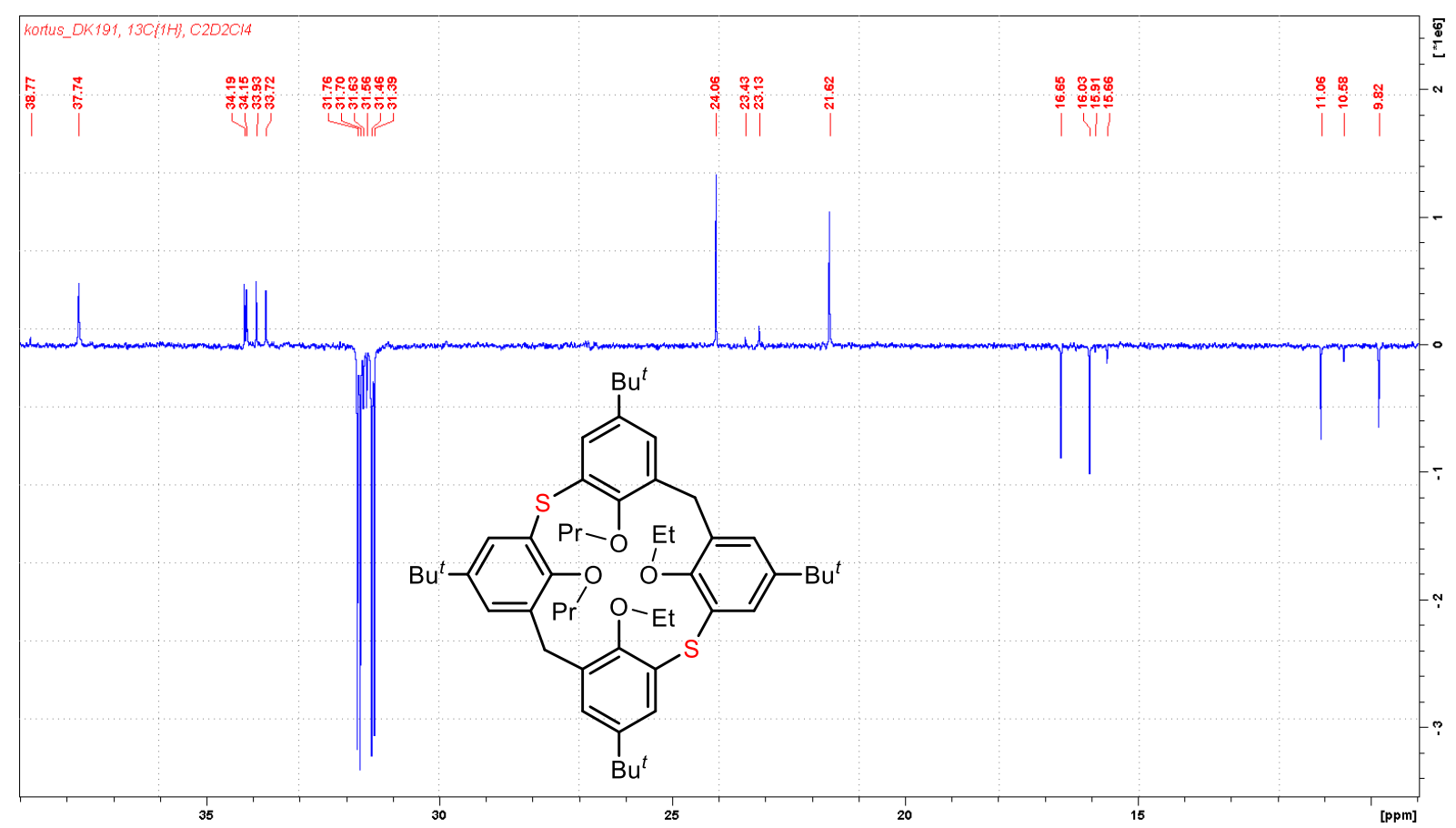

Figure S164. ${ }^{13} \mathrm{C}\left\{{ }^{1} \mathrm{H}\right\}$ NMR of compound 16, aliphatic region $\left(\mathrm{CD}_{2} \mathrm{Cl}_{2}, 151 \mathrm{MHz}\right)$. 


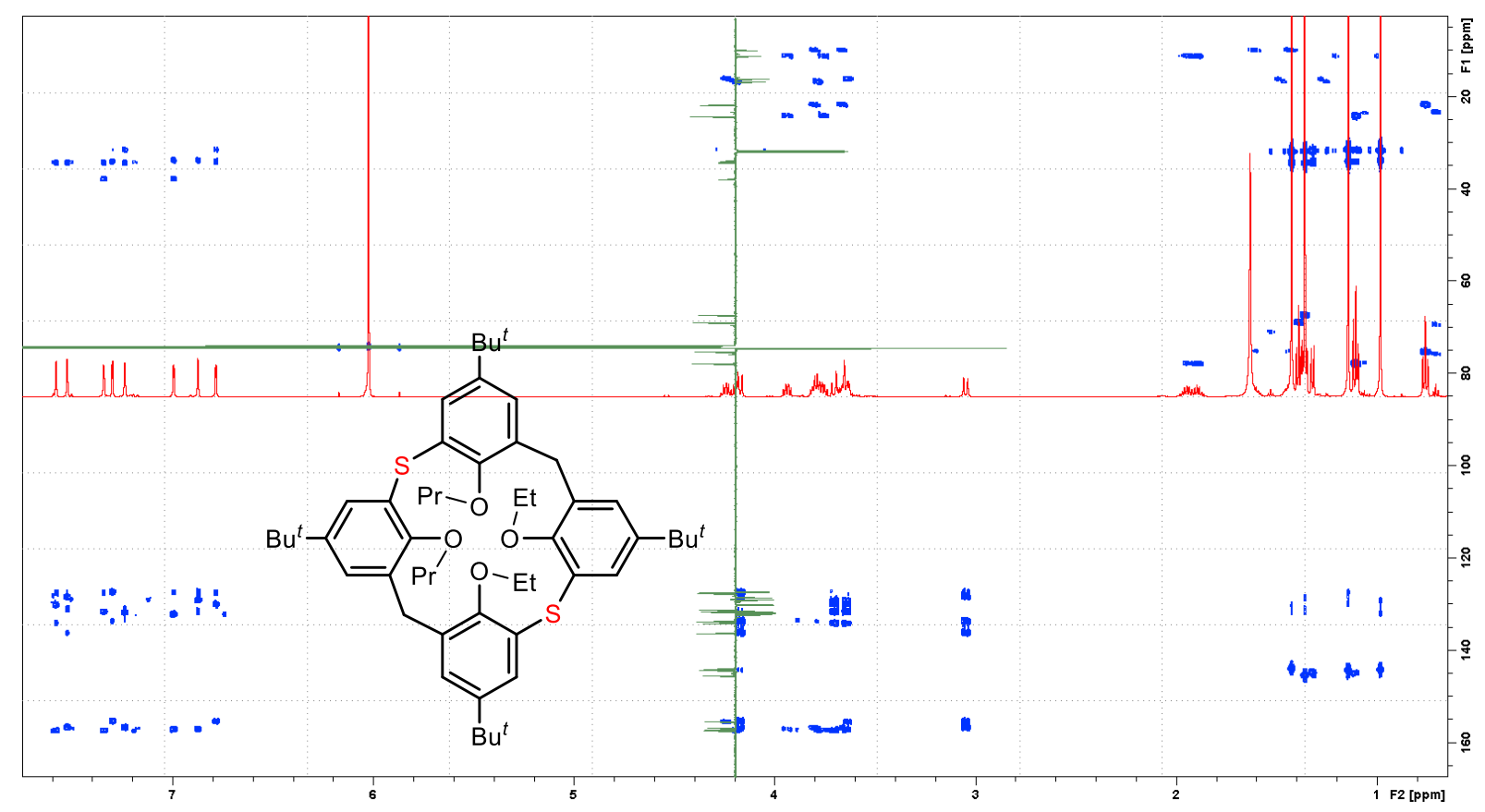

Figure S165. HMBC NMR of compound $16\left(\mathrm{CD}_{2} \mathrm{Cl}_{2}\right)$.

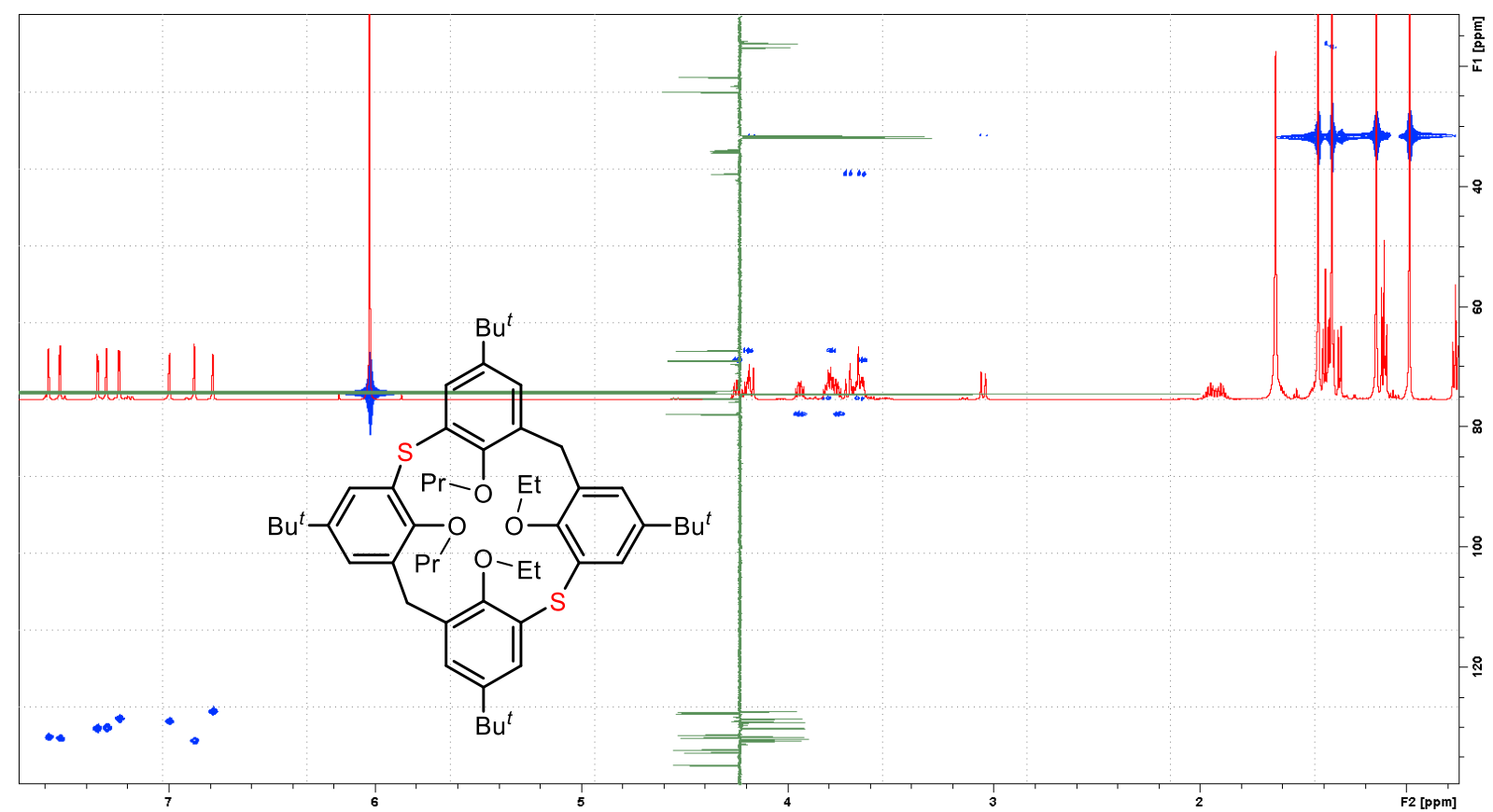

Figure S166. HMQC NMR of compound $16\left(\mathrm{CD}_{2} \mathrm{Cl}_{2}\right)$. 


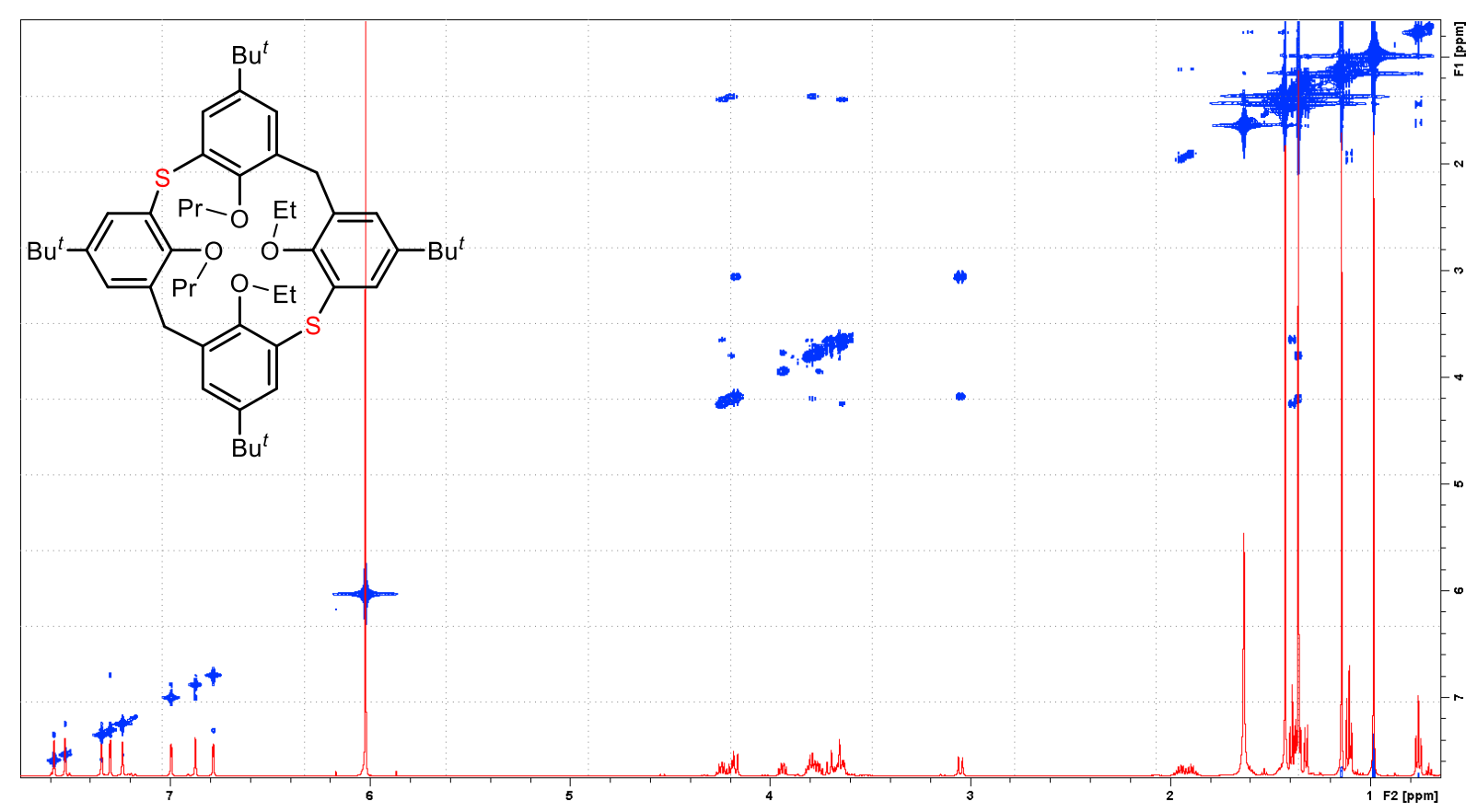

Figure S167. COSY NMR of compound $16\left(\mathrm{CD}_{2} \mathrm{Cl}_{2}\right)$.

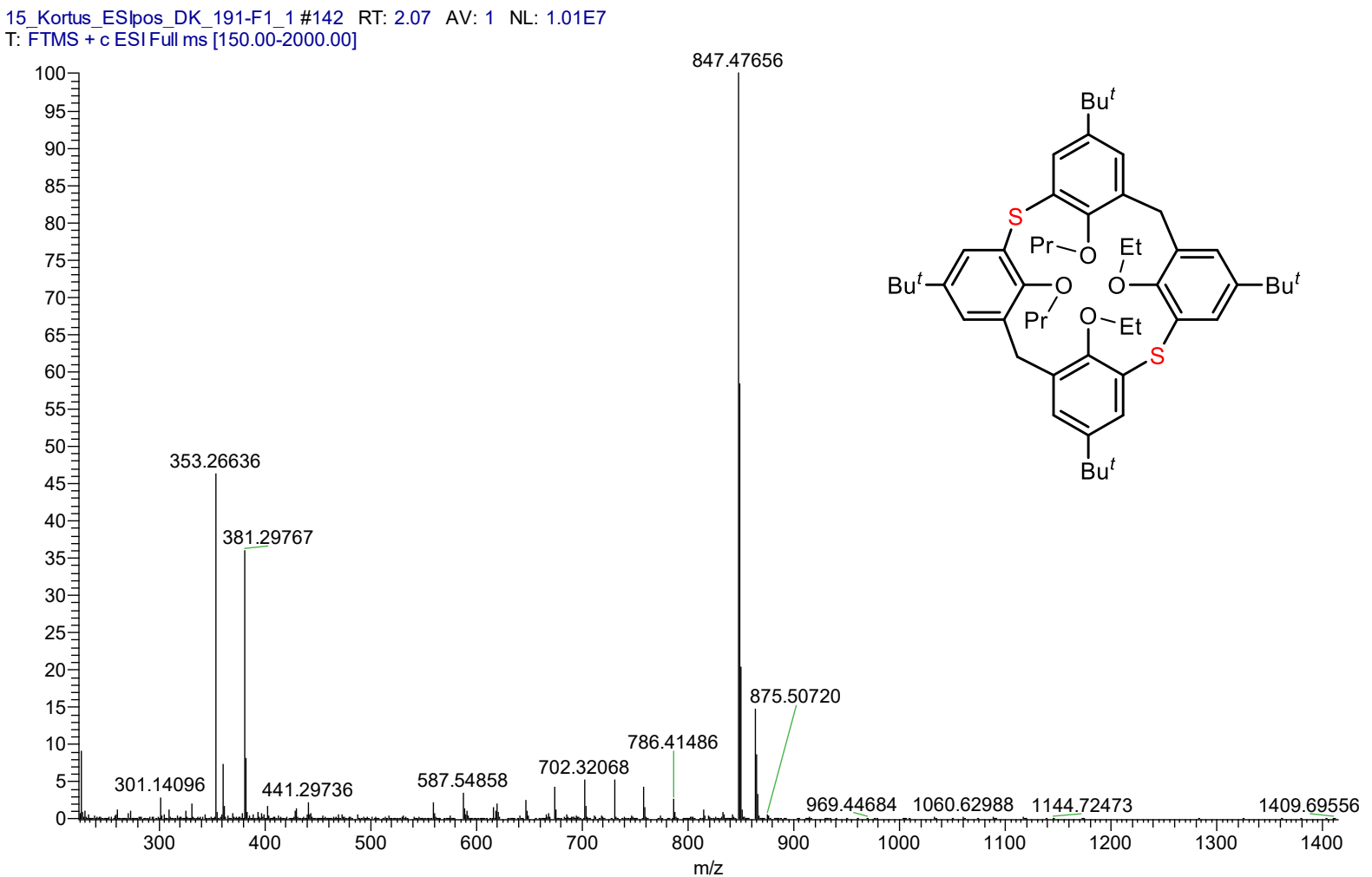

Figure S168. HRMS of compound $16\left(\mathrm{ESI}^{+}\right)$. 


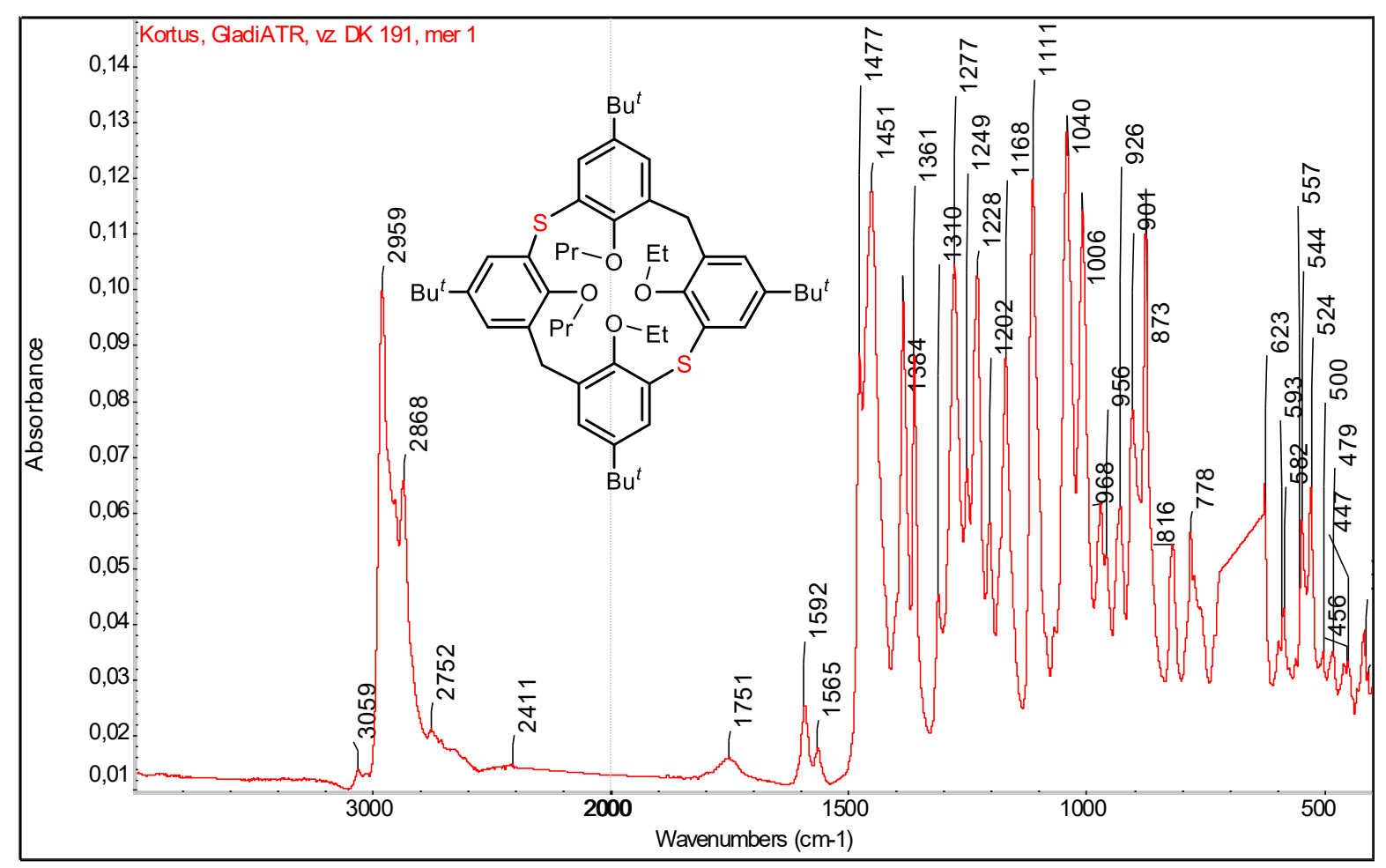

Figure S169. IR of compound 16 (ATR). 


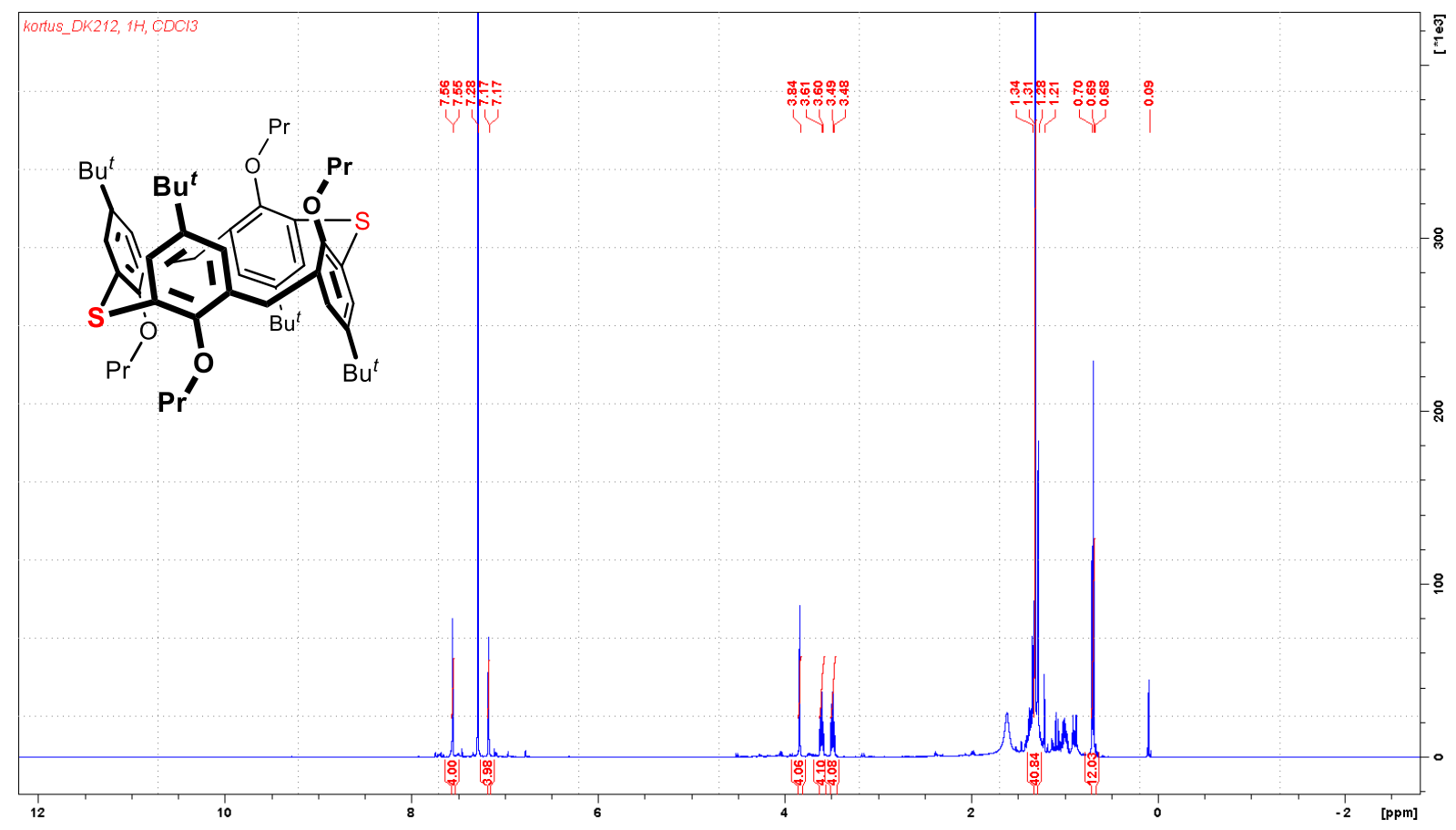

Figure S170. ${ }^{1} \mathrm{H}$ NMR of compound $17\left(\mathrm{CDCl}_{3}, 600 \mathrm{MHz}\right)$.

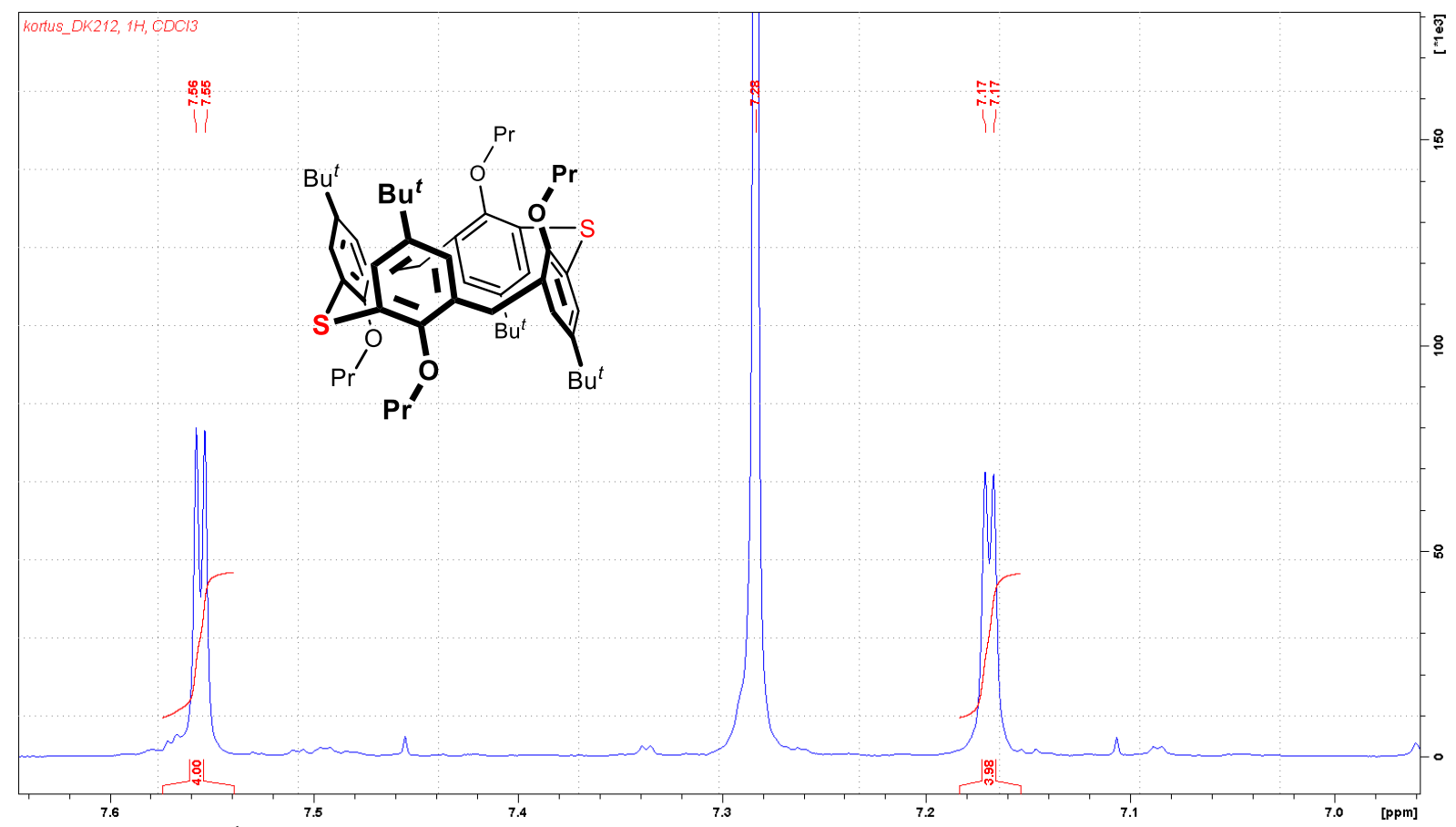

Figure S171. ${ }^{1} \mathrm{H}$ NMR of compound 17 , aromatic region $\left(\mathrm{CDCl}_{3}, 600 \mathrm{MHz}\right)$. 


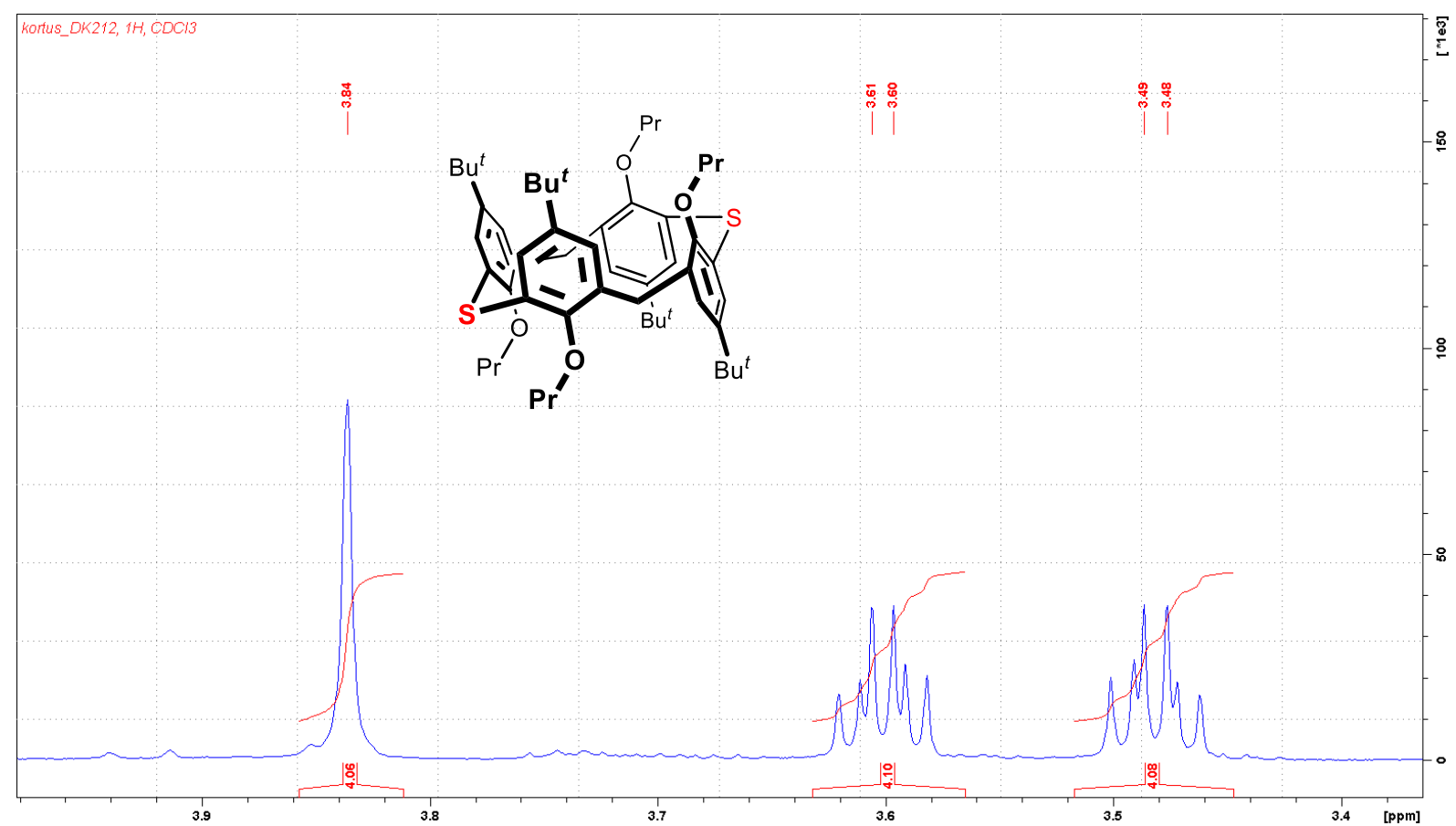

Figure S172. ${ }^{1} \mathrm{H} \mathrm{NMR}$ of compound 17, bridge region $\left(\mathrm{CDCl}_{3}, 600 \mathrm{MHz}\right)$.

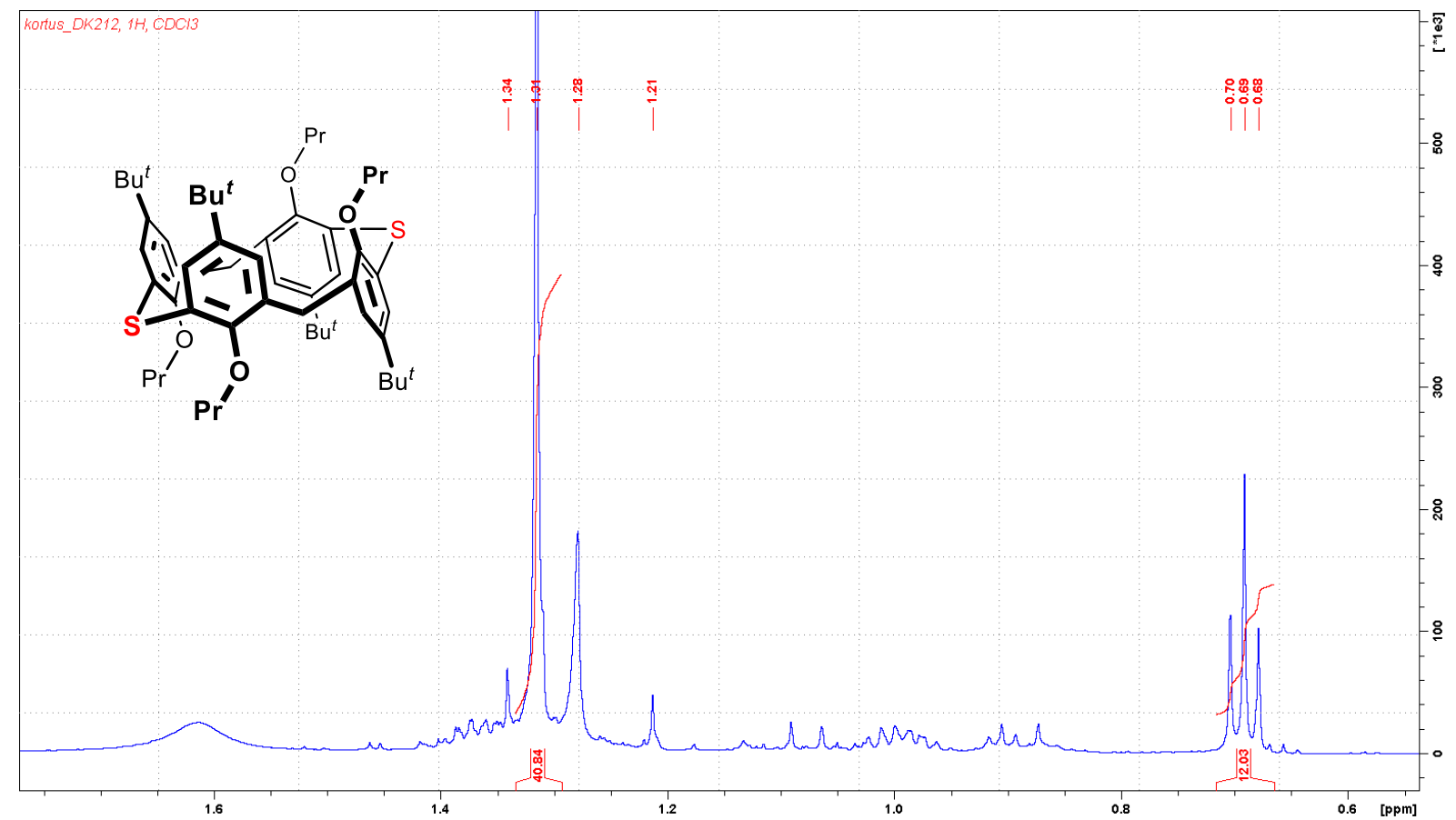

Figure S173. ${ }^{1} \mathrm{H}$ NMR of compound 17, aliphatic region $\left(\mathrm{CDCl}_{3}, 600 \mathrm{MHz}\right)$. 


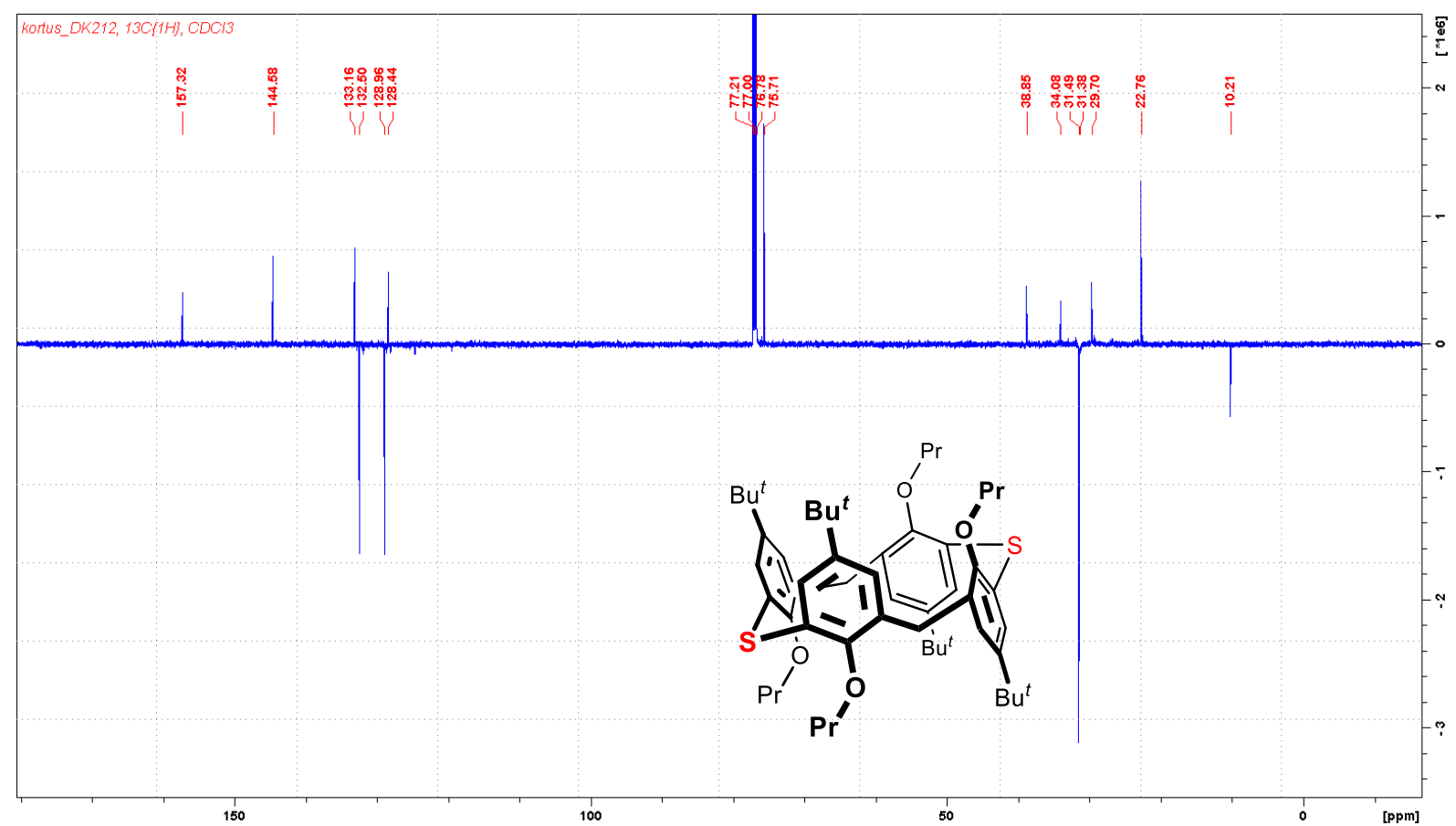

Figure S174. ${ }^{13} \mathrm{C}\left\{{ }^{1} \mathrm{H}\right\}$ NMR of compound $17\left(\mathrm{CDCl}_{3}, 151 \mathrm{MHz}\right)$.

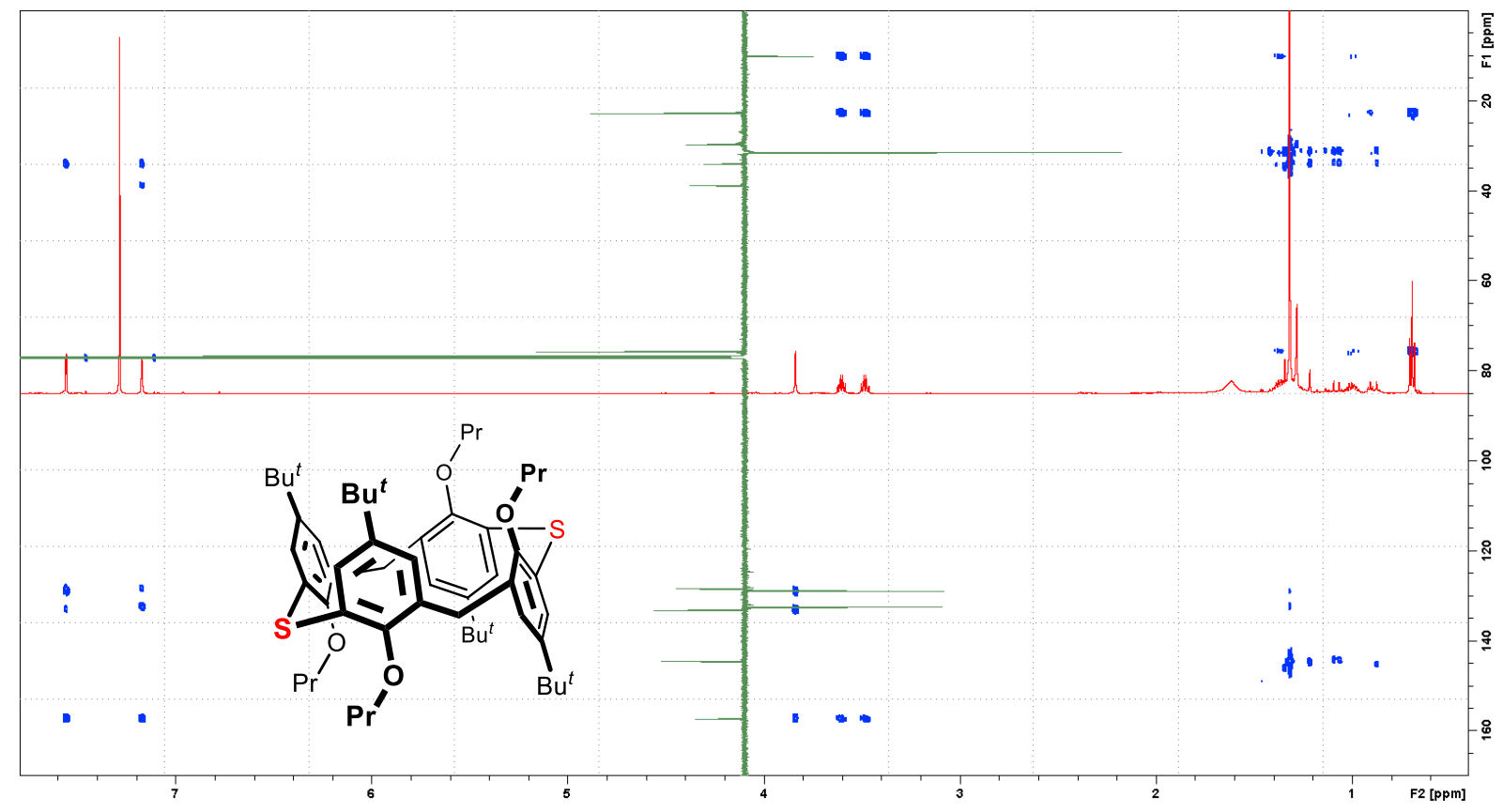

Figure S175. HMBC NMR of compound $17\left(\mathrm{CDCl}_{3}\right)$. 


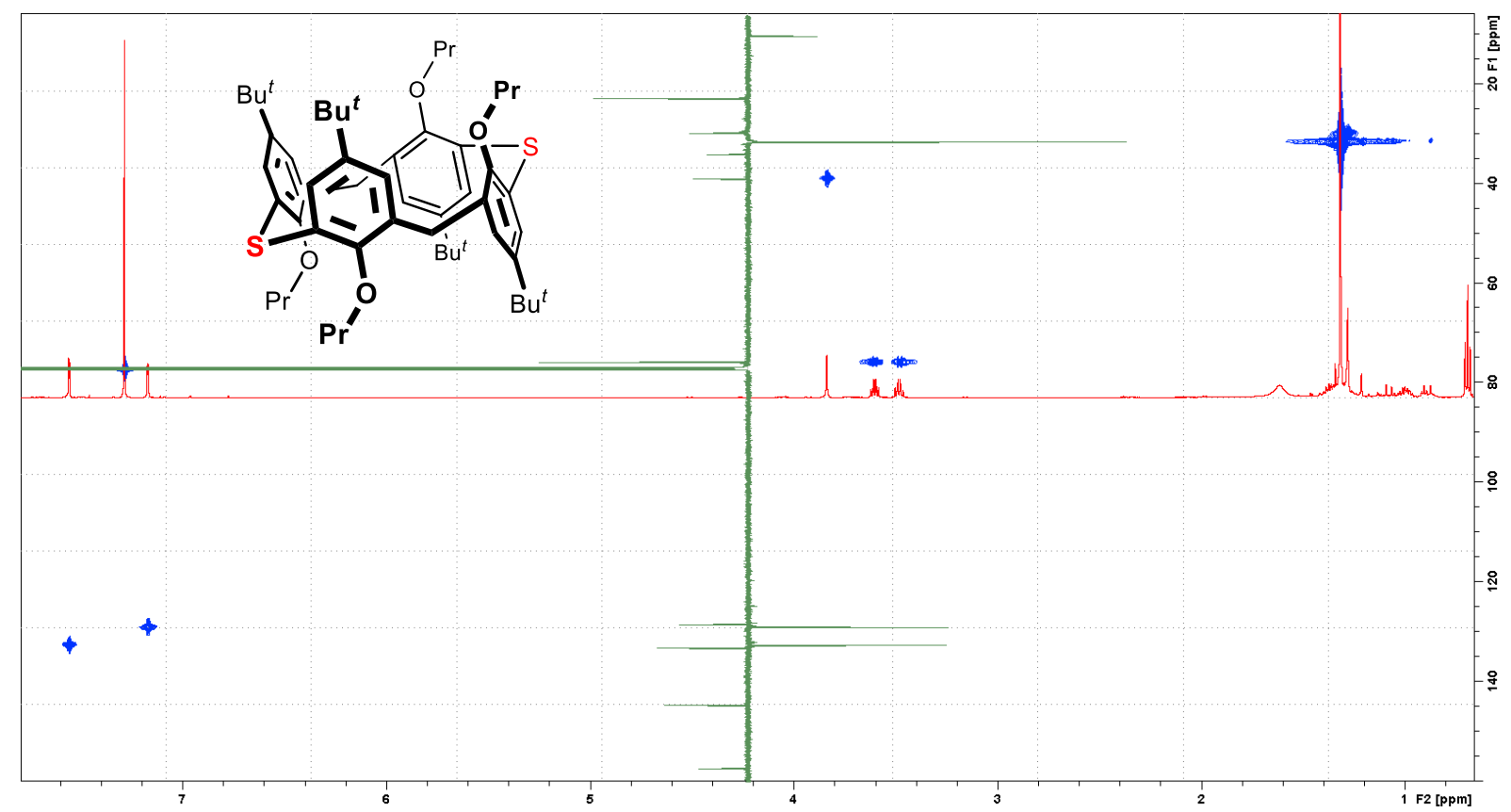

Figure S176. HMQC NMR of compound $17\left(\mathrm{CDCl}_{3}\right)$.

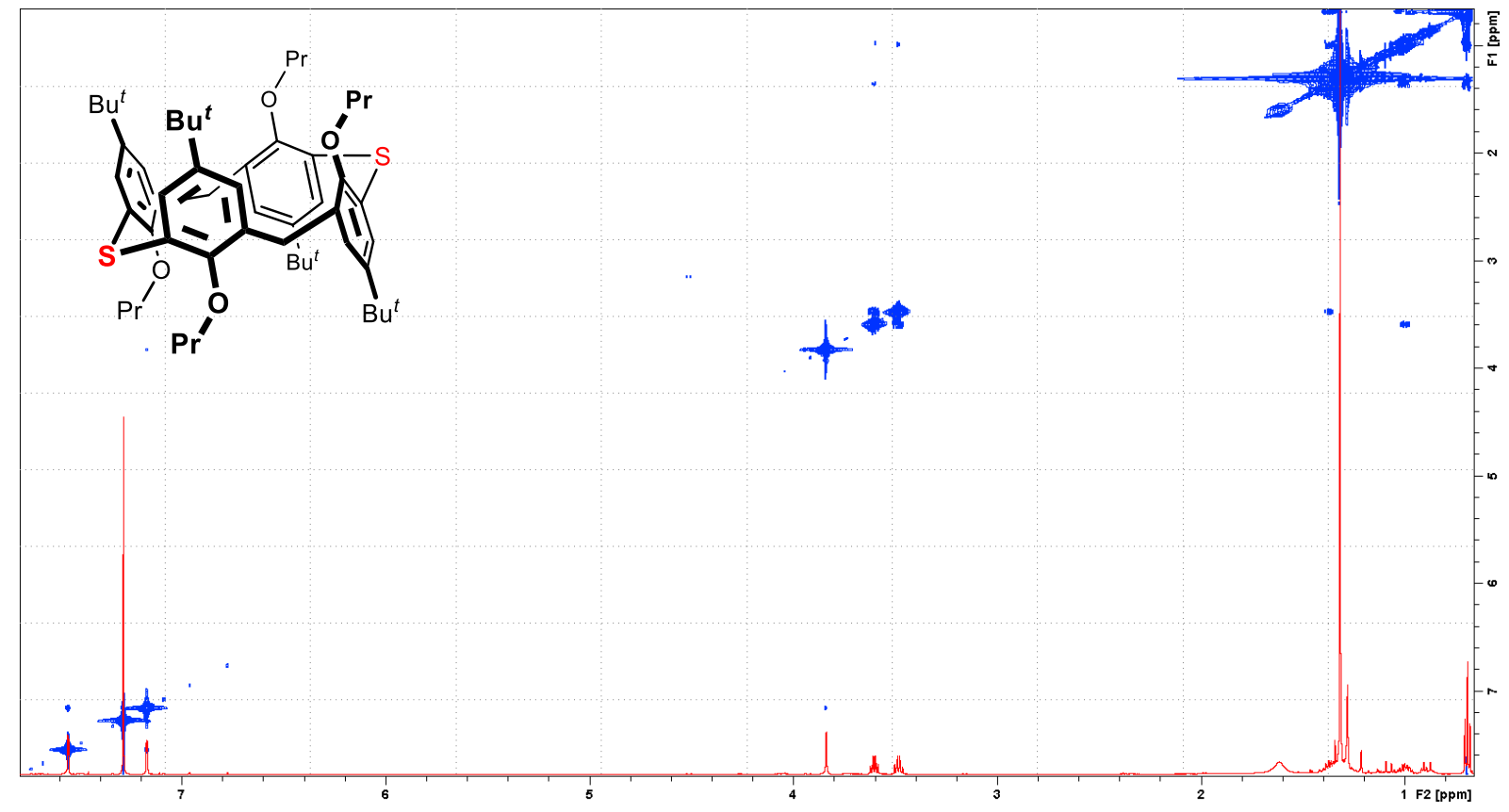

Figure S177. COSY NMR of compound $17\left(\mathrm{CDCl}_{3}\right)$. 


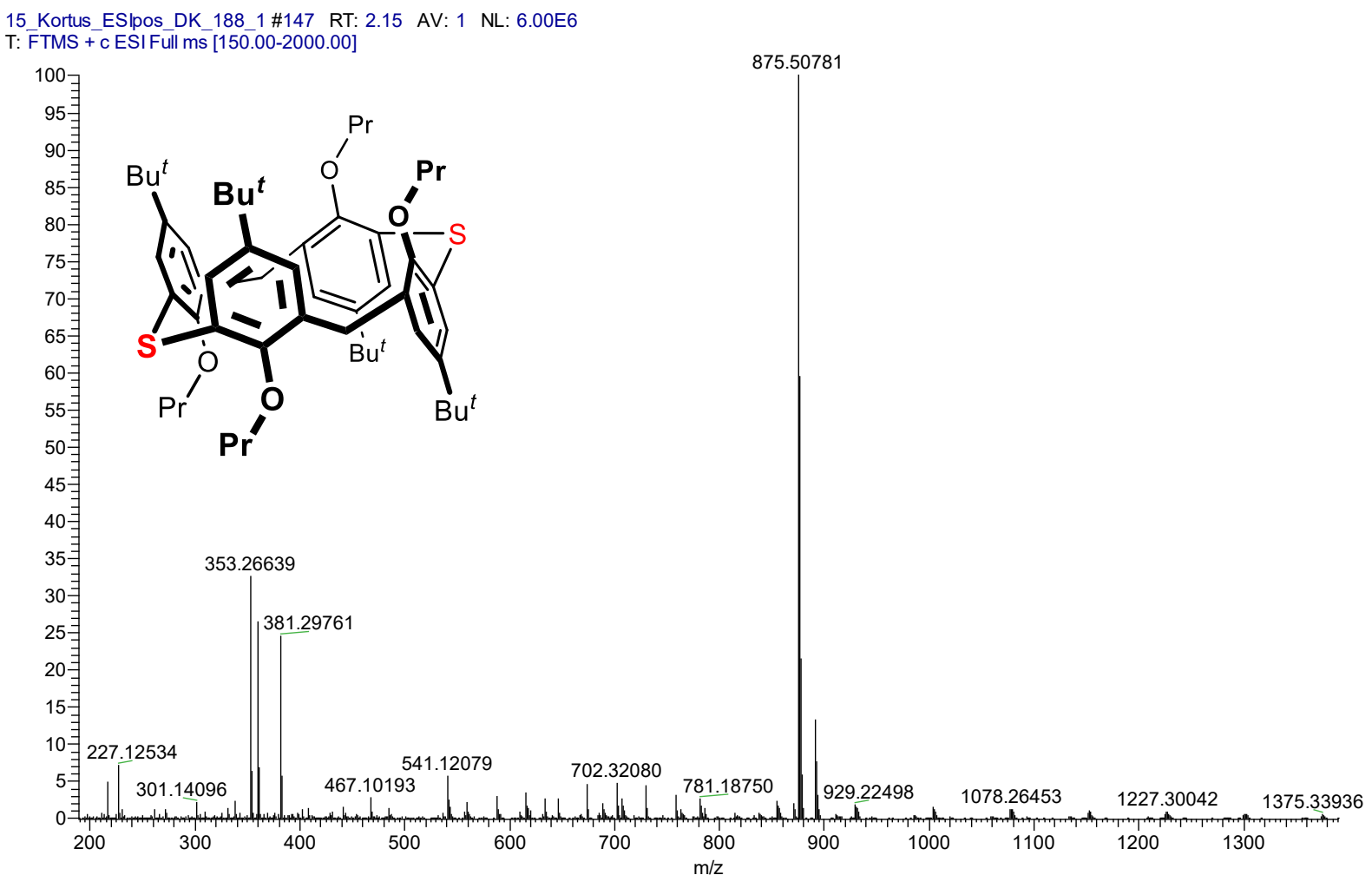

Figure S178. HRMS of compound $17\left(\mathrm{ESI}^{+}\right)$.

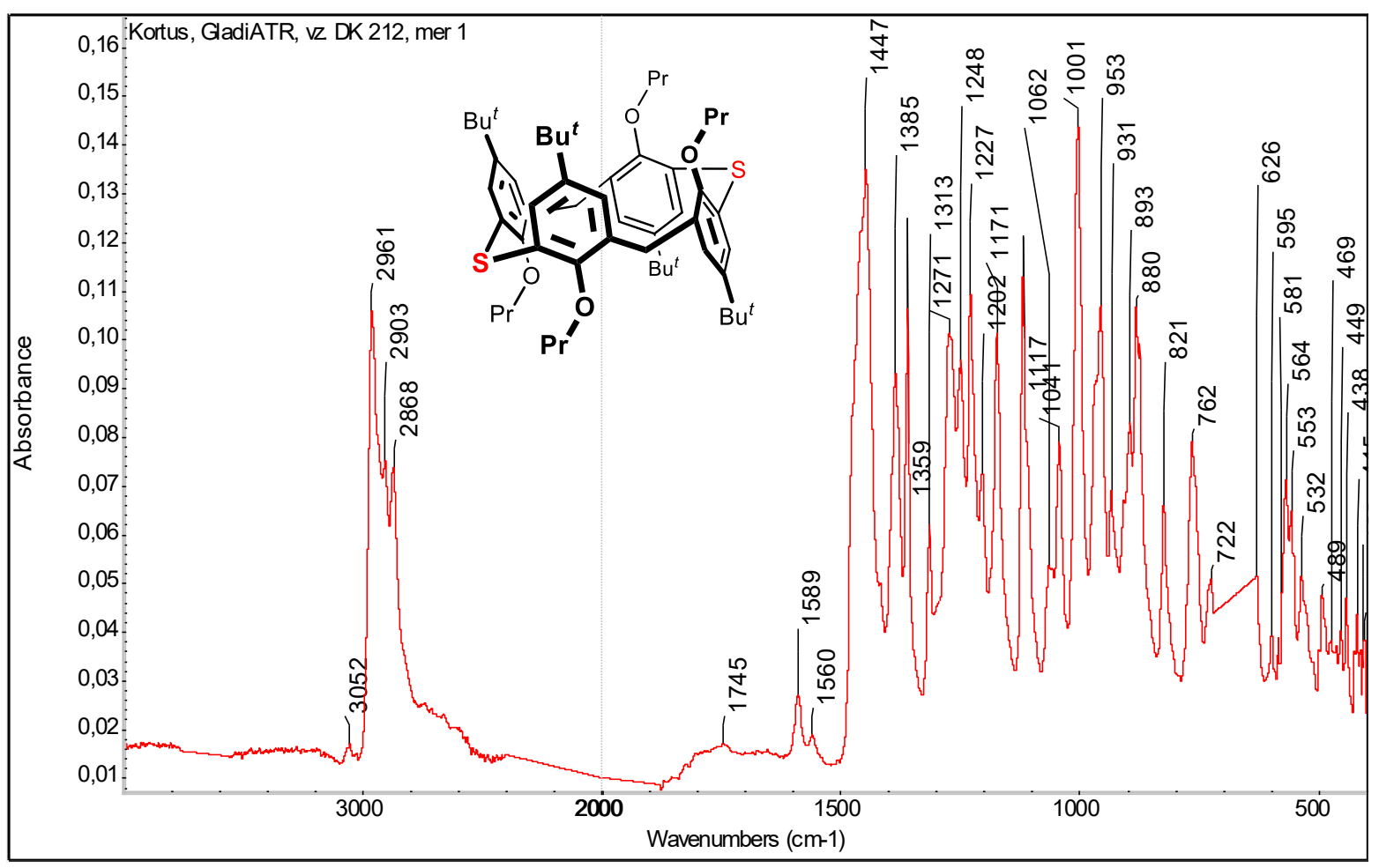

Figure S179. IR of compound 17 (ATR) 


\section{NOE MEASUREMENTS}

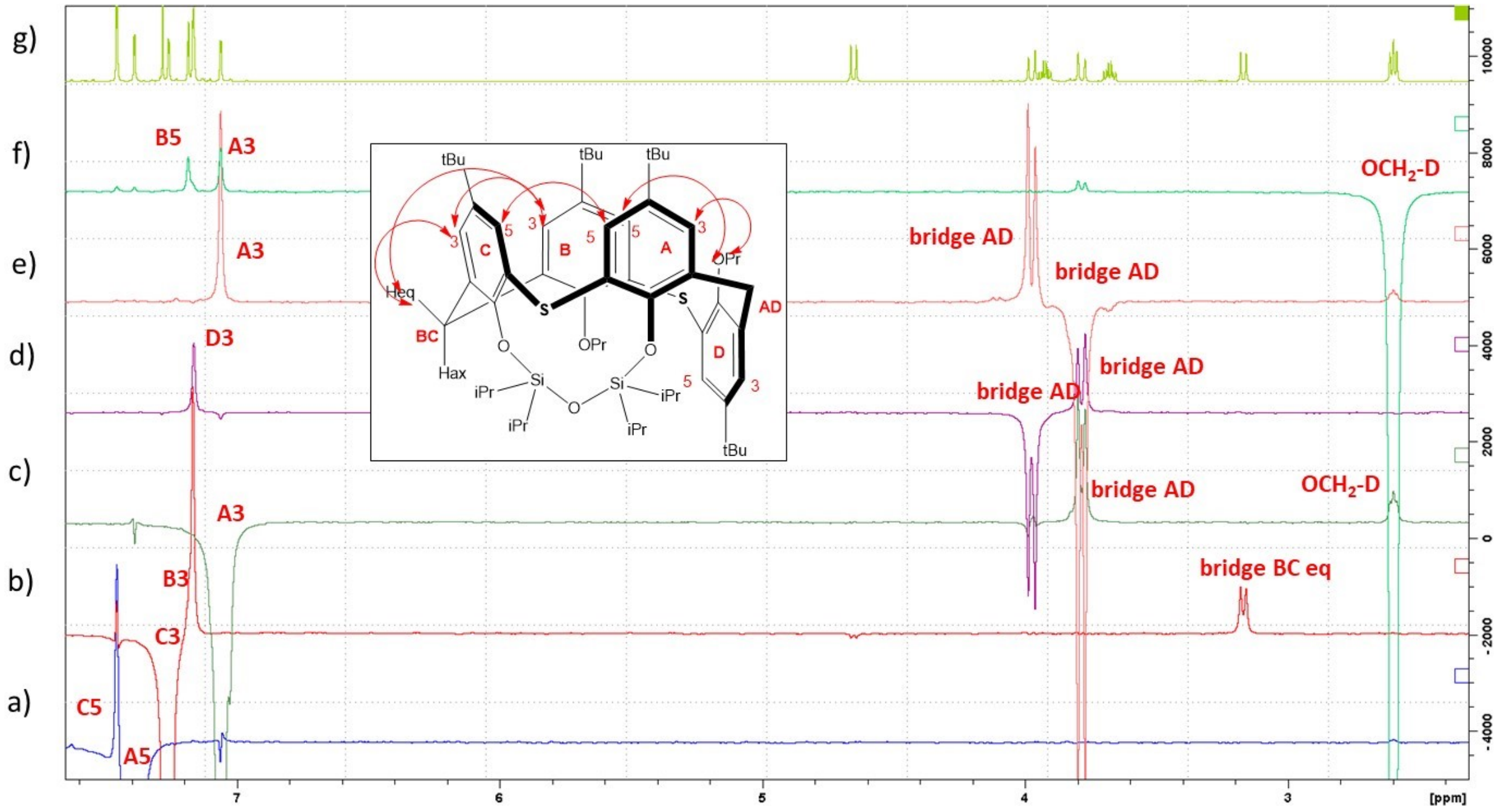

Figure S180. NOE spectra of compound 4 (600 MHz, $\left.\mathrm{CD}_{2} \mathrm{Cl}_{2}, 298 \mathrm{~K}\right)$ : a) A5 irradiated, b) C3 irradiated, c) A3 irradiated, d) bridge AD irradiated, e) bridge $\mathrm{AD}$ irradiated, f) $\mathrm{OCH}_{2} \mathrm{D}$ irradiated, g) ${ }^{1} \mathrm{H}$ NMR of compound 4. 


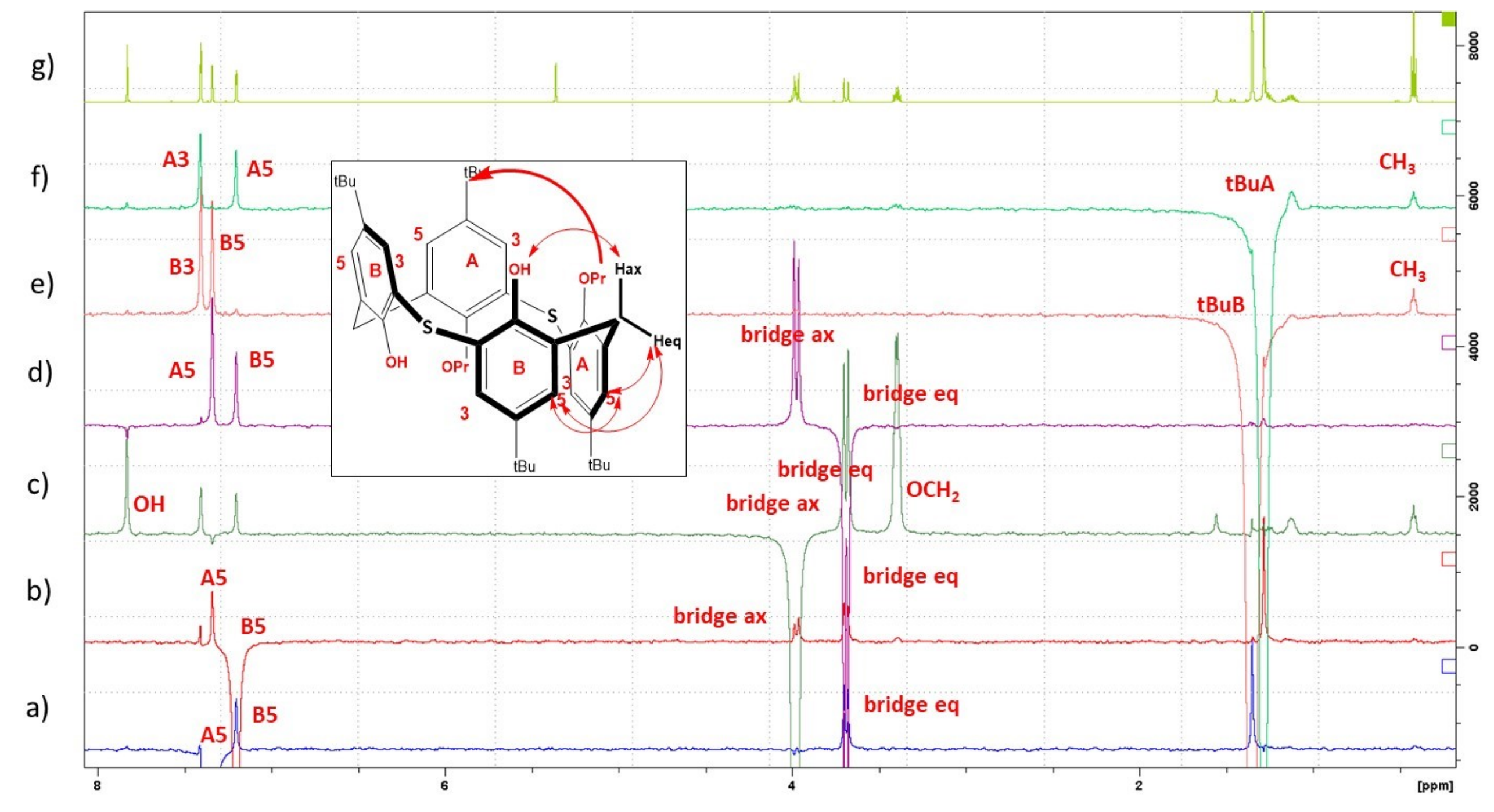

Figure S181. NOE spectra of Compound 7 (600 MHz, $\left.\mathrm{CD}_{2} \mathrm{Cl}_{2}, 298 \mathrm{~K}\right)$ : a) A5 irradiated, b) B5 irradiated, c) bridge ax irradiated, d) bridge eq irradiated, e) $t \mathrm{Bu}-\mathrm{B}$ irradiated, f) $t \mathrm{Bu}-\mathrm{A}$ irradiated, g) ${ }^{1} \mathrm{H}$ NMR of compound 7. 


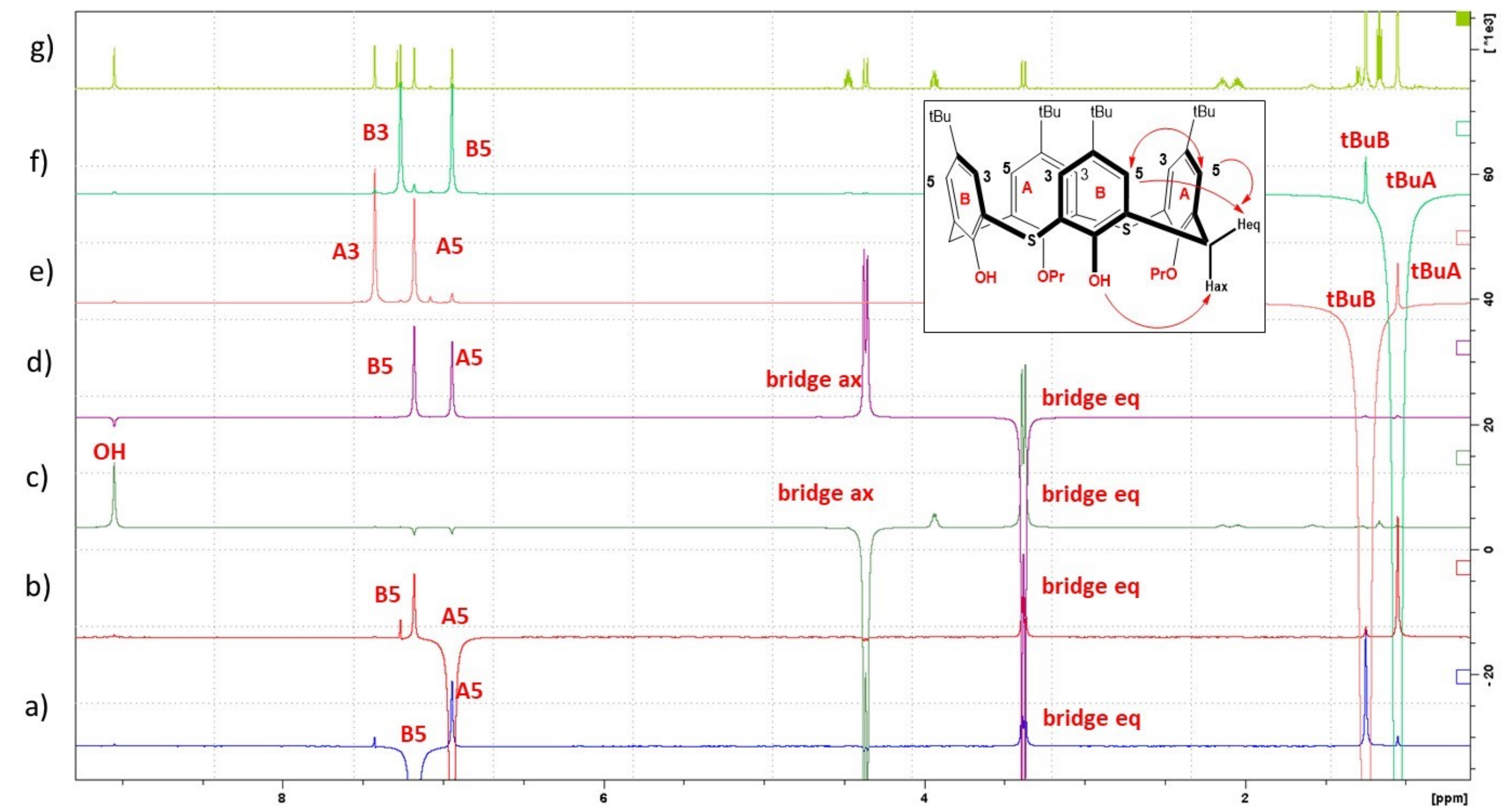

Figure S182. NOE spectra of compound 8 (600 MHz, $\left.\mathrm{CD}_{2} \mathrm{Cl}_{2}, 298 \mathrm{~K}\right)$ : a) B5 irradiated, b) A5 irradiated, c) bridge ax irradiated, d) bridge eq irradiated, e) $t \mathrm{Bu}-\mathrm{B}$ irradiated, f) $t \mathrm{Bu}-\mathrm{A}$ irradiated, g) ${ }^{1} \mathrm{H}$ NMR of compound 8. 


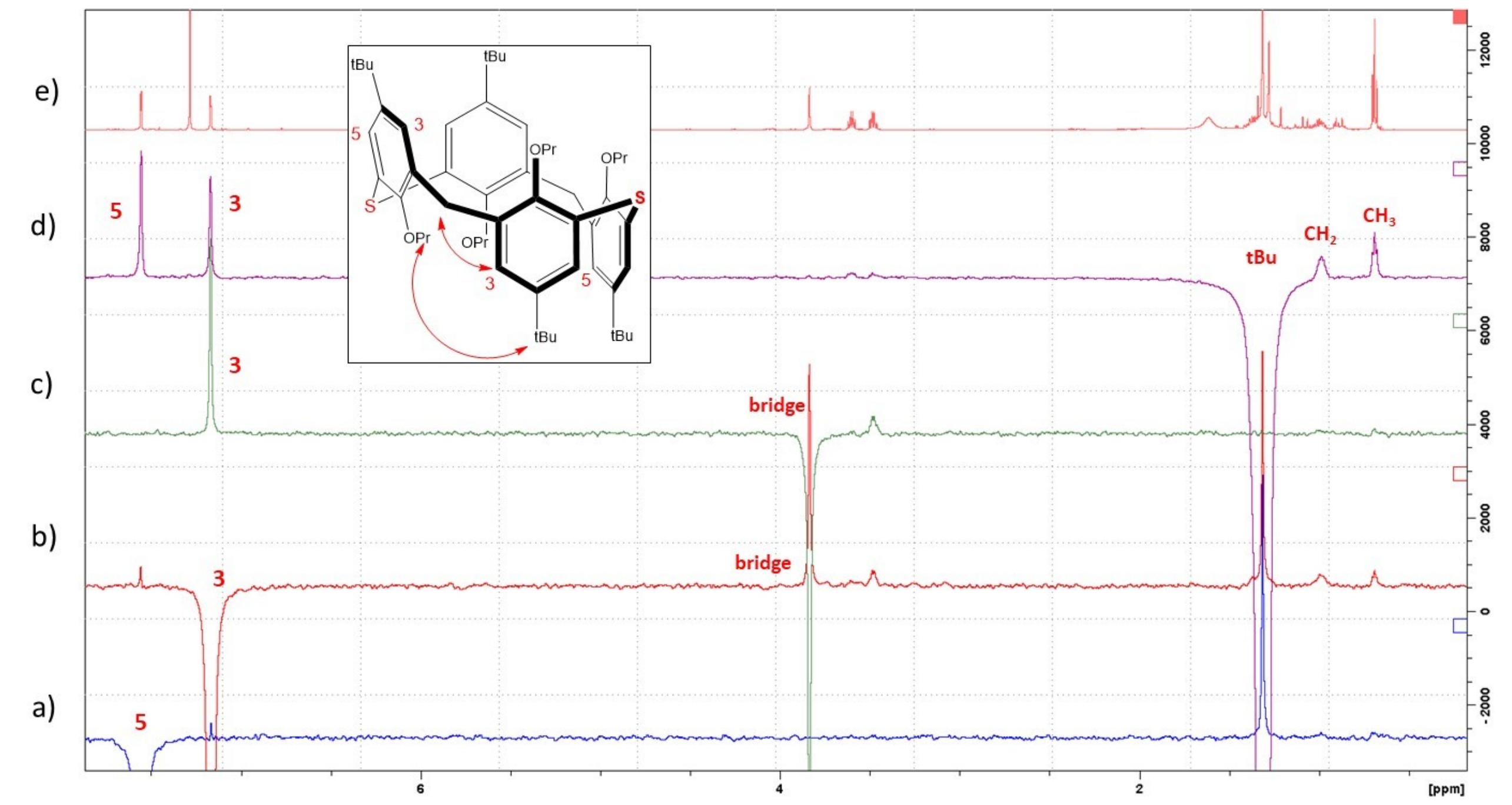

Figure S183. NOE spectra of compound $17\left(600 \mathrm{MHz}, \mathrm{CD}_{2} \mathrm{Cl}_{2}, 298 \mathrm{~K}\right)$ : a) H5 irradiated, b) H3 irradiated, c) bridge irradiated, d) $t \mathrm{Bu}$ irradiated, e) ${ }^{1} \mathrm{H}$ NMR spectrum of compound 17. 


\section{CRYSTALLOGRAPHIC STRUCTURES}

In all cases, slow evaporation of the solvents $\left(\mathrm{CH}_{2} \mathrm{Cl}_{2} / \mathrm{CH}_{3} \mathrm{OH}\right.$ mixtures) was used to crystallize the products from their nearly saturated solutions at room temperature.

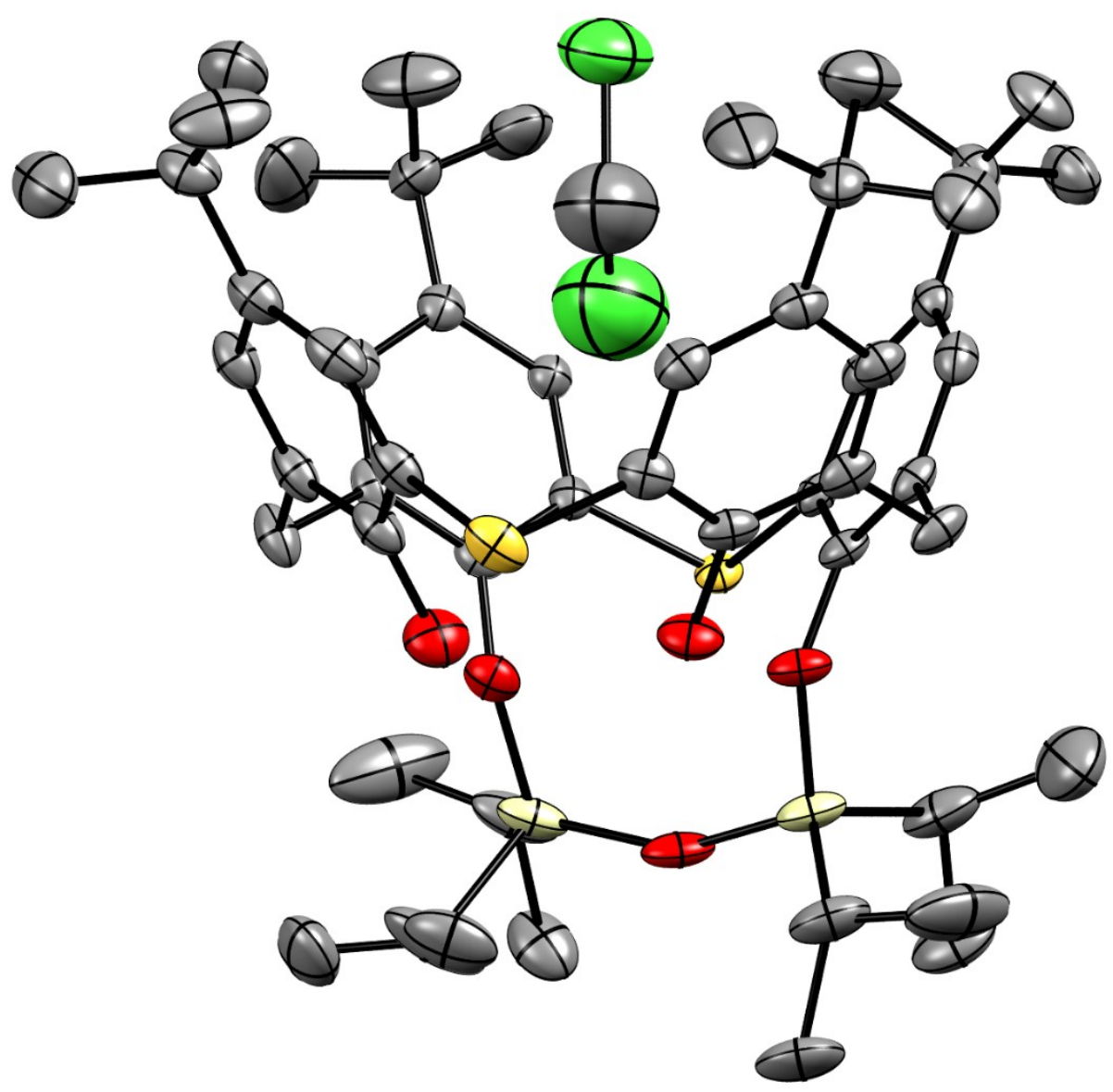

Figure S184. Thermal ellipsoid plot (50\% probability level) of compound 2. 
Crystallographic data for compound 2

$M=1054.91 \mathrm{~g} \cdot \mathrm{mol}^{-1}$, triclinic system, space group $P-1, a=11.522(8) \AA, b=11.906(6) \AA$, $c=21.106(10) \AA, \alpha=88.428(15)^{\circ}, \beta=80.74(2)^{\circ}, \gamma=84.16(3)^{\circ}, V=2843(3) \AA^{3}, Z=2$, $D_{c}=1.232 \mathrm{~g} . \mathrm{cm}^{-3}, \mu(\mathrm{Cu}-\mathrm{K} \alpha)=0.321 \mathrm{~mm}^{-1}$, crystal dimensions of $0.51 \times 0.64 \times 0.69 \mathrm{~mm}$. Data were collected on a Bruker D8 VENTURE system equipped with a multilayer monochromator and a Mo K $\alpha$ Incoatec microfocus sealed tube $(\lambda=0.71073 \AA)$ using combined $\varphi$ and $\omega$ scans at $180(2) \mathrm{K}$. The structure was solved by direct methods ${ }^{1}$ and anisotropically refined by full matrix least squares on F squared using the CRYSTALS suite of programs ${ }^{2}$ to the final values $R=0.084$ and $w R=0.222$ using 8425 independent reflections $\left(\Theta_{\max }=25.1^{\circ}\right), 656$ parameters and 52 restraints. The hydrogen atoms present in structure model were placed in calculated positions and refined with riding constraints. The disordered solvent positions were found in difference electron density maps and refined with distance, DELU and SIMU ADP restraints. The disordered functional group positions were found in difference electron density maps and refined with restrained geometry. The occupancy was constrained to full for each functional group. The MCE program ${ }^{3}$ was used for visualization of residual electron density maps. The structure was deposited into Cambridge Structural Database under number CCDC 2083300. 


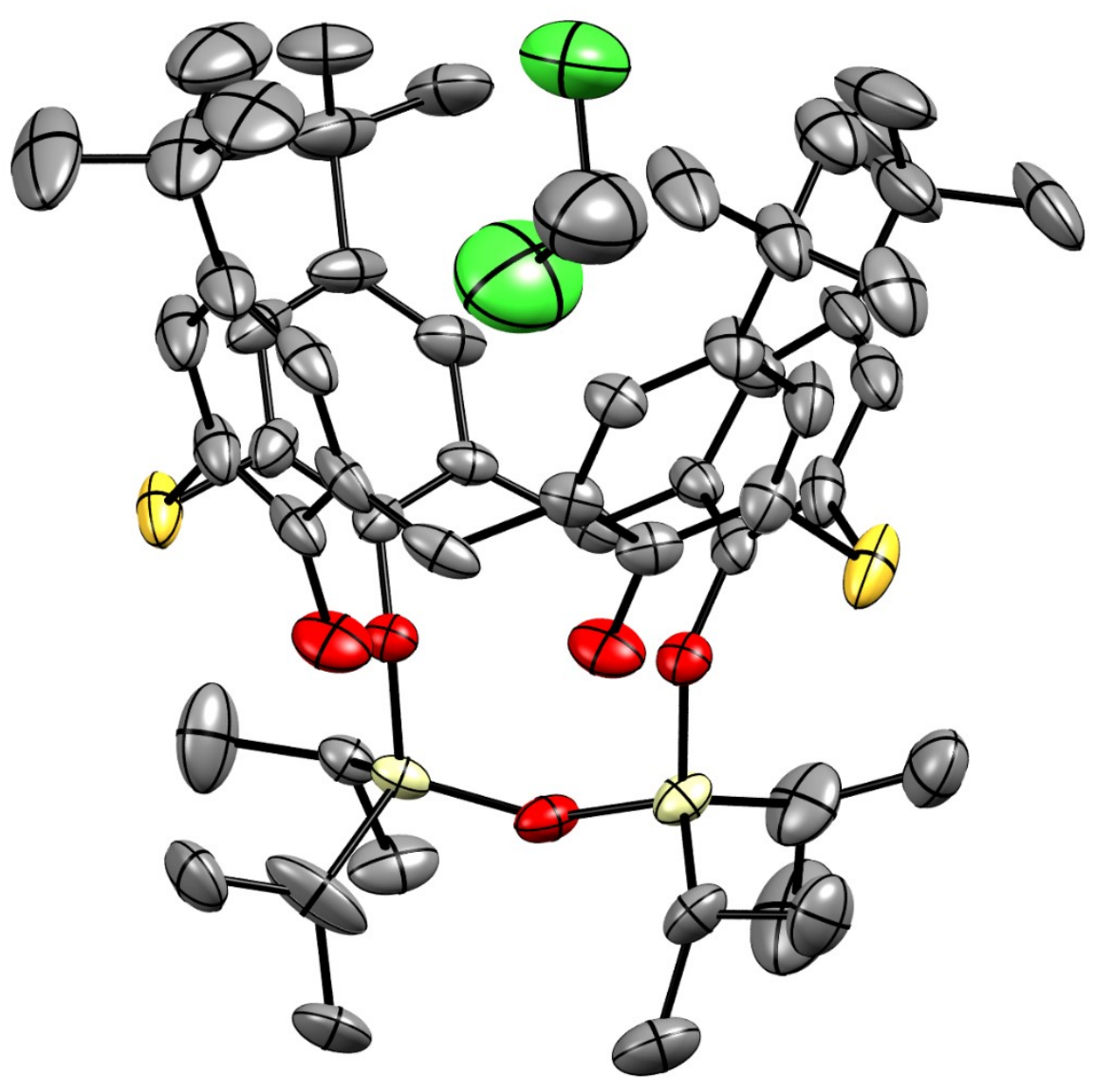

Figure S185. Thermal ellipsoid plot (50\% probability level) of compound 3. 
Crystallographic data for compound $\mathbf{3}$

$M=1033.68$ g.mol $^{-1}$, monoclinic system, space group $P 22_{1} / n, a=11.4687(6) \AA$, $b=19.4303(13) \AA, c=26.4701(16) \AA, \quad \beta=101.5411(19)^{\circ}, \quad V=5779.3(6), \quad Z=4, \AA^{3}$, $D_{c}=1.188 \mathrm{~g} . \mathrm{cm}^{-3}, \mu(\mathrm{Cu}-\mathrm{K} \alpha)=0.293 \mathrm{~mm}^{-1}$, crystal dimensions of $0.47 \times 0.61 \times 0.65 \mathrm{~mm}$. Data were collected on a Bruker D8 VENTURE system equipped with a multilayer monochromator and a Mo K $\alpha$ Incoatec microfocus sealed tube $(\lambda=0.71073 \AA)$ using combined $\varphi$ and $\omega$ scans at $180(2) \mathrm{K}$. The structure was solved by charge-flipping algorithm ${ }^{4}$ and anisotropically refined by full matrix least squares on F squared using the CRYSTALS suite of programs ${ }^{2}$ to the final values $R=0.094$ and $w R=0.205$ using 9797 independent reflections $\left(\Theta_{\max }=27.1^{\circ}\right), 774$ parameters and 271 restraints. The hydrogen atoms present in structure model were placed in calculated positions and refined with riding constraints. The disordered solvent positions and the disordered functional group positions were found in difference electron density maps and refined with distance, DELU and SIMU ADP restraints. The occupancy was constrained to full for each disordered group or solvent molecule. The MCE program ${ }^{3}$ was used for visualization of residual electron density maps. The structure was deposited into Cambridge Structural Database under number CCDC 2083302. 


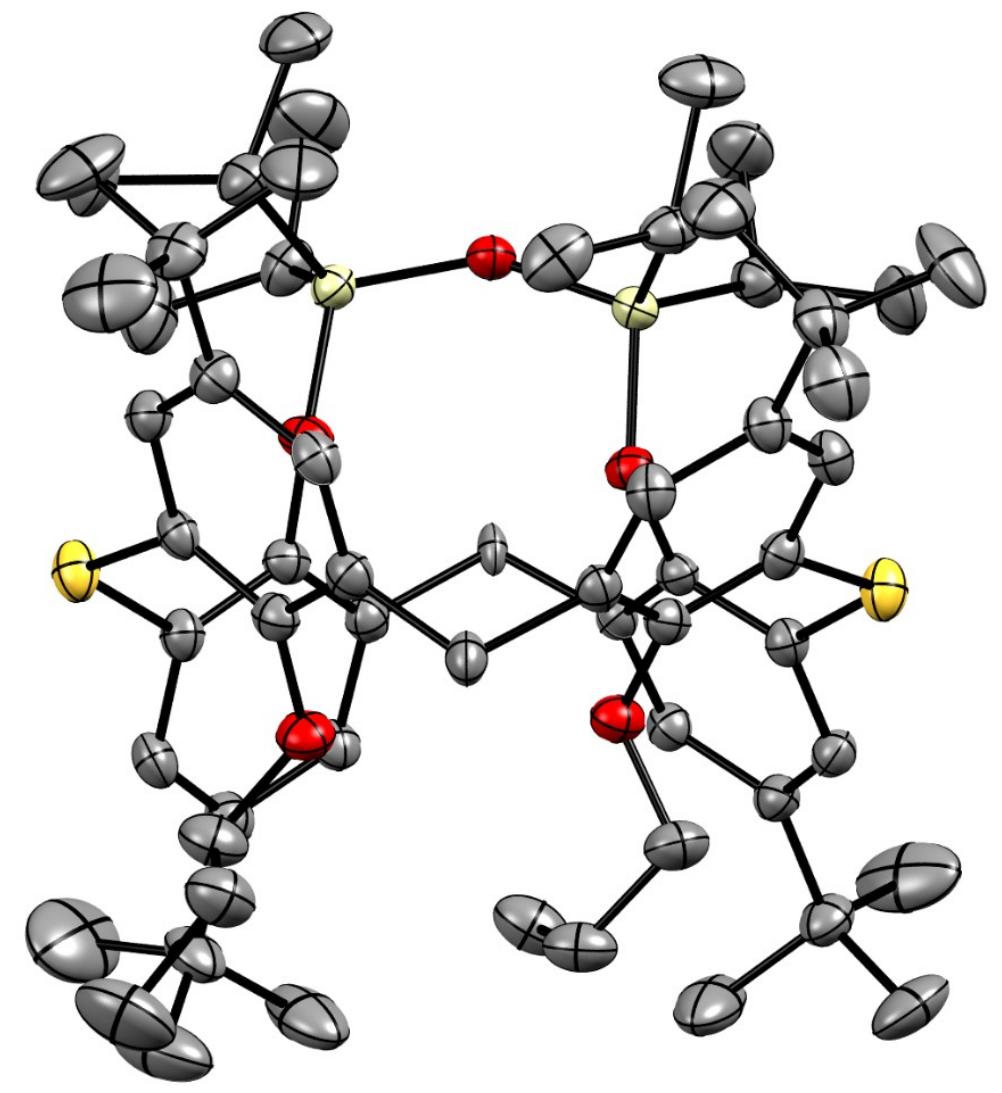

Figure S186. Thermal ellipsoid plot (50\% probability level) of compound 5 (bridge up). 


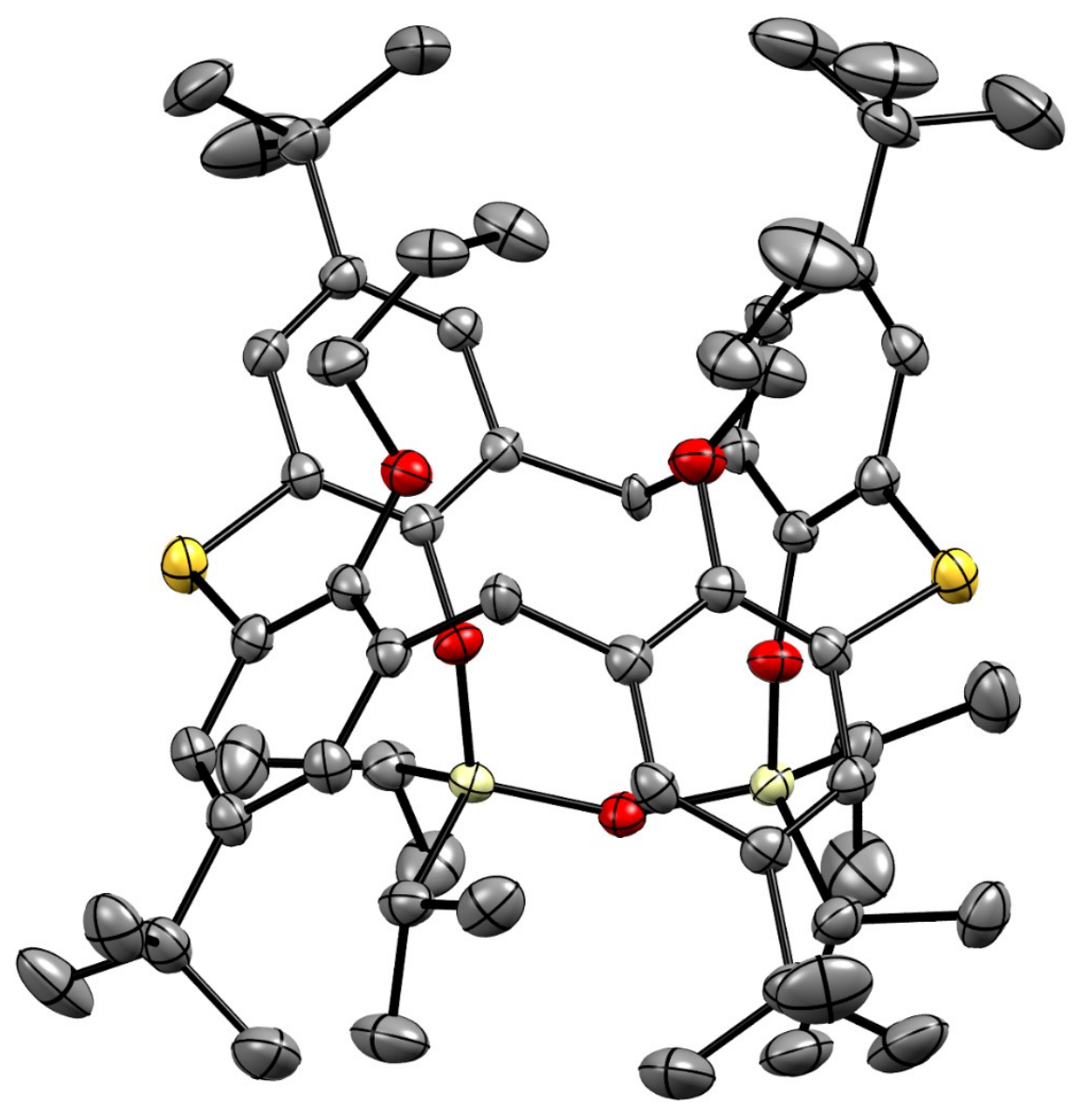

Figure S187. Thermal ellipsoid plot (50\% probability level) of compound 5 (bridge down). 
Crystallographic data for compound $\mathbf{5}$

$M=1011.67$ g.mol ${ }^{-1}$, orthorhombic system, space group $P \quad b c a, a=15.5032(4) \AA$, $b=16.8790(5) \AA, \quad c=45.3178(12) \AA, \quad V=11858.7(6) \AA^{3}, Z=8, D_{c}=1.133$ g.cm ${ }^{-3}$, $\mu(\mathrm{Cu}-\mathrm{K} \alpha)=1.542 \mathrm{~mm}^{-1}$, crystal dimensions of $0.08 \times 0.25 \times 0.42 \mathrm{~mm}$. Data were collected on a Bruker D8 VENTURE system equipped with a multilayer monochromator and a $\mathrm{Cu} \mathrm{K} \alpha$ Incoatec microfocus sealed tube $(\lambda=1.54178 \AA)$ using combined $\varphi$ and $\omega$ scans at $180(2) \mathrm{K}$. The structure was solved by direct methods ${ }^{1}$ and anisotropically refined by full matrix least squares on F squared using the CRYSTALS suite of programs ${ }^{2}$ to the final values $R=0.052$ and $w R=0.134$ using 10310 independent reflections $\left(\Theta_{\max }=70.1^{\circ}\right), 669$ parameters and 66 restraints. The hydrogen atoms present in structure model were placed in calculated positions and refined with riding constraints. The disordered functional group positions were found in difference electron density maps, refined with restrained geometry and ADP restraints. The occupancy was constrained to full for each functional group. The MCE program ${ }^{3}$ was used for visualization of residual electron density maps. The structure was deposited into Cambridge Structural Database under number CCDC 2083298. 


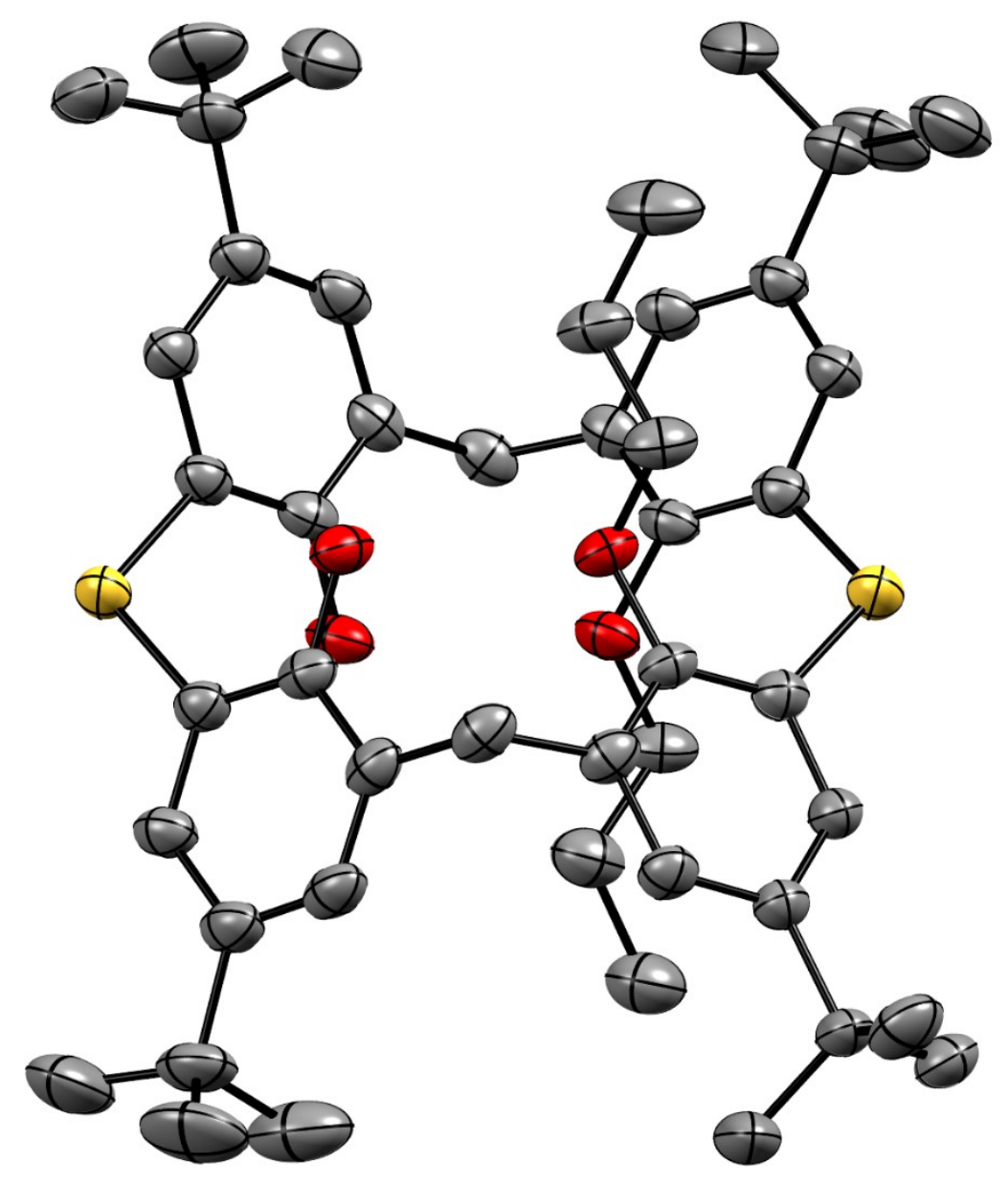

Figure S188. Thermal ellipsoid plot (50\% probability level) of compound 7. 
Crystallographic data for compound 7

$M=784.68$ g.mol ${ }^{-1}$, monoclinic system, space group $P 2{ }_{1} / c, a=15.8120(9) \AA, b=23.2912(14)$ $\AA, c=13.0474(8) \AA, V=4576.3(5) \AA^{3}, Z=4, D_{c}=1.139 \mathrm{~g} . \mathrm{cm}^{-3}, \mu(\mathrm{Cu}-\mathrm{K} \alpha)=1.373 \mathrm{~mm}^{-1}$, crystal dimensions of $0.11 \times 0.13 \times 0.68 \mathrm{~mm}$. Data were collected on a Bruker D8 VENTURE system equipped with a multilayer monochromator and a $\mathrm{Cu} \mathrm{Ka}$ Incoatec microfocus sealed tube $(\lambda=1.54178 \AA$ ) using combined $\varphi$ and $\omega$ scans at 180(2) K. The structure was solved by direct methods ${ }^{1}$ and anisotropically refined by full matrix least squares on $\mathrm{F}$ squared using the CRYSTALS suite of programs ${ }^{2}$ to the final values $R=0.074$ and $w R=0.208$ using 6053 independent reflections $\left(\Theta_{\max }=68.2^{\circ}\right), 510$ parameters and 10 restraints. The hydrogen atoms present in structure model were placed in calculated positions and refined with riding constraints. The "hetero" hydrogen atoms positions were refined with restraints. The disordered hydrogen atoms posititions were refined with riding constraints, the fractional occupancy was was fixed to 0.5. The structure was deposited into Cambridge Structural Database under number CCDC 2083297. 


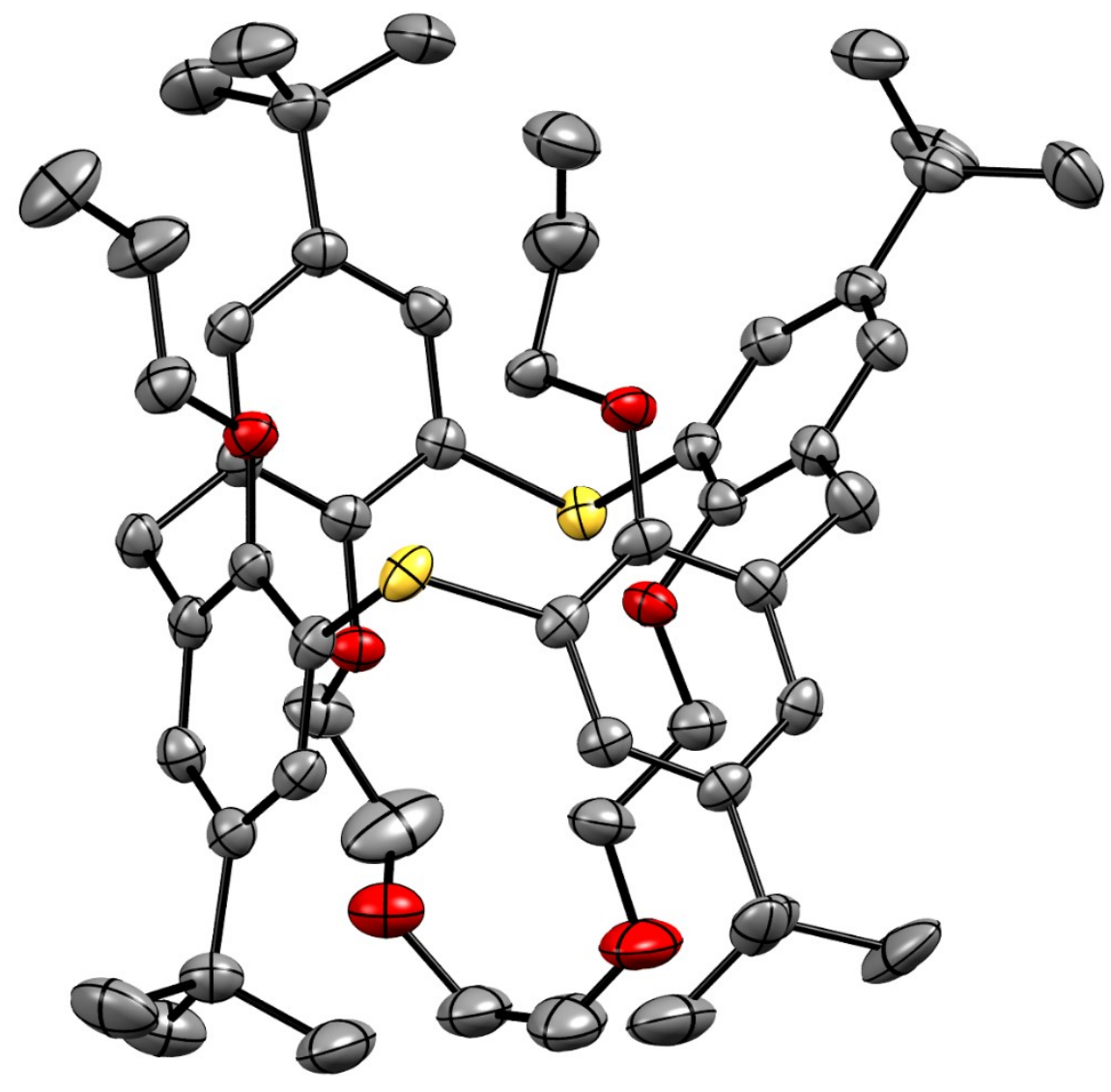

Figure S189. Thermal ellipsoid plot (50\% probability level) of compound $\mathbf{1 4 .}$ 
Crystallographic data for compound $\mathbf{1 4}$

$M=925.78 \mathrm{~g} \cdot \mathrm{mol}^{-1}$, triclinic system, space group $P-1, a=12.9951(8) \AA, b=13.3145(9) \AA$, $c=15.2018(11) \AA, \alpha=89.416(2)^{\circ}, \beta=89.714(2)^{\circ}, \gamma=79.770(2)^{\circ}, V=2588.3(3) \AA^{3}, Z=2$, $D_{c}=1.188 \mathrm{~g} . \mathrm{cm}^{-3}, \mu(\mathrm{Cu}-\mathrm{K} \alpha)=0.202 \mathrm{~mm}^{-1}$, crystal dimensions of $0.33 \times 0.34 \times 0.43 \mathrm{~mm}$. Data were collected on a Bruker D8 VENTURE system equipped with a multilayer monochromator and a Mo K $\alpha$ Incoatec microfocus sealed tube $(\lambda=0.71073 \AA)$ using combined $\varphi$ and $\omega$ scans at $180(2) \mathrm{K}$. The structure was solved by charge-flipping algorithm ${ }^{4}$ and anisotropically refined by full matrix least squares on F squared using the CRYSTALS suite of programs ${ }^{2}$ to the final values $R=0.046$ and $w R=0.111$ using 8145 independent reflections $\left(\Theta_{\max }=25.3^{\circ}\right), 671$ parameters and 62 restraints. The hydrogen atoms present in structure model were placed in calculated positions and refined with riding constraints. The disordered dichloromethane molecule was found in difference electron density maps near inversion center and refined restrained with respect to the close symmetry element. The disordered functional group positions were found in difference electron density maps and refined with restrained geometry, DELU, SIMU and ADP restraints. The occupancy was constrained to full for each functional group. The MCE program ${ }^{3}$ was used for visualization of residual electron density maps. The structure was deposited into Cambridge Structural Database under number CCDC 2083299. 


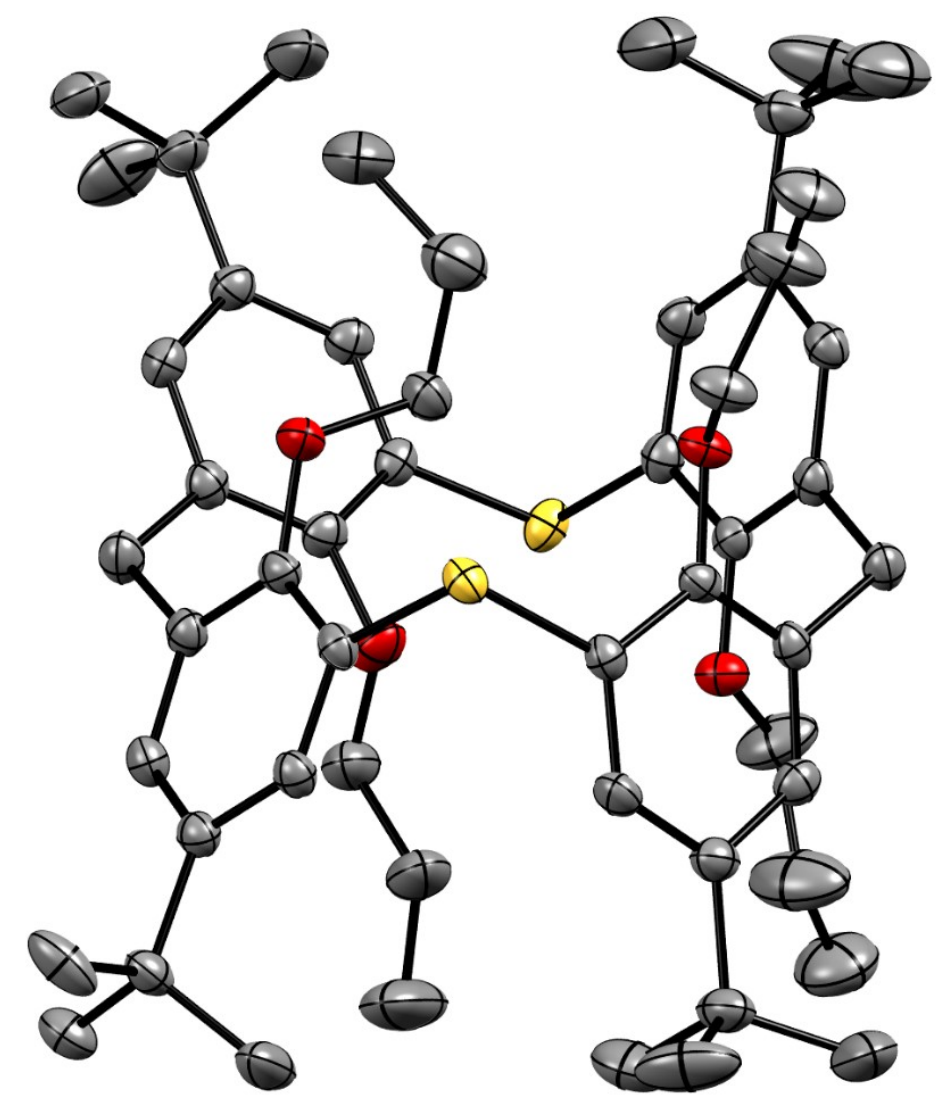

Figure S190. Thermal ellipsoid plot (50\% probability level) of compound 17. 
Crystallographic data for compound $\mathbf{1 7}$

$M=682.46 \mathrm{~g} \cdot \mathrm{mol}^{-1}$, triclinic system, space group $P-1, a=10.7681(8) \AA, b=13.0888(9) \AA$, $c=30.261(2) \AA, \alpha=96.443(2)^{\circ}, \beta=92.466(2)^{\circ}, \gamma=113.803(2)^{\circ}, V=3859.2(5) \AA^{3}, Z=4$, $D_{c}=1.175 \mathrm{~g} . \mathrm{cm}^{-3}, \mu(\mathrm{Cu}-\mathrm{K} \alpha)=0.216 \mathrm{~mm}^{-1}$, crystal dimensions of $0.28 \times 0.33 \times 0.55 \mathrm{~mm}$. Data were collected on a Bruker D8 VENTURE system equipped with a multilayer monochromator and a Mo K $\alpha$ Incoatec microfocus sealed tube $(\lambda=0.71073 \AA)$ using combined $\varphi$ and $\omega$ scans at $180(2) \mathrm{K}$. The structure was solved by charge-flipping algorithm ${ }^{4}$ and anisotropically refined by full matrix least squares on F squared using the CRYSTALS suite of programs ${ }^{3}$ to the final values $R=0.057$ and $w R=0.136$ using 11382 independent reflections $\left(\Theta_{\max }=25.4^{\circ}\right), 839$ parameters and no restraints. The hydrogen atoms present in structure model were placed in calculated positions and refined with riding constraints. The structure was deposited into Cambridge Structural Database under number CCDC 2083301.

1. Altomare, A.; Cascarano, G.; Giacovazzo, C.; Guagliardi, A.; Burla, M. C.; Polidori, G.; Camalli, M. J. Appl. Cryst. 1994, 27, 435.

2. Betteridge, P. W.; Carruthers, J. R.; Cooper, R. I.; Prout, K.; Watkin, D. J. J. Appl. Cryst. 2003, 36, 1487.

3. Rohlicek J.; Husak M. J. Appl. Cryst. 2007, 40, 600-601.

4. Palatinus L.; Chapuis G. J. Appl. Cryst. 2007, 40, 786-790. 


\section{RESULTS OF QUANTUM CHEMICAL CALCULATIONS}

\section{Compound 2}

Final single point energy: -3854.5565399858

\begin{tabular}{|c|c|}
\hline $\mathrm{C}$ & -2.82946858476447 \\
\hline C & -3.00333475191201 \\
\hline $\mathrm{C}$ & -3.28282181278284 \\
\hline $\mathrm{C}$ & -3.36877921290241 \\
\hline $\mathrm{C}$ & -3.15300991797794 \\
\hline $\mathrm{C}$ & -2.87510494064055 \\
\hline $\mathrm{C}$ & 2.84364909819198 \\
\hline C & 2.79373372532294 \\
\hline $\mathrm{C}$ & 2.93272132596839 \\
\hline $\mathrm{C}$ & 2.70088156706424 \\
\hline $\mathrm{C}$ & 2.91841704093755 \\
\hline $\mathrm{C}$ & 2.77671180138867 \\
\hline $\mathrm{C}$ & -1.27414630642023 \\
\hline C & -0.09824860330967 \\
\hline $\mathrm{C}$ & -1.29125278621985 \\
\hline $\mathrm{C}$ & 1.10240917486981 \\
\hline $\mathrm{C}$ & -0.07741795762330 \\
\hline $\mathrm{C}$ & 1.12989587555690 \\
\hline $\mathrm{C}$ & -1.21897327352799 \\
\hline C & -1.10207106468010 \\
\hline $\mathrm{C}$ & -0.06220479041261 \\
\hline C & 0.13477140004148 \\
\hline $\mathrm{C}$ & 1.19957644786810 \\
\hline C & 1.26955234244815 \\
\hline O & -2.56524083373365 \\
\hline O & -0.10076989485294 \\
\hline O & 2.81997883341601 \\
\hline O & -0.04834907674685 \\
\hline S & -2.89582223600293 \\
\hline C & -2.60790484813715 \\
\hline S & 2.73226800741674 \\
\hline C & 2.45420821844519 \\
\hline $\mathrm{C}$ & 3.01558823304644 \\
\hline C & 4.31858032833049 \\
\hline $\mathrm{C}$ & 3.02588714673665 \\
\hline C & 1.80023722482782 \\
\hline $\mathrm{C}$ & 0.20416761630790 \\
\hline C & 1.64736596564999 \\
\hline $\mathrm{C}$ & -0.36069503533028 \\
\hline C & -0.63570592139199 \\
\hline $\mathrm{C}$ & -3.68741955845363 \\
\hline $\mathrm{C}$ & -3.71532760012171 \\
\hline $\mathrm{C}$ & -2.60873672023616 \\
\hline $\mathrm{C}$ & -5.07365618456971 \\
\hline $\mathrm{C}$ & -0.07643186150073 \\
\hline C & -1.49318539908384 \\
\hline $\mathrm{C}$ & 0.61800700617326 \\
\hline $\mathrm{C}$ & 0.70792592283432 \\
\hline $\mathrm{Si}$ & 0.22463943255254 \\
\hline $\mathrm{Si}$ & 3.04469986400797 \\
\hline O & 1.69766738107524 \\
\hline $\mathrm{C}$ & 4.6 \\
\hline
\end{tabular}

$-0.58155806310543$

$-1.00773398984711$

$-0.07603790146431$

1.29436788791884

1.68176612181282

0.77856564756710

0.52726471601007

$-0.87270156483445$

1.36799334961396

$-1.38058608543109$

0.88028564945860

$-0.50374360226153$

3.41539641673426

4. 17108398915861

2.03622633287317

3.46640076096006

1.37800595204105

2.09480493044770

$-3.00759500968579$

$-3.48530672555597$

$-2.82393753103604$

$-3.80242789221073$

$-3.10511141601318$

$-3.59281827942304$

$-1.49819577593212$

0.06641671792752

$-1.76880957092089$

$-2.39609715234179$

$-2.77409391306753$

1. 27702339791491

1.29147167312158

$-2.86090211021445$

1.84952863638578

2.67664917698285

1. 11110830300834

2.80654031089085

$-4.34004555070246$

$-4.66872563230282$

$-3.28052550652213$

$-5.63545177217989$

2.29542786472332

3. 75246855327771

2.19032779983081

1. 96606575467485

5.69198378592590

6.28149690646085

6.38838060779338

6.00329200063859

$-0.77290332171591$

$-2.00574434223820$

$-1.52109130240051$

$-1.26894905864697$
0.26011672564654

$-1.07053843847467$

$-2.06883593134414$

$-1.78122405380133$

$-0.45233901348319$

0.58295892358081

0.74501071823493

0.57487298066326

$-0.36920657804896$

$-0.73828908497487$

$-1.67966148714089$

$-1.82364439333755$

1.85085147148769

1.80349665578909

2.10585398253962

1. 96840857381566

2.38870770842785

2.23398949572377

$-2.04616385048490$

$-3.35828853531788$

$-1.26230715365890$

$-3.93042202596360$

$-1.82545209351656$

$-3.13184907664987$

1.25224170459349

2. 75519531136904

1.59356271785296

0.02388186274316

$-1.41829768719142$

2. 00328115386646

2.37253883122103

$-1.00316841375694$

$-2.87186436426614$

$-2.76769379681375$

$-4.22429122912924$

$-2.85510478732330$

$-5.37271750910421$

$-5.80342689429622$

$-6.34924563759705$

$-5.48586293869984$

$-2.90821969134190$

$-2.40635222366368$

$-4.01275823703678$

$-3.51315828682035$

1. 56426296356551

1.42175590847425

2.75977453313573

0.26666020788342

4. 19609598837400

3. 24050561335272

4. 07997008071011

3. 93749103759003 
$-3.88909916953810$

$-4.82046095563818$

$-4.29984457998838$

$-0.79525307221489$

$-0.23262622235941$

$-2.06562458381723$

0.34597080197990

$-3.32487264655624$

$-2.48214248126239$

1. 74568181273945

$-0.38925038030747$

0.43038541051971

1. 94963037793065

$-3.29368155535142$

$-3.37823877421963$

$-0.43870818888427$

2.73363405679368

2.43657711316330

$-0.93933072321086$

3. 90278380205970

3. 98311482950224

$-3.61357052617639$

$-3.80969754449141$

$-2.37343562385886$

$-2.10088170439169$

3.37450609610121

2. 02363906471185

3. 26569098244981

1. 83838000915718

0.53590490389143

0.42502822430553

3.50889498118656

3. 39412955373756

2. 24410274076578

$-5.06266734843690$

$-5.42882565527511$

$-3.78073935935096$

$-3.65154123467499$

$-2.35221996110643$

$-3.03383654936697$

$-6.02786212528379$

$-5.46227161396783$

$-6.40959784833063$

4. 42566293377147

4.05636563911464

3. 90911916153933

2. 88591954656462

1.18284639454890

2.43800878783584

2.66871325481909

2.03760951042305

0.95412021941918

5.85571646313015

7.36460837187132

6.11307006757777

7.47436564427679

6.03798597281635

6.19369696055681
3. 44811159143051

2. 47951761367762

4. 91574924926614

2.84371449745473

5.06764916305020

4.25551467638596

5.74350198665601

3.42429965357677

5.66318703687025

5.63126916072026

6.96381082156114

2.69039252929096

2. 29675916061919

$-1.53800378759357$

$-0.04657712907590$

$-3.08145273235526$

$-0.19197853461665$

$-0.18620690643440$

$-2.81498604935974$

1.69344691860081

1.90039945707422

$-3.92832805943419$

$-3.52821778331620$

0.91914589647747

0.34944466693833

$-3.61144797065428$

$-2.78128114276476$

$-1.84481222452050$

$-5.04128500291446$

$-4.38357160033342$

$-4.30671811729721$

$-3.69757920955827$

$-1.93149079764087$

$-2.93501442474597$

$-6.82648084096270$

$-5.15753378997132$

$-5.79320057278976$

$-7.38149640563993$

$-6.30052358577837$

$-6.12149204438711$

$-6.51020376626212$

$-5.23587539659926$

$-4.80970069813798$

$-3.24267905332171$

$-1.98558454003776$

$-1.64369902753270$

$-4.83166294794709$

$-4.43851335067246$

$-3.61645125634726$

$-4.32058774991209$

$-2.75290764816876$

$-3.93167923950698$

0.56435584737938

1.26446999901196

2. 32153303103682

2.60496691152128

2.89250599118353

3. 69364754141597 


$\begin{array}{ll}\mathrm{H} & 0.23523845230151 \\ \mathrm{H} & 1.74216387195696 \\ \mathrm{H} & 0.73804226629385 \\ \mathrm{H} & 5.13233346635190 \\ \mathrm{H} & 4.35172978591632 \\ \mathrm{H} & 1.44444539385356 \\ \mathrm{H} & 2.71281891934160 \\ \mathrm{H} & 2.86257583088786 \\ \mathrm{H} & 1.96316678586966 \\ \mathrm{H} & 3.58991531304389 \\ \mathrm{H} & 3.31398138470404 \\ \mathrm{H} & 5.30382379625534 \\ \mathrm{H} & 5.80505522391968 \\ \mathrm{H} & 6.63564705144814 \\ \mathrm{H} & 4.09541047405695 \\ \mathrm{H} & 5.53905821752747 \\ \mathrm{H} & 3.93762133805997 \\ \mathrm{H} & -2.01891144346905 \\ \mathrm{H} & -0.88959131685114 \\ \mathrm{H} & -0.12404136581833 \\ \mathrm{H} & -0.49219365315361 \\ \mathrm{H} & -1.77763366124034 \\ \mathrm{H} & -0.87751906995037 \\ \mathrm{H} & -2.52564683252451 \\ \mathrm{H} & -1.94936370401091 \\ \mathrm{H} & 1.88683062594906 \\ \mathrm{H} & 0.74898061378593 \\ \mathrm{H} & 0.33393962591944 \\ \mathrm{H} & 1.86159449771178 \\ \mathrm{H} & 0.32664075903315 \\ \mathrm{H} & 0.65607131525714\end{array}$
5.52766540407852
5.64719185822033
7.08554175622233
$-2.16130875637782$
$-4.03398601699196$
$-4.66362426568852$
$-5.87286126515575$
$-4.67692448267338$
$-4.20554312587841$
$-3.68675739321393$
$-5.34610943983124$
0.11770020622600
$-1.55163005674024$
$-0.57099767707816$
0.69890187423601
0.01222756523392
$-0.60960373070324$
$-1.55106366886021$
0.49222992022989
$-3.94528958650421$
$-3.09467126632480$
$-3.94028631344069$
$-3.03724100872896$
$-3.14696778178649$
$-1.62788300246454$
1.68356547835035
2. 26709939566298
2.37071103825217
$-0.55152539560392$
$-1.36385299532339$
0.21588929735784

$-0.60071319305227$

0.32128765224234

0.08697401675090

4. 39286847856361

3. 25709337737234

2. 49871232795033

2. 74024884093316

1.44823352012156

5.17791679515073

5.62776034240827

5.08182578753935

2. 35043461194356

2.06416371304184

3. 28157696711935

4.71710730355068

5.47247955395119

5.89649972906944

3.76749642418165

5.94257037903650

3.92767632929406

2. 42528603072048

3.30934647594719

6.20525152835475

5.58631231497519

6.28275121406113

5.37821725919706

6.59634901926067

4.87948911525509

6.82869955540848

7.15130236950266

7.87180040813720 


\section{Compound $\mathbf{3}$}

\section{Final single point energy: -3854.54320150886}

C $\quad-2.79457267514222$

C $\quad-2.87555889457051$

C $\quad-3.17330443686201$

C $\quad-3.37642360515619$

C $\quad-3.27055499725087$

C $\quad-2.97115803664623$

C 2.73322453951045

C 2.75531783391637

C 2.87884504952226

C 2.82822435579639

C 3.01116399813168

C 2.97109389288758

C $\quad-1.26417535010331$

C $\quad-0.08006722113167$

C $\quad-1.26289102836122$

C 1.11900044580426

C $\quad-0.04470112246313$

C 1.16386936940590

C $\quad-1.23980449846435$

C $\quad-1.14811984368635$

C $\quad-0.05792147120694$

C $\quad 0.06186292432437$

C 1.17669579886848

C 1.21839503868919

O -2.43985252044538

O $\quad-0.02191226419746$

o 2.68516745859283

O $\quad-0.02660251397218$

C $\quad-2.61515161560316$

S $\quad-2.84166841464929$

C 2.48662479142665

S $\quad 2.71052462224896$

C $\quad 3.16220922579089$

C $\quad 4.42304876673312$

C 3.29899417388443

C 1.91515878228758

C 0.08218705087114

C $\quad 1.49435738160047$

C $\quad-0.40305493283934$

C $\quad-0.85320402143282$

C $\quad-3.69838087374966$

C $\quad-3.87741896629689$

C $\quad-2.54077512151818$

C $\quad-5.01044021152187$

C $\quad-0.05112676373220$

C $\quad-1.46612486770462$

C 0.72265347212895

C 0.65721211428798

Si 0.19791566282681

Si 2.97866593707628

O 1.68636169217157

C $\quad 4.62514920670330$

C $\quad 3.20214815476517$

C 2.32416955281391

C $\quad 3.13413279087312$
$-0.75605686065861$

$-1.19517807080737$

$-0.25507284638178$

1.10441377779971

1.50130591413339

0.59318647135522

0.60355660562711

$-0.80064745108941$

1.41100951202372

$-1.32675123400502$

0.91072353169912

$-0.47886674580563$

3.41325884131301

4.13992324251101

2.04090321893865

3. 41462204735961

1.36484522009577

2.04983986795553

$-2.94014737670923$

$-3.33609833726068$

$-2.86411335681975$

$-3.66905071276542$

$-3.16687090684440$

$-3.56620604152013$

$-1.65391443142229$

0.05970822905960

$-1.67506633677683$

$-2.52582357759668$

$-2.65150488775710$

1.17420541994138

1.30078598188490

$-3.09783809529600$

1.86976769659615

2.74525585783409

1.11713451611230

2.78218372291657

$-4.09893197519507$

$-4.50277045324863$

$-2.92491288113711$

$-5.31433194536819$

2.08858583143689

3. 53273907218379

2. 07846824387392

1.65814172293427

5.65407737311779

6.26055720641536

6.36902068493874

5.92363693549088

$-0.73399387433140$

$-2.01348040903852$

$-1.47671797329163$

$-1.26452827675625$

$-3.89441160999561$

$-4.77851546498085$

$-4.36535940136850$
0.25754429535960

$-1.07461941732465$

$-2.06239723424298$

$-1.77811890934112$

$-0.44037974031703$

0.58445450426997

0.81779280496248

0.66206715334493

$-0.31501541298715$

$-0.64638941159736$

$-1.61499836489035$

$-1.74917036410349$

1. 91582674657105

1.76925940760192

2.21175341384190

1.87456928209035

2.41501895120727

2.16197956283182

$-2.04501472563338$

$-3.38324039687951$

$-1.27521267796064$

$-4.00884677526665$

$-1.88736587168343$

$-3.22777179030239$

1.25922923946879

2. 79549444715196

1.69364448438340

0.03516349482100

$-1.44960822378329$

2.29139144002690

2.15365434748851

$-0.95432781556652$

$-2.80991114179682$

$-2.61757136050696$

$-4.14736861122012$

$-2.89709033583347$

$-5.48788020158288$

$-5.95640119935553$

$-6.37181523994852$

$-5.69512171664203$

$-2.91862077458460$

$-2.41088052393754$

$-3.94567172063655$

$-3.61834430792086$

1. 49068483990357

1. 41882276722417

2. 62494911948469

0.14102452214723

4. 27759301593351

3. 30125662776443

4. 20507634313066

3. 93499471515000

3. 43905652249727

2. 53624389768420

4. 90790240005840 
$-1.02194374526732$

$-0.05162556259202$

$-1.98471860917565$

0.44226204208824

$-3.29043555954793$

$-2.30903049730433$

1.81534666773832

$-0.26708486176163$

$-0.60361171341723$

2.53718809573912

2.48734060842159

$-0.94160602330323$

3.89809096355689

3. 92924500604648

$-3.39190515669530$

$-3.78941363916395$

$-2.42272150365790$

$-2.24386164337555$

$-3.30532966292606$

$-2.95751389129075$

2. 01500564892429

0.58091020719681

3. 44679200179094

2. 12533776003448

3. 33330212020378

1.83695642965069

0.49847075238563

0.46886983466373

3.47239210205072

3. 38308513348631

2. 18479912100511

$-4.82345908750978$

$-5.33572456618892$

$-3.66802360213626$

$-3.21187095484775$

$-2.05125741592729$

$-2.61714036183015$

$-5.62818466359183$

$-5.08384034171651$

$-6.16491290759539$

4.19573362176487

3. 90878192224171

3. 61641105031263

2.77006190098998

1.08420047738323

2. 38879645061465

2. 34786684820580

1. 66324522051289

0.65096022209072

5.81671649330617

7.33742460173355

6.12794929918384

7.45118215900675

6.01364902558816

6.19867082545184

5.43356093926773

5.55545989980308

7.00067079173683
2.78794253799890

4.87789141957181

4.35321394819603

5.78117396094367

3.59481179933870

5.76307640206694

5.61284431890026

7.02623667257808

$-3.09064523367324$

$-0.16150474020029$

$-0.16082785806339$

$-2.72657700741578$

1.80883265190659

1.73666323185763

$-3.95156354697168$

$-3.64904072805004$

1.23046877146226

0.39208962499959

$-0.58118913638932$

$-2.18488572618753$

2.34589035289212

2.96965267712964

$-3.45433031850066$

$-2.56756676876975$

$-1.69481001028891$

$-4.96825461136376$

$-4.35925771140356$

$-4.16144533434773$

$-3.74623034888907$

$-1.99023631493639$

$-3.03465029232235$

$-7.00401908477679$

$-5.36655685170929$

$-5.89151992597383$

$-7.43127639350741$

$-6.25380420119309$

$-6.11253318920080$

$-6.74676175405328$

$-5.42706604873642$

$-5.08382146176678$

$-3.25576925531844$

$-1.92317961074570$

$-1.70081594772709$

$-4.76975278238107$

$-4.37777601705224$

$-3.47798064903996$

$-4.43740184811700$

$-2.91376976571418$

$-4.04322615457027$

0.61019220159789

1. 22489740253594

2.35884135519477

2.44486738960266

2. 69947239799557

3. 59514970534285

$-0.68410514175246$

0.14173337635615

$-0.06663471668246$ 


$\begin{array}{ll}\mathrm{H} & 5.03217869246319 \\ \mathrm{H} & 4.24489970398689 \\ \mathrm{H} & 1.27997727726686 \\ \mathrm{H} & 2.67242661466483 \\ \mathrm{H} & 2.34620147490854 \\ \mathrm{H} & 2.12200107622212 \\ \mathrm{H} & 3.81648357809483 \\ \mathrm{H} & 3.39760601761814 \\ \mathrm{H} & 5.30671999312313 \\ \mathrm{H} & 5.76182762116399 \\ \mathrm{H} & 6.61587550436383 \\ \mathrm{H} & 4.22139743015208 \\ \mathrm{H} & 5.54190186944936 \\ \mathrm{H} & 3.86413402806423 \\ \mathrm{H} & -2.02875547409136 \\ \mathrm{H} & -0.89105020009159 \\ \mathrm{H} & -0.21317481452344 \\ \mathrm{H} & -0.54077044559037 \\ \mathrm{H} & -1.85562769987776 \\ \mathrm{H} & -1.03538447765213 \\ \mathrm{H} & -2.66544285934447 \\ \mathrm{H} & -2.05132628548710 \\ \mathrm{H} & 1.91987983800911 \\ \mathrm{H} & 0.81232815075547 \\ \mathrm{H} & 0.38464390902054 \\ \mathrm{H} & 1.82874063845962 \\ \mathrm{H} & 0.26989726804583 \\ \mathrm{H} & 0.65036659942380 \\ & \end{array}$

$-2.09446317427965$

$-4.03065504759711$

$-4.77490151401422$

$-5.82053156805821$

$-4.44497319378045$

$-4.24824836735085$

$-3.81256515890182$

$-5.42899660151423$

$-0.20536329800051$

$-1.91035163930003$

$-0.75314935038564$

0.85443374377441

0.16668796279218

$-0.22255474533122$

$-1.45372867675014$

0.62947501770300

$-3.90027975328029$

$-3.11823722420188$

$-3.89478521211448$

$-2.86422661077844$

$-2.94184930690590$

$-1.41168779698409$

1. 71165179351221

2. 37312189038530

2.42843699451175

$-0.46915346521309$

$-1.22033120562344$

0.37263002301158
4.53543959993124

3. 10152987480719

2.86325289342289

2. 57436881437676

1. 49576282027718

5.31449197712361

5.56578205368800

4. 98441201197728

2. 13100892840312

2. 15803075817918

3. 18844427182460

4.35763799282501

5.29998533316832

5.71343359078901

3.80996672716460

5.97418214751998

4.14087815702182

2. 59125199129220

3.49742396331933

6.36102160788308

5.69126632481658

6.32524522906946

5.35465302922461

6.55882751312807

4.84281842086135

6.89509937854431

7. 24765227837900

7. 91301251975195 